\title{
WestVirginiaUniversity
}

THE RESEARCH REPOSITORY @ WVU

Graduate Theses, Dissertations, and Problem Reports

2007

\section{Three essays on corporate liquidity, financial distress and equity returns}

Fang Wang

West Virginia University

Follow this and additional works at: https://researchrepository.wvu.edu/etd

\section{Recommended Citation}

Wang, Fang, "Three essays on corporate liquidity, financial distress and equity returns" (2007). Graduate Theses, Dissertations, and Problem Reports. 2599.

https://researchrepository.wvu.edu/etd/2599

This Dissertation is protected by copyright and/or related rights. It has been brought to you by the The Research Repository @ WVU with permission from the rights-holder(s). You are free to use this Dissertation in any way that is permitted by the copyright and related rights legislation that applies to your use. For other uses you must obtain permission from the rights-holder(s) directly, unless additional rights are indicated by a Creative Commons license in the record and/ or on the work itself. This Dissertation has been accepted for inclusion in WVU Graduate Theses, Dissertations, and Problem Reports collection by an authorized administrator of The Research Repository @ WVU.

For more information, please contact researchrepository@mail.wvu.edu. 


\title{
Three Essays on Corporate Liquidity, Financial Distress and Equity Returns
}

\author{
Fang Wang \\ Dissertation submitted to the \\ College of Business and Economics \\ At West Virginia University \\ in partial fulfillment of the requirements \\ for the degree of \\ Doctor of Philosophy \\ in \\ Economics \\ Ronald J. Balvers, Ph.D., Chair \\ Ashok B. Abbott, Ph.D. \\ Arabinda Basistha, Ph.D. \\ Alexei V. Egorov, Ph.D. \\ Alexander Kurov, Ph.D. \\ Department of Economics \\ Division of Economics and Finance
}

Morgantown, West Virginia
2007

Keywords: Corporate Liquidity, Equity Returns, Residual Cash, Financial Distress

C 2007 Fang Wang 


\title{
AbStract \\ Three Essays on Corporate Liquidity, Financial Distress and Equity Returns
}

\author{
Fang Wang
}

The three essays in this dissertation study the impact of corporate liquidity on a firm's value, how corporate liquidity affects the probability of financial distress and equity returns, and whether corporate liquidity represents an idiosyncratic risk, or serves as a priced risk factor.

The first essay examines the relationship between corporate liquidity and equity returns for individual firms. It is found that firms holding more liquid assets have significantly higher equity returns. This positive relationship between corporate liquidity and equity returns is more pronounced among firms with financial constraints. In addition, firms expecting volatile cash flows stock more cash for precautionary purposes and their stock returns are higher relative to those generating stable cash flows. These empirical evidences indicate that the level of corporate liquidity may signal risks faced by companies. Furthermore, more liquid firms tend to have higher market beta, which suggests that corporate liquidity may serve as a risk factor. Finally, Fama-MacBeth two pass regression results show that corporate liquidity contains different information than what is included in other important firm characteristics like size and book-to-market equity ratio.

The second essay further explores the relationship between corporate liquidity and the expected return of stocks at the aggregate level. Asset pricing tests are performed to examine whether the constructed corporate liquidity factor, LMI (Liquid Minus Illiquid), is a risk factor and whether it is systematic and priced. Empirical evidences from multifactor asset pricing models suggest that $L M I$ can still serve as an independent factor and is priced although it shares some common information with the three popular factors $M K T, S M B$ and HML. Moreover, corporate liquidity beta is increased when the macroeconomic condition becomes less favorable, and the corporate liquidity beta is 
more sensitive to changes in macroeconomic conditions for firms with more corporate liquidity and financial constraints.

The third essay examines how a company's market value can be affected by residual cash, and whether corporate liquidity and negative residual cash can predict bankruptcy. It is shown that deviation from the target or optimal cash is value destructive, which provides support for the tradeoff theory. However, the impact of residual cash on market value of equity is not symmetric: positive residual cash tends to reduce firm value less than negative residual cash. In general, firms with negative residual cash are more likely to experience financial distress since they are smaller, less profitable, generate lower cash flows, and have higher leverage but weaker payoff ability. Furthermore, logistic regression results show that negative residual cash serves as an important predictor variable for bankruptcy. The probability of filing bankruptcy is higher for firms with negative residual cash. Finally, negative residual cash contributes to different default probabilities (Altman's Z-score, Vassalou and Xing's default likelihood indicator and Chava and Jarrow's default probability) estimated in previous literature. 
Dedicated to Qiang 


\section{ACKNOWLEDGEMENTS}

I am deeply indebted to my advisor and dissertation chair, Professor Ronald Balvers, for his constant guidance, patience, and willingness to help me achieve this objective. He was always there to listen and discuss, prompt to read and revise my paper, and gave invaluable suggestions and advices that clarified my thoughts on this work. His critical and insightful way of thinking undoubtedly will benefit my future career.

I would like to express my sincere appreciation to my other committee members for their valuable time reading my dissertation and for their inspiring advices. I am grateful to Professor Alexei Egorov for his staunch support and unending encouragement, and for the countless hours he has spent helping me with scientific programming. I appreciate all the help, advice, and information I have received from Professor Ashok Abbott. I am thankful to Professor Alex Kurov for his constructive comments, suggestions and concern. My gratitude also goes to Professor Arabinda Basistha for his assistance and advice on the technical issues in econometrics. In addition to my committee, I would like to thank all the staff and faculty in my department, especially Professor Stratford Douglas for his great help with SAS programming.

During my four years in beautiful Morgantown, I was surrounded by many smart and wonderful colleagues. My gratitude is extended to Nathan Ashby, Saurav Roychoudhury, Jawad Salimi and $\mathrm{Ou} \mathrm{Hu}$ for being great officemates and friends. Their friendship and help made my time at West Virginia University truly enjoyable.

With sincere gratitude I include my parents, Changchuan and Shuping, for giving me life, for their unconditional love and support during every stage of my life and for always believing in me. They have taught me many important things in life which have been most essential for my achievements.

I give my heartfelt thanks to my precious little son, Andre, for filling my days with sweet smiles and hugs, and being a great cheering section for me. The past two years of my Ph.D. study have been delighted with every little achievement and progress he has made through his first two years of life.

Most importantly, I wish to dedicate this work to my wonderful husband, Qiang, who has been nothing but supportive, encouraging, and understanding throughout my career. He has been commuting between Pittsburgh and Morgantown every week, dedicated both to our family and his own Ph.D. study. He has spent endless hours taking care of and playing with our son, when I was buried in papers. I could not have completed this journey without his unwavering support, enthusiasm and encouragement. This dissertation is as much a tribute to him - if not more - than to me for his love and understanding during these past five years. 


\section{TABLE OF CONTENTS}

NOTICE OF COPYRIGHT.................................................................... i

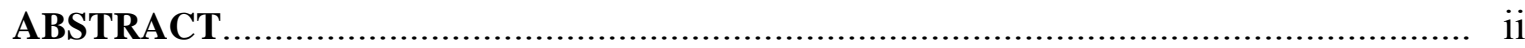

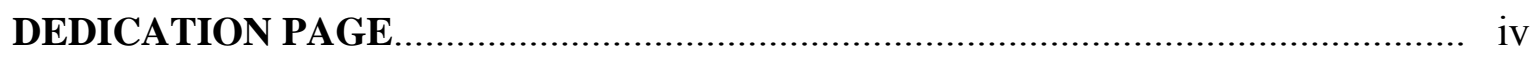

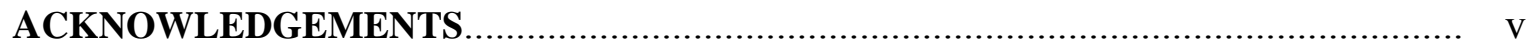

TABLE OF CONTENTS............................................................................ vi

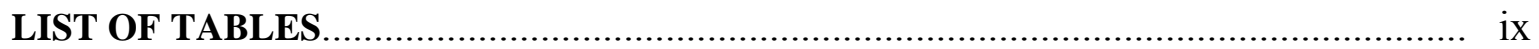

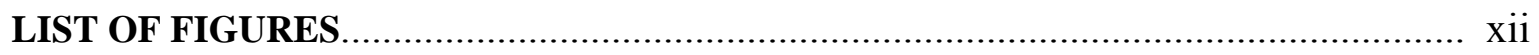

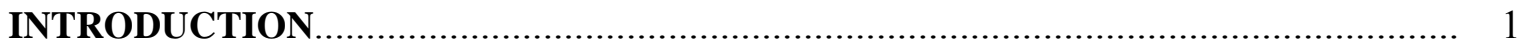

CHAPTER 1: Idiosyncratic Corporate Liquidity and Equity Returns.......................... 7

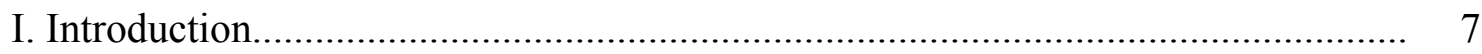

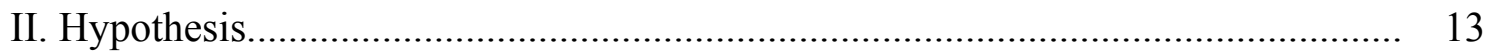

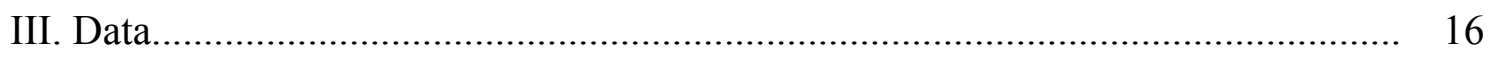

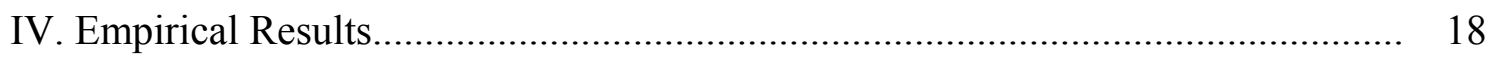

IV.1. Firm Characteristics and Corporate Liquidity...................................... 18

IV.2. The Corporate Liquidity Effect............................................................ 22

IV.2.1 The Corporate Liquidity Effect Controlled by Size....................... 22

IV.2.2 The Corporate Liquidity Effect Controlled by Book-to-Market

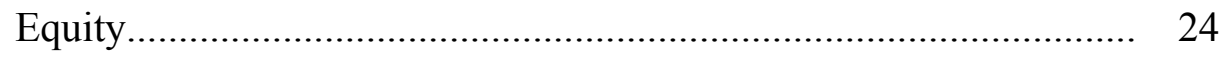

IV.3. Fama-MacBeth Two-Pass Regression................................................... 26

V. Summary and Conclusion......................................................................... 31

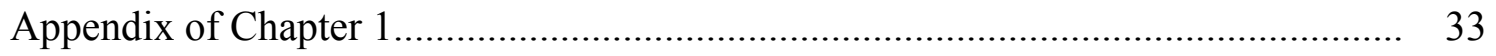

A.1. The Size Effect Controlled by Corporate Liquidity.................................. 33

A.2. The Value Effect Controlled by Corporate Liquidity.................................. 34

A.3. Additional Fama-MacBeth Two-Pass Regression..................................... 35

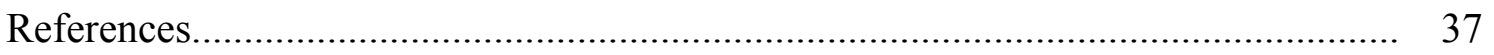


CHAPTER 2: Is Corporate Liquidity A Risk Factor? .......................................... 66

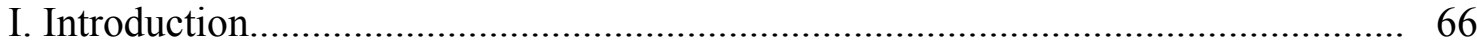

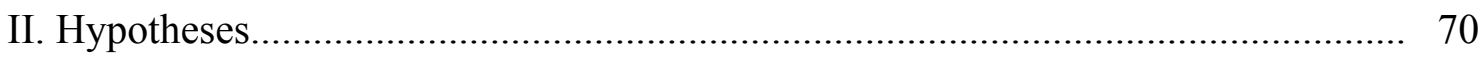

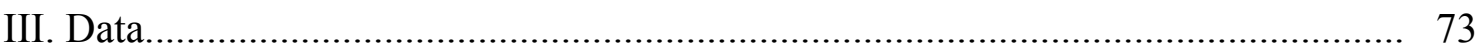

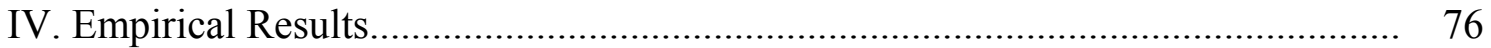

IV.1. Does the Corporate Liquidity Factor Have a Risk Premium? ................... 76

IV.2. Does Corporate Liquidity Contain the Same Information

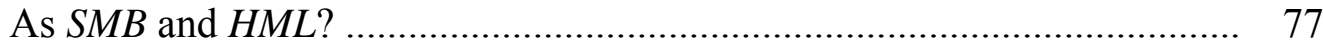

IV.3. Multifactor Asset Pricing Models on 25 Size-BE/ME portfolios.............. 79

IV.3.1 The Fama-French Three-Factor Model..................................... 80

IV.3.2 The Four-Factor Model............................................................ 82

IV.4. Multifactor Asset Pricing Models on 27 Size-BE/ME-Corporate Liquidity

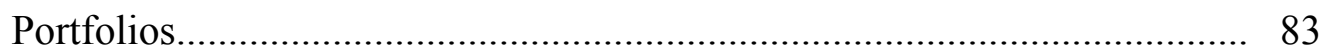

IV.4.1 The Fama-French Three-Factor Model....................................... 84

IV.4.2 The Four-Factor Model............................................................... 84

IV.5. Asset Pricing Models on 10 Corporate Liquidity Portfolios...................... 87

IV.6. Multifactor Asset Pricing Models on 25 Subsequently Sorted Size- or BE/ME-Corporate Liquidity Portfolios................................................. 88

V. Corporate Liquidity and Macroeconomic Variables...................................... 89

V. 1. Is Corporate Liquidity Factor a Proxy for Systematic Risk? .................... 89

V.2. Are Liquid Firms' Fundamentals More Cyclical? .................................. 90

V.3. Corporate Liquidity Beta and Changes in Macroeconomic Condition....... 91

V.4. Do Financial Constraints Affect the Relationship Between Corporate Liquidity Betas and Changes in Macroeconomic Variables?...... 93

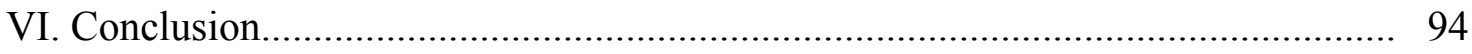

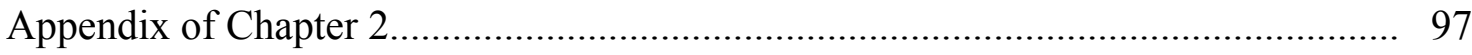

A.1. The Four-Factor Model with Decomposed Corporate Liquidity Factors..... 97

A.2. The Five-Factor Model with Decomposed Corporate Liquidity Factors..... 99

B. Future Equity Return Forecast Using Corporate Liquidity................. 99

References............................................................. 102 
CHAPTER 3: Residual Cash, Firm Value and Financial Distress.................. 142

I. Introduction.......................................................... 142

II. Hypotheses...................................................... 144

III. Data............................................................. 147

IV. Estimation of Residual Cash............................................ 149

V. Empirical Results................................................ 152

V.1. The Impact of Residual Cash on Firm Value.......................... 154

V.2. Negative Residual Cash and the Probability of Bankruptcy.............. 156

V.3. Negative Residual Cash and Financial Distress........................ 159

V.4. Forecasting Regression of Residual cash............................ 160

VI. Conclusion.......................................................... 162

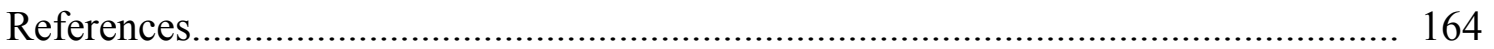




\section{LIST OF TABLES}

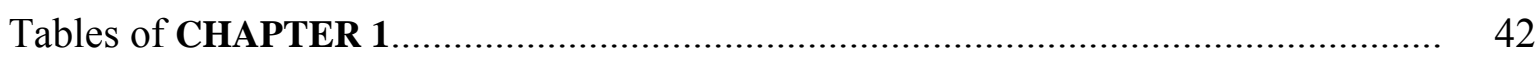

Table 1. Summary Statistics for the 1962.1 - 2005.12 Sample................................. 42

Table 2. Firm Characteristics and Corporate Liquidity............................................. 43

Table 3. Quantile Average Sorted by Firm Characteristics........................................ 45

Table 4. Corporate Liquidity Effect Controlled by Size................................... 46

Table 5. Corporate Liquidity Effect Controlled by BE/ME......................... 49

Table 6. Regression of Individual Firm Beta on Firm Characteristics............... 52

Table 7. Fama-MacBeth Second-pass Regression on Individual Firms............... 53

Table 8. Distribution of Firms across Industries and Corporate Liquidity/Corporate Liquidity Volatility Portfolios............................................... 55

Tables of Appendix of CHAPTER 1................................................ 57

Table A1. Size Effect Controlled by Corporate Liquidity......................... 57

Table A2. Value Effect Controlled by Corporate Liquidity........................ 60

Table A3. Fama-MacBeth Second-pass Regression on Individual Firms.............. 63

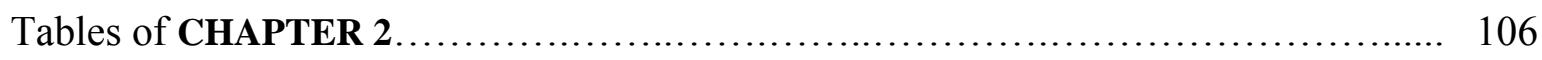

Table 1. Summary Statistics for the 1962.1 - 2005.12 Sample..................... 106

Table 2. Pricing Tests on the Corporate Liquidity Factor.......................... 107

Table 3a. The Fama-French Three-Factor Model on 25 Size-BE/ME Portfolios..... 108

Table 3b. The Market Factor + Corporate Liquidity Factor on 25 Size-BE/ME

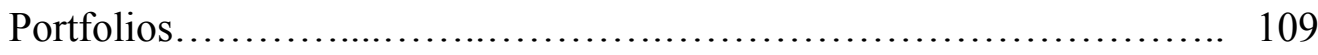

Table 4. The Fama-French Three-Factor + Corporate Liquidity Factor on 25 Size-BE/ME Portfolios.......................................... 110

Table 5. Summary Statistics on the 27 Size, BE/ME and Corporate Liquidity Sorted Portfolios

Table 6. The Fama-French Three-Factor Model on 27 Size-BE/ME-Corporate Liquidity Portfolios......................... 113

Table 7. The Fama-French Three-Factor + Corporate Liquidity Factor on 27 Size-BE/ME-Corporate Liquidity Portfolios. 
Table 8. CAPM and Multifactor Models on 10 Corporate Liquidity Portfolios.........

Table 9. The Fama-French Three-Factor Model on 25 Size -Corporate Liquidity Portfolios

Table 10. The Fama-French Three-Factor Model on 25 BE/ME-Corporate Liquidity Portfolios

Table 11. The Fama-French Three-Factor + Corporate Liquidity Factor on 25 Size-Corporate Liquidity Portfolios

Table 12. The Fama-French Three-Factor + Corporate Liquidity Factor on $25 \mathrm{BE} / \mathrm{ME}-\mathrm{Corporate}$ Liquidity Portfolios

Table 13. Time Series Regression of Corporate Liquidity Factor on Changes in Macroeconomic Variables

Table 14. Performance on Macroeconomic Changes for Liquid and Illiquid Firms... 128

Table 15. Time Series Regression of Corporate Liquidity Beta on Changes in Macroeconomic Conditions for Liquid and Illiquid Firms

Table 16. Time Series Regression of Corporate Liquidity Beta on Changes in Macroeconomic Conditions for Constrained and Unconstrained Firms.....

\section{Tables of Appendix of CHAPTER 2}

Table A1. The Fama-French Three-Factor + Corporate Liquidity Factor $\left(L M I_{\text {med_low }}\right)$ on 27 Size-BE/ME-Corporate Liquidity Portfolios

Table A2. The Fama-French Three-Factor + Corporate Liquidity Factor $\left(L M I_{\text {high_med }}\right)$ on 27 Size-BE/ME-Corporate Liquidity Portfolios

Table A3. The Fama-French Three-Factor + Corporate liquidity Factors $\left(L M I_{\text {med_low }}\right.$ and $L M I_{\text {high_med }}$ ) on 27 Size-BE/ME-Corporate Liquidity Portfolios....... 138

Table B. Returns on Zero-investment Portfolios Sorted by Size and BE/ME

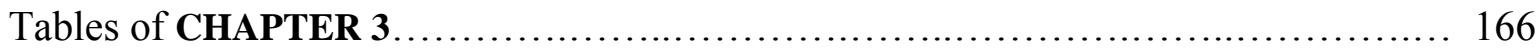

Table 1. Predicting the Normal Level of Cash..................................... 166

Table 2. Summary Statistics for Firms with Positive and Negative

Residual Cash Holdings............................................... 167

Table 3. The Impact of Normal and Residual Cash on Firm Value.................... 171

Table 4. Bankruptcies and Failures as Percentage of Active Firms................... 172 
Table 5. Logistic Regression of on Firm Characteristics as Bankruptcy Predictors..... 173

Table 6. Regression of Default Probability Measures on Firm Characteristics........ 174

Table 7. Forecast Regression of Residual Cash................................. 177 


\section{LIST OF FIGURES}

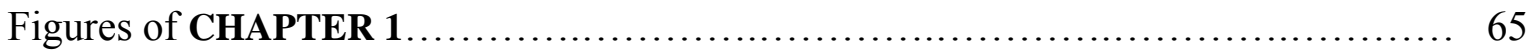

Figure 1. Time Series of Corporate Liquidity............................. 65

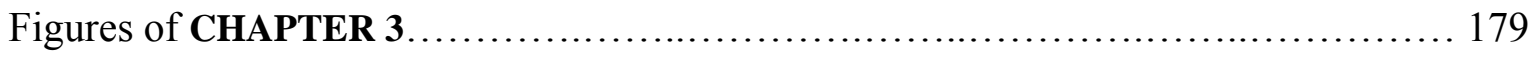

Figure 1. Time Series of Normal Cash and Residual Cash........................ 179

Figure 2. Bankruptcies and Failures by Year................................ 180

Figure 3. Bankruptcies and Failures as Percentage of Active Firms............... 180 


\section{Three Essays on Corporate Liquidity, Financial Distress and Equity Returns: An Introduction}

Corporate liquidity, as every manager knows, is the lifeblood of a business. Keynes (1936) provided three motives for individuals to hold cash. ${ }^{1}$ They hold cash for transaction purposes, as a precaution against unexpected future expenditure and for speculation. It is natural to extend these motives to firms since firms need liquid assets to maintain daily operations, to cover unanticipated expenses and reduce the probability of financial distress, and to quickly take advantage of future profitable investment opportunities when external financing is expensive. Moreover, the separation of management and ownership creates the additional incentives for the managers to hold more cash. Time series data show that the percentage of cash in total assets has been increasing dramatically during recent years, especially for those most liquid firms. Some firms hold large sums of liquid assets such as cash and marketable financial securities. In 2004, Ford held $\$ 32.9$ billion and Microsoft held $\$ 60.6$ billion in cash and marketable securities. ${ }^{2}$ Other firms like WalMart and DuPont consistently keep a lower level of cash.

Are "excessively" high cash holdings justified? How does the stock market value the cash held by firms? How will corporate liquidity affect the equity returns? Does corporate liquidity represent as an idiosyncratic or systematic risk? Is a low-than-optimal level of corporate liquidity related to financial distress even default and bankruptcy? These are all interesting questions to address.

The purpose of this dissertation is to empirically analyze the relationship between corporate liquidity and expected equity returns, whether corporate liquidity represents an idiosyncratic source of risk, or serves as a priced risk factor, the extent to which corporate liquidity can impact a firm's value, and the probability of financial distress even bankruptcy.

Corporate finance literature has extensively studied the motives of holding liquid assets (Miller and Orr (1966), Kim, Mauer and Sherman (1998), Opler, Pinkowitz, Stulz and Williamson (1999) and Acharya, Almeida and Campello (2006), etc.), various firm

\footnotetext{
${ }^{1}$ Keynes, J.M., 1936. The General Theory of Employment, Interest and Money. Harcourt Brace, London

${ }^{2}$ The figures reported here are reported in the Compustat Industrial Annual Files.
} 
characteristics as determinants of the cash level (Vogel and Maddala (1967), Kim, Mauer and Sherman (1998), etc.), and whether there is an optimal level of liquid assets (Kim, Mauer and Sherman (1998), Opler, Pinkowitz, Stulz and Williamson (1999), etc.). However, the impact of cash holding on expected equity returns hasn't gained enough attention. This is particularly important because holding corporate liquidity can bring both benefits and costs, based on which a firm chooses to keep a certain level of financial liquidity. These benefits and costs will be reflected in the firm's market value and therefore its equity returns.

The first chapter examines whether and how cash holdings can affect the equity returns for individual firms. It is found that firms that hold a higher proportion of liquid assets have significantly higher expected equity returns than firms with low liquidity even after controlling for the size and value effect. This can be interpreted as precautionary saving signals potential risks and leads to higher expected return, if we can find evidences showing relevant risk information associated with corporate liquidity.

Three types of potential risks are examined. First, firms whose future cash flows are more volatile tend to hold more cash and have higher stock returns compared to firms with more stable cash flows. This is consistent with Opler, Pinkowitz, Stulz and Williamson (1999), where firms with volatile cash flows have more cash. If a company accumulates a high level of financial slack out of a precautionary purpose as the managers have such information that firm's cash flow risks will be greater in the future, required equity returns will be higher as the high corporate liquidity signals this risk. Second, the positive relationship between equity returns and corporate liquidity is more pronounced for firms with financial constraints. Opler, Pinkowitz, Stulz and Williamson (1999) find that firms with easy access to financial market tend to hold less cash while Pinkowitz and Williamson (2004) point out that cash holding is more valuable for financially constrained companies. Hence a reasonable explanation for the distinct positive relation between corporate liquidity and equity returns among financially constrained firms is that firms stock liquid assets to lower down financial constraint risk, and a high level of cash signals this motive and implies higher a financial constraint risk. Furthermore, the empirical evidence shows that corporate liquidity is positively related to 
beta, which implies that a higher level of financial slack indicates risks and might serve as a risk factor.

Finally, results of Fama-MacBeth two-pass regression suggest that corporate liquidity contains information different from what is contained in important firm characteristics such as size and book-to-market equity since the impact of corporate liquidity on equity returns remains significant even after these characteristics are controlled for. This extends support to the view that corporate liquidity contains certain risk information and its observed level therefore signals the potential risks.

The second chapter investigates the relationship between aggregate corporate liquidity and the expected return of stocks from an asset pricing point of view. Corporate liquidity has been considered and studied as an idiosyncratic firm characteristic in previous corporate finance literature, which focus on the motive and determinants of liquid assets. However, how corporate liquidity can affect a firm's value and therefore equity returns have not been investigated extensively until recently. Pinkowitz and Williamson (2004) find that cash is more valuable for firms with financial constraints, better growth opportunities, more volatile investment opportunities and a lower probability of financial distress. Faulkender and Wang (2006) provide evidence that the market rewards firms holding internal cash with higher values, while the marginal market value of cash declines as the level of cash increases. They also find that on average, the market value of cash is higher for financially constrained firms than for those that are not constrained.

The asset pricing literature, on the other hand, tries to explain the asset returns with risk factors. Most of the asset pricing literature studying liquidity refer to financial liquidity, and they find liquidity is not only an idiosyncratic phenomenon, instead, aggregate liquidity can be a systematic risk factor ((Amihud and Mendelson (1986), Chordia, Roll and Subrahmanyam (2000), Pástor and Stambaugh (2003) and Acharya and Pedersen (2005)). In particular, Holmström and Tirole (2001) make adjustments to asset pricing theory using a corporate finance approach. They develop an asset pricing model based on firms' desire to hoard financial slack. Collateralizable assets require a premium determined by the covariation between the asset's return and the marginal value of 
liquidity $^{3}$ in different states. Risk neutral firms pay a premium on assets that can help them through the time when liquidity is in shortage. According to their model, asset prices are completely determined by corporate demand for liquidity.

The second essay examines whether corporate liquidity is a risk factor and whether it is systematic and priced. If aggregate corporate liquidity contains information about changes in future investment opportunities, it can proxy a state variable and can be used as a factor to price equity returns. I construct a corporate liquidity factor LMI (Liquid Minus Illiquid), which is a zero-investment portfolio long stocks with the most liquid assets and short stocks with the least liquid assets. Asset pricing tests are performed on various portfolios including 25 Size-BE/ME portfolios, 27 Size-BE/ME-Corporate Liquidity Portfolios and 10 corporate liquidity sorted portfolios.

Empirical results from estimating multifactor asset pricing models suggest that although the three popular factors $M K T, S M B$ and $H M L$ share some information contained in the corporate liquidity factor, $L M I$ can still captures abundant equity return variations across the portfolios and serves as a priced independent factor. Compared to CAPM and Fama-French three-factor model, the four-factor regression better explains the stock return variations across firms with different cash holding levels.

More empirical tests are completed to investigate whether the corporate liquidity factor is systematic and what kind of risk it may proxy for. It can be found that a negative movement in macroeconomic condition increases the corporate liquidity beta, with a larger magnitude for liquid firms relative to illiquid ones. Firms with higher corporate liquidity are riskier than less liquid firms because their higher sensitivity to the macroeconomic changes signals higher risks. Finally, there is evidence showing that the corporate liquidity beta of financially constrained companies is more sensitive to changes in macroeconomic situations and is greater in absolute value than for firms without financial constraints. These findings offer evidence to support the view that firms increase asset liquidity because they are financially constrained and therefore more exposed to macroeconomic risks.

\footnotetext{
${ }^{3}$ Liquidity in Holmström and Tirole (2001)'s paper refers to the aggregate value of financial securities that can transport wealth over time and back up future payments.
} 
Tradeoff theory proposes that firms should keep an optimal level of cash in order to maximize shareholders' wealth. A deviation from this level will decrease the firm value. However, in the real business world, firms are always observed to maintain a liquid asset higher or lower than the optimal level. Motivated by this inconsistence, the third chapter looks at the impact of residual cash on a company's market equity value and the ability of corporate liquidity and negative residual cash to predict bankruptcy.

Previous literature focuses more on the positive deviation of cash from the "optimal" or "normal" level and relates the positive residual cash issue to firm control and corporate governance since it is generally agreed that excessively high liquidity is associated with agency problems ${ }^{4}$. Faleye (2004) finds that proxy-fight targets keep more cash than nontarget firms. Dittmar and Mahrt-Smith (2007) show that positive excess cash is more valuable for firms with sound corporate governance than poorly governed firms. They also find that poorly governed companies dissipate cash quickly and in a value-destroying manner.

On the other side, Opler, Pinkowitz, Stulz and Williamson (1999) and Sibilkov (2005) show that firms hold negative residual cash because they are financially constrained and they do not find evidence to support the agency theory. Moreover, cash holding has been long used as an important input variable in financial distress or bankruptcy prediction function (Altman (1968), Zmijewski (1984), Kim, Mauer and Sherman (1998) and Campbell, Hilscher and Szilagyi (2005)), yet whether residual cash can predict bankruptcy remains unclear.

The third chapter starts with estimating the residual cash using a regression approach following Opler, Pinkowitz, Stulz and Williamson (1999) and Dittmar and Mahrt-Smith (2007) and a portfolio approach. It is found that on average firms with negative residual cash are smaller, less profitable, more heavily leveraged but have weaker ability to pay off debt, spend more in research and development, distribute less dividends and lack good governance.

\footnotetext{
${ }^{4}$ Myers and Rajan (1998) argue that corporate liquidity can be a source of agency problems. Dittmar, Mahrt-Smith and Servaes (2003) claim that agency problems are an important determinant of corporate cash holdings, and find that corporations in countries with poor shareholder protections hold more cash than countries where shareholder rights are well protected. Pinkowitz, Stulz and Williamson (2005) use cross country data to test the agency theory. They find that minority shareholders value cash holdings less in countries where investor protection is poor than in other countries, which provides supportive evidence for the argument that agency problems play an important role in firm valuation across countries.
} 
Value regression results show that either a positive or negative deviation from the target or normal cash is value destructive, which provides support to the tradeoff theory. However, the effect of residual cash on market value of equity is asymmetric such that market value of equity declines with a smaller magnitude for a larger positive residual cash compared to negative residual cash.

Furthermore, logistic regression results indicate that negative residual cash is an important predictor variable for bankruptcy. Given the corporate liquidity position, holding a less-than-normal-level of cash will increase the probability to file bankruptcy.

Finally, negative residual cash contributes to various default probabilities (Altman's Z-score, Vassalou and Xing's default likelihood indicator DLI and Chava and Jarrow's default probability) estimated in previous literature.

In summary, these essays contribute to the literature of corporate liquidity, multifactor asset pricing models and bankruptcy prediction in several important ways. First, corporate liquidity is positively related to the expected equity returns and contains important risk information other than size and book-to-market equity, and therefore can signal potential risks for individual companies. Second, corporate liquidity effect remains significant even after market, size and value factor have been controlled for, implying the existence of corporate liquidity factor, which contains risk information about changes in future investment opportunities. Indeed, corporate liquidity factor $L M I$ has done a great job interpreting equity return variations across portfolios created based on corporate liquidity holding. Third, a deviation from the optimal or normal cash level is value destructive, with a larger magnitude for negative residual cash. Negative residual cash serves as an important predictor for firm bankruptcy.

The dissertation accordingly consists of three chapters. Chapter 1 studies idiosyncratic corporate liquidity and its relationship with expected equity returns. Chapter 2 examines whether the aggregate corporate liquidity can serve as a risk factor and be priced in multifactor asset pricing models. Chapter 3 investigates the role of residual cash in affecting market value of equity and predicting bankruptcy probability. 


\section{Chapter 1 \\ Idiosyncratic Corporate Liquidity and Equity Returns}

\section{Introduction}

Corporate liquidity is an important issue widely studied in corporate finance. If a firm can be considered as an asset, then corporate liquidity shares some features in common with financial asset liquidity in the sense that they both refer to the ease and speed of conversion into cash. Some firms hold large sums of liquid assets such as cash and marketable financial securities. In 2004, Ford held \$32.9 billion and Microsoft held \$60.6 billion in cash and marketable securities ${ }^{5}$. Other firms like Wal-Mart and DuPont tend to keep a low level of financial slack.

Holding liquid asset brings benefits to firms, and these benefits also serve as motives for holding cash. (1) The transaction cost motive: Firms need financial slack to maintain daily business without having to frequently raise funds from external capital markets, saving transaction costs (Miller and Orr (1966), Faulkender (2004)). (2) Precautionary motive: Firms stock cash in case they are forced to liquidate assets to make payments during hard times, reducing the probability of financial distress. Specifically, firms hold financial slack when general economy is volatile (Custodio and Raposo (2004), Baum, Caglayan, Ozkan and Talvera (2004)), when their cash flows are unpredictable and volatile (Kim, Mauer and Sherman (1998), Opler, Pinkowitz, Stulz and Williamson (1999)), and when their financial leverage is relatively high given their operating cash flow (Kim, Mauer and Sherman (1998), Opler, Pinkowitz, Stulz and Williamson (1999), Damodaran (2005)). (3) Speculative motive: Firms hold liquidity reserve in order to quickly take advantage of future profitable investment opportunities in case they are financially constrained (Baskin (1987)). Firms need to set aside cash for speculative purpose when future investments are uncertain (Acharya, Almeida and Campello (2006)), when alternative internal financial sources are not sufficient while external sources of funding are expensive or unavailable (Pinkowitz and Williamson (2001)), and when information asymmetry problem makes it difficult to raise capital at a fair price (Myers

\footnotetext{
${ }^{5}$ The figures reported here are obtained from Compustat Industrial Annual Files.
} 
and Majluf (1984)). Moreover, according to agency theory, the separation of management and ownership creates the additional incentives for the managers to hold more cash since liquidity can increase managers' discretion and shield management from market discipline (Opler, Pinkowitz, Stulz and Williamson (1999)).

However, maintaining asset liquidity incurs costs as well: (1) By choosing to hold liquid assets, firms receive a lower rate of return and forgo an opportunity to invest in illiquid and riskier projects (or more productive assets) that can bring higher future returns. (2) Holding cash equivalents brings tax disadvantages. Interest income earned on liquid assets is double taxed both at the corporate and individual level as it is paid to investors as dividends (Opler, Pinkowitz, Stulz and Williamson (1999)).

Although corporate finance literature has intensively examined cash holding as an internal resource, the impact of cash holding on expected equity returns hasn't gained enough attention. This is particularly important because holding corporate liquidity can bring both benefits and costs, based on which a firm chooses to keep a certain level of financial liquidity. These benefits and costs will be reflected in the firm's market value and therefore its equity returns.

Corporate finance has developed theories and models of companies' motives and behavior concerning liquid assets and their relationship with the firm's capital structure. According to tradeoff theory, there should be an optimal level of liquid asset holdings on a corporate balance sheet that can maximize the stockholder's value. Kim, Mauer and Sherman (1998) model the firm's decision to invest in liquid assets when external finance is costly. They predict that the optimal level of investment in liquidity increases with external financing cost, the variance of future cash flows, and the return on future investment opportunities; while optimal investment in liquidity decreases with the return differential between the firm's physical assets (ROA) and the liquid assets (Treasury securities' returns).

Agency theory says that managers prefer holding more cash and other liquid assets since asset liquidity can reduce the firm's risk and increases their discretion, which, as a result, leads managers to place much more importance on the precautionary motive for holding cash. This theory provides an explanation for why firms do not keep cash at the level that could maximize the shareholders' wealth. 
On the other hand, asymmetric information theory suggests that a higher degree of information asymmetry results in a higher cost of external finance, and hence more cash holdings. Myers and Majluf (1984) propose that firms whose values depend more on growth opportunities suffer more severe asymmetric information problems and are more likely to be financially constrained. In addition, small firms usually suffer more from information asymmetry problems, probably because they are not well known and not many financial analysts study these firms. This suggests that small firms and growth firms tend to keep a higher level of financial slack.

Opler, Pinkowitz, Stulz and Williamson (1999) find evidence to support the static tradeoff model of cash holdings. Specifically, Firms with stronger growth opportunities (a high market-to-book ratio and R\&D expenditures) and riskier cash flows hold more cash as a proportion of total non-cash assets. Firms with the greatest access to the capital markets, such as large firms and those with higher credit ratings, tend to keep a lower level of cash. Companies tend to accumulate more cash than the level predicted by tradeoff theory at which the shareholders' wealth is maximized, which provides evidence of an agency problem.

Gertler and Hubbard (1998) and Whited (1992) show that small firms are likely to face more stringent constraint for raising funds. If transaction costs of small firms are higher, which indicates the illiquidity of these firms, investors will require a higher return on equity, then small firms have to accumulate a larger portion of liquid assets and more frequently use internal funds to finance investment. Kim, Mauer and Sherman (1998) use size and market-to-book ratios as proxies for external financing costs. Since large firms tend to have lower fixed costs of issuing securities due to economies of scale as well as fewer borrowing constraints and easier access to capital markets, it is reasonable to predict a negative relation between firm size and liquid assets investment. Furthermore, Vogel and Maddala (1967) find that cash-to-assets and cash-to-sales ratios are generally lower for larger manufacturing firms, and that cash holdings declined over time, which is interpreted as evidence of economies of scale in transaction costs of cash holding. We would expect that small firms have higher asset liquidity, as well as a lower leverage ratio, compared to large firms. On the other hand, pecking order theory says that the primary determinant of the corporate financing decision is the information asymmetry of the 
firm's quality between insiders and outsiders. In this sense, small firms suffer more from the information asymmetry problem and have to keep a higher level of cash for precautionary purposes. In general, it is expected that there is a negative relationship between firm size and liquid asset holdings.

It is also interesting to see how the book-to-market equity ratio is related to corporate liquidity. Generally, value firms tend to be more mature firms which generate stable revenue but do not have substantial growth opportunities in which they may invest their revenue. Those firms may pay dividends regularly, but accumulate cash after making all the payments. Although they have easier access to the capital market, they could also retain a substantial amount of cash because of a lower opportunity cost of holding more liquid assets. If a value firm holds little cash, this could be a signal of financial distress, which may be because it cannot generate enough revenue to pay back its debt and other liabilities.

On the other hand, if high $\mathrm{BE} / \mathrm{ME}$ (book-to-market equity ratio) reflects less information asymmetry, then value firms should have a lower cost of external finance and an easier access to the capital market. These firms are able to raise external funds when they need, and need not keep a high proportion of their assets in liquid form.

Growth firms are young, risky, and have more growth opportunities on average, and therefore have limited debt capacities that are easily exhausted. As a result, these firms hold less cash because they probably have invested most of their funds. If, however, these firms are found not to face credit constraints, while still keeping high cash levels, these firms might not have abundant growth opportunities. We can expect that growth firms with higher levels of liquid asset holdings may be overvalued, and their future rate of return may be lower. It can be argued that a lower average return of a growth firm may be due to lower loadings on risk factors, but if there is no such risk factor, the firm is just overvalued.

An opposite view suggests that there is a positive (negative) link between market-tobook ratio (book-to-market equity) and liquid asset holdings. Due to the existence of risky debt financing, managers have the incentive to underinvest in growth options and keep excess liquid assets in order to reduce the possibility of financial distress (Myers (1977)). Shleifer and Vishny (1992) point out that financial distress is more costly for 
firms with a large proportion of intangible assets ${ }^{6}$ or with more growth options. As a result these firms tend to keep a relatively large proportion of liquid assets to reduce the financial distress cost. John (1993) finds that firms with high market-to-book ratios as well as those with relatively low tangible assets tend to hold more cash. All of these views support the positive relation between the market-to-book ratio and liquid asset holdings. If the financial distress theory is correct, where the market-to-book ratio or book-to-market equity serves as a proxy for financial distress costs, then firms with more intangible assets hold more cash in case they incur high financial distress costs.

In summary, value firms with low cash holdings are probably in financial distress; while growth firms with high cash holdings could be over valued. If this is true, low cashholding value firms should generate a higher rate of return than high cash-holding value firms; and high cash-holding growth firms should generate a lower rate of return than lower cash-holding growth companies. Although there appears to be a relationship between the book-to-market equity and cash holding, it remains unclear that whether the information contained in the book-to-market ratio is the same as that is included in the cash reserve.

Although previous literature has established that the cash holding is important for a firm's investment and operation, it is not clear whether cash holding can enhance the value of firms. Mikkelson and Partch (2003) find that in the long run, firms that retain high cash reserves grow faster and undertake high levels of investment, and they conclude that conservative financial policy can enhance the operating performance. Pinkowitz and Williamson (2004) point out that cash holding is more valuable for financially constrained companies as well as firms with better growth opportunities and more volatile investment opportunities. Faulkender and Wang (2006) adopt a methodology that examines the variation in excess equity returns as a measure of marginal value of cash. They find that the market rewards firms holding internal cash with higher values, while the marginal market value of cash declines as the level of cash increases. They also find that on average, the market value of cash is higher for financially constrained firms than those that are not constrained. Sibilkov (2005) finds

\footnotetext{
${ }^{6}$ Intangible assets include copyrights, patents, intellectual property, and goodwill. Goodwill is a blanket term that represents, in one lump sum, the value of brand names, patents, customer base loyalty, competitive position, $\mathrm{R} \& \mathrm{D}$, and other hard-to-price assets a company might own.
} 
that cash holdings are more valuable for financially constrained firms, partly because constrained firms use cash more efficiently to invest in more valuable projects than financially unconstrained firms.

In microstructure literature, the financial liquidity refers to the speed and ease with which an asset can be converted into cash (Glossary of Bodie, Kane and Marcus $4^{\text {th }}$ edition, 1999). Amihud and Mendelson (1986) examines the role of the bid-ask spread as a liquidity measure in determining asset returns and predicts that expected asset return is an increasing and concave function in the relative bid-ask spread. One implication of the model is that firms can reduce the opportunity cost of capital by implementing financial policies that can increase the liquidity of their securities, and thereby increasing the firms' value.

Several studies try to provide interpretations for the size effect in terms of illiquidity. Stoll and Whaley (1983) find that the small firms tend to have higher bid-ask spreads. They show that transaction costs, representing a missing factor from the CAPM, can partially explain the small firm effect. They argue that the CAPM should be applied to the return net of transaction costs. Schultz (1983) also finds that small firms tend to have higher transaction costs; however, he concludes that transaction costs cannot fully explain the high average returns to the stocks of small firms.

A substantial body of asset pricing literature addresses the issue of financial liquidity, however, there has been little focus on corporate liquidity. If firms keep asset liquidity to prevent financial distress risk, to avoid high transaction costs of accessing external financial market, or to invest in value-enhancing investments, this corporate liquidity effect could be reflected in the firms' stock prices as well as the expected returns on their equity. If companies stock financial slack for the precautionary purposes, due to the asymmetric information, investors would take the higher level of cash holdings as an indication of future volatile cash flows or decreasing profitability of the firms, therefore require a higher return on the equity. In addition, if a company is financially constrained, accumulation of internal resources is even more important. Given investors are rational and information is asymmetric, a high level of financial liquidity will signal the constraint risk and is associated with a greater required rate of return. 
Now we have a better understanding of the firm characteristics that determine the level of liquid assets that a firm keeps, it is tempting to further explore how the market values can be affected by the cash holdings of firms, and how the expected equity returns are related to corporate liquidity. It is interesting to ask questions such as: How do liquidity positions of large and small firms affect their expected returns? Do firm cash holdings have different implications for value firms and growth firms? How does corporate liquidity relate to stock market anomalies like the size and book-to-market effect? Does corporate liquidity share similar information with size and book-to-market equity? Does corporate liquidity explain stock return variations beyond the size and value effects?

This study tries to explore the effect of individual corporate liquidity on the stock returns. It can be shown empirically that firms that hold a higher proportion of liquid assets have higher expected returns than firms with low liquidity, which seems to be counter to the standard risk-based theory at the first glance, where lower holdings of cash may indicate financial distress. I also find that firms' whose future cash flows are more volatile tend to hold more cash and have higher stock returns compared to firms with more stable cash flows. In addition, evidence shows that the positive relationship between equity returns and corporate liquidity is more distinct for firms with financial constraints. Furthermore, the empirical evidence shows that corporate liquidity is positively related to beta, which indicates that higher level of financial slack indicates risks and might serve as a risk factor. Finally, regression results suggest that corporate liquidity contains information different what is contained in important firm characteristics such as size and book-to-market equity since the impact of corporate liquidity on equity returns exist even after these characteristics are controlled for.

The remainder of the paper is organized as follows: section II discusses the hypothesis to be tested in the empirical estimation; section III describes the data and variables; section IV discusses the empirical findings, and section V concludes.

\section{Hypothesis}

The previous literature has shown that firm characteristics, such as size, book to market ratio, leverage and whether the firm is financially constrained, are closely related 
to the future stock returns. Whether corporate liquidity, an important characteristic for individual firms, represents or indicates an idiosyncratic risk and affects the equity returns remains unclear.

If a company accumulates a high level of financial slack for precautionary purpose because the managers know that the firm's cash flow risks will be greater in the future, expected equity returns will be higher for this company if information is symmetric. Even if managers know the insider information and information is asymmetric, given investors are rational, they would know that the observation of large financial reserves signals future cash flow fluctuations and will require higher returns on the firm's stocks. This leads to the first hypothesis:

\section{Hypothesis 1: Higher corporate liquidity is associated with higher expected} stock returns if future cash flows are more volatile.

One important motive for holding liquid assets is to speculate on profitable investment opportunities, and this is especially important when the firm is financially constrained. A firm is financially constrained if it is unable to borrow or to issue stocks, or it can only raise external capital at a high cost. Previous literature has provided evidence that firms stock more financial slack if they have difficulty in accessing capital market (Pinkowitz and Williamson (2001)). Almeida, Campello and Weisbach (2004) present a model in which companies respond to potential financial constraints by hoarding more cash today. Furthermore, Lamont, Polk and Saá-Requejo (2001) find that stock returns of financially constrained firms tend to move together, which indicates that these firms are subject to common shocks. Campello and Chen (2005), Whited and Wu (2006) show that constrained firms earn higher returns and external finance constraints do indeed represent a risk factor that is priced in financial markets. If financial constrained firms are riskier, which is the reason for these firms to maintain a high level of liquidity, such firms will have higher expected stock returns. The relationship between corporate liquidity and equity returns should be more pronounced for companies that are financially constrained. This can be tested by the second hypothesis: 
Hypothesis 2: Higher corporate liquidity is associated with higher expected stock returns if the firm is financially constrained.

From the asset pricing point of view, idiosyncratic risk is the portion of the variability in stock returns not related to overall movements in the general market or economy. This risk is unique to a particular security and is associated with such factors as business and financial risk as well as liquidity risk.

According to the capital asset pricing model, beta is a measure of the systematic risk of a security that cannot be diversified through diversification. Beta is a relative measure of risk - the risk of an individual stock relative to the market portfolio of all stocks. A firm characteristic unique to a specific company would represent a risk if they are correlated with the market factor. If higher cash holding indicates possible higher risks for firms in the future, such as financial constraint risk, low or volatile cash flows risk, then it can be expected that corporate liquidity is positively related to future betas.

Hypothesis 3: Corporate liquidity is positively correlated with firm beta if liquid asset holding signals a higher level of future risk for the firm.

Finally, although empirical research in general agrees that size and value effects are real, there is no agreement on what kind of underlying risk that firm size proxies for. Previous literature also discusses the relationship between cash holding level and size or book-to-market equity ratio. If the information incorporated in the corporate liquidity is similar to that in firm size and book-to-market ratio, corporate liquidity will disappear after size and value effects are controlled for. Otherwise, we would expect the corporate liquidity effect remains existing even when the size and value effects are removed.

Hypothesis 4: If corporate liquidity contains information different from that in firm size and book-to-market equity, corporate liquidity will exist even after size and book-to-market are controlled for. 
The above four hypothesis are tested through statistics obtained from portfolio construction as well as formal regressions. The results are presented in part IV.

\section{Data}

To examine the effect of corporate liquidity on stock returns, I construct a sample of firms for the empirical tests by merging the COMPUSTAT annual files and the stock and indices databases from the Center for Research in Security Prices (CRSP) for the period 1962:1 to 2005:12. The CRSP returns cover all NYSE, AMEX and NASDAQ stocks. I exclude financial firms with Standard Industrial Classification (SIC) codes between 6000 and 6999 because they usually hold substantial inventories of marketable securities, which are included in cash. Transportation and public utility firms having SIC codes between 4000 and 4999 are excluded as well because cash holdings of these firms are subject to regulatory supervision.

The individual firms' monthly stock returns are taken from CRSP. As the risk free interest rate for the asset pricing model, I use monthly observations of the one month Treasury bill rate obtained from Professor Kenneth French's webpage. ${ }^{7}$ Market excess returns are downloaded from professor Kenneth French's webpage as well.

I follow Kim, Mauer and Sherman (1998) and measure corporate liquidity as the ratio of cash and marketable securities (Compustat item \#1) to total assets (Compustat item \#6):

$$
C L_{i t}=\frac{\text { Cash }_{i t}}{\text { Total Assets }_{i t}}
$$

where Cash $_{i t}$ includes the following items: bank drafts, cash, checks (cashiers or certified), demand certificates of deposit, demand deposits, letters of credit and money orders. It also includes government and other marketable securities and time deposits, which are usually considered as cash equivalents. A firm with a high cash-to-asset ratio has more liquid assets to serve interest payments, and therefore postpone bankruptcy and reduce the financial distress; or has more freedom to invest in profitable projects without incurring transaction costs. Alternatively, corporate liquidity is measured by the ratio of cash and cash equivalents to net assets, where net assets equal total assets less cash and

\footnotetext{
${ }^{7}$ http://mba.tuck.dartmouth.edu/pages/faculty/ken.french
} 
cash equivalents (Dittmar, Mahrt-Smith and Servaes (2003), Faleye (2004), etc.). Following Campbell, Hilscher and Szilagyi (2005), I also measure liquidity using cash, marketable securities and short term investment in ratio to total assets, since short term investment is often quite liquid as well. The results are similar when the two alternative measures of corporate liquidity are used.

The size of a firm is measured by the market value of equity, which equals the stock price of the stock at the end of June of year $t$ times the total shares outstanding. Following Fama and French (1992), the book-to-market equity ratio (BE/ME) at time $t$ is calculated by dividing book equity (BE) at the fiscal ending of year $t-1$ by market equity (ME) at December of year $t-1$. The book-to-market equity ratios at time $t$ are matched by the stock returns from July of year $t$ to June of year $\mathrm{t}+1$. Therefore there is at least a 6 months' lag between accounting data and market data in order for the firms' accounting information to be released and understood by the public. The book value of firm equity is defined as in Cohen, Polk and Vuolteenaho (2003). ${ }^{8}$ Following Opler, Pinkowitz, Stulz and Williamson (1999), I calculate leverage as (long-term debt + short-term debt)/book value of assets. ${ }^{9}$ Cash flow is calculated as "earnings before extraordinary items and depreciation after dividends" ${ }^{10}$, following Almeida, Campello and Weisbach's approach. Cash flow volatility for each firm is measured as standard deviation of cash flow over total assets.

There are various measures for financial constraints. I use three alternative measures suggested by the literature. (1) Asset size. Gilchrist and Himmelberg (1995) argue that smaller firms are more likely to be subject to financial constraint because they are usually young and less well known, hence have difficulty in accessing external capital market. (2) $\mathrm{KZ}$ index, which is a linear index of firm financial constraints constructed based on

\footnotetext{
${ }^{8}$ Book equity is defined as the stockholders' equity, plus balance sheet deferred taxes (Compustat item \#74) and investment tax credit (Compustat item \#208), plus postretirement benefit liabilities (Compustat item \#330), minus the book value of preferred stock. Depending on availability, they measure the book value of preferred stock by the order of redemption (Compustat item \#56), liquidation (Compustat item \#10), or par value (Compustat item \#130). Stockholders' equity is measured by Compustat item \#216 or the book value of common equity (Compustat item \#60), plus he par value of preferred stock, or the book value of assets (Compustat item \#6) minus total liabilities (Compustat item \#181).

${ }^{9}$ Long term debt is measured by Compustat item \#9 and short term debt is measured by Compustat item \#5.

${ }^{10}$ Cash flow is calculated as income before extraordinary items (Compustat item \#18), plus depreciation and amortization (Compustat item \#14), minus preferred dividends (Compustat item\#19) minus common dividends (Compustat item \#21).
} 
Kaplan and Zingales (1997). Following Lamont, Polk and Saá-Requejo (2001), the KZ index is calculated using the following equation ${ }^{11}$ :

$$
\begin{aligned}
\text { KZindex }= & -1.002 \times \text { CashFlow }+0.283 \times Q+3.319 \times \text { Leverage } \\
& -39.368 \times \text { Dividends }-1.315 \times \text { CashHoldings }
\end{aligned}
$$

The $\mathrm{KZ}$ index is a linear combination of five accounting measures and it measures the likelihood of financial constraint. The higher the $\mathrm{KZ}$ index, the less cash flow, the higher leverage and lower dividend distribution and therefore the more constrained the firm is. (3) Dividend payout, which is calculated as the ratio of total dividends distributions to operating income. Fazzari et al. (1988) finds that firms with financial constraints distribute significantly less dividends. As a robustness check, I follow Almeida, Campello and Weisbach (2004) and use the total distribution or payout to shareholders measure as a proxy for the financial constraint. The "Distribution to shareholders" variable is defined as the sum of dividends and stock repurchases divided by operating earnings.

To reduce the impact of outliers, firm specific characteristics and other dependent variables are winsorized at the 1 percent tails of the entire sample. Firms with negative total assets, book value and market value of equity are deleted from the sample. I also delete firms with less than 36 monthly observations. There are 6908 firms and 528 months in the combined COMPUSTAT and CRSP dataset. Summary statistics are provided in Table 1 .

\section{Empirical Results}

\section{IV.1. Firm Characteristics and Corporate Liquidity}

In this section, I first present the relationships between firms' characteristics. Table 2 exhibits the correlations between size, book-to-market ratio, liquidity holdings, leverage, cash flows and financial constraint measures of the firm. In order to remove the effect of industry differentials, I also calculate the deviation of firms' cash holdings and financial leverage compared to the industry norm. I assign each firm to one of the Fama-French 17

\footnotetext{
${ }^{11}$ Tobin's Q, calculated as the market value of assets divided by book assets. Cash flow, dividends and cash holdings are all standardized by total capital
} 
industries based on its CRSP four-digit SIC code and the firm's liquidity and leverage deviation are estimated as the difference between liquidity and leverage of that firm and the mean level of the industry that the firm belongs to. If the firm holds more liquid assets compared to an average firm in the same industry, the firm can be considered as hoarding too much cash, possibly because it is expecting good investment opportunities while having difficulty raising external funds, or because it grows relatively slowly and cannot spend the cash it has accumulated. If a firm has a higher leverage ratio than an average firm in the same industry, the firm could be facing financial problem.

Panel A shows the overall correlation between firms' characteristics. The size and book-to-market ratio of a firm are only weakly correlated with its cash position, leverage, as well as deviations of cash holdings and leverage from industry standard. Firms' liquidity is negatively correlated with its leverage ratio, indicating that the more cash the firm has, the less debt it keeps. In addition, firms that generate more cash flows tend to accumulate more cash. In general, companies that are financially constrained keep higher level of liquid assets.

Panel B summarizes the time series average of the cross-sectional correlations between firms' features. It can be shown that cross-sectionally, size and book-to-market ratio are negatively correlated, while size and cash holdings are positively related. At any point in time, larger companies accumulate more cash and cash equivalents than smaller firms. Again, larger firms borrow less than smaller firms and average firms in the same industry. In addition, firms with larger market value tend to have less financial constraints. The book-to-market equity is negatively correlated with corporate liquidity as well as liquidity deviation, but positively related to leverage ratio and leverage deviation. Value firms tend to hold less cash while borrowing more than growth firms, which is consistent with the financial distress story and the information asymmetry theory. Furthermore, value firms are less financially constrained relative to growth firms. Companies with more financial constraints tend to be more liquid, which supports the speculative motive.

The cross sectional averages of time series correlations between the firms' characteristics are displayed in Panel C. Over time, firm size is negatively correlated with the book-to-market ratio and leverage but positively correlated with cash holdings. As time goes by, large firms tend to accumulate more and more cash while may not find 
profitable opportunities to invest. Consistent with Panel A and Panel B, large firms generate more cash flows and are less constrained. The book-to-market ratio is negatively correlated with leverage and cash flows. It seems that value firms face less financial constraints. Liquid companies have higher cash flows, borrow less, and are more financially constrained.

Next all the stocks are sorted into quintiles based on corporate liquidity, size and bookto-market ratio, then the average firm characteristics are obtained for each portfolio. Panel A of Table 3 exhibits the average monthly rate or returns, cash position, firm size and book-to-market ratio, leverage, cash flow, cash flow volatility, and three measures of financial constraints of the five portfolios sorted by firm's cash holdings. Average stock returns increase monotonically with the cash holdings of the firm. The average monthly stock returns for the most illiquid portfolio is only 0.731 percent, which is significantly lower than the most liquid portfolio. The return differential between the top liquid portfolio and the bottom liquid portfolio is 1.269 percent per month, which is economically significant. At first glance, this seems to contradict the risk-based story, according to which firms holding less liquid assets should be riskier and more vulnerable to financial distress and therefore have higher required rates of return. However there are several alternative possible explanations for the negative relationship between average stock return and firm liquidity. First, it is possible that those firms that hoard more liquid assets because they have riskier cash flows or higher betas, and therefore high risk is associated with higher expected returns. This is supported by the precautionary motive of hoarding cash. Second, since firms hold more cash instead of investing or distributing cash as dividends, investors might think these firms lack growth opportunities, lowering the current stock prices. Third, the companies hold more liquid assets because the managers expect high cost of external capital due to limited access to the capital market and investors would require higher returns in order to hold these liquid firms' equity, therefore we can observe a negative relationship between cash holding and expected equity returns from the data.

Except for the most liquid firms, average firm size increases as the cash holding proportion increases except for the most liquid firm, probably because large firms are usually more mature, already in the stable stage of their life cycles, and hence have 
relatively less investment opportunities where they can spend cash. It is interesting to see that firms with highest corporate liquidity are actually the smallest firms. Average bookto-market equity ratio increases along with the corporate liquidity except for the most liquid portfolio. The most illiquid firms have the lowest book-to-market ratio equal to 1.498. The book-to-market equity increases substantially and reaches its peak at 3.949, and then declines. Cash flow increases with corporate liquidity first but drops later, while the cash flow volatility rises with cash holding level monotonically, implying that liquid firms stock financial slack for precautionary purposes due to lower cash flows and more volatile cash flows. The relationship between the four measures of financial constraints and the liquidity portfolio indicates that firms with more corporate liquidity are those subject to more financial constraints. This is consistent with the speculative motive hypothesis.

Panel B shows the average portfolio characteristics when quintiles are formed by sorting stocks based on firm size. Average stock returns decrease along with the increase of firm size. Liquidity increases with the firm size except for the biggest companies. Both the smallest and largest firms seem not to be the most liquid firms. For small firms, this maybe because they have capital constraints and have plenty of investment opportunities to spend cash; for large firms, this might be because they have easy access to the capital market and do not need to hold excess liquidity. Larger companies have higher and more stable cash flows compared to smaller firms. Evidence also indicates that larger companies are less likely to have financial constraint problems. Panel C shows that when the sample is sorted into five portfolios by book-to-market equity, stock return increases from low to high book-to-market equity, which is consistent with the value effect. The return differential between the firms with the highest book-to-market equity ratio and the lowest book-to-market ratio is statistically significant. Corporate liquidity declines monotonically with the book-to-market ratio, which supports the view that growth firms need to stock more cash to take advantage of good investment opportunities because they normally have limited access to the external capital market and a higher cost of capital. The negative relationship between cash holding and the book-to-market ratio is also consistent with Myers (1977), Shleifer and Vishny (1992), and John (1993), if the bookto-market ratio can be viewed as a proxy for growth options or asset tangibility, since 
firms with more growth opportunities or intangible assets tend to hold a higher proportion of liquid assets in order to reduce financial distress costs. Value firms generate more cash flows than growth firms and the cash flow volatility is much lower for value firms. Panel A has shown that cash flows of liquid firms are more volatile, it seems that book-tomarket equity ratio proxies different risk. Finally, all the four financial constraint measures show that value firms are less constrained relative to growth firms.

\section{IV.2. The Corporate Liquidity Effect}

In order to separate the effects of size, book-to-market and liquidity on the firm's characteristics, I examine the size effect and value effect controlled by corporate liquidity (See Appendix for details). It can be shown that size effect and value effect remain exist when we control for the corporate liquidity. Market equity and book-to-market ratio contains some different information than corporate liquidity does. Will the relationship between corporate liquidity and equity returns disappear if firm size and book-to-market ratio are controlled for? Table 4 and Table 5 display variations of firms' characteristics across liquidity sub-portfolios when firm size or book-to-market ratio is controlled for. Firms are first sorted into five portfolios by size or book-to-market ratio, and then further

sorted into five liquidity groups. If corporate liquidity does affect the expected equity returns, we should expect to observe a clear relationship between corporate liquidity and equity returns even after firm size and book-to-market ratio are controlled for.

\section{IV.2.1 The Corporate Liquidity Effect Controlled by Size}

Table 4 exhibits the firm liquidity effect when firm size is controlled for. Panel A shows that within each size cohort, returns steadily rise with the firm's cash position. The more liquid assets the firm holds, the higher average returns it generates relative to firms of similar size, which implies that firm size does not explain liquidity effect. Furthermore, return differentials between liquid and illiquid companies are much larger for smaller firms than for larger firms, indicating that corporate liquidity effect is stronger for smaller companies. Since small firms are considered to be riskier than large firms, we would expect corporate liquidity effect is more pronounced for small firms if higher cash holdings can signal higher risks. According to Panel C, except for the largest firms, 
firms' equity size is fairly stable across liquidity sub-groups. For those biggst firms, although size varies substantially, no clear pattern can be found. Panel D displays the relationship between book-to-market ratio and corporate liquidity. Across the size portfolios, more liquid firms tend to have higher book-to-market ratio except for the most liquid group. It seems that firms hold more financial slack as they are subject to financial distress risk, if book-to-market ratio contains some information about the distress risk. On average, for each size portfolio, firms holding more cash and cash equivalents tend to have lower leverage ratios, which also tend to deviate negatively from industry norm, as shown in Panel E and Panel G. Panel H exhibits the cash flow pattern across firms with different cash positions. On average, the smallest firms generate negative cash flows. For smaller companies, cash flows tend to be lower for those accumulate more cash, except for the most liquid firms. This pattern is reversed for larger firms, where cash flows are higher form more liquid companies. Moreover, Panel I indicates that firms with more liquid assets actually have more volatile cash flows, regardless of their size. Companies may stock financial slack if the managers know that cash flow risk is high, which is consistent with the precautionary saving motive. Panel $\mathrm{J}$ shows that within each size group, the more liquid a firm is, the higher beta coefficient that firm has. This suggests that more liquid firms have higher market risk. I perform t-test to see whether the most liquid firms have significantly different beta compared to most illiquid firms. It turns out that for each size portfolio, average beta of the most liquid companies are significantly higher than the most illiquidity ones. I have done the $\mathrm{F}$ test for difference in $\beta$ to partially test Hypothesis 3. More specifically, F-tests are performed to test the following two null hypothesis (i) beta differences of all liquidity-size quintiles are not significant; (ii) beta differences of the most liquid and most illiquid-size quintiles are not significant. The Fstatistics are displayed at the bottom of Panel J. It can be shown that the F-statistic for beta difference of all the liquidity-size quintiles is 57.37 with $p$ value almost equal to zero, and therefore we conclude that betas of the firms within different size quintiles are statistically different across different liquidity portfolios. In addition, F test is performed to identify whether firm betas are statistically different between the most liquid firms and the most illiquid firms, and the $p$-value is equal to 0.0007 . We conclude that holding firm 
sizes, beta is statistically different across corporate liquidity portfolios, with the pattern that liquid firms have higher beta on average.

Finally, in order to test whether financial constraint risk plays a role in determining the level of financial slack, financial constraint measures across the 25 size-corporate liquidity portfolios are summarized in Panel K through Panel N. Total assets increase first with corporate liquidity and declines as firms hold more and more cash, which indicates that both extremely liquid and illiquid companies are financially constrained. The other three proxies for financial constraints - the $\mathrm{KZ}$ index, dividend payout, and distribution to shareholders display a more linear relationship across liquidity portfolios. Within each size groups except the smallest ones, firms that hold high level of cash and cash equivalents tend to be more financially constrained, which supports the speculative motive of saving since they have difficulty in accessing external capital market. Smallest firms seem to have the opposite pattern, firms that are hoard more liquid assets turn out to be less constrained. This might be because smallest firms as a group tend to have financial constraint problem, those with more liquid assets then suffer less.

\section{IV.2.2 The Corporate Liquidity Effect Controlled by Book-to-Market Equity}

Table 5 summarizes the effect of cash holdings controlled for the book-to-market ratio. Panel A displays the average rate of return of firms' stocks. We observe a monotonically positive relationship between the liquidity and rate of return. No matter weather a firm is a growth firm or a value firm, as long as it holds a higher level of liquid assets, it will bring shareholders a higher rate of return. The return differential between most liquid firms and most illiquid firms are significantly different, with the narrowest monthly spread equal to 1.059 percent and the widest spread equal to 1.65 percent. Panel $\mathrm{C}$ shows the average size when book-to-market ratio and liquidity are held constant. It can be shown that within each book-to-market equity cohort, more liquid firms tend to be smaller firms. Compared to large firms, smaller firms are less mature, subject to information asymmetry problem, and face more financial constraints and higher transaction costs, therefore they need to hoard more liquid assets in order to survive through hard times and exploit profitable investment projects. As can be seen from Panel $\mathrm{D}$, the relationship between liquidity and the book-to-market ratio exhibits no clear 
pattern except for the highest book-to-market portfolio, where more liquid firms tend to have higher book-to-market ratios, indicating that they are subject to distress risk. Panel E shows the average leverage across the $25 \mathrm{BE} / \mathrm{ME}$-liquidity portfolios. Within each $\mathrm{BE} / \mathrm{ME}$ portfolio, firms with more liquidity tend to borrow less. Similar to Panel G of Table 4, on average, firms that hold more cash tend to borrow less from external sources, no matter if they are growth firms or value firms.

According to Panel $\mathrm{H}$, controlling for book-to-market ratio, liquid firms seem to generate less cash flows. The relationship between average cash flow volatility and corporate liquidity is presented in Panel I. Again we observe a positive relationship, implying that firms hold more financial slack because of higher cash flow risk. Cash flow volatility differential between the most liquid and illiquid portfolios are statistically significant. Panel J summarizes the average beta across different liquidity groups. Within each book-to-market portfolio, firm beta increases monotonically with the liquidity. This result indicates that higher liquidity firms are associated with higher risks measured by beta. The beta difference between the most liquid firms and the most illiquid firms is highly significant, supported by the t-test. Panel J of Table 5 also reports the F-statistics for beta differential across liquidity-BE/ME portfolios. Specifically, F-statistic for beta difference of all Liquidity-BE/ME quintiles is 47.84 with the $p$-value equal to zero; Fstatistic for beta difference of most Liquid and illiquid-BE/ME quintiles equals to 73.52 and the $p$-value is 0.001 .

Panel $\mathrm{K}$ through Panel $\mathrm{N}$ display the financial constraint measures for the BE/MEcorporate liquidity sorted portfolios. The results are mixed. Total assets and $\mathrm{KZ}$ index suggest that growth, liquid firms as well as value, illiquid firms tend to be financially constrained. There is no linear, monotonic relationship between corporate liquidity and the dividend payout ratio or distribution to shareholders. According to the dividend payout ratio, liquid firms are more likely to be constrained, except for the lowest BE/ME portfolio, where illiquid firms are more financially constrained. This pattern is similar when the distribution to shareholder variable is used to measure financial constraint, except for the two portfolios of value firms.

Table 4 and Table 5 both provide evidence that corporate liquidity is related to the individual firm beta, even if we controlled for the important characteristics such as size 
and book-to-market ratio. In order to formally test whether corporate liquidity can serve as a proxy for firm beta, I have run regressions of beta on corporate liquidity, firm size and BE/ME. As it can be shown in Table 6, corporate liquidity contributes to interpreting firm beta in all the three regressions. The coefficients on the corporate liquidity are positive and highly significant for all the three regressions. The $R$-squared ranges from 9 percent to about 17 percent. Together with the information in Panel I of Table 4 and Table 5, we can draw a conclusion that corporate liquidity is positively correlated with firm beta, which makes it reasonable for cash holding to serve as a proxy for the underlying idiosyncratic risks.

\section{IV.3. Fama-MacBeth Two-Pass Regression}

To examine the impact corporate liquidity can impose on expected equity returns, I run Fama-MacBeth two-pass regressions on all the individual firms in the sample. In the first pass, a time series of excess portfolio returns are regressed on market factor, $S M B$ and $H M L$ using the following formula:

$$
R_{i, t}-R F_{t}=\alpha_{i}+b_{i}\left(M K T_{t}-R F_{t}\right)+s_{i} S M B_{t}+h_{i} H M L_{t}+\varepsilon_{t},
$$

where $R$ is the portfolio stock return, $R F$ is the 3-month T-bill rate, $M K T-R F$ is the market factor, $S M B$ is the size factor and $H M L$ is the value factor. In order to generate the portfolio returns, individual firms are sorted based on size and book-to-market independently to form 25 portfolios, or are sorted based on size, book-to-market and corporate liquidity independently to form 27 portfolios. Then individual firms are assigned the beta coefficients (derived in the first pass regression) of the portfolio which they belong to. I assume that a firm's betas are stationary.

For the second pass, excess stock returns of individual firms are regressed on their factor betas, as well as other variables such as corporate liquidity level, squared corporate liquidity level, and the interaction term of corporate liquidity and cash flow volatility, and interaction term of corporate liquidity and financial constraint measures. The baseline model is shown as follows:

$$
R_{i}-R F=\alpha+\beta_{1} b_{i}+\beta_{2} s_{i}+\beta_{3} h_{i}+\gamma C L_{i}+\varepsilon_{i}
$$


where $b, s$ and $h$ are the loadings of market factor, size factor and value factor respectively in the first pass regression, and $C L$ is the corporate liquidity. Several different models are estimated and the results are presented in Table 7.

The base model can be estimated to test Hypothesis 4. If Hypothesis 4 holds and corporate liquidity does contain information different from that in firm size and book-tomarket equity and corporate liquidity effect exists even after size and book-to-market are controlled for, then we can expect that the coefficient on corporate liquidity $\gamma$ should be significant. Furthermore, we would like to see whether corporate liquidity CL imposes a positive impact on equity returns, as suggested by the previous portfolio sorting.

In order to formally test Hypothesis 1, I include an interaction term of cash holding and cash flow volatility in the base model. If the impact of cash holding on equity return is stronger for firms with more volatile cash flows, we expect to see a significant and positive coefficient on the interaction term.

For Hypothesis 2, I estimate the base model with an additional interaction term of corporate liquidity and a financial constraint measure. If the positive relationship between corporate liquidity and equity return is more pronounced for financially constrained companies, the coefficient on the interaction term should be positive and statistically significant.

Panel A of Table 7 presents the results of second pass regressions on individual firms with firm betas derived from time series regressions on 25 size-BE/ME portfolios in the first pass. Model 1 presents the result of the base model which regresses equity returns on market beta, size beta and value beta. It can be seen the coefficients on the individual firm's market beta and value beta are statistically significant, while size beta does not have a significant coefficient in the second pass regression. Model 2 formally tested the impact of corporate liquidity on the equity returns. The result shows that the coefficient of corporate liquidity is positive and significant at 1 percent level. As a firm increases its cash holding by the 10 percent, its monthly equity returns increase about 0.15 percent, which is about 1.8 percent per year. Market beta and value beta do not lose their significance while size beta is still insignificant. It seems that corporate liquidity contains some different information than market portfolio and the book-to-market ratio. Tradeoff 
theory suggests that there should be an optimal cash level that will maximize the shareholders' value, thus the relationship between corporate liquidity and equity returns may not be linear. In order to test this, a squared corporate liquidity term is added to model 2 and the result is presented in Model 3. Regression results show that the squared corporate liquidity has a significantly negative sign, which provides support for the tradeoff theory, and the relationship between corporate liquidity and equity returns is concave.

Model 4 formally tests Hypothesis 1 . The coefficients on both corporate liquidity and the interaction term between corporate liquidity and cash flow volatility are positive and significant, which supports the Hypothesis 1 . Firms with more volatile cash flows tend to have a more pronounced relationship between cash holding and equity returns. Cash flow volatility plays an important role in explaining the positive impact of corporate liquidity on equity returns. As the previous evidence shows that firms that have highly volatile cash flows tend to hold more cash compared to those with stable cash flows, the positive link between corporate liquidity and stock returns can be attributed to the precautionary saving motive. Companies whose cash flows are not stable over time are subject to higher risks, thus managers may stock relatively more financial slack as a cushion. High cash holdings therefore can signal the cash flow volatility risk and lead to a higher required return on stocks. Furthermore, industry is a very important factor in determining the cash holdings. Some industries on average hold more liquid assets compared to others due to higher business risk or more financial constraints. Table 8 presents the distribution of firms across industries and average corporate liquidity and corporate liquidity variability for each industry. It can shown that those industries with higher average corporate liquidity are the ones have more volatile cash holdings, such as drugs, tobacco, machinery and business equipment. In order to control for industry effect, Model 5 include industry dummies, where industries are defined following Fama and French 17industry classification. The coefficients do not seem to be varying much compared to the regression without industry dummies, but the explanatory power of the regression is enhanced substantially.

Model 6 through Model 8 test Hypothesis 2 using different measures for financial constraints. If the financial constraint does impose firms to additional risks, firms ought 
to have the incentive to save more liquid assets in order to lower down the cost of seeking external financing. Previous empirical evidence from portfolio sorting shows that companies that face financial constraints tend to have higher cash holdings compared to those are less constrained. In this case hoarding financial slack signals the financial constraint risk faced by a company, therefore the relationship between corporate liquidity and stock returns should be strengthened for constrained firms relative to unconstrained firms. As a result we should observe a significant coefficient on the corporate liquidityfinancial constraint interaction term that indicates stronger relationship between cash holding and equity returns for financially constrained companies. Model 6 includes the interaction term between corporate liquidity and $\mathrm{KZ}$ index in the Fama-MacBeth secondpass regression. The coefficient on the interaction term is positive and significant. KZ index is higher for companies that are more financially constrained, and therefore a positive coefficient on the interaction term is consistent with the Hypothesis 2. Model 7 uses dividend payout ratio as a proxy for financial constraint. Companies that are not financially constrained tend to distribute more after tax earnings as dividends to shareholders, so that a higher dividend payout ratio indicates less financial constraint. We observe a statistically significant and negative coefficient on the corporate liquiditydividend payout ratio interaction term, providing evidence to support the view that firms have difficulty accessing capital market tend to save more cash to speculate on future investment opportunities or to prepare for the potential financial distress. As a robustness check, Model 8 uses distribution to shareholder as the financial constraint measure. It turns out that the regression result is fairly similar to that using the dividend payout ratio in the interaction term.

Panel B shows results of second pass regressions on individual companies with beta coefficients estimated from time series regressions on 27 size-BE/ME-corporate liquidity portfolios in the first pass. It can be shown that individual companies' cash holding positions strongly affect the expected returns of the stock. The coefficient of corporate liquidity is significantly positive for all the models in panel B, ranging from 1.245 to 3.452. Model 2 shows that when market beta, $S M B$ beta and $H M L$ beta are included in the regression, individual firm's liquidity position still affects the firm's stock returns substantially. For every 10 percent increase in the firm's cash holding, average monthly 
stock return will rise by 0.164 percent. Put in another way, for 1 standard deviation ${ }^{12}$ change in the firm's liquidity, monthly equity return changes for 0.31 percent on average. Model 3 shows that equity returns and corporate liquidity holding exhibit a quadratic relationship, with the coefficient of corporate liquidity variable positive and the squared corporate liquidity variable negative.

Model 5 tests Hypothesis 1 and provides empirical evidence that corporate liquidity is associated with higher equity returns if the firm has higher level of cash flow volatility. The empirical result is similar to the regression based on betas derived from 25 Size$\mathrm{BE} / \mathrm{ME}$ portfolios. The relationship between corporate liquidity and equity returns is stronger for firms with cash flow risks. Model 5 controls for industry effect, and the coefficient on the interaction term becomes much greater.

Model 6 and model 7 use KZ index and dividend payout ratio respectively as financial constraint measures to test Hypothesis 2. The coefficient on the interaction term of corporate liquidity and $\mathrm{KZ}$ index is significantly positive while the coefficient on corporate liquidity - dividend payout interaction term is significantly negative, consistent with the hypothesis that more constrained firms' cash position has greater impact on equity returns. Model 8 replaces the dividend payout ratio with distribution to shareholders as a robustness check. The value and significance of the coefficients do not change appreciably.

Fama-MacBeth second-pass regressions on individual firms formally test the hypotheses in question. The empirical evidence shows that companies holding more cash tend to have higher equity returns on average. The effect of corporate liquidity imposes on stock returns is statistically significant, even after market, size and value effect have been controlled for. I also find that the equity returns are more sensitive to corporate liquidity holding for companies with more volatile cash flows and companies that are financially constrained. This suggests that firms hoard high financial slack due to precautionary saving motive, and higher cash holding may signal higher cash flow risk and financial constraint risk. Investors consider corporate liquidity as a proxy for the relevant risks and require higher equity returns on more liquid companies.

\footnotetext{
${ }^{12}$ Standard deviation of corporate liquidity is 0.195 , as shown in Table 1.
} 


\section{Summary and Conclusion}

This paper examines the effect corporate liquidity may impose on the equity returns. We ask how corporate liquidity is related to the expected equity returns and whether the information embedded in corporate liquidity the same as in size and book-to-market equity ratio. At the first glance, the results seem to contradict the risk-based story that firms with lower cash holding are possibly in financial distress and therefore have higher expected stock returns. In fact, I find that firms that hold more liquid assets offer higher returns than firms with low liquidity. The results suggest that the market rewards firms that keep a higher level of liquidity with higher expected returns. However, these findings can be interpreted using asymmetric information and signaling theory. Firms stock higher financial slack since the inside managers know that future risks will increase, because information is asymmetric, rational investors will require higher future returns on liquid firms because they see higher level of corporate liquidity as a signal for riskier future cash flows and uncertain operating performance.

Furthermore, empirical evidence shows that even after controlling for size and value effect, corporate liquidity is positively correlated with firm beta. This implies that liquid asset holding serves as a signal of higher level of future risk for liquid firms. Regression results show that corporate liquidity contributes to interpreting beta and therefore can be used as a proxy for risks.

Models using the Fama-MacBeth two-pass regression approach and incorporating firm cash holding and its deviation from the industry average as an explanatory variable show that a higher level of firm liquidity or holding more cash than the average industry level will increase the expect stock returns. Empirical results show that corporate liquidity contains information different from that in firm size and book-to-market equity, since corporate liquidity effect exists even after size and book-to-market are controlled for. Furthermore, the effect of corporate liquidity on equity returns differs across firms with various cash flow volatilities and financial constraints. Specifically, the positive impact of corporate liquidity on expected equity returns are stronger for firms with more volatile cash flows and are financially constrained. Regression results support the precautionary saving motive for companies and signaling effect of corporate liquidity. Corporate 
liquidity may serve as a proxy for the potential volatile cash flow risk and financial constraint risk of the firms. 


\section{APPENDIX of Chapter 1}

The appendix first presents the empirical evidence of size effect and value effect after the corporate liquidity effect is controlled for; and then discusses some additional FamaMacBeth two-pass regression results.

The paper shows that there exists a significant corporate liquidity effect when size and value effect are controlled for. This suggests that the financial liquidity held by a company contains important information other than what is included in firm size and book-to-market ratio.

\section{A.1. The Size Effect Controlled by Corporate Liquidity}

In order to separate the effects of size, book-to-market and liquidity on the firm's characteristics, I first control for the corporate liquidity and examine how the average firm return, liquidity, size, book-to-market, average leverage vary with size and book-tomarket value. Table 1 shows the size effect controlled by firm liquidity. First, all the firms are sorted into five portfolios based on corporate liquidity, within each liquidity cohort, firms are further sorted into five portfolios by size. Panel A exhibits the variation of average stock returns when corporate liquidity is controlled for. For each of the liquidity portfolio, smaller firms tend to generate higher rate of return than larger firms. The difference between average returns for the smallest firms and the largest firms are all significant. Firm size seems to contain different information other than corporate liquidity, otherwise we should expect that size effect no longer exists after we control for the cash holding level. Panel B shows the average liquidity. Within a specific liquidity group, average firm cash holdings do not vary much with size. Panel C and Panel D display the average size and book-to-market ratios of the 25 portfolios. Panel E shows that the average leverage ratio declines with company size for each of the corporate liquidity group. Larger companies tend to borrow more than smaller firms regardless their cash position. Average leverage deviations from industry norm are shown in Panel G. For the most illiquid portfolios, all the five size portfolios tend to borrow more than industry average, and smaller firms tend to borrow more, while the most liquid portfolios tend to have lower than average leverage. Panel $\mathrm{H}$ displays the average cash flows for the 25 
subsequently sorted portfolios. For all 5 liquidity portfolios, big companies are able to provide more cash flows compared to small companies, given the corporate liquidity level. Panel I shows that cash flows generated by smaller companies are much riskier than large companies because these cash flows are more volatile. Furthermore, it is noticeable that the 5 most liquid portfolios have the most volatile cash flows compared to less liquid companies for all 5 size portfolios. Panel $\mathrm{J}$ examines the average beta coefficients. Though the pattern is not monotonic and obvious, it seems that larger firms tend to have lower betas than smaller firms within each liquidity group. Panel $\mathrm{K}$ through Panel $\mathrm{N}$ display the four different financial constraint measures for the 25 corporate liquidity-size portfolios. It can be seen that after the corporate liquidity is controlled for, larger companies seem to be less financially constrained than smaller companies, which is consistent with the literature.

\section{A.2. The Value Effect Controlled by Corporate Liquidity}

Table 2 presents the value effects when corporate liquidity is controlled for. Panel A shows there is a clear pattern that average rate of return increases with the book-tomarket ratios for each liquidity cohort. Value firms tend to offer higher returns regardless of the proportion of cash holding in total assets. The return differential between firms with highest and lowest book-to-market equity ratio is statistically significant. It seems that corporate liquidity contains different information other than book-to-market ratio, otherwise value effect would disappear after we remove the liquidity effect. According to Panel B, the differences of average corporate liquidity across BE/ME sub-portfolios are very small and can be neglected. For the most liquid portfolio, the average cash-to-asset ratio decreases monotonically as book-to-market equity increases. Panel C and Panel D display the average firm size and book-to-market value for the 25 corporate liquidity$\mathrm{BE} / \mathrm{ME}$ portfolios. Panel $\mathrm{F}$ shows that firms in the three bottom liquidity portfolios tend to hold less cash as a portion of their total assets compared to the industry average, but this negative deviation seems to shrink as the book-to-market equity increases. In other words, for relatively illiquid firms, value firms tend to deviate less negatively than growth firms in terms of cash holdings. However, this pattern reverses for the most liquid portfolio, where growth firms tend to deviate positively from the industry standard with a 
magnitude greater than value firms. Average leverage is displayed in Panel E, which shows how much growth firms tend to borrow more than value firms. In addition, Panel $\mathrm{G}$ provides evidence that compared to value firms, growth firms tend to raise more external debt than industry average. Panel $\mathrm{H}$ displays average cash flow for the 25 liquidity-book-to-market portfolios. There seems to be a concave relationship between cash flow and book-to-market ratio within each liquidity group. It is apparent that the growth firms tend to generate more volatile cash flow streams compared to value firms, as it can be seen from Panel I. While on average, liquid portfolios have higher cash flow volatilities than illiquid portfolios. Panel $J$ shows that there is an inverse relationship between book-to-market equity and average beta for each liquidity portfolio. In order to examine how the firms are subject to financial constraints, average total assets, $\mathrm{KZ}$ index and dividend payout are measure for each of the 25 portfolios. Panel K through panel N present the results. Value firms seem to be less financially constrained, although the relationship is not always monotonic.

\section{A.3. Additional Fama-MacBeth Two-Pass Regression}

When I estimate the Fama-MacBeth two-pass regression to test the effect of corporate liquidity on equity returns, in addition to the level of corporate liquidity, I also include the dummy variables that indicate the relevant liquidity position of companies. Liquidity constraint dummies are formed such that $\mathrm{D}_{-}$low equals one if the time series average of an individual firm's cash holdings falls below the bottom 30 percentile of the industry average level and zero otherwise. Excess liquidity dummy D_high equals one if the time series average of individual firm's cash holdings is higher than the top 30 percentile of the industry average level and zero otherwise. The results are presented in this part.

Panel A of Table 3 presents the results of models that include these dummy variables as well as interaction terms between liquidity dummy and corporate liquidity, in addition to firm betas derived from 25 portfolios in the first pass. Model 8 includes liquidity constraint dummy D_low as a regressor, whose coefficient is significantly positive. If a firm's liquid asset holding is lower than the bottom 30 percentile of its industry level, it is

considered to be liquidity constrained, and the monthly equity return of the firm will be 0.15 percent higher than those unconstrained firms. The interaction term of corporate 
liquidity and liquidity constraint dummy is added in model 9, which is positive and significant.

Model 10 and 11 show that neither excess liquidity dummy variable $D \_h i g h$ nor the interaction term of corporate liquidity and excess liquidity dummy has a significant effect on the stock returns. This indicates that holding excess cash does not necessarily hurt the firm, probably because the firm has accumulated abundant cash from successful operations, or maybe the firm piles up cash on purpose for the future growth opportunities. Furthermore, when both liquidity constraint dummy and excess liquidity dummy are included in the regression, the constraint dummy is still significantly positive but the excess liquidity dummy is insignificant.

The above estimations are repeated using the firm betas derived from the 27 portfolios, and the empirical results are exhibited in panel $\mathrm{B}$ of Table 3 . When the liquidity constraint dummy is added into the regression, neither the magnitude nor the significance of the corporate liquidity coefficient is strongly affected. Model 8 indicates that if a firm is liquidity constrained, the expected stock returns will increase by 0.183 percent per month compared to those unconstrained firms in the same industry. When the interaction term of corporate liquidity and liquidity constraint dummy is included in the regression in model 9, it turns out that the interaction term is significant. Model 10 examines how excess corporate liquidity can impact the stock returns. The coefficient of excess liquidity dummy is negative and but not significant. Model 11 includes the interaction term between corporate liquidity and the excess liquidity dummy. The negative coefficient on the interaction term indicates that if a firm keeps too much cash and other liquid assets compared to other firms in the same industry, its expected monthly stock return will be 0.253 percent lower on average. 


\section{REFERENCES}

Acharya, Viral V., Heitor Almeida, and Murillo Campello, 2006, Is Cash Negative Debt? A Hedging Perspective on Corporate Financial Policies, London Business School IFA Working Paper Series.

Almeida, Heitor, Murillo Campello, and Michael S. Weisbach, 2004, The Cash Flow Sensitivity of Cash, Journal of Finance 59, 1777-1804.

Anderson, Ronald, 2002, Capital Structure, Firm Liquidity and Growth, National Bank of Belgium working papers - Research Series.

Baker, Macolm, Richard S. Ruback, and Jeffrey Wurgler, 2004, Bahavioral Corporate Finance: A Survey, NBER working paper 10863, Handbook in Corporate Finance: Empirical Corporate Finance, edited by Espen Eckbo.

Banz, Rolf W., 1981, The Relationship Between Return and Market Value of Common Stocks, Journal of Financial Economics 9, 3-18.

Baskin, Jonathan B., 1987, Corporate Liquidity in Games of Monopoly Power, Review of Economics and Statistics 69, 312-319.

Basu, Sanjoy, 1977, Investment Performance of Common Stocks in Relation to Their Price-Earnings Ratios: A Test of the Efficient Market Hypothesis, Journal of Finance $32,663-682$.

Basu, Sanjoy, 1983, The Relationship Between Earnings' Yield, Market Value and Return For NYSE Common Stocks, Journal of Financial Economics 9, 129-56.

Baum, Christopher F., Mustafa Caglayan, Neslihan Ozkan, and Oleksandr Talavera, 2004, The Impact of Macroeconomic Uncertainty on Cash Holdings for Non-Financial Service Firms, Review of Financial Economics 15, 289-304.

Bhandari, Laxmi Chand, 1988, Debt/Equity Ratio and Expected Common Stock Returns: Empirical Evidence, Journal of Finance 43, 507-528.

Bodie, Zvi, Alex Kane, and Alan J. Marcus, 1999, Investments, $4^{\text {th }}$ edition, McGraw-Hill College.

Brennan, Michael J., and Avanidhar Subrahmanyam, 1996, Market Microstructure and Asset Pricing: On the Compensation for Illiquidity in Stock Returns, Journal of Financial Economics 41, 441-464.

Campbell, John Y., Jens Hilscher, and Jan Szilagyi, 2005, In Search of Distress Risk, Harvard Institute of Economic Research Discussion Paper No. 2081, Harvard University. 
Campello, Murillo, and Long Chen, 2005, Are Financial Constraints Priced? Evidence from Firm Fundamentals, Stocks, and Bonds, AFA 2006 Boston Meetings.

Chan, K.C., and Nai-fu Chen, 1991, Structural and Return Characteristics of Small and Large Firms, Journal of Finance 46, 1467-1484.

Chan, K.C., Nai-fu Chen, and David Hsieh, 1985, An exploratory Investigation of the Firm Size Effect, Journal of Financial Economics 14, 451-471.

Chordia, Tarun, Richard Roll, and Avanidhar Subrahmanyam, 2000, Commonality in Liquidity, Journal of Financial Economics 56, 3-28.

Chudson Walter A., 1945, The Pattern of Corporate Financial Structure: A Cross-Section View of Manufacturing, Mining, Trade, and Construction, 1937, National Bureau of Economic Research, Financial Research Program III: Studies in Business Financing, UMI, New York.

Cohen, Randolph B., Christopher Polk, and Tuomo Vuolteenaho, 2003, The Value Spread, Journal of Finance 58, 609-641.

Custodio, Claudia, Miguel A. Ferreira, and Clara C. Raposo, 2005, Cash Holdings and Business Conditions, working paper, SSRN.

DeAngelo, Harry, and Ronald W. Masulis, 1980, Optimal Capital Structure under Corporate and Personal Taxation, Journal of Financial Economics 8, 3-29.

Dittmar, Amy, and Jan Mahrt-Smith, 2007, Corporate Governance and the Value of Cash Holding, Journal of Financial Economics 83, 599-634.

Dittmar, Amy, Jan Mahrt-Smith, and Henri Servaes, 2003, International Corporate Governance and Corporate Cash Holdings, Journal of Financial and Quantitative Analysis, Special Edition on International Corporate Governance, 38, 111-134.

Faleye, Olububmi, 2004, Cash and Corporate Control, Journal of Finance 59, 2041-2060.

Fama, Eugene F., and Kenneth R. French, 1992, The Cross-section of Expected Stock Returns, Journal of Finance 47, 427-465.

Fama, Eugene F., and Kenneth R. French, 1993, Common Risk Factors in the Returns on Stocks and Bonds, Journal of Financial Economics 33, 3-56.

Fama, Eugene F., and Kenneth R. French, 1995, Size and Book-to-Market Factors in Earnings and Returns, Journal of Finance 50, 131-155.

Fama, Eugene F., and Kenneth R. French, 1996, Multifactor Explanations of Asset Pricing Anomalies, Journal of Finance 51, 55-84. 
Fama, Eugene F., and James MacBeth, 1973, Risk, Return and Equilibrium: Empirical Tests, Journal of Political Economy 81, 607-636.

Faulkender, Michael, 2004, Cash Holdings among Small Business, working paper, Washington University.

Faulkender, Michael, and Rong Wang, 2006, Corporate Financial Policy and the Value of Cash, Journal of Finance 61, 1957-1990.

Fazzari, Steven, R., Glenn Hubbard, and Bruce Petersen, 1988, Financing Constraints and Corporate Investment, Brooking Papers on Economic Activity 1, 141-195.

Fischer, Edwin. O., Robert Heinkel, and Josef Zechner, 1989, Optimal Dynamic Capital Structure Choice: Theory and Tests, Journal of Finance 44, 19-40.

Harford, Jarrad. 1999, Corporate Cash Reserves and Acquisitions, Journal of Finance 54, 1969-1997.

Hovakimian, Armen, Tim Opler, and Sheridan Titman, 2001, The Debt-equity choice, Journal of Financial and Quantitative Analysis 36, 1-24.

Hovakimian, Armen, Gayne Hovakimian, and Hasan Tehranian, 2004, Determinants of Target Capital Structure: the Case of Dual Debt and Equity Issues, Journal of Financial Economics 71, 349-380.

Kim, Chang-Soo, David C. Mauer, and Ann E. Sherman, 1998, The Determinants of Corporate Liquidity: Theory and Evidence, Journal of Financial and Quantitative Analysis 33, 335-359.

Kothari, S. P., and Jay Shanken, 1997, Book-to-Market, Dividend Yield, and Expected Market Returns: A Time-series Analysis, Journal of Financial Economics 44, 169203.

Lakonishok, Josef, Andrei Shleifer, and Rovert W. Vishny, 1994, Contrarian Investment, Extrapolation, and Risk, Journal of Finance 49, 1541-1578.

Lamont, Owen, Christopher Polk, and Jesús Saá-Requejo, 2001, Financial Constraints and Stock Returns, The Review of Financial Studies 14, 529-554.

Lipson, Marc, and Sandra Mortal, 2004, Capital Structure Decisions and Equity Market Liquidity, Working paper, SSRN.

Mikkelson, Wayne, and Megan Partch, 2003, Do Persistent Large Cash Reserve Hinder Performance?, Journal of Financial and Quantitative Analysis 38, 275-294. 
Myers, Stewart C., 1977, Determinants of Corporate Borrowing, Journal of Financial Economics 5, 147-175.

Opler, Tim, Lee Pinkowitz, Rene Stulz, and Rohan Williamson, 1999, The Determinants and Implications of Corporate Cash Holdings, Journal of Financial Economics 52, 346.

Pástor, Luboš, and Stambaugh, Robert F., 2003, Liquidity Risk and Expected Stock Returns. Journal of Political Economy 111, 642-685.

Pinkowitz, Lee, and Rohan Williamson, 2004, What is a Dollar Worth? The Market Value of Cash Holdings, working paper, Georgetown University.

Pinkowitz, Lee, and Rohan Williamson, 2001, Bank Power and Cash Holdings: Evidence from Japan, Review of Financial Studies 14, 1059-1082.

Pontiff, Jeffrey, and Lawrence D. Schall, 1998, Book-to-Market Ratios as Predictors of Market Returns, Journal of Financial Economics 49, 141-160.

Reinganum, Marc R., 1981, A New Empirical Perspective on the CAPM, Journal of Financial and Quantitative Analysis 16, 439-462.

Rosenberg, Barr, Kenneth Reid, and Ronald Lanstein, 1985, Persuasive Evidence of Market Inefficiency, Journal of Portfolio Management 11, 9-17.

Ross, Stephen A., 1976a, The Arbitrage Theory of Capital Asset Pricing, Journal of Economic Theory 13, 341-360

Ross, Stephen A., 1976b, Risk, Return, and Arbitrage, In Risk and Return in Finance, Edited by Irwin Friend and James Bicksler, 189-218. Cambridge, MA.: Ballinger.

Schultz, Paul, 1983, Transaction Costs and the Small Firm Effect: A comment, Journal of Financial Economics 12, 81-88.

Sibilkov, Valeriy, 2005, Is Cash More Valuable for Constrained Firms? working paper, Purdue University.

Stoll, Hans R., and Robert E. Whaley, 1983, Transaction Costs and the Small Firm Effect, Journal of Financial Economics 12, 57-79.

Vassalou, Maria, and Yuhang Xing, 2004, Default Risk in Equity Returns, Journal of Finance 59, 831-868.

Vogel, Robert C., and G. S. Maddala, 1967, Cross-Sectional Estimates of Liquid Asset Demand by Manufacturing Corporations, Journal of Finance 22, 557-575. 
Whited, Toni M., 1992, Debt, Liquidity Constraints and Corporate Investment: Evidence from Panel Data, Journal of Finance 47, 425-460.

Whited, Toni M., and Guojun Wu, 2006, Financial Constraints Risk, Review of Financial Studies 19, 531-559. 
Table 1. Summary Statistics for the 1962.1 - 2005.12 Sample

Stocks are sorted into quintiles on the basis of monthly stock return, size, BE/ME, CL, Leverage, CL deviation and Leverage deviation. Sample mean and standard deviation, and average of each quintile are displayed. Return is monthly stock returns in percentage. SIZE denotes the market value of the stocks, measured in June of each year. BE/ME denotes the book-to-market equity. CL is corporate liquidity, measured as the ratio of cash and marketable securities to total assets. Leverage is the debtto-asset ratio. In order to remove the effect of industry differentials, CL deviation and Leverage deviation denote the deviation of firms' cash holdings and financial leverage compared to the industry average based on the Fama-French 17 industries. Financial constraint measures include total assets, $\mathrm{KZ}$ index and dividend payout. Total assets is the book value of total assets. $\mathrm{KZ}$ index is a linear index of firm financial constraints constructed based on Kaplan and Zingales (1997). Dividend payout is calculated as the ratio of total dividends distributions to operating income. Distribution to Shareholders is defined as the sum of total dividends and stock repurchases divided by operating income.

\begin{tabular}{lrrrrrrr}
\hline Variable & Mean & $1^{\text {st }}$ & $2^{\text {nd }}$ & $3^{\text {rd }}$ & $4^{\text {th }}$ & $5^{\text {th }}$ & Std. Dev. \\
\hline Return & 1.143 & -18.809 & -5.811 & 0.068 & 6.350 & 23.920 & 16.289 \\
SIZE & 837.380 & 10.294 & 35.743 & 97.215 & 307.910 & 3735.700 & 3209.500 \\
BE/ME & 1.158 & 0.151 & 0.377 & 0.593 & 0.907 & 3.762 & 4.020 \\
CL & 0.158 & 0.010 & 0.035 & 0.078 & 0.175 & 0.491 & 0.195 \\
Leverage & 0.418 & 0.128 & 0.291 & 0.411 & 0.523 & 0.739 & 0.222 \\
CL & 0 & -0.194 & -0.082 & -0.039 & 0.031 & 0.281 & 0.175 \\
deviation & & & & & & & \\
Leverage & 0 & -0.309 & -0.132 & -0.019 & 0.091 & 0.307 & 0.232 \\
deviation & & & & & & & \\
Cash Flow & 0.018 & -0.284 & 0.032 & 0.071 & 0.103 & 0.168 & 0.234 \\
Cash Flow & 0.220 & 0.024 & 0.050 & 0.086 & 0.163 & 0.779 & 0.504 \\
$\begin{array}{l}\text { Volatility } \\
\text { Total Assets }\end{array}$ & 1267.141 & 15.865 & 52.879 & 138.170 & 422.950 & 5704.920 & 5069.896 \\
KZ index & -2.411 & -16.902 & -3.699 & 0.368 & 1.516 & 6.663 & 13.827 \\
$\begin{array}{l}\text { Dividend } \\
\text { Payout }\end{array}$ & 0.101 & -0.108 & 0.000 & 0.016 & 0.137 & 0.461 & 0.344 \\
$\begin{array}{l}\text { Distribution } \\
\text { to }\end{array}$ & & & & & & & \\
Shareholders & 0.177 & -0.244 & 0.000 & 0.059 & 0.206 & 0.862 & 0.712 \\
\hline
\end{tabular}




\section{Table 2. Firm Characteristics and Corporate Liquidity}

SIZE denotes the market value of the stocks, measured in June of each year. BE/ME denotes the book-to-market equity. CL is the corporate liquidity, measured as the ratio of cash and marketable securities to total assets. Leverage is debt-to-asset ratio. In order to remove the effect of industry differentials, CL deviation and Leverage deviation denote the deviation of firms' cash holdings and financial leverage compared to the industry average based on the Fama-French 17 industries. Panel A shows the overall correlation between firms' characteristics. Panel B summarizes the time series average of the cross-sectional correlations between firms' features. Panel C shows the cross sectional average time-series correlations between firm characteristics

Panel A. Overall Correlation between Average Firm Characteristics

\begin{tabular}{|c|c|c|c|c|c|c|c|c|c|c|c|}
\hline & SIZE & $\mathrm{BE} / \mathrm{ME}$ & $\mathrm{CL}$ & Leverage & $\begin{array}{c}\mathrm{CL} \\
\text { deviation }\end{array}$ & $\begin{array}{l}\text { Leverage } \\
\text { deviation }\end{array}$ & $\begin{array}{l}\text { Cash } \\
\text { Flow }\end{array}$ & $\begin{array}{c}\text { Total } \\
\text { Assets }\end{array}$ & $\mathrm{KZ}$ index & $\begin{array}{l}\text { Dividend } \\
\text { Payout }\end{array}$ & $\begin{array}{l}\text { Distribution } \\
\text { to } \\
\text { Shareholders }\end{array}$ \\
\hline SIZE & 1 & -0.0043 & 0.0123 & -0.0013 & -0.0394 & -0.0020 & 0.0438 & 0.5594 & -0.0315 & 0.0371 & 0.0480 \\
\hline $\mathrm{BE} / \mathrm{ME}$ & & 1 & -0.0039 & -0.0002 & -0.0021 & -0.0001 & 0.0268 & 0.2649 & -0.0376 & 0.0360 & 0.0137 \\
\hline $\mathrm{CL}$ & & & 1 & -0.3065 & 0.9032 & -0.3300 & 0.2863 & -0.0762 & 0.0655 & -0.0833 & -0.0449 \\
\hline Leverage & & & & 1 & -0.2831 & 0.9995 & -0.1212 & 0.0475 & 0.0888 & -0.0322 & -0.0185 \\
\hline CL deviation & & & & & 1 & -0.0331 & 0.1936 & -0.0877 & 0.0215 & -0.0426 & -0.0389 \\
\hline $\begin{array}{l}\text { Leverage } \\
\text { deviation }\end{array}$ & & & & & & 1 & -0.1486 & 0.0009 & 0.1290 & -0.0901 & -0.0496 \\
\hline Cash Flow & & & & & & & 1 & 0.0497 & -0.1091 & 0.0723 & 0.0748 \\
\hline Total Assets & & & & & & & & 1 & -0.0682 & 0.0727 & 0.0606 \\
\hline $\mathrm{KZ}$ index & & & & & & & & & 1 & -0.9798 & -0.6792 \\
\hline $\begin{array}{l}\text { Dividend } \\
\text { Payout }\end{array}$ & & & & & & & & & & 1 & 0.6918 \\
\hline $\begin{array}{l}\text { Distribution to } \\
\text { Shareholders }\end{array}$ & & & & & & & & & & & 1 \\
\hline
\end{tabular}


Table 2. (continued)

Panel B. Time Series Average of Cross-sectional Correlations between Firm Characteristics

\begin{tabular}{|c|c|c|c|c|c|c|c|c|c|c|c|}
\hline & SIZE & $\mathrm{BE} / \mathrm{ME}$ & $\mathrm{CL}$ & Leverage & $\begin{array}{c}\mathrm{CL} \\
\text { deviation }\end{array}$ & $\begin{array}{l}\text { Leverage } \\
\text { deviation }\end{array}$ & $\begin{array}{l}\text { Cash } \\
\text { Flow }\end{array}$ & $\begin{array}{c}\text { Total } \\
\text { Assets }\end{array}$ & $\mathrm{KZ}$ index & $\begin{array}{c}\text { Dividend } \\
\text { Payout }\end{array}$ & $\begin{array}{l}\text { Distribution } \\
\text { to } \\
\text { Shareholders }\end{array}$ \\
\hline SIZE & 1 & -0.5160 & 0.0475 & -0.0513 & -0.0076 & -0.0498 & 0.1146 & 0.6706 & -0.0746 & 0.0820 & 0.0652 \\
\hline $\mathrm{BE} / \mathrm{ME}$ & & 1 & -0.0503 & 0.0360 & -0.0668 & 0.0321 & -0.0945 & 0.2191 & -0.0425 & 0.0337 & 0.0172 \\
\hline CL & & & 1 & -0.2870 & 0.9637 & -0.2909 & 0.0145 & -0.0743 & 0.0191 & -0.0028 & 0.0106 \\
\hline Leverage & & & & 1 & -0.2963 & 0.9820 & -0.2863 & 0.0119 & 0.1587 & -0.1033 & -0.0890 \\
\hline CL deviation & & & & & 1 & -0.3018 & 0.0285 & -0.0577 & -0.0251 & 0.0029 & 0.0114 \\
\hline $\begin{array}{l}\text { Leverage } \\
\text { deviation }\end{array}$ & & & & & & 1 & -0.2860 & 0.0130 & 0.1527 & -0.1043 & -0.0876 \\
\hline Cash Flow & & & & & & & 1 & 0.0599 & -0.0321 & 0.0118 & 0.0196 \\
\hline Total Assets & & & & & & & & 1 & -0.0124 & 0.1102 & 0.0777 \\
\hline KZ index & & & & & & & & & 1 & -0.9740 & -0.7732 \\
\hline Dividend Payout & & & & & & & & & & 1 & 0.7939 \\
\hline Distribution to & & & & & & & & & & & 1 \\
\hline Shareholders & & & & & & & & & & & 1 \\
\hline
\end{tabular}

Panel C. Cross Sectional Average of Time-series Correlations between Firm Characteristics

\begin{tabular}{|c|c|c|c|c|c|c|c|c|c|c|c|}
\hline & SIZE & $\mathrm{BE} / \mathrm{ME}$ & $\mathrm{CL}$ & Leverage & $\begin{array}{c}\mathrm{CL} \\
\text { deviation }\end{array}$ & $\begin{array}{l}\text { Leverage } \\
\text { deviation }\end{array}$ & $\begin{array}{l}\text { Cash } \\
\text { Flow }\end{array}$ & $\begin{array}{c}\text { Total } \\
\text { Assets }\end{array}$ & $\mathrm{KZ}$ index & $\begin{array}{c}\text { Dividend } \\
\text { Payout }\end{array}$ & $\begin{array}{l}\text { Distribution } \\
\text { to } \\
\text { Shareholders }\end{array}$ \\
\hline SIZE & 1 & -0.4850 & 0.0529 & -0.1635 & 0.0197 & -0.1159 & 0.1650 & 0.4765 & 0.0124 & 0.0645 & 0.0817 \\
\hline $\mathrm{BE} / \mathrm{ME}$ & & 1 & -0.0303 & -0.1192 & -0.0149 & -0.1268 & -0.1033 & 0.1161 & -0.1938 & -0.0899 & -0.0085 \\
\hline CL & & & 1 & -0.2761 & 0.8480 & -0.2241 & 0.1227 & -0.0965 & 0.1430 & -0.0484 & -0.0106 \\
\hline Leverage & & & & 1 & -0.2482 & 0.8285 & -0.2984 & 0.0427 & 0.3881 & 0.0918 & -0.0311 \\
\hline CL deviation & & & & & 1 & -0.2742 & 0.1046 & -0.1630 & -0.1404 & -0.0563 & -0.0215 \\
\hline $\begin{array}{l}\text { Leverage } \\
\text { deviation }\end{array}$ & & & & & & 1 & -0.1785 & 0.0149 & 0.0741 & -0.0459 & -0.0667 \\
\hline Cash Flow & & & & & & & 1 & 0.0102 & -0.0877 & -0.1283 & -0.0072 \\
\hline Total Assets & & & & & & & & 1 & -0.0654 & 0.0423 & 0.0839 \\
\hline $\mathrm{KZ}$ index & & & & & & & & & 1 & -0.5231 & -0.5142 \\
\hline Dividend Payout & & & & & & & & & & 1 & 0.7180 \\
\hline Distribution to & & & & & & & & & & & 1 \\
\hline Shareholders & & & & & & & & & & & 1 \\
\hline
\end{tabular}




\section{Table 3. Quantile Average Sorted by Firm Characteristics}

From 1962.1 to 2005.12 , at the beginning of each month, stocks are sorted into 5 portfolios on the basis of corporate liquidity, size and BE/ME. The equally weighted averages of return, corporate liquidity, size and $\mathrm{BE} / \mathrm{ME}$ are reported. "Liquid-Illiquid" is the differences in return, corporate liquidity, size and BE/ME between the most liquid and illiquid portfolios. "Small-Big" is the differences in return, corporate liquidity, size and BE/ME between the smallest and biggest size portfolios. "High-Low" is the differences in return, corporate liquidity, size and $\mathrm{BE} / \mathrm{ME}$ between the highest $\mathrm{BE} / \mathrm{ME}$ and lowest $\mathrm{BE} / \mathrm{ME}$ portfolios.

Panel A. Portfolios Sorted on the Basis of Corporate Liquidity

\begin{tabular}{lrrrrrrr}
\hline & Illiquid 1 & 2 & 3 & 4 & Liquid 5 & Liquid - Illiquid & t-statistic \\
\hline Return* & 0.731 & 0.961 & 1.283 & 1.706 & 2.000 & 1.269 & 22.105 \\
$C L^{* *}$ & 0.010 & 0.035 & 0.078 & 0.175 & 0.495 & 0.485 & \\
Size** $_{\text {BE/ME }}$ & 1095.100 & 1141.600 & 1250.500 & 1276.700 & 952.710 & -142.390 & \\
Leverage $_{\mathrm{CF}^{\dagger}}$ & 1.498 & 1.750 & 3.094 & 3.949 & 1.695 & 0.197 & -0.220 \\
$\mathrm{CF}_{\text {S sigma }}^{\dagger \dagger}$ & 0.554 & 0.493 & 0.451 & 0.382 & 0.334 & -0.166 & \\
Total Assets & 0.005 & 0.036 & 0.041 & 0.023 & -0.161 & 0.335 & \\
KZ index & 0.158 & 0.224 & 0.264 & 0.368 & 0.493 & -907.85 & \\
DivPayout & 1373.67 & 1281.92 & 1530.84 & 1268.73 & 465.82 & -0.058 & \\
Dist. to Shareholder & -2.228 & -3.039 & -3.285 & -2.781 & -0.722 & -0.063 & \\
\hline
\end{tabular}

Panel B. Portfolios Sorted on the Basis of Firm Size

\begin{tabular}{|c|c|c|c|c|c|c|c|}
\hline & Small 1 & 2 & 3 & 4 & Big 5 & Small-Big & t-statistic \\
\hline Return* & 2.212 & 2.165 & 1.562 & 1.078 & -0.335 & 2.547 & 48.561 \\
\hline$C L * *$ & 0.130 & 0.157 & 0.182 & 0.185 & 0.139 & 0.008 & \\
\hline Size*** & 10.250 & 35.743 & 97.215 & 307.910 & 5265.500 & 5255.250 & \\
\hline $\mathrm{BE} / \mathrm{ME}$ & 6.968 & 1.963 & 1.373 & 1.061 & 0.623 & -6.345 & \\
\hline Leverage & 0.580 & 0.445 & 0.403 & 0.383 & 0.403 & -0.178 & \\
\hline $\mathrm{CF}^{\dagger}$ & -0.154 & -0.027 & 0.003 & 0.041 & 0.081 & 0.234 & \\
\hline $\mathrm{CF}$ sigma $^{\dagger \dagger}$ & 0.436 & 0.345 & 0.303 & 0.253 & 0.171 & -0.264 & \\
\hline Total Assets & 112.12 & 204.13 & 354.99 & 755.05 & 4494.70 & -4382.58 & \\
\hline $\mathrm{KZ}$ index & -0.407 & -1.517 & -2.260 & -3.294 & -4.576 & 4.169 & \\
\hline DivPayout & 0.049 & 0.077 & 0.094 & 0.120 & 0.157 & -0.107 & \\
\hline Dist. to Shareholder & 0.084 & 0.129 & 0.159 & 0.198 & 0.308 & -0.224 & \\
\hline
\end{tabular}

Panel C. Portfolios Sorted on the Basis of BE/ME

\begin{tabular}{lrrrrrrr}
\hline & Low 1 & 2 & 3 & 4 & High 5 & High - Low & t-statistic \\
\hline Return* & -0.835 & 0.509 & 1.328 & 2.278 & 3.401 & 4.236 & 72.522 \\
$C L^{* *}$ & 0.238 & 0.187 & 0.142 & 0.121 & 0.105 & -0.133 & \\
Size*** & 2730.700 & 1556.200 & 782.680 & 438.830 & 208.150 & -2522.550 & \\
BE/ME & 0.193 & 0.357 & 0.576 & 0.891 & 10.355 & 10.162 & \\
Leverage & 0.607 & 0.382 & 0.394 & 0.405 & 0.426 & -0.181 & \\
CF $^{\dagger}$ & -0.241 & 0.045 & 0.055 & 0.049 & 0.035 & 0.276 & \\
$\mathrm{CF}_{\text {sigma }}^{\dagger \dagger}$ & 0.650 & 0.297 & 0.223 & 0.179 & 0.158 & -0.492 & \\
Total Assets & 1245.32 & 1192.49 & 994.68 & 804.64 & 1684.42 & 439.10 & -3.124 \\
KZ index & -0.007 & -2.362 & -3.102 & -3.452 & -3.131 & 0.049 & \\
DivPayout & 0.061 & 0.095 & 0.112 & 0.119 & 0.109 & 0.013 & \\
Dist. to Shareholder & 0.146 & 0.190 & 0.191 & 0.192 & 0.159 & & \\
\hline
\end{tabular}

*Average return is reported in per month percentage terms. ${ }^{* *} C L$ stands for corporate liquidity, which is measured by the proportion of cash and cash equivalents in the firm's total assets. ***Firms size is in millions of dollars. ${ }^{\dagger} \mathrm{CF}$ is cash flow. ${ }^{\# C} \mathrm{CF}$ _sigma is the cash flow volatility. 


\section{Table 4. Corporate Liquidity Effect Controlled by Size}

From 1985.7 to 2003.12, at the beginning of each month, stocks are sorted into 5 size portfolios. Within each size portfolios, stocks are subsequently sorted into 5 portfolios on the basis of corporate liquidity. The equally weighted averages of return, corporate liquidity, size, BE/ME, leverage, deviations of corporate liquidity and leverage from industry norm, beta and alternative financial constraint measures are reported. "Liquid-Illiquid" is the differences in the returns between the most liquid and illiquid portfolios.

Panel A. Average Return*

\begin{tabular}{lrrrrrrr}
\hline & Illiquid 1 & 2 & 3 & 4 & Liquid 5 & Liquid - Illiquid & t-statistic \\
\hline Small 1 & 1.671 & 1.936 & 2.287 & 2.752 & 3.162 & 1.492 & 2.631 \\
2 & 0.689 & 0.976 & 1.061 & 1.617 & 1.858 & 1.169 & 2.418 \\
3 & 0.690 & 0.867 & 1.066 & 1.343 & 1.511 & 0.820 & 1.771 \\
4 & 0.791 & 0.815 & 1.184 & 1.255 & 1.382 & 0.590 & 1.362 \\
Big 5 & 0.779 & 0.785 & 0.986 & 1.029 & 1.316 & 0.537 & 1.418 \\
\hline
\end{tabular}

* Average return is monthly stock returns measured in percentage terms

Panel B. Average Corporate Liquidity

\begin{tabular}{lrrrrr}
\hline & Illiquid 1 & 2 & 3 & 4 & Liquid 5 \\
\hline Small 1 & 0.014 & 0.036 & 0.074 & 0.151 & 0.374 \\
2 & 0.016 & 0.042 & 0.090 & 0.182 & 0.403 \\
3 & 0.017 & 0.044 & 0.095 & 0.189 & 0.408 \\
4 & 0.016 & 0.041 & 0.085 & 0.168 & 0.379 \\
Big 5 & 0.015 & 0.036 & 0.069 & 0.130 & 0.297 \\
\hline
\end{tabular}

Panel C. Average Size**

\begin{tabular}{lrrrrr}
\hline & Illiquid 1 & 2 & 3 & 4 & Liquid 5 \\
\hline Small 1 & 12.001 & 12.149 & 12.272 & 12.603 & 12.926 \\
2 & 39.919 & 40.208 & 40.189 & 40.853 & 40.870 \\
3 & 103.680 & 104.050 & 104.220 & 103.970 & 103.100 \\
4 & 299.880 & 299.350 & 300.850 & 298.050 & 290.260 \\
Big 5 & 2957.000 & 4195.300 & 4133.100 & 4683.900 & 3694.800 \\
\hline
\end{tabular}

**Firms size is in millions of dollars

Panel D. Average BE/ME

\begin{tabular}{lrrrrr}
\hline & Illiquid 1 & 2 & 3 & 4 & Liquid 5 \\
\hline Small 1 & 3.083 & 3.021 & 6.521 & 7.659 & 2.390 \\
2 & 1.104 & 1.438 & 2.078 & 2.115 & 0.798 \\
3 & 0.986 & 1.258 & 1.349 & 1.558 & 1.048 \\
4 & 0.828 & 0.984 & 1.251 & 1.136 & 0.630 \\
Big 5 & 0.660 & 0.637 & 0.716 & 0.678 & 0.478 \\
\hline
\end{tabular}


Table 4. (continued)

Panel E. Average Leverage Ratio

\begin{tabular}{lrrrrr}
\hline & Illiquid 1 & 2 & 3 & 4 & Liquid 5 \\
\hline Small 1 & 0.706 & 0.551 & 0.522 & 0.469 & 0.491 \\
2 & 0.525 & 0.496 & 0.465 & 0.400 & 0.305 \\
3 & 0.487 & 0.476 & 0.430 & 0.379 & 0.296 \\
4 & 0.462 & 0.450 & 0.414 & 0.369 & 0.295 \\
Big 5 & 0.447 & 0.437 & 0.401 & 0.370 & 0.314 \\
\hline
\end{tabular}

Panel F. Average Cash Holding Deviation from Industry Norm

\begin{tabular}{lrrrrr}
\hline & Illiquid 1 & 2 & 3 & 4 & Liquid 5 \\
\hline Small 1 & -0.112 & -0.093 & -0.060 & 0.010 & 0.222 \\
2 & -0.106 & -0.085 & -0.044 & 0.037 & 0.244 \\
3 & -0.102 & -0.081 & -0.037 & 0.046 & 0.247 \\
4 & -0.101 & -0.083 & -0.043 & 0.028 & 0.220 \\
Big 5 & -0.102 & -0.085 & -0.056 & -0.007 & 0.143 \\
\hline
\end{tabular}

Panel G. Average Leverage Deviation from Industry Norm

\begin{tabular}{lrrrrr}
\hline & Illiquid 1 & 2 & 3 & 4 & Liquid 5 \\
\hline Small 1 & 0.228 & 0.117 & 0.084 & 0.033 & -0.095 \\
2 & 0.086 & 0.062 & 0.033 & -0.031 & -0.122 \\
3 & 0.054 & 0.043 & -0.004 & -0.053 & -0.128 \\
4 & 0.029 & 0.017 & -0.019 & -0.058 & -0.125 \\
Big 5 & 0.014 & 0.006 & -0.027 & -0.051 & -0.101 \\
\hline
\end{tabular}

Panel H. Average Cash Flow

\begin{tabular}{lrrrrr}
\hline & Illiquid 1 & 2 & 3 & 4 & Liquid 5 \\
\hline Small 1 & -0.124 & -0.019 & -0.032 & -0.077 & -0.228 \\
2 & 0.037 & 0.035 & 0.024 & 0.013 & -0.050 \\
3 & 0.062 & 0.045 & 0.055 & 0.047 & 0.001 \\
4 & 0.078 & 0.078 & 0.080 & 0.079 & 0.060 \\
Big 5 & 0.087 & 0.088 & 0.092 & 0.100 & 0.113 \\
\hline
\end{tabular}

Panel I. Average Cash Flow Volatility

\begin{tabular}{lrrrrrrr}
\hline & Illiquid 1 & 2 & 3 & 4 & Liquid 5 & Liquid - Illiquid & t-statistic \\
\hline Small 1 & 0.372 & 0.297 & 0.338 & 0.370 & 0.878 & 0.506 & 13.002 \\
2 & 0.302 & 0.308 & 0.347 & 0.331 & 0.615 & 0.313 & 11.286 \\
3 & 0.237 & 0.233 & 0.271 & 0.388 & 0.515 & 0.278 & 8.772 \\
4 & 0.206 & 0.245 & 0.267 & 0.334 & 0.574 & 0.368 & 12.922 \\
Big 5 & 0.232 & 0.234 & 0.225 & 0.349 & 0.511 & 0.279 & 9.386 \\
\hline
\end{tabular}


Table 4. (continued)

Panel J. Average Beta

\begin{tabular}{lrrrrrrr}
\hline & Illiquid 1 & 2 & 3 & 4 & Liquid 5 & Liquid - Illiquid & t-statistic \\
\hline Small 1 & 0.934 & 1.051 & 1.071 & 1.294 & 1.352 & 0.418 & 12.297 \\
2 & 0.929 & 0.948 & 1.199 & 1.239 & 1.412 & 0.483 & 16.805 \\
3 & 0.957 & 0.990 & 1.062 & 1.341 & 1.447 & 0.490 & 18.594 \\
4 & 0.855 & 0.907 & 0.998 & 1.299 & 1.419 & 0.564 & 17.985 \\
Big 5 & 0.850 & 0.868 & 0.896 & 1.138 & 1.289 & 0.439 & 15.012 \\
\hline
\end{tabular}

F-stat for Beta difference of all Liquidity-size quintiles: $F$-value $=57.37 ; p$ value $=2.7622 \mathrm{e}-009$

F-stat for Beta difference of most Liquid and illiquid-size quintiles: $F$-value $=92.16 ; p$ value $=0.0007$

Panel K. Average Total Assets

\begin{tabular}{lrrrrrrr}
\hline & Illiquid 1 & 2 & 3 & 4 & Liquid 5 & Liquid - Illiquid & t-statistic \\
\hline Small 1 & 62.003 & 112.361 & 227.382 & 172.031 & 39.614 & -22.389 & -6.201 \\
2 & 133.610 & 220.264 & 318.583 & 165.832 & 58.101 & -75.509 & -13.661 \\
3 & 276.611 & 454.123 & 429.181 & 379.054 & 189.732 & -86.87 & -6.939 \\
4 & 672.692 & 969.914 & 1043.428 & 669.625 & 290.593 & -382.1 & -14.930 \\
Big 5 & 3874.335 & 4722.146 & 4472.523 & 3892.813 & 1948.212 & -1926.1 & -14.759 \\
\hline
\end{tabular}

Panel L. Average KZ index

\begin{tabular}{lrrrrrrr}
\hline & Illiquid 1 & 2 & 3 & 4 & Liquid 5 & Liquid - Illiquid & t-statistic \\
\hline Small 1 & 0.261 & -0.863 & -0.368 & -0.910 & -1.989 & -2.251 & -12.767 \\
2 & -2.339 & -2.408 & -1.911 & -2.316 & -2.684 & -0.345 & -1.644 \\
3 & -3.432 & -3.712 & -3.619 & -3.412 & -4.002 & -0.570 & -2.230 \\
4 & -4.581 & -4.456 & -4.982 & -4.465 & -4.873 & -0.292 & -1.253 \\
Big 5 & -6.765 & -6.581 & -6.964 & -6.708 & -5.361 & 1.404 & 6.889 \\
\hline
\end{tabular}

Panel M. Average DivPayout

\begin{tabular}{lrrrrrrr}
\hline & Illiquid 1 & 2 & 3 & 4 & Liquid 5 & Liquid - Illiquid & t-statistic \\
\hline Small 1 & 0.045 & 0.056 & 0.054 & 0.055 & 0.072 & 0.026 & 6.243 \\
2 & 0.096 & 0.097 & 0.089 & 0.088 & 0.087 & -0.008 & -1.883 \\
3 & 0.126 & 0.133 & 0.128 & 0.120 & 0.116 & -0.011 & -1.937 \\
4 & 0.154 & 0.152 & 0.167 & 0.145 & 0.149 & -0.005 & -0.904 \\
Big 5 & 0.211 & 0.211 & 0.217 & 0.208 & 0.171 & -0.040 & -7.948 \\
\hline
\end{tabular}

Panel N. Average Distribution to Shareholders

\begin{tabular}{lrrrrrrr}
\hline & Illiquid 1 & 2 & 3 & 4 & Liquid 5 & Liquid - Illiquid & t-statistic \\
\hline Small 1 & 0.068 & 0.094 & 0.092 & 0.085 & 0.100 & 0.031 & 5.428 \\
2 & 0.141 & 0.160 & 0.136 & 0.149 & 0.123 & -0.018 & -2.983 \\
3 & 0.177 & 0.191 & 0.187 & 0.184 & 0.164 & -0.012 & -2.109 \\
4 & 0.210 & 0.207 & 0.241 & 0.209 & 0.212 & 0.002 & 0.286 \\
Big 5 & 0.299 & 0.302 & 0.323 & 0.308 & 0.269 & -0.030 & -4.763 \\
\hline
\end{tabular}




\section{Table 5. Corporate Liquidity Effect Controlled by BE/ME}

From 1962.1 to 2005.12 , at the beginning of each month, stocks are sorted into $5 \mathrm{BE} / \mathrm{ME}$ portfolios. Within each size portfolios, stocks are subsequently sorted into 5 portfolios on the basis of corporate liquidity. The equally weighted averages of return, corporate liquidity, size, BE/ME, leverage, deviations of corporate liquidity and leverage from industry norm, beta and alternative financial constraint measures are reported. "Liquid-Illiquid" is the differences in the returns between the most liquid and illiquid portfolios.

Panel A. Average Return*

\begin{tabular}{lrrrrrrr}
\hline & Illiquid 1 & 2 & 3 & 4 & Liquid 5 & Liquid - Illiquid & t-statistic \\
\hline Low 1 & -0.352 & -0.068 & 0.313 & 0.366 & 0.707 & 1.059 & 2.274 \\
2 & 0.263 & 0.470 & 0.756 & 1.245 & 1.862 & 1.599 & 3.506 \\
3 & 0.703 & 0.898 & 1.132 & 1.541 & 2.209 & 1.505 & 3.418 \\
4 & 1.135 & 1.322 & 1.658 & 1.993 & 2.634 & 1.499 & 3.429 \\
High 5 & 2.236 & 2.435 & 2.678 & 2.959 & 3.886 & 1.650 & 3.494 \\
\hline
\end{tabular}

* Average return is monthly stock returns measured in percentage terms

Panel B. Average Corporate Liquidity

\begin{tabular}{lrrrrr}
\hline & Illiquid 1 & 2 & 3 & 4 & Liquid 5 \\
\hline Low 1 & 0.023 & 0.071 & 0.149 & 0.264 & 0.483 \\
2 & 0.017 & 0.046 & 0.097 & 0.187 & 0.398 \\
3 & 0.015 & 0.037 & 0.073 & 0.145 & 0.338 \\
4 & 0.015 & 0.033 & 0.064 & 0.127 & 0.314 \\
High 5 & 0.014 & 0.031 & 0.059 & 0.110 & 0.283 \\
\hline
\end{tabular}

Panel C. Average Size**

\begin{tabular}{lrrrrr}
\hline & Illiquid 1 & 2 & 3 & 4 & Liquid 5 \\
\hline Low 1 & 2514.400 & 2974.600 & 2251.900 & 1646.800 & 1086.500 \\
2 & 1525.700 & 1652.500 & 1306.100 & 809.160 & 475.580 \\
3 & 711.250 & 962.610 & 834.770 & 536.650 & 256.770 \\
4 & 458.050 & 533.580 & 551.260 & 410.630 & 180.480 \\
High 5 & 246.300 & 230.910 & 264.000 & 248.800 & 119.990 \\
\hline
\end{tabular}

**Firms size is in millions of dollars

Panel D. Average BE/ME

\begin{tabular}{lrrrrr}
\hline & Illiquid 1 & 2 & 3 & 4 & Liquid 5 \\
\hline Low 1 & 0.269 & 0.271 & 0.328 & 0.261 & 0.245 \\
2 & 0.505 & 0.505 & 0.500 & 0.496 & 0.487 \\
3 & 0.719 & 0.720 & 0.719 & 0.713 & 0.708 \\
4 & 1.002 & 1.000 & 0.995 & 0.995 & 0.984 \\
High 5 & 4.053 & 4.064 & 6.684 & 12.808 & 9.688 \\
\hline
\end{tabular}


Table 5. (continued)

Panel E. Average Leverage Ratio

\begin{tabular}{lrrrrr}
\hline & Illiquid 1 & 2 & 3 & 4 & Liquid 5 \\
\hline Low 1 & 0.535 & 0.480 & 0.418 & 0.345 & 0.275 \\
2 & 0.519 & 0.461 & 0.416 & 0.363 & 0.288 \\
3 & 0.472 & 0.459 & 0.427 & 0.377 & 0.299 \\
4 & 0.473 & 0.461 & 0.432 & 0.384 & 0.305 \\
High 5 & 0.470 & 0.461 & 0.447 & 0.410 & 0.328 \\
\hline
\end{tabular}

Panel F. Average Cash Holding Deviation from Industry Norm

\begin{tabular}{lrrrrr}
\hline & Illiquid 1 & 2 & 3 & 4 & Liquid 5 \\
\hline Low 1 & -0.106 & -0.069 & 0.001 & 0.108 & 0.314 \\
2 & -0.106 & -0.084 & -0.039 & 0.042 & 0.238 \\
3 & -0.105 & -0.086 & -0.057 & 0.008 & 0.186 \\
4 & -0.104 & -0.088 & -0.061 & -0.006 & 0.167 \\
High 5 & -0.102 & -0.089 & -0.063 & -0.017 & 0.140 \\
\hline
\end{tabular}

Panel G. Average Leverage Deviation from Industry Norm

\begin{tabular}{lrrrrr}
\hline & Illiquid 1 & 2 & 3 & 4 & Liquid 5 \\
\hline Low 1 & 0.103 & 0.051 & -0.008 & -0.075 & -0.138 \\
2 & 0.047 & 0.029 & -0.013 & -0.063 & -0.134 \\
3 & 0.039 & 0.026 & -0.007 & -0.053 & -0.126 \\
4 & 0.039 & 0.028 & -0.002 & -0.047 & -0.122 \\
High 5 & 0.036 & 0.028 & 0.013 & -0.024 & -0.104 \\
\hline
\end{tabular}

Panel H. Average Cash Flow

\begin{tabular}{lrrrrr}
\hline & Illiquid 1 & 2 & 3 & 4 & Liquid 5 \\
\hline Low 1 & 0.036 & 0.039 & 0.037 & 0.023 & -0.047 \\
2 & 0.038 & 0.074 & 0.075 & 0.072 & 0.030 \\
3 & 0.071 & 0.072 & 0.069 & 0.068 & 0.042 \\
4 & 0.059 & 0.061 & 0.061 & 0.061 & 0.038 \\
High 5 & 0.039 & 0.045 & 0.045 & 0.045 & 0.028 \\
\hline
\end{tabular}

Panel I. Average Cash Flow Volatility

\begin{tabular}{lrrrrrrr}
\hline & Illiquid 1 & 2 & 3 & 4 & Liquid 5 & Liquid - Illiquid & t-statistic \\
\hline Low 1 & 0.677 & 0.650 & 0.697 & 0.786 & 0.881 & 0.204 & 12.363 \\
2 & 0.291 & 0.294 & 0.335 & 0.389 & 0.475 & 0.183 & 16.992 \\
3 & 0.244 & 0.238 & 0.231 & 0.240 & 0.377 & 0.132 & 18.742 \\
4 & 0.171 & 0.191 & 0.204 & 0.207 & 0.329 & 0.158 & 17.300 \\
High 5 & 0.155 & 0.127 & 0.187 & 0.181 & 0.238 & 0.084 & 10.953 \\
\hline
\end{tabular}


Table 5. (continued)

Panel J. Average Beta

\begin{tabular}{lrrrrrrr}
\hline & Illiquid 1 & 2 & 3 & 4 & Liquid 5 & Liquid - Illiquid & t-statistic \\
\hline Low 1 & 0.942 & 1.056 & 1.236 & 1.434 & 1.492 & 0.550 & 12.363 \\
2 & 0.936 & 0.954 & 1.103 & 1.225 & 1.451 & 0.515 & 16.992 \\
3 & 0.892 & 0.919 & 0.968 & 1.119 & 1.354 & 0.462 & 18.742 \\
4 & 0.862 & 0.914 & 0.902 & 1.019 & 1.198 & 0.336 & 17.300 \\
High 5 & 0.846 & 0.839 & 0.878 & 0.934 & 1.048 & 0.202 & 10.953 \\
\hline
\end{tabular}

F-stat for Beta difference of all Liquidity-BE/ME quintiles: $F$-value $=47.84 ; \mathrm{p}$ value $=1.0527 \mathrm{e}-008$

F-stat for Beta difference of most Liquid and illiquid-BE/ME quintiles: $\mathrm{F}$-value $=73.52 ; \mathrm{p}$ value $=0.001$

Panel K. Average Total Assets

\begin{tabular}{lrrrrrrr}
\hline & Illiquid 1 & 2 & 3 & 4 & Liquid 5 & Liquid - Illiquid & t-statistic \\
\hline Low 1 & 1201.411 & 1269.293 & 986.316 & 599.050 & 340.262 & -861.140 & -16.758 \\
2 & 1489.823 & 1546.406 & 1100.109 & 657.953 & 342.413 & -1147.390 & -18.761 \\
3 & 1125.348 & 1390.816 & 1111.067 & 668.044 & 306.124 & -819.180 & -23.496 \\
4 & 983.152 & 1151.628 & 1083.351 & 741.831 & 300.962 & -682.190 & -20.105 \\
High 5 & 854.530 & 1196.312 & 1987.549 & 2249.157 & 1102.396 & 247.470 & 5.599 \\
\hline
\end{tabular}

Panel L. Average KZ index

\begin{tabular}{lrrrrrrr}
\hline & Illiquid 1 & 2 & 3 & 4 & Liquid 5 & Liquid - Illiquid & t-statistic \\
\hline Low 1 & -1.120 & -1.593 & -2.177 & -2.483 & -2.928 & -1.808 & -7.733 \\
2 & -3.226 & -3.332 & -3.392 & -3.299 & -3.783 & -0.558 & -2.653 \\
3 & -4.143 & -4.183 & -4.255 & -4.166 & -4.075 & 0.068 & 0.361 \\
4 & -4.034 & -3.852 & -4.552 & -4.332 & -4.908 & -0.874 & -4.342 \\
High 5 & -3.420 & -4.062 & -3.933 & -4.139 & -4.053 & -0.633 & -2.610 \\
\hline
\end{tabular}

Panel M. Average DivPayout

\begin{tabular}{lrrrrrrr}
\hline & Illiquid 1 & 2 & 3 & 4 & Liquid 5 & Liquid - Illiquid & t-statistic \\
\hline Low 1 & 0.092 & 0.099 & 0.106 & 0.112 & 0.116 & 0.024 & 4.437 \\
2 & 0.127 & 0.128 & 0.126 & 0.116 & 0.116 & -0.011 & -2.019 \\
3 & 0.146 & 0.147 & 0.146 & 0.137 & 0.121 & -0.024 & -4.970 \\
4 & 0.143 & 0.137 & 0.150 & 0.139 & 0.137 & -0.006 & -1.130 \\
High 5 & 0.119 & 0.128 & 0.129 & 0.134 & 0.117 & -0.002 & -0.421 \\
\hline
\end{tabular}

Panel N. Average Distribution to Shareholders

\begin{tabular}{lrrrrrrr}
\hline & Illiquid 1 & 2 & 3 & 4 & Liquid 5 & Liquid - Illiquid & t-statistic \\
\hline Low 1 & 0.158 & 0.179 & 0.164 & 0.158 & 0.144 & -0.014 & -2.105 \\
2 & 0.203 & 0.203 & 0.191 & 0.180 & 0.169 & -0.034 & -6.078 \\
3 & 0.207 & 0.216 & 0.214 & 0.201 & 0.185 & -0.022 & -3.730 \\
4 & 0.191 & 0.190 & 0.229 & 0.204 & 0.210 & 0.019 & 2.760 \\
High 5 & 0.150 & 0.164 & 0.182 & 0.193 & 0.164 & 0.014 & 2.197 \\
\hline
\end{tabular}




\section{Table 6. Regression of Individual Firm Beta on Firm Characteristics}

This table shows the regression results of individual firm's market beta on corporate liquidity (CL), size and book-to-market ratio (BE/ME). The sample period is from 1962:01 to 2005:12. $t$ statistics are in parenthesis.

\begin{tabular}{|c|c|c|c|}
\hline & $\begin{array}{c}\text { Time series average } \\
\text { of cross-sectional } \\
\text { regression }\end{array}$ & Pooled regression & $\begin{array}{c}\text { Cross-sectional } \\
\text { regression on Time } \\
\text { series average }\end{array}$ \\
\hline Constant & $\begin{array}{c}1.191 \\
(68.725)^{* * *}\end{array}$ & $\begin{array}{c}1.079 \\
(1590.100)^{* * *}\end{array}$ & $\begin{array}{c}1.019 \\
(92.185)^{* * *}\end{array}$ \\
\hline$C L$ & $\begin{array}{c}0.296 \\
(8.073)^{* * *}\end{array}$ & $\begin{array}{c}0.993 \\
(369.630)^{* * *}\end{array}$ & $c^{1.506}{ }^{* * *}$ \\
\hline Size & $\begin{array}{c}0.000 \\
(-4.111)^{* * *}\end{array}$ & $\begin{array}{c}0.000 \\
(-22.134)^{* * *}\end{array}$ & $\begin{array}{c}0.000 \\
(0.758)^{* * *}\end{array}$ \\
\hline$B E / M E$ & $\begin{array}{c}-0.051 \\
(-3.920)^{* * *}\end{array}$ & $\begin{array}{r}-0.010 \\
(-59.310)^{* * *}\end{array}$ & $\begin{array}{c}-0.009 \\
(-3.813)^{* * *}\end{array}$ \\
\hline$R^{2}$ & 0.089 & 0.113 & 0.171 \\
\hline$* 90 \%$ sign & ** 95\% significan & $* * * 99 \%$ sign & nce level \\
\hline
\end{tabular}


Table 7. Fama-MacBeth Second-pass Regression on Individual Firms

Panel A. 25 Size-BE/ME portfolios

In the first pass, portfolio excess returns are regressed on market, $S M B$ and $H M L$ factors. Each individual firm is assigned with betas of the portfolio that the firm belongs to. In the second pass, individual stocks' 1 year forward expected excess returns are regressed on factor betas from the first pass, corporate liquidity measures, liquidity constraint dummies or interaction terms of corporate liquidity and liquidity constraint dummies. Liquidity constraint dummies are defined as follows: $\mathrm{D}$ low equals one if the time series average of individual firm's cash holdings falls below the bottom 30 percentile of the industry average level and zero otherwise. D_high equals one if the time series average of individual firm's cash holdings is higher than the top 30 percentile of the industry average level and zero otherwise. CF sigma is the cash flow volatility. Financial constraint measures include KZ index and dividend payout. Total assets is the book value of total assets. KZ index is a linear index of firm financial constraints constructed based on Kaplan and Zingales (1997). Dividend payout is calculated as the ratio of total dividends distributions to operating income. Distribution to Shareholders is defined as the sum of total dividends and stock repurchase divided by operating income.

\begin{tabular}{|c|c|c|c|c|c|c|c|c|}
\hline & Model 1 & Model 2 & Model 3 & Model 4 & Model 5 & Model 6 & Model 7 & Model 8 \\
\hline constant & $\begin{array}{l}-4.482 \\
(-9.768)^{* * *}\end{array}$ & $\begin{array}{l}-4.709 \\
(-10.291)^{* * *}\end{array}$ & $\begin{array}{l}-4.900 \\
(-10.522)^{* * *}\end{array}$ & $\begin{array}{l}-4.660 \\
(-10.281)^{* * *}\end{array}$ & $\begin{array}{l}-5.030 \\
(-11.206)^{* * *}\end{array}$ & $\begin{array}{l}-6.913 \\
(-11.438)^{* * *}\end{array}$ & $\begin{array}{l}-6.952 \\
(-11.486)^{* * *}\end{array}$ & $\begin{array}{l}-6.955 \\
(-11.495)^{* * *}\end{array}$ \\
\hline$b$ (Market beta) & $\begin{array}{l}5.208 \\
(10.852)^{* * *}\end{array}$ & $\begin{array}{l}5.293 \\
(11.176)^{* * *}\end{array}$ & $\begin{array}{l}5.365 \\
(11.276)^{* * *}\end{array}$ & $\begin{array}{l}5.252 \\
(11.144)^{* * *}\end{array}$ & $\begin{array}{l}5.528 \\
(11.821)^{* * *}\end{array}$ & $\begin{array}{l}8.885 \\
(13.702)^{* * *}\end{array}$ & $\begin{array}{l}8.931 \\
(13.743)^{* * *}\end{array}$ & $\begin{array}{l}8.932 \\
(13.759)^{* * *}\end{array}$ \\
\hline$s$ (Size beta) & $\begin{array}{l}-0.057 \\
(-0.290)\end{array}$ & $\begin{array}{l}-0.008 \\
(-0.042)\end{array}$ & $\begin{array}{l}-0.020 \\
(-0.107)\end{array}$ & $\begin{array}{l}-0.003 \\
(-0.018)\end{array}$ & $\begin{array}{l}-0.013 \\
(-0.073)\end{array}$ & $\begin{array}{l}0.276 \\
(0.157)\end{array}$ & $\begin{array}{l}-0.171 \\
(0.126)\end{array}$ & $\begin{array}{l}0.175 \\
(0.140)\end{array}$ \\
\hline$h$ (Value beta) & $\begin{array}{l}2.513 \\
(14.409)^{* * *}\end{array}$ & $\begin{array}{l}2.401 \\
(16.469)^{* * *}\end{array}$ & $\begin{array}{l}2.389 \\
(16.385)^{* * *}\end{array}$ & $\begin{array}{l}2.390 \\
(16.467)^{\text {*** }}\end{array}$ & $\begin{array}{l}2.402 \\
(17.678)^{* * *}\end{array}$ & $\begin{array}{l}4.310 \\
(17.038)^{* * *}\end{array}$ & $\begin{array}{l}4.305 \\
(16.849)^{* * *}\end{array}$ & $\begin{array}{l}4.209 \\
(16.826)^{* * *}\end{array}$ \\
\hline$C L$ & - & $\begin{array}{l}1.465 \\
(4.811)^{* * *}\end{array}$ & $\begin{array}{l}3.459 \\
(5.518)^{* * *}\end{array}$ & $\begin{array}{l}1.346 \\
(4.472)^{* * *}\end{array}$ & $\begin{array}{l}1.399 \\
(5.176)^{* * *}\end{array}$ & $\begin{array}{l}1.073 \\
(3.029)^{* * *}\end{array}$ & $\begin{array}{l}1.123 \\
(3.118)^{* * *}\end{array}$ & $\begin{array}{l}1.005 \\
(2.789)^{* * *}\end{array}$ \\
\hline CL squared & - & - & $\begin{array}{l}-4.948 \\
(-3.683)^{* * *}\end{array}$ & - & - & - & - & - \\
\hline$C L \times C F \_$sigma & - & - & - & $\begin{array}{l}0.311 \\
(1.822)^{*}\end{array}$ & $\begin{array}{l}0.310 \\
(1.821)^{*}\end{array}$ & - & - & - \\
\hline With industry & - & - & - & - & & - & - & - \\
\hline dummies & - & - & - & - & & - & - & - \\
\hline$C L \times K Z$ Index & - & - & - & - & - & $\begin{array}{l}0.086 \\
(5.184)^{* * *}\end{array}$ & - & - \\
\hline$C L \times$ Dividend & - & - & - & - & - & - & -2.922 & - \\
\hline Payout & - & - & - & - & - & - & $(-4.508)^{* * *}$ & - \\
\hline$C L \times$ Distribution & - & - & - & - & - & - & - & -1.883 \\
\hline to Shareholders & - & - & - & - & - & - & - & $(-3.895)^{* * *}$ \\
\hline$R^{2}$ & 0.047 & 0.052 & 0.053 & 0.054 & 0.088 & 0.050 & 0.048 & 0.049 \\
\hline
\end{tabular}

$* 90 \%$ significance level $\quad * * 95 \%$ significance level $\quad * * * 99 \%$ significance level 
Table 7. (Continued)

Panel B. 27 Size-BE/ME-Corporate Liquidity portfolios

In the first pass, portfolio excess returns are regressed on market, $S M B$ and $H M L$ factors. Each individual firm is assigned with betas of the portfolio that the firm belongs to. In the second pass, individual stocks' 1 year forward expected excess returns are regressed on factor betas from the first pass, corporate liquidity measures, liquidity constraint dummies or interaction terms of corporate liquidity and liquidity constraint dummies. Liquidity constraint dummies are defined as follows: $\mathrm{D}$ low equals one if the time series average of individual firm's cash holdings falls below the bottom 30 percentile of the industry average level and zero otherwise. D_high equals one if the time series average of individual firm's cash holdings is higher than the top 30 percentile of the industry average level and zero otherwise. Financial constraint measures include KZ index and dividend payout. Total assets is the book value of total assets. KZ index is a linear index of firm financial constraints constructed based on Kaplan and Zingales (1997). Dividend payout is calculated as the ratio of total dividends distributions to operating income. Distribution to Shareholders is defined as the sum of total dividends and stock repurchase divided by operating income.

\begin{tabular}{|c|c|c|c|c|c|c|c|c|}
\hline & Model 1 & Model 2 & Model 3 & Model 4 & Model 5 & Model 6 & Model 7 & Model 8 \\
\hline constant & $\begin{array}{l}-4.934 \\
(-7.586)^{* * *}\end{array}$ & $\begin{array}{l}-5.016 \\
(-9.995)^{* * *}\end{array}$ & $\begin{array}{l}-4.892 \\
(-9.564)^{* * *}\end{array}$ & $\begin{array}{l}-4.821 \\
(-9.598)^{* * *}\end{array}$ & $\begin{array}{l}-5.335 \\
(-10.985)^{* * *}\end{array}$ & $\begin{array}{l}-5.674 \\
(-10.893)^{* * *}\end{array}$ & $\begin{array}{l}-4.891 \\
(-9.654)^{* * *}\end{array}$ & $\begin{array}{l}-4.506 \\
(-8.512)^{\text {*** }}\end{array}$ \\
\hline$b$ (Market beta) & $\begin{array}{l}5.847 \\
(9.934)^{* * *}\end{array}$ & $\begin{array}{l}5.885 \\
(11.404)^{* * *}\end{array}$ & $\begin{array}{l}5.811 \\
(11.177)^{* * *}\end{array}$ & $\begin{array}{l}5.383 \\
(10.304)^{* * *}\end{array}$ & $\begin{array}{l}6.134 \\
(12.187)^{* * *}\end{array}$ & $\begin{array}{l}6.495 \\
(12.401)^{* * *}\end{array}$ & $\begin{array}{l}6.557 \\
(12.981)^{* * *}\end{array}$ & $\begin{array}{l}5.753 \\
(11.859)^{* * *}\end{array}$ \\
\hline$s$ (Size beta) & $\begin{array}{l}-0.089 \\
(-0.350)\end{array}$ & $\begin{array}{l}-0.101 \\
(-0.541)\end{array}$ & $\begin{array}{l}-0.087 \\
(-0.470)\end{array}$ & $\begin{array}{l}-0.071 \\
(-0.448)\end{array}$ & $\begin{array}{l}-0.119 \\
(-0.668)\end{array}$ & $\begin{array}{l}0.198 \\
(0.657)\end{array}$ & $\begin{array}{l}-0.103 \\
(-0.826)\end{array}$ & $\begin{array}{l}0.114 \\
(0.776)\end{array}$ \\
\hline$h$ (Value beta) & $\begin{array}{l}2.568 \\
(12.495)^{* * *}\end{array}$ & $\begin{array}{l}2.393 \\
(17.323)^{* * *}\end{array}$ & $\begin{array}{l}2.426 \\
(17.108)^{\text {*** }}\end{array}$ & $\begin{array}{l}2.111 \\
(16.745)^{* * *}\end{array}$ & $\begin{array}{l}2.372 \\
(18.775)^{* * *}\end{array}$ & $\begin{array}{l}2.492 \\
(17.002)^{* * *}\end{array}$ & $\begin{array}{l}2.562 \\
(18.168)^{* * *}\end{array}$ & $\begin{array}{l}3.015 \\
(18.112)^{* * *}\end{array}$ \\
\hline$C L$ & - & $\begin{array}{l}1.640 \\
(3.998)^{* * *}\end{array}$ & $\begin{array}{l}3.452 \\
(2.110)^{* *}\end{array}$ & $\begin{array}{l}1.245 \\
(2.209)^{* *}\end{array}$ & $\begin{array}{l}1.922 \\
(3.364)^{* * *}\end{array}$ & $\begin{array}{l}2.573 \\
(3.938)^{* * *}\end{array}$ & $\begin{array}{l}2.667 \\
(3.765)^{* * *}\end{array}$ & $\begin{array}{l}2.349 \\
(4.028)^{* * *}\end{array}$ \\
\hline CL squared & $\begin{array}{l}- \\
-\end{array}$ & - & $\begin{array}{l}-3.539 \\
(-2.148)\end{array}$ & - & - & - & - & - \\
\hline$C L \times C F_{\text {sigma }}$ & - & - & - & $\begin{array}{l}0.305 \\
(2.148)^{* *}\end{array}$ & $\begin{array}{l}0.879 \\
(4.598)^{* * *}\end{array}$ & - & - & - \\
\hline With industry & - & - & - & - & & - & - & - \\
\hline dummies & - & - & - & - & & - & - & - \\
\hline$C L \times K Z$ Index & - & - & - & - & - & $\begin{array}{l}0.094 \\
(6.003)^{* * *}\end{array}$ & - & - \\
\hline$C L \times$ Dividend & - & - & - & - & - & - & -2.712 & - \\
\hline Payout & - & - & - & - & - & - & $(-3.902)^{* * *}$ & - \\
\hline$C L \times$ Distribution & - & - & - & - & - & - & - & -2.426 \\
\hline to Shareholders & - & - & - & - & - & - & - & $(-2.590)^{* *}$ \\
\hline$R^{2}$ & 0.044 & 0.045 & 0.048 & 0.065 & 0.084 & 0.052 & 0.050 & 0.056 \\
\hline
\end{tabular}

$* 90 \%$ significance level

$* * 95 \%$ significance level

$* * * 99 \%$ significance level 


\section{Table 8. Distribution of Firms across Industries and Corporate Liquidity / Corporate Liquidity Volatility Portfolios}

Each firm is assigned to one of the Fama-French 17 industries based on its CRSP four-digit SIC code. Deviation of the firm's liquidity and leverage to industry norm are estimated as the difference between liquidity and leverage of that firm and the mean level of the industry that the firm belongs to. Corporate liquidity volatility is measured as the time-series standard deviation of each firm. Stocks are sorted into quintiles on the basis of corporate liquidity and corporate liquidity volatility. Panel A shows the distribution of firms on the basis of corporate liquidity, which is measured as the ratio of cash and cash equivalents over total assets. Panel B displays the distribution of firms on the basis of corporate liquidity volatility. Industry average of corporate liquidity and corporate liquidity volatility are displayed in the last column for each panel respectively.

Panel A. Numbers of Firms in 17 Industries and Corporate Liquidity Portfolios

\begin{tabular}{|c|c|c|c|c|c|c|c|}
\hline & & low CL 1 & 2 & 3 & 4 & high CL 5 & $\begin{array}{c}\text { Average Cash-to- } \\
\text { asset ratio }\end{array}$ \\
\hline 1 & & 26 & 20 & 19 & 18 & 12 & 0.105 \\
\hline 2 & & 9 & 9 & 10 & 9 & 10 & 0.132 \\
\hline 3 & & 32 & 30 & 28 & 20 & 11 & 0.107 \\
\hline 4 & & 26 & 19 & 15 & 12 & 9 & 0.096 \\
\hline 5 & & 24 & 21 & 21 & 17 & 14 & 0.123 \\
\hline 6 & & 15 & 15 & 12 & 9 & 7 & 0.124 \\
\hline 7 & & 10 & 14 & 18 & 21 & 40 & 0.366 \\
\hline 8 & & 27 & 31 & 29 & 20 & 13 & 0.087 \\
\hline 9 & & 18 & 15 & 13 & 9 & 4 & 0.090 \\
\hline 10 & & 10 & 9 & 6 & 5 & 3 & 0.076 \\
\hline 11 & & 63 & 71 & 75 & 93 & 88 & 0.186 \\
\hline 12 & & 13 & 12 & 11 & 9 & 4 & 0.099 \\
\hline 13 & & 10 & 10 & 8 & 6 & 5 & 0.098 \\
\hline & $*$ & $\mathrm{~N} / \mathrm{A}$ & N/A & N/A & N/A & N/A & N/A \\
\hline 15 & & 34 & 39 & 38 & 34 & 20 & 0.102 \\
\hline 16 & $*$ & N/A & N/A & N/A & N/A & N/A & N/A \\
\hline 17 & & 104 & 108 & 120 & 140 & 182 & 0.210 \\
\hline
\end{tabular}


Table 8. (Continued)

Panel B. Numbers of Firms in 17 Industries and Corporate Liquidity Volatility Portfolios Average Corporate

\begin{tabular}{|c|c|c|c|c|c|c|c|}
\hline & & lity 1 & 2 & 3 & 4 & high Volatility 5 & Liquidity Volatility \\
\hline 1 & & 27 & 24 & 22 & 13 & 8 & 0.095 \\
\hline 2 & & 6 & 10 & 13 & 11 & 8 & 0.128 \\
\hline 3 & & 32 & 39 & 26 & 15 & 10 & 0.082 \\
\hline 4 & & 26 & 20 & 15 & 12 & 8 & 0.085 \\
\hline 5 & & 25 & 26 & 16 & 17 & 12 & 0.102 \\
\hline 6 & & 12 & 24 & 11 & 5 & 4 & 0.093 \\
\hline 7 & & 8 & 18 & 24 & 27 & 25 & 0.210 \\
\hline 8 & & 29 & 41 & 30 & 13 & 6 & 0.088 \\
\hline 9 & & 16 & 20 & 15 & 6 & 2 & 0.069 \\
\hline 10 & & 9 & 11 & 8 & 3 & 2 & 0.080 \\
\hline 11 & & 52 & 80 & 86 & 87 & 85 & 0.141 \\
\hline 12 & & 13 & 16 & 12 & 7 & 2 & 0.081 \\
\hline 13 & & 9 & 11 & 8 & 7 & 4 & 0.091 \\
\hline 14 & $*$ & N/A & N/A & N/A & N/A & N/A & N/A \\
\hline 15 & & 35 & 47 & 44 & 27 & 12 & 0.102 \\
\hline 16 & * & N/A & N/A & N/A & N/A & N/A & N/A \\
\hline 17 & & 84 & 118 & 130 & 154 & 167 & 0.159 \\
\hline
\end{tabular}

* Industry 14 is utilities and 16 is banks, insurance companies and other financials, which are excluded from the sample because the liquid assets held by utility industry are under certain regulation while financial industry usually hold a substantial inventory of marketable securities.

Notes:

1 Food Food

2 Mines Mining and Minerals

3 Oil Oil and Petroleum Products

4 Clths Textiles, Apparel \& Footware

5 Durbl Consumer Durables

6 Chems Chemicals

7 Cnsum Drugs, Soap, Prfums,

Tobacco

8 Cnstr Construction and Construction

Materials

9 Steel Steel Works Etc

10 FabPr Fabricated Products

11 Machn Machinery and Business Equipment

12 Cars Automobiles

13 Trans Transportation

14 Utils Utilities

15 Rtail Retail Stores

16 Finan Banks, Insurance Companies, and Other Financials

17 Other Everything Else 


\section{Table A1. Size Effect Controlled by Corporate Liquidity}

From 1985.7 to 2003.12 , at the beginning of each month, stocks are sorted into 5 portfolios on the basis of corporate liquidity. Within each liquidity portfolios, stocks are subsequently sorted into 5 size quintiles. The equally weighted averages of return, corporate liquidity, size, BE/ME, leverage, deviations of corporate liquidity and leverage from industry norm, beta and alternative financial constraint measures are reported. "Small-Big" is the differences in the returns between the smallest and biggest size portfolios.

Panel A. Average Return*

\begin{tabular}{lrrrrrrr}
\hline & Small1 & 2 & 3 & 4 & Big 5 & Small - Big & t-statistics \\
\hline Illiquid 1 & 1.748 & 0.684 & 0.671 & 0.817 & 0.779 & 0.969 & 2.252 \\
2 & 1.972 & 0.922 & 0.866 & 0.830 & 0.842 & 1.130 & 2.568 \\
3 & 2.340 & 1.024 & 1.017 & 1.100 & 0.961 & 1.380 & 3.072 \\
4 & 2.760 & 1.499 & 1.312 & 1.293 & 1.073 & 1.688 & 3.553 \\
Liquid 5 & 3.097 & 1.867 & 1.607 & 1.387 & 1.277 & 1.820 & 3.435 \\
\hline
\end{tabular}

* Average return is monthly stock returns measured in percentage terms

Panel B. Average Corporate Liquidity

\begin{tabular}{lrrrrr}
\hline & Small1 & 2 & 3 & 4 & Big 5 \\
\hline Illiquid 1 & 0.014 & 0.015 & 0.016 & 0.016 & 0.015 \\
2 & 0.039 & 0.039 & 0.039 & 0.038 & 0.038 \\
3 & 0.080 & 0.080 & 0.080 & 0.080 & 0.079 \\
4 & 0.161 & 0.163 & 0.163 & 0.162 & 0.160 \\
Liquid 5 & 0.386 & 0.387 & 0.385 & 0.382 & 0.345 \\
\hline
\end{tabular}

Panel C. Average Size**

\begin{tabular}{lrrrrr}
\hline & Small1 & 2 & 3 & 4 & Big 5 \\
\hline Illiquid 1 & 11.291 & 38.353 & 107.680 & 336.950 & 3276.000 \\
2 & 12.045 & 42.908 & 124.800 & 374.210 & 4875.500 \\
3 & 12.554 & 42.371 & 115.500 & 348.930 & 4740.600 \\
4 & 13.436 & 43.274 & 106.880 & 292.880 & 3942.000 \\
Liquid 5 & 13.902 & 41.105 & 91.621 & 228.290 & 2757.400 \\
\hline **Firms size is
\end{tabular}

**Firms size is in millions of dollars

Panel D. Average BE/ME

\begin{tabular}{lrrrrr}
\hline & Small1 & 2 & 3 & 4 & Big 5 \\
\hline Illiquid 1 & 3.160 & 1.083 & 0.941 & 0.816 & 0.655 \\
2 & 2.890 & 1.432 & 1.263 & 0.904 & 0.639 \\
3 & 6.885 & 2.047 & 1.333 & 1.117 & 0.703 \\
4 & 7.546 & 2.281 & 1.571 & 1.172 & 0.693 \\
Liquid 5 & 2.117 & 0.793 & 1.056 & 0.788 & 0.436 \\
\hline
\end{tabular}


Table A1. (continued)

Panel E. Average Leverage Ratio

\begin{tabular}{lrrrrr}
\hline & Small1 & 2 & 3 & 4 & Big 5 \\
\hline Illiquid 1 & 0.706 & 0.532 & 0.487 & 0.464 & 0.446 \\
2 & 0.551 & 0.499 & 0.481 & 0.451 & 0.436 \\
3 & 0.512 & 0.468 & 0.442 & 0.417 & 0.398 \\
4 & 0.463 & 0.406 & 0.389 & 0.370 & 0.356 \\
Liquid 5 & 0.483 & 0.308 & 0.303 & 0.288 & 0.304 \\
\hline
\end{tabular}

Panel F. Average Cash Holding Deviation from Industry Norm

\begin{tabular}{lrrrrr}
\hline & Small1 & 2 & 3 & 4 & Big 5 \\
\hline Illiquid 1 & -0.112 & -0.107 & -0.103 & -0.102 & -0.102 \\
2 & -0.090 & -0.087 & -0.084 & -0.085 & -0.082 \\
3 & -0.054 & -0.053 & -0.050 & -0.048 & -0.050 \\
4 & 0.020 & 0.020 & 0.023 & 0.022 & 0.018 \\
Liquid 5 & 0.234 & 0.229 & 0.227 & 0.223 & 0.187 \\
\hline
\end{tabular}

Panel G. Average Leverage Deviation from Industry Norm

\begin{tabular}{lrrrrr}
\hline & Small1 & 2 & 3 & 4 & Big 5 \\
\hline Illiquid 1 & 0.230 & 0.091 & 0.053 & 0.031 & 0.013 \\
2 & 0.116 & 0.066 & 0.048 & 0.017 & 0.003 \\
3 & 0.075 & 0.036 & 0.008 & -0.015 & -0.028 \\
4 & 0.027 & -0.025 & -0.043 & -0.058 & -0.065 \\
Liquid 5 & -0.102 & -0.119 & -0.120 & -0.133 & -0.109 \\
\hline
\end{tabular}

Panel H. Average Cash Flow

\begin{tabular}{lrrrrr}
\hline & Small1 & 2 & 3 & 4 & Big 5 \\
\hline Illiquid 1 & -0.124 & 0.035 & 0.061 & 0.079 & 0.086 \\
2 & -0.021 & 0.037 & 0.048 & 0.080 & 0.088 \\
3 & -0.031 & 0.027 & 0.060 & 0.080 & 0.093 \\
4 & -0.076 & 0.021 & 0.053 & 0.080 & 0.102 \\
Liquid 5 & -0.234 & -0.040 & 0.003 & 0.042 & 0.098 \\
\hline
\end{tabular}

\begin{tabular}{lrrrrr}
\hline \multicolumn{6}{l}{ Panel I. Average Cash Flow Volatility } \\
\hline \multicolumn{1}{l}{ Small1 } & 2 & 3 & 4 & Big 5 \\
\hline Illiquid 1 & 0.347 & 0.324 & 0.230 & 0.195 & 0.217 \\
2 & 0.300 & 0.305 & 0.230 & 0.240 & 0.254 \\
3 & 0.334 & 0.321 & 0.289 & 0.258 & 0.221 \\
4 & 0.372 & 0.348 & 0.359 & 0.351 & 0.345 \\
Liquid 5 & 0.874 & 0.608 & 0.528 & 0.569 & 0.552 \\
\hline
\end{tabular}


Table A1. (continued)

Panel J. Average Beta

\begin{tabular}{lrrrrr}
\hline & Small1 & 2 & 3 & 4 & Big 5 \\
\hline Illiquid 1 & 0.885 & 0.856 & 0.840 & 0.820 & 0.839 \\
2 & 0.924 & 0.903 & 0.879 & 0.885 & 0.858 \\
3 & 1.054 & 1.037 & 1.034 & 1.190 & 0.957 \\
4 & 1.302 & 1.320 & 1.295 & 1.285 & 1.186 \\
Liquid 5 & 1.367 & 1.416 & 1.430 & 1.416 & 1.345 \\
\hline
\end{tabular}

Panel K. Average Total Assets

\begin{tabular}{lrrrrr}
\hline & Small1 & 2 & 3 & 4 & Big 5 \\
\hline Illiquid 1 & 61.65 & 125.00 & 290.45 & 728.14 & 4185.20 \\
2 & 113.31 & 222.05 & 544.08 & 1055.30 & 5308.92 \\
3 & 234.28 & 334.44 & 462.62 & 1137.30 & 4602.34 \\
4 & 164.96 & 211.30 & 392.93 & 705.39 & 2986.04 \\
Liquid 5 & 41.41 & 64.04 & 183.98 & 309.37 & 1415.41 \\
\hline
\end{tabular}

Panel L. Average KZ index

\begin{tabular}{lrrrrr}
\hline & Small1 & 2 & 3 & 4 & Big 5 \\
\hline Illiquid 1 & 0.175 & -2.118 & -3.282 & -4.817 & -6.873 \\
2 & -0.811 & -2.265 & -3.688 & -4.497 & -6.606 \\
3 & -0.520 & -2.181 & -3.792 & -5.037 & -7.000 \\
4 & -1.087 & -2.509 & -3.623 & -4.645 & -6.043 \\
Liquid 5 & -1.954 & -2.633 & -3.998 & -4.716 & -5.052 \\
\hline
\end{tabular}

Panel M. Average DivPayout

\begin{tabular}{lrrrrr}
\hline & Small1 & 2 & 3 & 4 & Big 5 \\
\hline Illiquid 1 & 0.047 & 0.093 & 0.122 & 0.159 & 0.211 \\
2 & 0.054 & 0.096 & 0.132 & 0.156 & 0.213 \\
3 & 0.057 & 0.095 & 0.133 & 0.171 & 0.215 \\
4 & 0.056 & 0.095 & 0.124 & 0.150 & 0.193 \\
Liquid 5 & 0.072 & 0.086 & 0.118 & 0.144 & 0.160 \\
\hline
\end{tabular}

Panel N. Average Distribution to Shareholders

\begin{tabular}{lrrrrr}
\hline & Small1 & 2 & 3 & 4 & Big 5 \\
\hline Illiquid 1 & 0.071 & 0.137 & 0.166 & 0.216 & 0.305 \\
2 & 0.093 & 0.165 & 0.187 & 0.217 & 0.311 \\
3 & 0.097 & 0.142 & 0.196 & 0.247 & 0.320 \\
4 & 0.086 & 0.151 & 0.194 & 0.215 & 0.294 \\
Liquid 5 & 0.097 & 0.122 & 0.161 & 0.200 & 0.241 \\
\hline
\end{tabular}




\section{Table A2. Value Effect Controlled by Corporate Liquidity}

From 1985.7 to 2003.12, at the beginning of each month, stocks are sorted into 5 portfolios on the basis of corporate liquidity. Within each liquidity portfolios, stocks are subsequently sorted into $5 \mathrm{BE} / \mathrm{ME}$ quintiles. The equally weighted averages of return, corporate liquidity, size, $\mathrm{BE} / \mathrm{ME}$, leverage, deviations of corporate liquidity and leverage from industry norm, beta and alternative financial constraint measures are reported. "High-Low" is the differences in the returns between the highest $\mathrm{BE} / \mathrm{ME}$ and lowest $\mathrm{BE} / \mathrm{ME}$ portfolios.

Panel A. Average Return*

\begin{tabular}{lrrrrrrr}
\hline & Low1 & 2 & 3 & 4 & High 5 & High - Low & t-statistics \\
\hline Illiquid 1 & -0.033 & 0.367 & 0.880 & 1.249 & 2.440 & 2.473 & 5.894 \\
2 & 0.012 & 0.553 & 1.025 & 1.543 & 2.515 & 2.503 & 5.892 \\
3 & 0.195 & 0.657 & 1.247 & 1.736 & 2.822 & 2.627 & 5.974 \\
4 & 0.332 & 0.959 & 1.495 & 2.031 & 3.185 & 2.853 & 6.216 \\
Liquid 5 & 0.452 & 1.006 & 1.742 & 2.432 & 3.605 & 3.153 & 6.225 \\
\hline
\end{tabular}

* Average return is monthly stock returns measured in percentage terms

Panel B. Average Corporate Liquidity

\begin{tabular}{lrrrrr}
\hline & Low1 & 2 & 3 & 4 & High 5 \\
\hline Illiquid 1 & 0.015 & 0.015 & 0.015 & 0.015 & 0.015 \\
2 & 0.040 & 0.038 & 0.039 & 0.038 & 0.039 \\
3 & 0.083 & 0.081 & 0.080 & 0.080 & 0.080 \\
4 & 0.167 & 0.165 & 0.164 & 0.162 & 0.160 \\
Liquid 5 & 0.402 & 0.392 & 0.381 & 0.366 & 0.364 \\
\hline
\end{tabular}

Panel C. Average Size**

\begin{tabular}{lrrrrr}
\hline & Low1 & 2 & 3 & 4 & High 5 \\
\hline Illiquid 1 & 1757.300 & 935.130 & 609.190 & 417.890 & 213.510 \\
2 & 2749.900 & 1379.000 & 871.680 & 508.250 & 226.010 \\
3 & 2606.200 & 1322.100 & 793.190 & 519.170 & 264.250 \\
4 & 2205.300 & 1143.100 & 582.340 & 363.040 & 198.850 \\
Liquid 5 & 1445.900 & 832.980 & 504.740 & 347.500 & 112.490 \\
\hline
\end{tabular}

**Firms size is in millions of dollars

Panel D. Average BE/ME

\begin{tabular}{lrrrrr}
\hline & Low1 & 2 & 3 & 4 & High 5 \\
\hline Illiquid 1 & 0.276 & 0.565 & 0.766 & 1.054 & 4.470 \\
2 & 0.281 & 0.567 & 0.757 & 1.052 & 5.150 \\
3 & 0.248 & 0.527 & 0.726 & 1.005 & 10.390 \\
4 & 0.288 & 0.464 & 0.660 & 0.909 & 11.508 \\
Liquid 5 & 0.172 & 0.357 & 0.517 & 0.737 & 3.577 \\
\hline
\end{tabular}


Table A2. (continued)

Panel E. Average Leverage Ratio

\begin{tabular}{lrrrrr}
\hline & Low1 & 2 & 3 & 4 & High 5 \\
\hline Illiquid 1 & 0.528 & 0.520 & 0.477 & 0.474 & 0.469 \\
2 & 0.499 & 0.463 & 0.462 & 0.458 & 0.460 \\
3 & 0.463 & 0.431 & 0.420 & 0.418 & 0.431 \\
4 & 0.408 & 0.378 & 0.369 & 0.364 & 0.387 \\
Liquid 5 & 0.314 & 0.294 & 0.292 & 0.286 & 0.292 \\
\hline
\end{tabular}

Panel F. Average Cash Holding Deviation from Industry Norm

\begin{tabular}{lrrrrr}
\hline & Low1 & 2 & 3 & 4 & High 5 \\
\hline Illiquid 1 & -0.110 & -0.107 & -0.105 & -0.102 & -0.100 \\
2 & -0.091 & -0.089 & -0.085 & -0.084 & -0.081 \\
3 & -0.058 & -0.054 & -0.049 & -0.048 & -0.044 \\
4 & 0.018 & 0.020 & 0.023 & 0.025 & 0.027 \\
Liquid 5 & 0.239 & 0.228 & 0.220 & 0.212 & 0.215 \\
\hline
\end{tabular}

Panel G. Average Leverage Deviation from Industry Norm

\begin{tabular}{lrrrrr}
\hline & Low1 & 2 & 3 & 4 & High 5 \\
\hline Illiquid 1 & 0.098 & 0.042 & 0.043 & 0.040 & 0.034 \\
2 & 0.068 & 0.027 & 0.027 & 0.025 & 0.026 \\
3 & 0.034 & -0.003 & -0.017 & -0.015 & -0.004 \\
4 & -0.018 & -0.051 & -0.061 & -0.065 & -0.048 \\
Liquid 5 & -0.104 & -0.129 & -0.132 & -0.137 & -0.137 \\
\hline
\end{tabular}

Panel H. Average Cash Flow

\begin{tabular}{lrrrrr}
\hline & Low 1 & 2 & 3 & 4 & High 5 \\
\hline Illiquid 1 & 0.046 & 0.034 & 0.069 & 0.058 & 0.039 \\
2 & 0.048 & 0.075 & 0.069 & 0.058 & 0.045 \\
3 & 0.041 & 0.073 & 0.068 & 0.061 & 0.043 \\
4 & 0.025 & 0.070 & 0.068 & 0.062 & 0.045 \\
Liquid 5 & -0.066 & 0.019 & 0.037 & 0.039 & 0.021 \\
\hline
\end{tabular}

\begin{tabular}{lcrrrr}
\hline \multicolumn{2}{l}{ Panel I. Average Cash Flow Volatility } & & & \\
\hline \multicolumn{7}{r}{ Low 1 } & 2 & 3 & 4 & High 5 \\
\hline 2 & 0.320 & 0.327 & 0.272 & 0.245 & 0.153 \\
3 & 0.343 & 0.291 & 0.294 & 0.231 & 0.145 \\
4 & 0.374 & 0.296 & 0.366 & 0.259 & 0.177 \\
Liquid 5 & 0.492 & 0.416 & 0.358 & 0.279 & 0.205 \\
\hline
\end{tabular}


Table A2. (continued)

Panel J. Average Beta

\begin{tabular}{lrrrrr}
\hline & Low1 & 2 & 3 & 4 & High 5 \\
\hline Illiquid 1 & 0.905 & 0.892 & 0.876 & 0.886 & 0.843 \\
2 & 0.978 & 0.935 & 0.919 & 0.873 & 0.862 \\
3 & 1.086 & 1.007 & 0.982 & 0.915 & 0.897 \\
4 & 1.295 & 1.220 & 1.173 & 1.057 & 0.995 \\
Liquid 5 & 1.491 & 1.438 & 1.341 & 1.246 & 1.145 \\
\hline
\end{tabular}

Panel K. Average Total Assets

\begin{tabular}{lrrrrr}
\hline & Low1 & 2 & 3 & 4 & High 5 \\
\hline Illiquid 1 & 1196.91 & 1191.70 & 1092.28 & 973.22 & 890.93 \\
2 & 1555.63 & 1525.67 & 1306.14 & 1127.83 & 1690.41 \\
3 & 1264.52 & 1190.19 & 1058.72 & 993.55 & 2261.82 \\
4 & 839.24 & 824.62 & 613.49 & 548.66 & 1609.56 \\
Liquid 5 & 433.54 & 443.32 & 329.97 & 264.21 & 571.08 \\
\hline
\end{tabular}

Panel L. Average KZ index

\begin{tabular}{lrrrrr}
\hline & Low1 & 2 & 3 & 4 & High 5 \\
\hline Illiquid 1 & -1.503 & -3.650 & -3.995 & -4.002 & -3.716 \\
2 & -2.003 & -3.575 & -4.291 & -3.839 & -4.120 \\
3 & -2.015 & -3.777 & -4.290 & -4.499 & -3.904 \\
4 & -2.217 & -3.272 & -4.098 & -4.276 & -4.038 \\
Liquid 5 & -2.396 & -3.169 & -3.921 & -4.437 & -4.438 \\
\hline
\end{tabular}

Panel M. Average DivPayout

\begin{tabular}{lrrrrr}
\hline & Low1 & 2 & 3 & 4 & High 5 \\
\hline Illiquid 1 & 0.097 & 0.135 & 0.141 & 0.141 & 0.124 \\
2 & 0.108 & 0.133 & 0.149 & 0.136 & 0.128 \\
3 & 0.104 & 0.136 & 0.145 & 0.146 & 0.131 \\
4 & 0.109 & 0.118 & 0.136 & 0.134 & 0.125 \\
Liquid 5 & 0.115 & 0.109 & 0.119 & 0.129 & 0.120 \\
\hline
\end{tabular}

Panel N. Average Distribution to Shareholders

\begin{tabular}{lrrrrr}
\hline & Low1 & 2 & 3 & 4 & High 5 \\
\hline Illiquid 1 & 0.164 & 0.202 & 0.196 & 0.186 & 0.154 \\
2 & 0.189 & 0.207 & 0.219 & 0.189 & 0.172 \\
3 & 0.175 & 0.204 & 0.209 & 0.221 & 0.185 \\
4 & 0.166 & 0.182 & 0.208 & 0.210 & 0.177 \\
Liquid 5 & 0.143 & 0.153 & 0.173 & 0.191 & 0.174 \\
\hline
\end{tabular}


Table A3. Fama-MacBeth Second-pass Regression on Individual Firms

\section{Panel A. 25 Size-BE/ME portfolios}

In the first pass, portfolio excess returns are regressed on market, $S M B$ and $H M L$ factors. Each individual firm is assigned with betas of the portfolio that the firm belongs to. In the second pass, individual stocks' 1 year forward expected excess returns are regressed on factor betas from the first pass, corporate liquidity measures, liquidity constraint dummies or interaction terms of corporate liquidity and liquidity constraint dummies. Liquidity constraint dummies are defined as follows: D_low equals one if the time series average of individual firm's cash holdings falls below the bottom 30 percentile of the industry average level and zero otherwise. D_high equals one if the time series average of individual firm's cash holdings is higher than the top 30 percentile of the industry average level and zero otherwise. CF_sigma is the cash flow volatility.

\begin{tabular}{|c|c|c|c|c|c|c|}
\hline & Model 8 & Model 9 & Model 10 & Model 11 & Model 12 & Model 13 \\
\hline constant & $\begin{array}{l}-4.726 \\
(-10.350)^{* * *}\end{array}$ & $\begin{array}{l}-4.718 \\
(-10.321)^{* * *}\end{array}$ & $\begin{array}{l}-4.699 \\
(-10.253)^{* * *}\end{array}$ & $\begin{array}{l}-4.799 \\
(-10.540)^{* * *}\end{array}$ & $\begin{array}{l}-4.710 \\
(-10.293)^{* * *}\end{array}$ & $\begin{array}{l}-4.804 \\
(-10.561)^{* * *}\end{array}$ \\
\hline$b$ (Market beta) & $\begin{array}{l}5.285 \\
(11.156)^{* * *}\end{array}$ & $\begin{array}{l}5.279 \\
(11.153)^{* * *}\end{array}$ & $\begin{array}{l}5.294 \\
(11.177)^{* * *}\end{array}$ & $\begin{array}{l}5.333 \\
(11.291)^{* * *}\end{array}$ & $\begin{array}{l}5.289 \\
(11.163)^{* * *}\end{array}$ & $\begin{array}{l}5.319 \\
(11.266)^{* * *}\end{array}$ \\
\hline$s($ Size beta $)$ & $\begin{array}{l}-0.002 \\
(-0.012)\end{array}$ & $\begin{array}{l}-0.006 \\
(-0.032)\end{array}$ & $\begin{array}{l}-0.017 \\
(-0.091)\end{array}$ & $\begin{array}{l}-0.015 \\
(-0.080)\end{array}$ & $\begin{array}{l}-0.011 \\
(-0.060)\end{array}$ & $\begin{array}{l}-0.013 \\
(-0.067)\end{array}$ \\
\hline$h$ (Value beta) & $\begin{array}{l}2.402 \\
(16.479)^{* * *}\end{array}$ & $\begin{array}{l}2.403 \\
(16.480)^{* * *}\end{array}$ & $\begin{array}{l}2.403 \\
(16.520)^{* * *}\end{array}$ & $\begin{array}{l}2.401 \\
(16.486)^{* * *}\end{array}$ & $\begin{array}{l}2.404 \\
(16.520)^{* * *}\end{array}$ & $\begin{array}{l}2.403 \\
(16.497)^{* * *}\end{array}$ \\
\hline$C L$ & $\begin{array}{l}1.569 \\
(5.196)^{* * *}\end{array}$ & $\begin{array}{l}1.516 \\
(4.980)^{* * *}\end{array}$ & $\begin{array}{l}1.877 \\
(6.113)^{* * *}\end{array}$ & $\begin{array}{l}2.367 \\
(7.051)^{* * *}\end{array}$ & $\begin{array}{l}1.943 \\
(6.312)^{* * *}\end{array}$ & $\begin{array}{l}2.373 \\
(7.058)^{* * *}\end{array}$ \\
\hline D_low & $\begin{array}{l}0.148 \\
(2.779)^{* * *}\end{array}$ & - & - & - & $\begin{array}{l}0.110 \\
(2.176)^{* *}\end{array}$ & - \\
\hline$C L \times D \_$low & - & $\begin{array}{l}0.466 \\
(2.487)^{* *}\end{array}$ & - & - & - & $\begin{array}{l}0.271 \\
(2.349)^{* *}\end{array}$ \\
\hline D_high & $\begin{array}{l}- \\
-\end{array}$ & $\begin{array}{l}- \\
-\end{array}$ & $\begin{array}{l}-0.276 \\
(-1.587)\end{array}$ & $\begin{array}{l}- \\
-\end{array}$ & $\begin{array}{l}-0.267 \\
(-1.555)\end{array}$ & - \\
\hline$C L \times D \_h i g h$ & $\begin{array}{l}- \\
-\end{array}$ & - & - & $\begin{array}{l}-0.220 \\
(-1.581)\end{array}$ & - & $\begin{array}{l}-1.057 \\
(-3.683)^{* * *}\end{array}$ \\
\hline$\overline{R^{2}}$ & 0.053 & 0.053 & 0.053 & 0.053 & 0.054 & 0.054 \\
\hline
\end{tabular}

$* 90 \%$ significance level $\quad * * 95 \%$ significance level $\quad * * * 99 \%$ significance level 
Table A3. (continued)

\section{Panel B. 27 Size-BE/ME-Corporate Liquidity portfolios}

In the first pass, portfolio excess returns are regressed on market, $S M B$ and $H M L$ factors. Each individual firm is assigned with betas of the portfolio that the firm belongs to. In the second pass, individual stocks' 1 year forward expected excess returns are regressed on factor betas from the first pass, corporate liquidity measures, liquidity constraint dummies or interaction terms of corporate liquidity and liquidity constraint dummies. Liquidity constraint dummies are defined as follows: D_low equals one if the time series average of individual firm's cash holdings falls below the bottom 30 percentile of the industry average level and zero otherwise. D high equals one if the time series average of individual firm's cash holdings is higher than the top 30 percentile of the industry average level and zero otherwise.

\begin{tabular}{|c|c|c|c|c|c|c|}
\hline & Model 8 & Model 9 & Model 10 & Model 11 & Model 12 & Model 13 \\
\hline constant & $\begin{array}{l}-5.134 \\
(-9.349)^{* * *}\end{array}$ & $\begin{array}{l}-5.012 \\
(-9.992)^{* * *}\end{array}$ & $\begin{array}{l}-4.923 \\
(-9.764)\end{array}$ & $\begin{array}{l}-5.129 \\
(-10.247)^{* * *}\end{array}$ & $\begin{array}{l}-4.922 \\
(-9.775)^{* * *}\end{array}$ & $\begin{array}{l}-5.119 \\
(-10.233)\end{array}$ \\
\hline$b$ (Market beta) & $\begin{array}{l}5.602 \\
(11.011)^{* * *}\end{array}$ & $\begin{array}{l}5.858 \\
(11.355)^{* * *}\end{array}$ & $\begin{array}{l}5.820 \\
(11.229)^{* * *}\end{array}$ & $\begin{array}{l}5.948 \\
(11.541)^{* * *}\end{array}$ & $\begin{array}{l}5.801 \\
(11.191)^{* * *}\end{array}$ & $\begin{array}{l}5.919 \\
(11.487)^{* * *}\end{array}$ \\
\hline$s$ (Size beta) & $\begin{array}{l}-0.083 \\
(-0.428)\end{array}$ & $\begin{array}{l}-0.103 \\
(-0.557)\end{array}$ & $\begin{array}{l}-0.080 \\
(-0.429)\end{array}$ & $\begin{array}{l}-0.099 \\
(-0.530)\end{array}$ & $\begin{array}{l}-0.085 \\
(-0.459)\end{array}$ & $\begin{array}{l}-0.101 \\
(-0.546)\end{array}$ \\
\hline$h$ (Value beta) & $\begin{array}{l}2.347 \\
(16.699)^{* * *}\end{array}$ & $\begin{array}{l}2.400 \\
(17.376)^{* * *}\end{array}$ & $\begin{array}{l}2.418 \\
(17.503)^{* * *}\end{array}$ & $\begin{array}{l}2.386 \\
(17.265)^{* * *}\end{array}$ & $\begin{array}{l}2.422 \\
(17.539)^{* * *}\end{array}$ & $\begin{array}{l}2.395 \\
(17.324)^{* * *}\end{array}$ \\
\hline$C L$ & $\begin{array}{l}1.145 \\
(3.293)^{* * *}\end{array}$ & $\begin{array}{l}1.584 \\
(3.830)^{* * *}\end{array}$ & $\begin{array}{l}1.156 \\
(2.492)^{* *}\end{array}$ & $\begin{array}{l}1.261 \\
(1.771)\end{array}$ & $\begin{array}{l}1.079 \\
(1.948)\end{array}$ * & $\begin{array}{l}1.266 \\
(2.486)^{* *}\end{array}$ \\
\hline D_low & $\begin{array}{l}0.183 \\
(1.832)^{*}\end{array}$ & - & - & - & $\begin{array}{l}0.132 \\
(1.896)\end{array}$ * & - \\
\hline$C L \times D \_l o w$ & - & $\begin{array}{l}0.149 \\
(1.641)\end{array}$ & $\begin{array}{l}- \\
-\end{array}$ & - & - & $\begin{array}{l}0.207 \\
(1.633)\end{array}$ \\
\hline D_high & - & - & $\begin{array}{l}-0.143 \\
(-1.097)\end{array}$ & - & $\begin{array}{l}-0.234 \\
(-2.100)^{* *}\end{array}$ & - \\
\hline$C L \times D \_h i g h$ & - & - & - & $\begin{array}{l}-0.253 \\
(-2.048)\end{array}$ & - & $\begin{array}{l}-0.285 \\
(-1.781)\end{array}$ \\
\hline$R^{2}$ & 0.045 & 0.046 & 0.047 & 0.047 & 0.048 & 0.048 \\
\hline
\end{tabular}

* 90\% significance level $\quad * * 95 \%$ significance level $\quad * * * 99 \%$ significance level 
Figure 1. Time Series of Corporate Liquidity

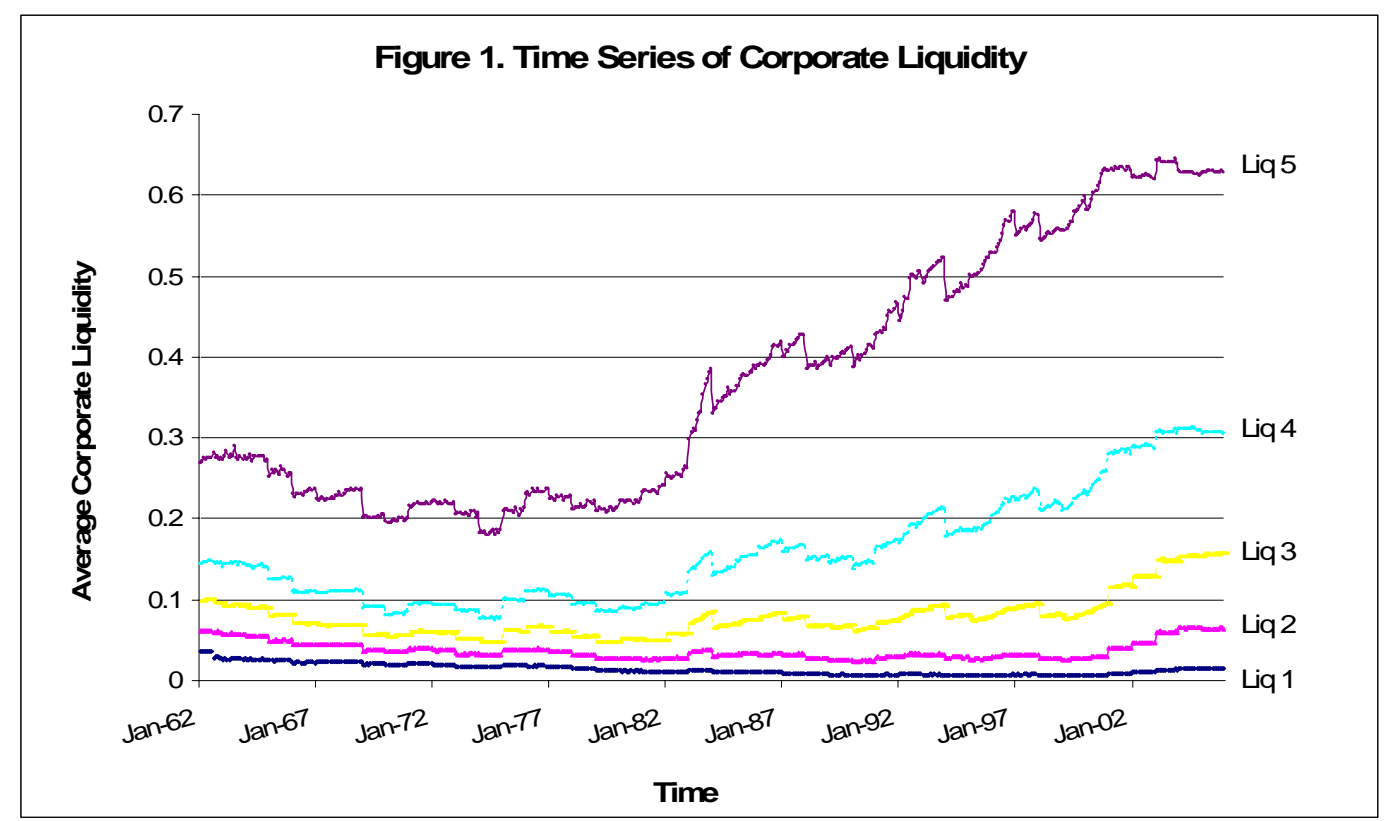

Figure 1 presents the time series of corporate liquidity for the sample period from 1962:01 to 2005:12. Each month all the firms are sorted into 5 portfolios based on corporate liquidity, and average corporate liquidity for the portfolio is calculated. Liq 1 represents the most illiquid portfolio, and Liq 5 represents the most liquid portfolio. 


\section{Chapter 2}

\section{Is Corporate Liquidity A Risk Factor?}

\section{Introduction}

Is corporate liquidity a risk factor? Does corporate liquidity contain information that can predict changes in the future investment opportunity set? Is corporate liquidity priced cross-sectionally or is it just an idiosyncratic risk? An extensive corporate finance literature investigates the determinants of corporate liquidity levels (Kim, Mauer and Sherman (1998) and Opler, Pinkowitz, Stulz and Williamson (1999)). These studies try to answer the question why firms keep liquid assets and examine the characteristics of firms associated with large cash holdings.

Although previous literature has established that cash holdings are important for firm investment and operation, and how various firm characteristics can affect the chosen asset liquidity level, it remains unclear whether cash holdings can enhance the value of firms, and to what extent aggregate cash holdings can be varying with equity returns. Some recent papers have started investigating the value of cash holdings at the individual firm level. Mikkelson and Partch (2003) find that, in the long run, firms retaining high cash reserves grow faster and undertake higher levels of investment, and they conclude that conservative financial policy can enhance operating performance. Pinkowitz and Williamson (2004) point out that cash holdings are more valuable for financially constrained companies and for firms with better growth opportunities, more volatile investment opportunities and a lower probability of financial distress. Faulkender and Wang (2006) adopt a methodology that examines the variation in excess equity returns as a measure of the marginal value of cash. They find that the market rewards firms holding internal cash with higher values, while the marginal market value of cash declines as the level of cash increases. They also find that on average, the market value of cash is higher for financially constrained firms than for those that are not constrained. Sibilkov (2005) finds that cash holdings are more valuable for financially constrained firms, partly because constrained firms use cash more efficiently to invest in more valuable projects than financially unconstrained firms. 
On the aggregate level, Greenwood (2004) uses the Federal Reserve Flow of Funds data to construct a measure of aggregate corporate liquidity, and finds that aggregate corporate liquidity is negatively related to future stock market returns. He argues that the evidence supports the active market timing theory, in which firms assess capital markets and accumulate cash when they appear overvalued since they do not have investment opportunities.

The asset pricing literature, on the other hand, tries to explain the asset returns with risk factors. The classical CAPM asserts that the only risk that the investors are compensated for bearing is the systematic risk that is associated with the stock market, which, however, turns out to be an empirical failure. Although Fama-French three-factor model can explain a substantial portion of cross-sectional stock return variations, it does not provide clear interpretations for the underlying risk factors that are proxied by $S M B$ and $H M L$. Meanwhile, there is an increasing body of microstructure literature that tries to analyze the behavior and formation of asset prices, in particular, the relationship between liquidity, information asymmetries and asset premia in the long run (see Easley and O’Hara (2003)).

The liquidity of a financial asset refers to the speed and ease with which the asset can be converted into cash (Glossary of Bodie, Kane and Marcus $4^{\text {th }}$ edition, 1999). The financial liquidity is typically considered a characteristic of an asset, but the market liquidity as an aggregate factor is also widely examined. Liquidity can be time-varying for the market as a whole, and therefore investors may be compensated for bearing this systematic risk. Chordia, Roll and Subrahmanyam (2000) argue that liquidity is not only an individual asset's attribute, but that there exists a commonality across assets based on underlying microstructure phenomena. The existence of commonality in liquidity raises the question whether trading cost shocks can be priced as a source of non-diversifiable risk. Pástor and Stambaugh (2003) examine whether the market-wide liquidity is priced in an asset pricing model. They find that liquidity risk is capable of explaining crosssectional expected return variation that is not captured by the Fama-French three factors. Stocks with higher "liquidity betas", tend to have higher expected returns on average, which provides support to the idea of aggregate liquidity as a factor. It is found that small stocks are less liquid and are more sensitive to aggregate liquidity. Acharya and Pedersen 
(2005) present a liquidity-adjusted capital asset pricing model in which the expected return of a financial asset is positively related to its expected illiquidity and its "net beta", which is proportional to the covariance of its return net of illiquidity costs with the net return on the market portfolio. The net beta can be further decomposed into the standard market beta and three betas representing different forms of liquidity risk associated with: (i) commonality in liquidity with market liquidity, $\operatorname{cov}\left(c^{i}, c^{M}\right)$ (Amihud and Mendelson (1986)); (ii) return sensitivity to market liquidity, $\operatorname{cov}\left(r^{i}, c^{M}\right)$ (Pástor and Stambaugh (2003)); and (iii) liquidity sensitivity to market returns, $\operatorname{cov}\left(c^{i}, r^{M}\right)$. The model also shows that liquidity moves together with contemporaneous returns and is able to predict expected future returns.

Holmström and Tirole (2001) make adjustments to asset pricing theory using a corporate finance approach. They develop an asset pricing model based on firms' desire to hoard financial slack. Collateralizable assets require a premium determined by the covariation between the asset's return and the marginal value of liquidity ${ }^{13}$ in different states. Risk neutral firms pay a premium on assets that can help them through the time when liquidity is in shortage. According to their model, asset prices are completely determined by corporate demand for liquidity.

This paper examines whether corporate liquidity can be a risk factor and can be priced in an asset pricing model. If aggregate corporate liquidity contains information about changes in future investment opportunities, it can be used as a factor to price equity returns. I construct a corporate liquidity factor $L M I$, which stands for "Liquid Minus Illiquid". $L M I$ is a zero-investment portfolio that is long stocks with the most liquid assets and short stocks with the least liquid assets.

I estimate the multifactor asset pricing models for the 25 portfolios constructed by independently sorting stocks by size and book-to-market equity, and the 27 portfolios constructed by independently sorting stocks by size, book-to-market equity and corporate liquidity. The explanatory power of $S M B$ strengthens while the loadings on $H M L$ are reduced substantially. The liquidity factor, $L M I$, captures the time-series variation of the excess stock returns, with loadings turning negative to positive from the most illiquid

\footnotetext{
${ }^{13}$ Liquidity in Holmström and Tirole (2001)'s paper refers to the aggregate value of financial securities that can transport wealth over time and back up future payments.
} 
portfolios to the most liquid portfolios and all the slopes for the liquid portfolios are highly significant. The opposite signs of the LMI loadings suggest that the corporate liquidity factor loads on illiquid firms and liquid firms in an opposite way. On average, liquidity loads negatively on expected returns for the most illiquid firms but positively on more liquid firms. The Gibbons-Ross-Shanken (GRS) test fails to reject the null hypothesis that intercepts from the time-series regression are jointly indistinguishable from zero and the four-factor model well explains the common variations in a crosssection of excess stock returns.

In addition, when CAPM, Fama-French three-factor model and the four-factor model are estimated for 10 corporate liquidity portfolios, I find that market betas are higher for liquid portfolios than illiquid ones, and loadings on $L M I$ increase along with corporate liquidity, which supports that corporate liquidity is a risk factor.

Furthermore, multifactor regressions are performed for the 25 portfolios constructed by subsequently sorting all the firms by size and corporate liquidity, and by BE/ME and corporate liquidity. The empirical results provide more support for the existence of corporate liquidity effect.

There is no clear evidence that the LMI factor co-moves with macroeconomic conditions. The corporate liquidity premia are not quite sensitive to changes in macroeconomic conditions or monetary policy. Furthermore, when examining the relationship between companies' performance and macroeconomic conditions, the regression results show that liquid firms are actually less cyclical than illiquid firms.

Moreover, I find that the corporate liquidity beta is higher during recessions, and it seems that the corporate liquidity beta is more sensitive to changes in macroeconomic variables for liquid firms than illiquid firms. These results support the idea that companies pile up cash and cash equivalents for precautionary purposes. Firms who are more sensitive to the less favorable economic conditions will increase their liquid asset holdings when they forecast an economic downturn. Those firms are riskier because of their higher sensitivity to changes in macroeconomic condition.

Finally, I examine whether the corporate liquidity beta of financially constrained firms and unconstrained firms responds differently to the changes in a macroeconomic variable. The corporate liquidity beta of financially constrained companies is more sensitive to 
economic news. These empirical results support the view that firms stock liquidity because they are financially constrained and therefore have greater exposure to macroeconomic fluctuations.

The remaining of the paper is organized as follows. Section II formulates the hypotheses to be tested. Section III briefly discussed the data and variables. Section IV presents the empirical results. Section V investigates the relationship between corporate liquidity and macroeconomic variables. Section VI concludes.

\section{Hypotheses}

Variability in a security's total returns that is directly associated with overall movements in the general market or economy is systematic risk. Systematic risk is not diversifiable. Expected returns are higher for stocks with priced higher systematic risk because investors require more compensation for higher non diversifiable risk. Chapter 1 discusses the corporate liquidity as a source of idiosyncratic risk, and empirical evidence shows that equity returns are positively related to corporate liquidity, even if size and value effect have been controlled for. Furthermore, Chapter 1 shows that firm market betas have a direct relationship with corporate liquidity, and the regression results support the precautionary saving motive for companies and a signaling effect of corporate liquidity. These findings lead to another interesting question: Can corporate liquidity be associated with systematic risk, and why?

Let's assume a dynamic world where the Intertemporal Capital Asset Pricing Model (ICAPM) of Merton $(1973,1990)$ is true. Different from the static asset pricing models where the distribution of asset returns does not change over time, Merton's ICAPM is derived in an environment with time-varying investment opportunities.

In Merton's model, a vector of state variables represents changes in investment opportunities over time. In a simple discrete time version of ICAPM ${ }^{14}$, the marginal utility given a change in wealth during a certain period can be expressed as a linear combination of these uncorrelated state variables. Therefore, if the return of each asset in

\footnotetext{
${ }^{14}$ The original Merton's ICAPM applies continuous time stochastic dynamic programming approach, where returns are assumed to follow a Brownian motion process. Constantinides (1989) discusses a discrete-time analog of Merton's ICAPM, in which returns are multivariate normally distributed.
} 
one's wealth portfolio can be represented by a set of portfolio returns which are correlated with those state variables, we can use the portfolio returns as factors to mimic the state variables and hence the future investment opportunities. Merton's ICAPM does not specify what these factors would be, rather, it provides a very general framework in which any variable that can explain cross sectional asset returns can be considered as a factor that proxies the varying investment opportunities.

If only the firms observe their own changes in real investment opportunities, then it may well be that aggregate corporate liquidity is the best publicly available measure for expected future returns, which in turn summarizes the available investment opportunities. So effectively then we have a Merton model in which the aggregate liquidity is the state variable that serves as a second factor in addition to the market factor. $S M B$ and $H M L$ may capture other state variables representing different aspects of investment opportunities.

If firms accumulate liquid assets because managers have better information about changes in future investment opportunities, aggregate corporate liquidity may signal this information, measure expected equity returns, hence can be used as an aggregate factor. Given that investors are rational, they will understand that liquid asset piling up signals changes in a set of investment opportunities, which may represent potential risks for investors. Investors therefore will require higher equity returns in order to bear the risks from the varying investment opportunities and expected returns. In general, aggregate corporate liquidity can proxy a state variable and can be used as a factor to model the equity return variation. A higher level of aggregate corporate liquidity may indicate riskier investment opportunities in the future. The above analysis leads to the first hypothesis.

Hypothesis 1: If corporate liquidity contains information about changes in investment opportunities which may represent a source of risk, aggregate corporate liquidity serves as a factor and requires a positive risk premium.

Fama-French three-factor model adds two factors $S M B$ and $H M L$ to CAPM to reflect a portfolio's exposure to these two classes. Fama and French see high returns as a reward 
for taking on higher risk; in particular if returns increase with the book/price ratio, then stocks with a high book-to-market equity ratio must be riskier than an average stock. They argue high book-to-market equity could mean a stock is "distressed", temporarily selling at low prices because future earnings look doubtful. Fama and French interpret $H M L$ as a risk factor measuring financial distress. Given a Merton environment, aggregate corporate liquidity may serve as a state variable and capture part of the financial distress risk. Here I construct a factor LMI (Liquid Minus Illiquid), which represents changes in investment opportunities, including likely changes in distress risk as firms stock more cash if they anticipate financial distress. Similar arguments may apply for the size factor $S M L$. This leads to the second hypothesis.

Hypothesis 2: If LMI is a systematic risk factor, the explanatory power of Fama-French distress factors SMB and/or HML should decrease when LMI is included in the multifactor regression.

On an aggregate level, if the corporate liquidity signals the firms' risk, then the equity returns of more liquid and more illiquid firms should respond differently to changes in macroeconomic conditions. If holding more corporate liquidity implies higher risk of the firm, the proxy for the systematic risk - the corporate liquidity factor $L M I$ - will vary together with the macroeconomic changes.

Hypothesis 3: If the corporate liquidity factor is a proxy for systematic risk, it should forecast future returns and should be contemporaneously correlated with leading indicators of the business cycle.

Asset pricing theories indicate the sources of systematic risk come from the correlation between equity returns and the changes in macroeconomics. In the long run, equity returns are determined by fundamentals. If liquid firms store financial slack because they expect riskier future cash flows and poorer operating performance after negative shocks to the aggregate economy, then corporate liquidity signals a systematic risk. In other words, companies with a higher level of financial reserves may experience poorer 
fundamentals during economic downturns. For example, if a firm expects the economy is slowing down, to better prepare itself to get through the next recession, the firm will stock more liquid assets as a cushion. When macroeconomic condition becomes less favorable, the firm's sales decrease, profits plummet and cash flows are more volatile, but these liquid firms may probably be safe since they have enough cash as a buffer. On the contrary, for those companies whose fundamentals are less sensitive to the worse economic condition, they do not need to stock more liquidity. What we observe maybe that on the aggregate level, liquid firms' business fundamentals maybe more cyclical than less liquid firms. If corporate liquidity signals adverse changes in investment opportunities and represents a source of systematic risk, we would expect that business fundamentals of firms with more corporate liquidity are more closely tied to the business cycle. This leads to hypothesis 4 as follows:

Hypothesis 4: If firms with higher corporate liquidity are riskier, then fundamental business activities of more liquid firms should be more cyclical than those of illiquid firms.

The first two hypotheses are tested in Section IV and the last two hypotheses are tested in Section V.

\section{Data}

To test whether corporate liquidity is a risk factor, I construct a sample of firms for the empirical tests by merging the COMPUSTAT annual files and the stock and indices databases from the Center for Research in Security Prices (CRSP) for the period 1962:1 to 2005:12. The CRSP returns cover NYSE, AMEX and NASDAQ stocks. Financial firms with Standard Industrial Classification (SIC) codes between 6000 and 6999 are excluded from the sample because they usually hold substantial inventories of marketable securities, which are included in corporate liquidity measure. Transportation and public utility firms with SIC codes between 4000 and 4999 are excluded as well because cash holdings of these firms are subject to regulatory supervision. 
Firms' monthly stock returns are taken from CRSP. Market excess returns are downloaded from Kenneth French's webpage. ${ }^{15}$ Monthly risk free interest rates are one year Treasury bill rates obtained from Kenneth French's webpage.

Corporate liquidity is measured by the ratio of cash and marketable securities (Compustat item \#1) to total assets (Compustat item \#6):

$$
C L_{i t}=\frac{\text { Cash }_{i t}}{\text { Total Assets }_{i t}}
$$

where Cash $_{i t}$ includes the following items: bank drafts, cash, checks (cashiers or certified), demand certificates of deposit, demand deposits, letters of credit and money orders. It also includes government and other marketable securities and time deposits, which are usually considered as cash equivalents. A firm with a high cash-to-asset ratio has more liquid assets to serve interest payments, and therefore postpone bankruptcy and reduce financial distress; or has more freedom to invest in profitable projects without incurring transaction costs.

Firm size is measured by the market value of equity, which equals the stock price of the stock at the end of June of year $t$ times the total shares outstanding. Following Fama and French (1992), the book-to-market equity ratio (BE/ME) at time $t$ is calculated by dividing book equity (BE) at the fiscal ending of year $t-1$ by market equity (ME) at December of year $t-1$. The book-to-market equity ratios at time $t$ are matched by the stock returns from July of year $t$ to June of year $t+1$. Therefore there is at least a 6 months' lag between accounting data and market data in order for the firms' accounting information to be released and understood by the public. The book value of firm equity is defined as in Cohen, Polk and Vuolteenaho (2003). ${ }^{16}$ Leverage is calculated as (long-term debt +

\footnotetext{
${ }^{15} \mathrm{http}: / /$ mba.tuck.dartmouth.edu/pages/faculty/ken.french

${ }^{16}$ Book equity is defined as the stockholders' equity, plus balance sheet deferred taxes (Compustat item \#74) and investment tax credit (Compustat item \#208), plus postretirement benefit liabilities (Compustat item \#330), minus the book value of preferred stock. Depending on availability, they measure the book value of preferred stock by the order of redemption (Compustat item \#56), liquidation (Compustat item $\# 10$ ), or par value (Compustat item \#130). Stockholders' equity is measured by Compustat item \#216 or the book value of common equity (Compustat item \#60), plus he par value of preferred stock, or the book value of assets (Compustat item \#6) minus total liabilities (Compustat item \#181).
} 
short-term debt)/(book value of assets) ${ }^{17}$ following Opler, Pinkowitz, Stulz and Williamson (1999).

I use three alternative financial constraint measures suggested by the literature. (1) Asset size. Gilchrist and Himmelberg (1995) argue that smaller firms are more likely to be subject to financial constraint because they are usually young and less well known, hence have difficulty in accessing external capital market. (2) KZ index, which is a linear index of firm financial constraints constructed based on Kaplan and Zingales (1997). Following Lamont, Polk and Saá-Requejo (2001), the KZ index is calculated using the following equation ${ }^{18}$ :

$$
\begin{aligned}
\text { KZindex }= & -1.002 \times \text { CashFlow }+0.283 \times Q+3.319 \times \text { Leverage } \\
& -39.368 \times \text { Dividends }-1.315 \times \text { CashHoldings }
\end{aligned}
$$

The $\mathrm{KZ}$ index is a linear combination of five accounting measures and it measures the likelihood of financial constraint. The higher the KZ index, the less cash flow, the higher leverage and lower dividend distribution and therefore the more constrained the firm is. (3) Dividend payout, which is calculated as the ratio of total dividends distributions to operating income. Fazzari et al. (1988) finds that firms with financial constraints distribute significantly less dividends.

Firm specific characteristics and dependent variables are trimmed at the 1 percent tails of the entire sample. Firms with negative total assets, book value and market value of equity are deleted from the sample. I also delete firms with less than 36 monthly observations. There are 4525 firms and 528 months in the sample. Table 1 presents the summary statistics.

Macroeconomic variables include GDP, Industrial production $(I P)$ index, the NBER recession dummy, the federal funds rate $(F F R)$ and the $C P I$. Macroeconomic variables are obtained from websites of the Bureau of Labor Statistics, the Federal Reserve Board (Statistical Release H.15), the Federal Reserve Bank of St. Louis, and the NBER.

\footnotetext{
${ }^{17}$ Long term debt is measured by Compustat item $\# 9$ and short term debt is measured by Compustat item \#5.

${ }^{18}$ Tobin's Q, calculated as the market value of assets divided by book assets. Cash flow, dividends and cash holdings are all standardized by total capital
} 


\section{Empirical Results}

\section{IV.1. Does the Corporate Liquidity Factor Have a Risk Premium?}

Chapter 1 finds that firms with higher corporate liquidity have higher expected equity returns as well as market betas. One reasonable interpretation is that corporate liquidity contains some private information and can proxy for the underlying unobservable risks. In order to investigate the pricing implication of corporate liquidity, first we need to examine whether corporate liquidity as a factor entails positive risk premium.

Table 1 summarizes the basic statistics for various company characteristics. Panel A shows the distribution of these variables. Panel B displays the average premium on FamaFrench risk factors as well as the corporate liquidity factor $L M I$ (Liquid Minus Illiquid). $L M I$ is a zero-investment portfolio that is long stocks with relatively highly liquid asset holding and short stocks with the least liquid asset holdings. It is constructed by taking the difference between the average returns of the top 30 percent liquid stocks and the bottom 30 percent illiquid stocks. It can be shown that the average value of $L M I$ is 0.753 percent per month (9.024 percent per year), with the t-value equal to 5.142 , both statistically and economically significant.

The corporate liquidity factor is further decomposed into two sub liquidity factors $L M I_{\text {med_low }}$ and $L M I_{\text {high_low }}$, which stand for median minus low and high minus low respectively. $L M I_{\text {med_low } t}$ is a zero-investment portfolio that is long stocks with medium level liquid asset and short stocks with the least liquid asset holdings. It is constructed by taking the difference between the average returns of the medium 40 percentile liquidity level stocks and the bottom 30 percentile illiquid stocks. $L M I_{\text {high_med }}$ is a zero-investment portfolio that is long stocks with the highest corporate liquidity and short stocks with medium liquid asset holdings. It is constructed by taking the difference between the average returns of the top 30 percentile liquid stocks and the medium 40 percentile liquidity stocks. These two sub liquidity factors are constructed in order to examine whether the importance of corporate liquidity factor varies with the degree of corporate liquidity itself. In another word, we would like to find out whether corporate liquidity effect is stronger for higher cash holding firms or lower cash holding firms. $L M I_{\text {med_low } t}$ tries to capture the effect of liquidity constraints impose on the expected equity returns

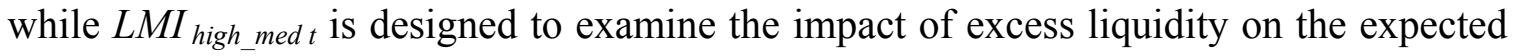


equity returns. It turns out that the corporate liquidity premium is positive for both sub liquidity factors, but it seems that $L M I_{\text {high_med }}$ requires a much higher premium than $L M I$ med_low $t_{\text {. }}$ The monthly mean return is a highly significant 0.538 percent for $L M I_{\text {high }}$ med ${ }_{\text {t }}$, while it is only 0.285 percent for $L M I_{\text {med_low }}$.

\section{IV.2. Does Corporate Liquidity Contain the Same Information As SMB and HML?}

We have observed strong evidence of the existence of positive corporate liquidity premium, which is greater for relatively more liquid firms than illiquid firms. If corporate liquidity contains information that represents investment opportunities over time, then the aggregate liquidity may serve as a factor and moves together with equity returns. Does corporate liquidity contain different information with the commonly accepted market, size and value factor? Panel C of Table 1 presents the Pearson's correlation matrix between various factors. It can be shown that aggregate corporate liquidity factor is positively correlated to market factor and size factor, but strongly negatively correlated to the value factor. Fama and French (1996) suggest that financially distressed firms are more sensitive to the shocks to future earning streams. Fama and French (1995) and Liew and Vassalou (2000) find that distress risk has a stronger effect during recessions. In a word, value firms are riskier during a recession. On the other hand, illiquid firms are riskier in recessions since they have less financial slack as a cushion while sales may drop, cash flows are expected to be low and external financing becomes more difficult. This gives a negative correlation between $H M L$ and $L M I$, and is consistent with the issue that when a recession is anticipated illiquid firms will start hoarding more cash.

In addition, In order to formally investigate whether the corporate liquidity factor shares some similar information with the well-known CAPM and Fama-French three risk factors or if it contains different information, we estimate the following time series regressions:

$$
\begin{aligned}
& L M I_{t}=\alpha+\beta M K T_{t}+\varepsilon_{t} \\
& L M I_{t}=\alpha+\beta M K T_{t}+s S M B_{t}+h H M L_{t}+\varepsilon_{t},
\end{aligned}
$$

where $L M I_{t}$ is the overall corporate liquidity factor. $M K T, S M B$ and $H M L$ are the three Fama-French factors. In addition, we also run the time series regression for the two 
decomposed sub corporate liquidity factors: $L M I_{\text {med_low }}$ and $L M I_{\text {high }}$ med. Here we have three things to look at. First, if the coefficients on $M K T S M B$ and $H M L$ are significant, then we can conclude that our corporate liquidity factor does share some information with the size and value factor, and that the corporate liquidity risk premium moves together with the common risk factors. Otherwise, $L M I$ serves as a risk factor that is different from $M K T, S M B$ and $H M L$. Second, if the other three common factors have priced the corporate liquidity factor itself an excess return correctly, we should expect an insignificant intercept. Third, we can tell whether the $L M I$ can serve as an additional source to explain the stock return variations by looking at the $R$-squared. If the $R$-squared is low, then return variation in $L M I$ is not well explained by the above asset pricing models, and it may contain different information other than that in existing factors.

Table 2 presents the regression results of the above models. Column 1 shows the results for the CAPM. The corporate liquidity factor has a significant market beta equal to 0.201 , indicating that liquid firms have higher betas than illiquid firms. The intercept is statistically significant, meaning that the corporate liquidity factor is mispriced by the Capital Asset Pricing Model by 66.3 basis points monthly. The $R$-squared is only 7 percent, which says that market factor can only explain a small portion of the information contained in the LMI.

Column 2 uses the Fama-French three-factor model. The market factor loads positively on $L M I$. The size factor $S M B$ has a significant positive loading 0.205 regressed on the overall corporate liquidity factor $L M I$. The value factor $H M L$ loads significantly but negatively, with a loading of -0.685 , which means that liquid firms have lower $H M L$ betas. The overall corporate liquidity factor has a strong relationship with the size and value factors, indicating that the relative performance of liquid firms versus illiquid firms tends to move together with the size and value portfolios. The constant alpha is again highly significant, which means the corporate liquidity factor is still mispriced by the Fama-French three-factor model. The adjusted $R$-squared is 0.43 , implying a substantial explanatory power of the regression. To some extent, corporate liquidity factor shares common information with size and value factor, however still more than half of the return variations in $L M I$ cannot be accounted for. 
As the dependent variable is changed to $L M I_{\text {med_low }}$ and $L M I_{\text {high_med }}$ respectively, we can see that the $S M B$ loading becomes is reduced substantially for $L M I_{\text {med_low }}$ regression and remains about the same for $L M I_{\text {high } \_ \text {med. }}$. The loadings on $H M L$ are always negative. However, the magnitude of $H M L$ loading decreases when it is regressed on $L M I_{\text {med_low }}$.

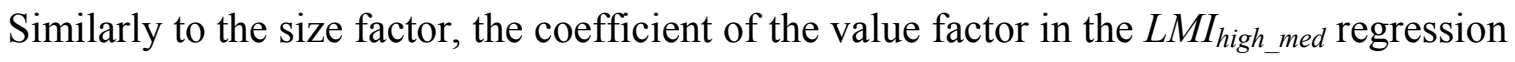
is similar in magnitude than that in the $L M I$ regression. Finally, the $R$-squared for the $L M I_{\text {med_low }}$ regression drops to only 0.30 , while it remains roughly the same for the $L M I_{\text {high } m e d}$ regression. These results suggest that corporate liquidity effect seems to concentrate in firms with more liquid assets; and the corporate liquidity factor seems to be more important and shares more common information with size and value factor for those firms with more corporate liquidity.

\section{IV.3. Multifactor Asset Pricing Models on 25 Size-BE/ME portfolios}

To further explore the asset pricing implications of corporate liquidity, together with established factors such as market, size and $\mathrm{BE} / \mathrm{ME}$, I consider the multifactor asset pricing models, in particular, the Fama-French three-factor model, and test whether the fourth factor - a corporate liquidity factor - can replace the size or value factor, or help improve the overall performance. Following Fama and French (1993), I adopt a timeseries approach, in which asset-pricing tests consist of two parts. First, it needs to be shown that the four factors - market, size, value and corporate liquidity - capture the common variation in stock returns and are indeed risk factors. This can be established by looking at the slopes and $R$-squareds of the regression directly. Then, intercepts of the time-series stock return regressions need to be examined to test whether the risk factors explain the cross-section of average stock returns. The test assets for both Fama-French three-factor model and the multifactor asset pricing model with the additional corporate liquidity factor in this section are the 25 portfolios formed from the intersection of independent sorting of all the stocks into five size and five book-to-market equity portfolios. 


\section{IV.3.1 The Fama-French Three-Factor Model}

Fama-French three-factor model is performed first as a benchmark. In this time-series regression, portfolio excess returns are regressed on the three factors that are considered to be able to explain the common variation of stock returns. The model is as follows:

$$
R_{t}^{i}-R F_{t}=a^{i}+b^{i} M K T_{t}+s^{i} S M B_{t}+h^{i} H M L_{t}+e_{t}
$$

$M K T_{t}$ is the return on the market factor, which equals the stock market return minus the risk free rate. $S M B_{t}$ proxies the size risk factor, which is calculated as the difference between the average returns on small- and big-stock portfolios with same book-to-market equity in the my sample. $H M L_{t}$ stands for the risk factor in returns related to book-tomarket equity; it is defined as the difference between the simple average returns of the top 30 percent $\mathrm{BE} / \mathrm{ME}$ portfolios (high $\mathrm{BE} / \mathrm{ME}$ ) and the bottom 30 percent $\mathrm{BE} / \mathrm{ME}$ portfolios (low BE/ME) with same size. The size factor is the zero-investment portfolio that longs small firms and shorts large firms while the book-to-market factor is the zeroinvestment portfolio that longs high book-to-market stocks and shorts low book-tomarket stocks. Panel B of Table 1 shows that the average value of the market factor is 0.448 percent per month, which is both statistically and economically significant. The size factor $S M B$ produces an average monthly premium equal to 0.229 percent. The average value of $H M L$ is 0.464 percent, with a $t$-value equal to 2.896 . The slopes $b, s$ and $h$ are the factor loadings which measure the impact of these factors on the portfolio returns. If the factors can capture common variation in stock returns and establish themselves as risk factors, then we should observe significant slopes from the time-series regression. The intercept, $a$, is the mis-pricing residual, or called alpha. If the above explanatory factors are good proxies for common risk factors or underlying state variables, according to the multifactor asset-pricing models of Merton (1973) and Ross (1976a), a simple test of whether and how well the average return premia for the risk factors can explain the cross sectional variation of average stock returns is to examine the significance of the intercepts in the time-series regression. We should observe intercepts that are insignificantly different from zero if the explanatory factors are sufficient to describe the cross-section of average stock returns. 
Table 3a presents the results of the Fama-French three-factor model. The excess returns on the market portfolio capture a substantial amount of cross-sectional variation of stock returns. The market betas for stocks are all more than 9.10 standard errors from 0 . $S M B$ contributes significantly to explain stock returns variations over time. Consistent with Fama-French (1993), the coefficients of $S M B$ are negatively related with size. In all the book-to-market quintiles except the highest $\mathrm{BE} / \mathrm{ME}$ portfolio, the slope of $S M B$ decreases as size increases. The slopes of $H M L$, on the other hand, decline with the bookto-market ratio. The loadings on $H M L$ do not seem to increase from lower- to higherbook-to-market quintiles. Most of the slopes for the value factor are highly significant. However, it seems that the sensitivities of stock returns to the value factor are not as strong as to the size factor in this sample. The R-squareds are all above 0.68 , suggesting the three-factor model does a good job in capturing the common variation in stock returns and therefore the three stock returns: $M K T, S M B$ and $H M L$ are risk factors.

The second test focuses on the intercepts of the time-series regressions. It is apparent that in Table 3a, all the 25 intercepts are not statistically distinguishable from 0 except for the two extreme portfolios: the one with the smallest size and highest book-to-market equity and the one with the largest size and the smallest book-to-market equity. Time series regression intercepts that are not significantly different from zero suggest that the set of premiums that are associated with the three risk factors is sufficient to absorb the time-series variation and explain the cross-section of average stock returns. The absolute value of alpha is from 0.01 percent to 0.72 percent per month, which is an annual abnormal return of 0.12 percent to 8.64 percent. The formal test on the hypothesis of joint zero intercepts is the F-statistic of Gibbons, Ross and Shanken (1989), which tests whether the intercepts from the time-series regression of the excess stock returns on the risk factor return premiums are jointly significantly different from zero. The F-statistic for GRS test is 2.95 , greater than the critical value 1.53 at the 95 percent level. Contrary to the insignificant intercepts for the 25 portfolios individually, the null hypothesis that the intercepts are jointly insignificantly different from zero can be rejected with a $p$-value equal to 0.000 .

As a comparison, Table $3 \mathrm{~b}$ presents the regression results of the excess returns on market factor and the corporate liquidity factor for the 25 size-BE/ME portfolios. The 
loadings on the $L M I$ are all positive and significant for 20 out of the 25 portfolios. Furthermore, it seems that $L M I$ loadings are higher for big firms and value firms. The $R$ squareds are decent with only two factors, which is especially true for big firms and value firms. For portfolios with the highest BE/ME ratio, the market factor and $L M I$ captures at least 62 percent of the return variations.

\section{IV.3.2 The Four-Factor Model}

Since the intent of this study is to examine whether the firm's liquidity helps explain the cross-section of stock returns and whether this liquidity can serve as a risk factor and be priced, a four-factor model is estimated in this section as follows:

$$
R_{t}^{i}-R F_{t}=a^{i}+b^{i} M K T_{t}+s^{i} S M B_{t}+h^{i} H M L_{t}+c^{i} L M I_{t}+e_{t}
$$

The first three factors are the same as in the Fama-French three-factor model. The new factor, $L M I_{t}$, stands for "Liquid Minus Illiquid". If firms' cash positions can actually capture common variation in stock returns, we should expect the factor loadings of $L M I$ to be significant. If all the price information contained in $H M L$ is related to the firms' liquidity position and $L M I$ can replace the role of the value factor, we should be able to see the slopes of $H M L$ to lose significance. Finally, if the corporate liquidity factor contains different risk information than $H M L$ but helps explain the cross-sectional variation of stock returns, it can be expected that the R-squareds would be increased greatly and the intercepts should collapse more closely to zero. Panel $\mathrm{C}$ of Table 1 shows that the correlation between $H M L$ and $L M I$ is -0.629 , which is statistically significant. $L M I$ is positively correlated with $M K T$ and $S M B$.

The time-series regression results of the four-factor model are exhibited in Table 4. With the inclusion of the corporate liquidity factor $L M I$, market betas still as in Table 3a, barely change for most of the size-BE/ME portfolios. In general, the market factor still captures a great deal of the time-series variations of stock returns. The slopes of the size factor $S M B$ increase a little bit for each of the 25 portfolios, and the $t$-statistics increase as well. When the liquidity factor $L M I$ is included in the time-series regression, the role of the size factor is strengthened, although to a limited extent. The loadings of $H M L$ have been decreased for all the 25 portfolios, with a significant magnitude. For the 14 
portfolios with smaller size and higher book-to-market equity, the slopes of $H M L$ are reduced by more than 50 percent with the inclusion of $L M I$.

Table 4 shows that 23 out of the 25 portfolios have significant slopes for the corporate liquidity factor $L M I$. There is no clear monotonic pattern of how the $L M I$ loadings vary across size and BE/ME sorted portfolios. Relatively speaking, it seems that the excess stock returns are more sensitive to the corporate liquidity factor for growth firms and large firms, which may distinguish small and value firms. $R$-squareds do not improve much when $L M I$ is added in the regression. The corporate liquidity factor improves the performance by capturing additional time-series stock returns variations of all the 25 portfolios.

The intercepts actually move away from zero, with higher absolute values of the $t$ statistic. Although for 22 of the portfolios, the intercepts are still statistically indistinguishable from zero, the larger magnitude of the intercepts shows that the fourfactor model does not very well explain the cross-sectional variations of the stock returns for the 25 portfolios. The average value of alphas is 0.30 percent per month, which is an annual 3.60 percent. The GRS test also rejects the null hypothesis that the intercepts are jointly zero with an $F$ statistic equal to 3.02 and $p$-value of 0.000 .

\section{IV.4. Multifactor Asset Pricing Models on 27 Size-BE/ME-Corporate Liquidity \\ Portfolios}

The previous section performs the multifactor asset pricing models on Fama-French 25 portfolios, formed by independently sorting all stocks by size and book-to-market equity. In order to obtain the maximum dispersion against all explanatory variables, I run the multifactor models on a different set of 27 size-BE/ME-corporate liquidity portfolios. To construct the 27 portfolios, I perform a three-way independent sort. All the stocks in my sample are sorted into three portfolios according to size. Then they are sorted in three book-to-market equity portfolios. Finally, they are sorted in three portfolios according to

corporate liquidity. Twenty-seven equally weighted portfolios are formed from the intersection of the three independent sortings. Table 5 provides the summary statistics of the 27 portfolios. It can be shown that holding size and BE/ME constant, liquid firms have higher equity returns, lower leverage, higher cash flow volatility and higher market 
beta on average. In addition, within each size-BE/ME group, liquid firms tend to be larger firms and value firms.

\section{IV.4.1 The Fama-French Three-Factor Model}

Table 6 shows that the three factors capture the time-series variation of returns of the 27 test portfolios, further confirming that market, size and value factors are risk factors. The slopes of excess market returns are highly significant for all the 27 portfolios, with the lowest $t$-statistics 10.70 . The loadings on $S M B$ are significant for all the portfolios. For each of the corporate liquidity and book-to-market equity tertiles, it can be shown that the slopes of $S M B$ decline monotonically from the smallest portfolios to the biggest portfolios. Slopes of $S M B$ vary substantially across the 27 portfolios and seem to successfully capture the shared variation across stocks that are related to size. The slopes of $H M L$ are significant at a significance level of at least 90 percent for 26 out of the 27 portfolios. The slopes of the $H M L$ factor are related to book-to-market equity as well. For almost all the corporate liquidity and size portfolios, the slope coefficients increase monotonically as the book-to-market equity increases. $R$-squareds for the 27 time-series regressions are all above 0.61 .

Table 6 also shows that the estimated intercepts are not distinguishable from zero for 21 portfolios at a 0.95 significance level. Moreover, intercepts do not exhibit any pattern that is related to size and book-to-market equity, which supports the argument that the average premia for the three risk factors well explain the cross-section of the average excess stock returns. A formal GRS test is performed to test the null hypothesis that the time-series regression intercepts are jointly equal to zero. The GRS $F$ statistic for the three-factor model is 1.43 and the p-value is 0.09 . Hence we cannot reject the null hypothesis at a 0.95 significance level. By testing whether the intercepts are significantly different from zero, we are able to draw the conclusion that the three factor model is able to interpret the common variation of stock returns cross-sectionally.

\section{IV.4.2 The Four-Factor Model}

The four-factor model is estimated by performing a time-series regression of excess stock returns on the 27 size, book-to-market equity and corporate liquidity sorted 
portfolios on the Fama-French three factors and the corporate liquidity factor. The regression results are presented in Table 7.

According to Table 7, all the slopes of the market factor are highly significant, with the minimum $t$ statistic equal to 10.66 . The loadings on the market factor do not change much compared to the three-factor model, hence the inclusion of $L M I$ does not weaken the explanatory power of $M K T$. In addition, the sensitivities of excess stock returns on $S M B$ do seem to be higher in the four-factor model. Compared to $M K T$ factor and $S M B$ factor, changes in the loadings on $H M L$ are much more pronounced. Specifically, for 25 of the 27 portfolios, the loadings on $H M L$ are reduced substantially when $L M I$ is included in the regression. Relative to the regressions using the three factors, the four-factor model implies that excess stock returns are less sensitive to the value factor. The corporate liquidity factor may contain some information similar to the $H M L$ and hence decreases the loadings on the value factor. Both the slopes of the $S M B$ factor and the $H M L$ factor remain to be related with size and book-to-market equity respectively. For all the corporate liquidity and book-to-market equity portfolios, slopes of $S M B$ decline along with the increase in size. For all the corporate liquidity and size portfolios, slopes of $H M L$ increase with the book-to-market equity. Size and value factors in the four-factor models capture the cross-sectional variation of the stock returns that are associated with size and book-to-market.

As expected, the slopes of the liquidity factor $L M I$ are negative for almost all the illiquid portfolios but are all positive for the most liquid portfolios. $L M I$ decreases the average returns on stocks of the firms holding less cash and increases the average returns on stocks of the firms holding more cash. For those firms that have a low level of liquid assets, being more illiquid is quite risky since these firms might not be able to service their debts, survive through the next economic downturn, or take advantage of new profitable investment opportunities. Therefore investors may require a higher rate of return in order to be compensated for bearing the financial distress risks of holding illiquid firms' stocks. On the other hand, for those companies that already fall into the most liquid portfolios in terms of cash holdings, hoarding extra liquid assets might send negative signals to the market, indicating that the firms do not have adequate growth opportunities and use cash inefficiently. An alternative interpretation is that holding 
excessive corporate liquidity may indicate that the firms have volatile cash flows as well as higher underlying risks, sending negative signals to the stock markets. Investors hence require higher returns on those already-liquid firms with even more liquid assets holdings. Although not all the slope coefficients in the illiquid and medium liquidity portfolios are significant, almost all the slopes in the liquid portfolios are highly significant, implying that the sensitivities of stock returns to the liquidity factor are the strongest for firms with a high level of liquid assets.

Furthermore, the loadings of the $L M I$ factor are related to the corporate liquidity. Within each size and book-to-market equity portfolio, the slopes of $L M I$ increase from the most illiquid portfolios to the most liquid portfolios, in most of the cases, from negative to positive. This indicates that the $L M I$ does a great job in capturing the common variations that cannot be explained by size and value factor in a cross-section of the stocks. Table 7 provides evidence for the existence of a corporate liquidity factor because liquid firms have stock returns move together over time while illiquid firm's returns covary. R-squareds increase moderately for most of the portfolios, with the greatest improvement in the nine portfolios that are most liquid.

Table 7 shows that most of the intercepts of the time-series regressions on the 27 portfolios are not significantly different from zero. The intercepts do not show any obvious pattern across size, book-to-market and corporate liquidity portfolios. The insignificant and similar intercepts say that return premiums associated with the four factors are sufficient to absorb the time-series variation as well as the cross-sectional variation in the average excess stock returns of the 27 portfolios so that little is left unexplained in the intercepts. Formal GRS test cannot reject the null hypothesis that the intercepts are jointly insignificantly different from zero with an $F$ statistic equal to 1.31 and the $p$-value of 0.18 , which is a much stronger result than what is derived in the threefactor model. The average alpha is around 1.15 percent annually, which is an economically insignificant abnormal return. In short, the four-factor model seems to do a better job in explaining the 27 size-BE/ME-corporate liquidity portfolios than the threefactor model. 


\section{IV.5. Asset Pricing Models on 10 Corporate Liquidity Portfolios}

In order to further examine whether the corporate liquidity factor can explain the return differentials across firms with different cash holding levels, I sort the sample into 10 portfolios based on the firm's corporate liquidity, instead of size, book-to-market ratio and corporate liquidity. Then I run the CAPM, Fama-French three-factor model as well as the four-factor model similar to the previous section for the 10 corporate liquidity portfolios and look at the difference of the alphas as well as factor loadings.

The regression estimations are presented in Table 8. Panel A shows the results for the simple CAPM across the 10 corporate liquidity portfolios. The constants are insignificant for all the 10 groups. Furthermore, the market factor has a significant impact on these portfolios' excess returns. The loadings on the market factor show a substantial variation across the 10 corporate liquidity portfolios. Particularly, the excess equity returns of liquid firms are more sensitive to the market factor than illiquid portfolios. GRS test provides an $F$ value equal to 0.44 and a $p$-value of 0.93 , and we cannot reject the null hypothesis that the intercepts of the 10 portfolios are statistically insignificantly different from zero.

Panel B exhibits the regression results of the Fama-French three-factor model for the 10 corporate liquidity portfolios. The alphas are not significantly different from zero except for the most illiquid firms and the $6^{\text {th }}$ illiquid group. Market betas increase with the corporate liquidity. Size factor has the highest loading on the most liquid portfolio while the value factor has the highest loading on the most illiquid portfolio. GRS test fails to reject the null hypothesis.

Panel $\mathrm{C}$ shows the results of the augmented Fama-French three-factor model plus the corporate liquidity factor $L M I$. It is interesting to see the loadings on $L M I$ are negative for illiquid companies but positive for liquid companies. Furthermore, loadings on LMI increase along with the corporate liquidity, which is consistent with the regression results for the previous 27 size-value-corporate liquidity portfolios in the previous section. The four-factor regression on 10 corporate liquidity portfolios further confirms that there is a corporate liquidity factor that can explain the stock return variations across firms with different cash holding levels. The alphas are statistically insignificant across all the corporate liquidity portfolios, which indicates that the four factors have absorbed most of 
the return variations. F statistic from the GRS test is 0.56 with a $p$-value equal to 0.84 , which indicates that the null hypothesis cannot be rejected and the four-factor model provides a good fit to interpret the return variations across the 10 corporate liquidity portfolios.

\section{IV.6. Multifactor Asset Pricing Models on 25 Subsequently Sorted Size- or BE/ME-} Corporate Liquidity Portfolios

Finally, following Campbell, Hilscher and Szilagyi (2005), instead of independently sorting the firms into 27 size-BE/ME-corporate liquidity portfolios, I subsequently sort firms into 25 size-corporate liquidity and $25 \mathrm{BE} / \mathrm{ME}$-corporate liquidity portfolios, and run the Fama-French three-factor model for the two different double sorted 25 portfolios respectively. By comparing the three-factor model alphas across the 25 portfolios, we can see whether illiquid stocks underperform liquid stocks, and whether size and value effects exist significantly across liquidity portfolios. The results are summarized by Table 9 through 12 .

Table 9 shows the coefficients of three Fama-French factors for the 25 size-corporate liquidity sorted portfolios. Controlling for size, alphas are turning from negative to positive as firms become more liquid. On average, market betas are slightly higher for liquid firms than illiquid firms given their size. For each size group, the loadings on size factor increase steadily along with the cash holding; while the loadings on value factor turn from positive to negative. Within each size portfolio, the most liquid portfolio has the highest size loading but the most negative value loading. The $R$-squareds are satisfactory for all the 25 groups and suggest the three-factor model is good in interpreting the time series variation in stock returns for these portfolios. Table 10 presents the results for the $25 \mathrm{BE} / \mathrm{ME}$-corporate liquidity portfolios. The patterns of the loadings are similar to the 25 size-corporate liquidity portfolios.

Table 11 and 12 exhibit the four-factor time series regression result for the 25 sizecorporate liquidity and BE/ME-corporate liquidity portfolio respectively. The alphas now become statistically insignificant after the additional LMI factor is included in the regression, which indicates that the four-factor model does a better job in explaining the equity returns on average, and the $L M I$ factor absorbs the return variations that cannot be 
explained by the Fama-French three factors. However, it remains a puzzle that this is less obvious in the regressions for the 27 size-BE/ME-corporate liquidity portfolios. In addition, the loadings on the three factors $M K T, S M B$ and $H M L$ do not show any obvious pattern across the liquidity portfolios with the $L M I$ included the regression. Furthermore, similar to the three-factor model, loadings on $L M I$ change sign from negative to positive as corporate liquidity increases for all the size or BE/ME portfolios. Equity returns of liquid firms respond positively to the $L M I$ factor while illiquid firms respond in the opposite way. This clear pattern of $L M I$ loadings across corporate liquidity portfolios provides strong support for the existence of corporate liquidity factor. Finally, GRS tests for both models show that with a highly significant $F$ statistic, the jointly zero intercepts null hypothesis cannot rejected.

\section{Corporate Liquidity and Macroeconomic Variables}

We have found that there exists an equity premium for firms with higher corporate liquidity. The previous multifactor asset pricing models results show that the constructed $L M I$ factor actually captures the time series equity return variations across portfolios formed in different ways. Particularly, loadings on $L M I$ are higher for firms with higher corporate liquidity, and therefore we conclude that there is a corporate liquidity factor. Since the corporate liquidity factor is a risk factor, it is tempting to ask whether it is systematic, and what kind of risk it may proxy for.

\section{1. Is Corporate Liquidity Factor a Proxy for Systematic Risk?}

In order to further examine whether corporate liquidity is actually a risk factor, I explore the dynamics of corporate liquidity and whether it predicts changes in macroeconomic condition. According to Hypothesis 3, we should observe the LMI factor moving together with aggregate economic conditions over time if it can serve as a proxy for systematic risk. If corporate liquidity factor can proxy for the changes in future investment opportunity set, LMI should contain information to forecast future macroeconomic changes. Similar to Lamont, Polk and Saá-Requejo (2001) but with different 
macroeconomic variables, the time series regression of corporate liquidity on changes in macroeconomic condition has been estimated as follows:

$$
L M I_{t}=\alpha+\sum_{j=0}^{2} \delta_{j} \Delta \text { MacroCondition }{ }_{t+j}+\varepsilon_{t}
$$

$L M I$ is the aggregate corporate liquidity factor. Current and two future values of macroeconomic variables: $\triangle G D P, \triangle I P, \triangle C P I, \triangle F F R$ and NBER recession dummy are used to proxy for future macroeconomic condition changes that can affect operation performance, investment opportunities and financial condition.

Table 13 summarizes the regression results. It seems that the corporate liquidity factor is not very closely related to the changes in macroeconomics. The average return differential between firms with high and low cash holding level is not very sensitive to changes in economic condition or monetary policy.

\section{V.2. Are Liquid Firms' Fundamentals More Cyclical?}

Asset pricing literature suggests that possible sources of systematic risks come from the co-movement between equity returns and macroeconomic conditions. In the long run, equity returns are determined by business fundamentals. It is tempting to investigate whether and how the relationship between firms' performance and macroeconomic variables differentiates between liquid and illiquid firms. If Hypothesis 4 is true based on precautionary saving motive, we can expect that the fundamental business activities of more liquid firms are more cyclical than those of illiquid firms. If this is the case, then we can identify the corporate liquidity as a possible source of systematic risk in the sense that real performance is associated with economic conditions differently for liquid and illiquid firms. I follow Campello and Chen (2005) to examine the corporate liquidity and business fundamental sensitivities to changes in aggregate economy. The model to be estimated is as follows:

$$
\text { Performance }_{i, t}=\alpha+\delta \Delta \text { MacroCondition }_{t}+\phi \Delta F F R_{t}+\rho \text { Trend }_{t}+\varepsilon_{i, t}
$$

where Performance is measured by median growth rate of earnings before interest and taxes (EBIT, COMPUSTAT item 14 + item 18), sales (item 12) and investment (item 128). MacroCondition includes (1) aggregate demand, proxied by GDP-gross domestic production; (2) aggregate production, proxied by IP - industrial production index; (3) a 
dummy variable indicating recession period defined by NBER, and (4) federal funds rate $(F F R)$, a proxy for monetary policy. Federal funds rate is included in the bivariate regression with GDP to control for the monetary policy adjustments that can coincide with macroeconomic changes. In addition, the recession dummy variable takes a value of -1 for the year under recession in order to conveniently compare its regression coefficient with other macroeconomic condition changes. The above series can be obtained from the Bureau of Labor Statistics and from the Federal Reserve (Statistical Release H.15).

In every year all the firms are sorted according to their corporate liquidity position. Liquid firms are defined as those whose cash holdings fall into top 30 percentile, while illiquid firms are those with bottom 30 percentile cash holdings. The above time series regression is estimated for liquid and illiquid firms separately, and the coefficient on macroeconomic change variable $\boldsymbol{\delta}$ is compared for the two groups. If liquidity signals higher risks, we would expect that $\left|\delta_{\text {liquid }}\right|>\left|\delta_{\text {illiquid }}\right|$ since liquid firms are more cyclical.

Table 14 shows the regression results for both liquid portfolio and illiquid portfolio. In total there are 24 regressions: 3 performance variables, 4 macroeconomic change variable combinations for 2 liquidity portfolios. The regression results show that contrary to Hypothesis 4, illiquid firms are actually more cyclical compared to liquid firms. The coefficients on GDP, IP and the recession dummy variable are more pronounced for the illiquid group. When economic growth is strong, firms with a relatively lower level of financial slack have stronger sales growth, EBIT growth and investment growth; while illiquid companies tend to suffer more from a slower economy as their sales decline, profits slide and investment expenses are cut.

\section{V.3. Corporate Liquidity Beta and Changes in Macroeconomic Condition}

In Chapter 1, I test that whether corporate liquidity is positively correlated with firm beta. If we believe that beta is an appropriate measure for market risk, then liquid asset holdings signal a higher level of future risk for the firm if a liquid firm has higher beta. In this chapter, I study how the corporate liquidity beta responds to the macroeconomic risks, and whether the corporate liquidity beta behaves differently for liquid firms and illiquid firms. In order to examine this issue, I perform a two-step regression similar to Almeida, Campello and Weisbach (2004) and Campello (2003). In the first step, time series 
regressions are estimated for liquidity beta on a rolling basis for the liquid and illiquid portfolio separately every month using the previous 36 months data points, in which portfolios' excess returns are regressed on the factors $L M I, M K T, S M B$ and $H M L$. The coefficients on corporate liquidity factor $L M I$ are collected and "stacked" into the vector $\theta_{t}$, which is then regressed on changes in the various macroeconomic variables, including current and two lagged changes in GDP, and contemporaneous LogCPI, changes in the federal funds rate $\operatorname{LogFFR}$ and a time trend.

$$
\theta_{t}=\alpha+\sum_{k=0}^{2} \delta_{k} \Delta G D P_{t-k}+\varphi \operatorname{LogCPI}_{t}+\lambda \operatorname{LogFFR}_{t}+\rho \text { Trend }_{t}+\mu_{t}
$$

If higher levels of cash holdings are related to higher stock returns due to higher risks, then $\theta_{t}$ is expected to be higher when aggregate economic conditions become less favorable, hence the coefficient on $\triangle G D P$ is expected to have a negative sign. On the contrary, if higher cash holdings lead to higher stock returns because of good investment opportunities, $\theta_{t}$ should be higher when there is a positive aggregate demand shock, which indicates a positive coefficient on $\triangle G D P$.

The above two-step regression is performed on the portfolios containing firms with top 30 percentile and bottom 30 percentile cash holdings to compare the impact of macrochanges on corporate liquidity beta between high and low corporate liquidity firms. If firms holding excessively low levels of cash are subject to more default risk, these firms should have positive and higher coefficients on macroeconomic factors compared to firms with higher cash holdings.

A summary for the two-step regression results is presented in Table 15. In general, coefficients on GDP changes are negative for both liquid and illiquid portfolios. When macroeconomic condition becomes less favorable, this increases the corporate liquidity beta for firms with both high and low cash holdings. Furthermore, it seems that the corporate liquidity beta is more sensitive to the macroeconomic changes for liquid firms than illiquid firms. This observation strengthens the idea that companies pile up cash for precautionary purposes. If there is an economic downtown, those firms which are more sensitive to the less favorable economic conditions will increase the liquid asset percentage in total assets. Higher sensitivity to the macroeconomic changes indicates higher risks. Therefore, based on the two-step regression, firms with higher corporate 
liquidity are riskier than less liquid firms. As a robustness check, rolling corporate liquidity betas are estimated using the previous 60 months' data points in the first pass, and the empirical results in the second step remain similar.

\section{V.4. Do Financial Constraints Affect the Relationship Between Corporate Liquidity Betas and Changes in Macroeconomic Variables?}

The last issue to be addressed is whether the corporate liquidity beta of financially constrained firms behaves differently from that of financially unconstrained firms when macroeconomic condition changes. Almeida, Campello and Weisbach (2004) present a model in which companies respond to potential financial constraints by hoarding more cash today. Furthermore, Lamont, Polk and Saá-Requejo (2001) find that stock returns of financially constrained firms tend to move together, which indicates that these firms are subject to common shocks. Campello and Chen (2005), Whited and Wu (2006) show that constrained firms earn higher returns and external finance constraints do indeed represent a risk factor that is priced in financial markets. A reasonable extension of the above literature would be that if financially constrained firms are riskier, which is the reason for these firms to maintain a high level of liquidity, such firms will have higher expected stock returns. The macroeconomic sensitivity of corporate liquidity should be more pronounced for companies that are financially constrained.

The sample is split into constrained and unconstrained firms based on the following criteria: firm size, payout ratio and a $\mathrm{KZ}$ index. Then the previous two-step combination of cross sectional and time series regressions is performed for the constrained group and unconstrained group separately. If cash holding is more valuable for constrained firms, then a positive economic shock increases the benefit of keeping cash for constrained firms and reduces the risk; on the other hand, an adverse macroeconomic condition can impose more hardship for financially constrained companies, whose corporate liquidity beta will be more responding to the negative shock. Therefore, it is reasonable to expect that the coefficients on $\triangle G D P$ are negative and more remarkable for constrained firms, if financial constraint is considered as a risk.

Table 16 presents the results for constrained and unconstrained portfolios defined by three different criteria. A negative shock increases the corporate liquidity beta for both 
the constrained and unconstrained firms. As we expected, the corporate liquidity beta of the constrained group responds to the GDP growth more pronouncedly compared to unconstrained group. For firms with difficulty in accessing external financial markets, their corporate liquidity beta is more sensitive to fluctuations in macroeconomic conditions and is greater in absolute value than firms who are not financially constrained. The empirical results provides evidence to support the view that firms hoard cash and liquid assets because they are financially constrained and therefore more exposed to macroeconomic risks.

\section{Conclusion}

This paper investigates whether aggregate corporate liquidity can be a systematic risk factor that is priced in an asset pricing model. Asset-pricing tests are performed to examine whether the corporate liquidity factor is a risk factor and whether it is systematic and priced. Empirical results have confirmed that although the three popular factors $M K T$, $S M B$ and $H M L$ share some information contained in the corporate liquidity factor, $L M I$ can still serve as an independent factor and is priced.

By comparing the results from Fama-French three-factor model and the four-factor model on 27 portfolios formed by independently sorting stocks by size, book-to-market equity and corporate liquidity, it can be shown that the loadings of the SMB and $H M L$ factors change when the additional liquidity factor is included in the time-series regression. In particular, the explanatory power of $S M B$ strengthens while the loadings of $H M L$ weaken substantially for the most liquid portfolios.

The empirical results show that $L M I$ certainly captures the time-series variation of the excess stock returns. The loadings of $L M I$ turn negative to positive from most illiquid portfolios to the most liquid portfolios, with all the slopes for the liquid portfolios highly significant. The different signs of $L M I$ loadings indicate that the corporate liquidity factor loads on illiquid firms and liquid firms in an opposite way. LMI lowers the average expected returns on illiquid firms while increasing the average returns on more liquid firms. The Gibbons-Ross-Shanken test cannot reject the null hypothesis that the timeseries regression intercepts are jointly insignificantly different from zero and the four- 
factor model actually well explains the common variations in a cross-section of excess stock returns.

In addition, CAPM, Fama-French three-factor model and the four-factor model are estimated for the 10 portfolios constructed based on the corporate liquidity. The results show that liquid portfolios have higher market betas than illiquid ones, and loadings on LMI increase along with the corporate liquidity. Compared to CAPM and Fama-French three-factor model, the four-factor regression better explains the stock return variations across firms with different cash holding levels.

Furthermore, multifactor regressions are performed for the 25 subsequently sorted size - corporate liquidity and BE/ME-corporate liquidity portfolios. The empirical results strongly suggest the existence of corporate liquidity effect.

More empirical estimations are completed to examine whether the corporate liquidity factor is systematic and what kind of risk it may proxy for. I have not found clear evidence that the $L M I$ factor co-moves with the macroeconomic variables. It seems that he average return differential between firms with high and low level of cash holding is not sensitive to changes in economic condition or monetary policy. Furthermore, when looking at the relationship between companies' performance and macroeconomic conditions, the regression results show illiquid firms are actually more cyclical than liquid firms. The coefficients on GDP, IP and the Recession dummy variable are more pronounced for the illiquid group. When economic growth gets stronger, illiquid firms tend to experience stronger sales growth, EBIT growth and investment growth; while these illiquid companies tend to suffer more from a slower economy as their sales decline, profits slide and investment expenses are cut.

Moreover, I also find that negative changes in macroeconomic conditions will increase the corporate liquidity beta, and it seems that the corporate liquidity beta is more sensitive to the macroeconomic fluctuations for liquid firms than illiquid firms. These results support the idea that companies hoard cash and cash equivalents for precautionary purposes. If there is an economic recession, those firms who are more sensitive to the less favorable economic condition will increase the liquid asset holding. Firms with higher corporate liquidity are riskier than less liquid firms because their higher sensitivity to the macroeconomic changes signals higher risks. Finally, I examine whether the corporate 
liquidity beta of financially constrained firms and unconstrained firms responds differently to a change in macroeconomic conditions. There is some evidence showing that the corporate liquidity beta of financially constrained companies is more sensitive to the macroeconomic changes and is greater in absolute value than for firms who are not financially constrained. The empirical results offer evidence to support the view that firms increase asset liquidity because they are financially constrained and therefore more exposed to macroeconomic risks. 


\section{APPENDIX of Chapter 2}

This appendix examines the multifactor models with decomposed corporate liquidity factors. In addition, I also investigate whether corporate liquidity can forecast future equity returns.

In Part 4 of Section IV, we have presented a four-factor asset pricing models on 27 Size-BE/ME-Corporate liquidity portfolios. From the four-factor model it can be shown that expected stock returns are sensitive to the liquidity factor. Specifically, illiquid firms load negatively while liquid firms load positively on the $L M I$ factor. In order to further explore whether the effect the corporate liquidity factor on expected equity returns varies along the corporate liquidity spectrum, I split the overall $L M I$ factor into two factors to capture the risks associated with liquidity constraint and excess liquidity. These two sub liquidity factors are constructed in order to examine whether the importance of corporate liquidity factor varies with the degree of corporate liquidity itself. In another word, we would like to find out whether corporate liquidity effect is stronger for more high cash holding firms or low cash holding firms.

\section{A.1. The Four-Factor Model with Decomposed Corporate Liquidity Factors}

The overall corporate liquidity factor is decomposed into 2 sub-liquidity factors $L M I_{\text {med_low } t}$ and $L M I_{\text {high } \text { med }}$, where $L M I_{\text {med_low } t}$ stands for "Medium Minus Low" and $L M I_{\text {high_med } t}$ stands for "High Minus Medium". $L M I_{\text {med_low }}$ is a zero-investment portfolio that is long stocks with medium level liquid asset and short stocks with the least liquid asset holdings. It is constructed by taking the difference between the average returns of the medium 40 percentile liquidity level stocks and the bottom 30 percentile illiquid stocks. LMI high_med $t$ is a zero-investment portfolio that is long stocks with the highest corporate liquidity and short stocks with the medium liquid asset holdings. It is constructed by taking the return differential between the top 30 percentile liquid stocks and the medium 40 percentile liquidity stocks. $L M I_{\text {med_low } t}$ aims to capture the effect of liquidity constraints imposed on the expected equity returns while LMI high med $t$ is designed to examine the impact of excess liquidity on the expected equity returns. 
The four-factor models with the 2 decomposed liquidity factors respectively estimated in this section are as follows:

$$
\begin{gathered}
R_{t}^{i}-R F_{t}=a^{i}+b^{i} M K T_{t}+s^{i} S M B_{t}+h^{i} H M L_{t}+c^{i} L M I_{\text {med_low }_{-}}+e_{t} \\
R_{t}^{i}-R F_{t}=a^{i}+b^{i} M K T_{t}+s^{i} S M B_{t}+h^{i} H M L_{t}+c^{i} L M I_{\text {high_med }_{t}}+e_{t},
\end{gathered}
$$

Table A1 and Table A2 exhibit the results of the four-factor model with corporate liquidity factor separated into two parts. The loadings on both the liquidity constraint factor and the excess liquidity factor behave quite differently across liquidity portfolios, which is consistent with the four-factor model using overall corporate liquidity factor as the fourth factor. It can be shown in Table A1 that the loadings on LMI med_low $t$ are negative and highly significant for the most illiquid portfolios, while insignificant for medium liquid and liquid portfolios. This indicates that firms with low level of liquid assets holdings are more sensitive to the liquidity constraint factor while firms with higher corporate liquidity do not. For firms with lower level of liquid assets, holding less cash will increase the required returns of their equity substantially, because of the higher probability of financial distress. GRS test fails to reject the null hypothesis that the intercepts of the 27 portfolios are jointly insignificantly different from zero.

Table A2 shows that the slopes on $L M I_{\text {high_med }}$ are strongly significant and positive for almost all the liquid portfolios, which is consistent with the four-factor regression with overall corporate liquidity factor $L M I$. For those firms that hold a high proportion of cash, increasing corporate liquidity will raise the required returns as investors may believe that those firms may keep a high level of financial slack for the precautionary purpose because of volatile cash flows or underlying operational risks, which sends a signal to the market that these firms are really risky. If investors realize this they will require a higher rate of return in order to hold these firms' equity. An alternative interpretation would be that current stock prices are pushed down because investors think liquid firms lack investment opportunities and hoard cash inefficiently, leading to high required returns. Most of the slopes of the excess liquidity factor on the illiquid and medium liquidity portfolios are, however, not significant and have mixed signs. Firms keeping a low or moderate proportion of cash are not sensitive to the excess liquidity factor. F statistic of 
the GRS test is 1.43 with a $p$-value equal to 0.09 , so that the null hypothesis can be rejected at the 10 percent significance level while cannot be rejected at the 5 percent level.

\section{A.2. The Five-Factor Model with Decomposed Corporate Liquidity Factors}

Next, a five-factor model is estimated to incorporate both liquidity constraint factor and excess liquidity factor.

$$
R_{t}^{i}-R F_{t}=a^{i}+b^{i} M K T_{t}+s^{i} S M B_{t}+h^{i} H M L_{t}+c_{1}^{i} L M I \text { med_low }{ }_{t}+c_{2}^{i} L M I_{\text {high }_{-} \text {med }_{t}}+e_{t}
$$

Table A3 shows that the loadings on $L M I_{\text {med_low }_{t}}$ and $L M I_{\text {high } \text { med }_{t} \text { are quite consistent }}$ with those estimated in the separate four-factor models. Particularly, the slopes of the factor LMI med_low $t$ are significantly negative for the most illiquid portfolios while insignificant for medium and liquid portfolios. Furthermore, $L M I_{\text {high_med }}$ loads positively and significantly for the most liquid portfolios while insignificantly for these illiquid and medium liquid portfolios. Again, the five-factor model confirms that illiquid firms are sensitive to the liquidity constraint factor, and lower corporate liquidity is associated with higher expected equity returns; while liquid firms are sensitive to the excess liquidity factor, and higher corporate liquidity leads to higher expected returns for these stocks. GRS test result is similar to the four factor model with $L M I_{\text {high } \_ \text {med }}$, and the intercepts of the 27 portfolios are significantly different from zero at a 10 percent level while insignificantly different from zero at a 5 percent level.

To summarize, it seems that the impact of corporate liquidity factor on cross-sectional

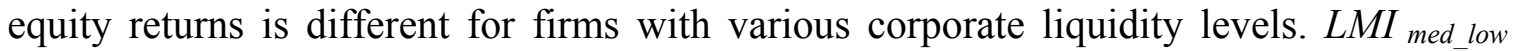
better explains return variations for firms falling into the medium-to-low corporate liquidity cohort, while $L M I_{\text {high_med }}$ better interprets return variations for firms with the high-to-medium liquidity level. Furthermore, according to the GRS test, the explanatory power of the multifactor model with the $L M I_{\text {med_low }}$ factor is stronger compared to the model with $L M I_{\text {high_med }}$ factor.

\section{B. Future Equity Return Forecast Using Corporate Liquidity}

Greenwood (2004) studies the relationship between investment in cash and liquid assets as a share of total investment, and subsequent market returns. He finds that changes in aggregate cash have significant incremental predictive power. In aggregate 
level, increases in cash frequently predict negative equity returns while these changes are not correlated with planned investment expenditures and cannot be explained by current or lagged changes in profitability. Greenwood concludes that the empirical evidence supports the active market timing theory, in which cash accumulation is a consequence of additional equity issued by overvalued firms who cannot spend funds productively and do not return cash to investors.

Greenwood (2004) uses the data from Federal Reserve Flow of Funds to construct a measure of aggregate corporate investment in cash and financial securities; and uses OLS regressions to study the predictive power of aggregate cash. In stead, I adopt the portfolio construction approach to examine this issue.

In order to investigate whether corporate liquidity can forecast future equity returns, I construct 9 zero-investment size-BE/ME portfolios that are long liquid stocks and short illiquid stocks and calculate the returns for a subsequent 6-month holding period. Specifically, in each June and December, the entire sample is sorted into 9 size-BE/ME portfolios. Within each portfolio, all the stocks are further sorted into 2 portfolios: liquid portfolio, which includes firms whose corporate liquidity is ranked in the top 30 percentile, and illiquid portfolio, which includes firms whose corporate liquidity is ranked in the bottom 30 percentile. The 9 zero-investment portfolios are constructed as long the relatively liquid firms and short the relatively illiquid firms. Cumulative portfolio returns are calculated as the equal-weighted average of cumulative individual stock returns. Finally, geometric averages of cumulative portfolio returns for the 6 months following the month when the portfolios are formed (excluding the immediate subsequent month) are calculated. To separate the effect of liquidity constraint and excess liquidity, the 9 zero-investment size-BE/ME portfolios are alternatively formed as long the medium level liquidity firms (40-70 percentiles) and short the illiquid firms, and long the liquid firms and short the medium level liquidity firms.

Table B displays the results of the forecast ability of corporate liquidity. Panel A shows that the trading strategy that longs top 30 percentile liquid firms and shorts bottom 30 percentile illiquid firms brings a positive monthly return of about 1.45 percent for small-growth firms. This is not only statistically but also economically significant. Small firms and growth firms earn positive returns but the returns are not significant. Panel B 
shows that if investors hold the zero-investment portfolios that long medium liquid firms and short illiquid firms for 6 months, they can earn significantly positive returns on average for small-growth portfolios and small-value portfolios. All the 9 portfolios bring positive monthly returns if they are held for 6 months, though most are not significant. Panel $\mathrm{C}$ displays the average monthly returns for the 9 portfolios that long liquid firms and short medium liquid firms. Medium size-growth firms and large-growth firms have negative monthly returns, implying that firms with high levels of liquid assets underperform firms with medium levels of liquid assets during a 6-month subsequent period. We can conclude that medium liquid firms do very well on average. 


\section{REFERNCES}

Acharya, Viral, and Lasse H. Pedersen, 2005, Asset Pricing with Liquidity Risk, Journal of Financial Economics 77, 375-410.

Almeida, Heitor, Murillo Campello, and Michael S. Weisbach, 2002, Corporate Demand for Liquidity, NBER working paper No. W9253.

Almeida, Heitor, Murillo Campello, and Michael S. Weisbach, 2004, The Cash Flow Sensitivity of Cash, Journal of Finance 59, 1777-1804.

Banz, Rolf W., 1981, The Relationship Between Return and Market Value of Common Stocks, Journal of Financial Economics 9, 3-18.

Baskin, Jonathan B., 1987, Corporate Liquidity in Games of Monopoly Power, Review of Economics and Statistics 69, 312-319.

Basu, Sanjoy, 1977, Investment Performance of Common Stocks in Relation to Their Price-Earnings Ratios: A Test of the Efficient Market Hypothesis, Journal of Finance $32,663-682$.

Basu, Sanjoy, 1983, The Relationship Between Earnings' Yield, Market Value and Return For NYSE Common Stocks, Journal of Financial Economics 9, 129-156.

Bhandari, Laxmi Chand, 1988, Debt/Equity Ratio and Expected Common Stock Returns: Empirical Evidence, Journal of Finance 43, 507-528.

Brennan, Michael J., and Avanidhar Subrahmanyam, 1996, Market Microstructure and Asset Pricing: On the Compensation for Illiquidity in Stock Returns, Journal of Financial Economics 41, 441-464.

Campbell, John Y., Jens Hilscher, and Jan Szilagyi, 2005, In Search of Distress Risk, Harvard Institute of Economic Research Discussion Paper No. 2081, Harvard University.

Campello, Murillo, 2003, Capital Structure and Product Markets Interactions: Evidence from Business Cycles, Journal of Financial Economics 68: 353-378.

Campello, Murillo, and Long Chen, 2005, Are Financial Constraints Priced? Evidence from Firm Fundamentals, Stocks, and Bonds, AFA 2006 Boston Meeting.

Chordia, Tarun, Richard Roll, and Avanidhar Subrahmanyam, 2000, Commonality in Liquidity, Journal of Financial Economics 56, 3-28.

Cohen, Randolph B., Christopher Polk, and Tuomo Vuolteenaho, 2003, The Value Spread, Journal of Finance 58, 609-641. 
Constantinides, George M., 1989, Theory of Valuation: Overview and Recent Developments, in S. Bhattacharya and George M. Constantinides (eds.) Theory of Valuation: Frontiers of Modern Financial Theory, Volume 1, Totowa, N.J. Rowman and Littlefield.

Damodaran, Aswath, 2005, Dealing with Cash, Cross Holdings and Other Non-Operating Asset: Approaches and Implications, working paper.

Easley, David, Soeren Hvidkjaer, and Maureen O'Hara, 2002, Is Information a determinant of asset returns? Journal of Finance 57, 2185-2221.

Easley, David, Nicholas. M. Kiefer, and Maureen O'Hara, 1997, One Day in the life of a very Common Stock, Review of Financial Studies 10, 805-835.

Easley, David, and Maureen O'Hara, 2003, Microstructure and Asset Pricing, Handbook of the Economics of Finance, Edited by G.M. Constantinides, M. Harris, and R. Stulz.

Easley, David, and Maureen O'Hara, 2004, Information and the Cost of Capital, Journal of Finance 59, 1553-1583.

Fama, Eugene F., and Kenneth R. French, 1992, The Cross-section of Expected Stock Returns. Journal of Finance 47, 427-465.

Fama, Eugene F., and Kenneth R. French, 1993, Common Risk Factors in the Returns on Stocks and Bonds. Journal of Financial Economics 33, 3-56.

Fama, Eugene F., and Kenneth R. French, 1995, Size and Book-to-Market Factors in Earnings and Returns, Journal of Finance 50, 131-155.

Fama, Eugene F., and Kenneth R. French, 1996, Multifactor Explanations of Asset Pricing Anomalies, Journal of Finance 51, 55-84.

Fama, Eugene F., and James MacBeth, 1973, Risk, Return and Equilibrium: Empirical Tests, Journal of Political Economy 81, 607-636.

Faulkender, Michael, and Rong Wang, 2006, Corporate Financial Policy and the Value of Cash, Journal of Finance 61, 1957-1990.

Gibbons, Michael R., Stephen Ross and Jay Shanken, 1989, A Test of the Efficiency of a Given Portfolio, Econometrica 57, 1121-1152.

Greenwood, Robin, 2004, Aggregate Corporate Liquidity and Stock Returns, Harvard Business School Working Paper, No. 05-014 
Grossman, Sanford J., and Joseph E. Stiglitz, 1980, On the Impossibility of Informationally Efficient Markets, American Economic Review 70, 393-408.

Holmström, Bengt, and Jean Tirole, 2001, LAPM: A Liquidity-Based Asset Pricing Model, Journal of Finance 56, 1837-1867.

Kaplan, Steven N., and Luigi Zingales, 1997, Do Investment-Cash Flow Sensitivities Provide Useful Measures of Financing Constraints? Quarterly Journal of Economics $112,169-215$.

Lamont, Owen, Christopher Polk and Jesús Saá-Requejo, 2001, Financial Constraints and Stock Returns, The Review of Financial Studies 14, 529-554.

Liew, Jimmy, and Maria Vassalou, 2000, Can Book-to-Market, Size, and Momentum be Risk Factors that Predict Economic Growth? Journal of Financial Economics 57, 221-245.

Merton, Robert C., 1973, An Intertemporal Capital Asset Pricing Model, Econometrica $41,867-887$.

Merton, Robert C., 1990, Continuous-Time Finance. Oxford, U.K.: Basil Blackwell.

Mikkelson, Wayne H., and M. Megan Partch, 2003, Do Persistent Large Cash Reserve Hinder Performance? Journal of Financial and Quantitative Analysis 38, 275-294.

Opler, Tim, Lee Pinkowitz, Rene Stulz, and Rohan Williamson, 1999, The Determinants and Implications of Corporate Cash Holdings, Journal of Financial Economics 52, 346.

Pástor, Luboš, and Stambaugh, Robert F., 2003, Liquidity Risk and Expected Stock Returns, Journal of Political Economy 111, 642-685.

Pinkowitz, Lee, and Rohan Williamson, 2004, What is a Dollar Worth? The Market Value of Cash Holdings, working paper, Georgetown University.

Ross, Stephen A., 1976a, The Arbitrage Theory of Capital Asset Pricing, Journal of Economic Theory 13, 341-360.

Ross, Stephen A., 1976b, Risk, Return, and Arbitrage, In Risk and Return in Finance, Edited by Irwin Friend and James Bicksler, 189-218. Cambridge, MA.: Ballinger.

Sibilkov, Valeriy, 2005, Is Cash More Valuable for Constrained Firms? working paper, Purdue University.

Stoll, Hans R., and Robert E. Whaley, 1983, Transaction Costs and the Small Firm Effect, Journal of Financial Economics 12, 57-79. 
Vassalou, Maria, and Yuhang Xing, 2004, Default Risk in Equity Returns, Journal of Finance 59, 831-868. 


\section{Table 1. Summary Statistics for the 1962.1 - 2005.12 Sample}

Stocks are sorted into 10 portfolios on the basis of return, size, BE/ME, corporate liquidity (CL), leverage, CL deviation and Leverage deviation. Sample mean and standard deviation, and average of each quintile are displayed in Panel A. SIZE denotes the market value of the stocks, measured in June of each year. BE/ME denotes the book-to-market equity. CL is the corporate liquidity, measured as the ratio of cash and marketable securities to total assets. Leverage is debt-to-asset ratio. In order to remove the effect of industry differentials, CL deviation and Leverage deviation denote the deviation of firms' cash holdings and financial leverage compared to the industry average based on the Fama-French 17 industries. Panel B shows the mean and t statistics for the factors. $M K T, S M B$ and $H M L$ are Fama-French three factors. $L M I$ denotes "Liquid minus illiquid", which is a factor constructed by taking the difference between the average returns of the top 30 percent liquid stocks and the bottom 30 percent illiquid stocks. $L M I_{m e d}$ low denotes "medium minus low", which is a factor constructed by taking the difference between the average returns of the medium 40 percentile liquidity level stocks and the bottom 30 percentile illiquid stocks. $L M I_{\text {high med }}$ denotes "high minus medium", which is a factor constructed by taking the difference between the average returns of the top 30 percentile liquid stocks and the medium 40 percentile liquidity level stocks. Panel $\mathrm{C}$ presents the Pearson correlation matrix of the factors, $p$-values are in parenthesis.

\begin{tabular}{|c|c|c|c|c|c|c|c|c|c|c|c|c|}
\hline Variable & Mean & $1 \mathrm{st}$ & $2 \mathrm{nd}$ & $3 \mathrm{rd}$ & 4 th & 5 th & 6 th & 7 th & 8 th & 9 th & 10th & $\begin{array}{l}\text { Std. } \\
\text { Dev. }\end{array}$ \\
\hline Mret & 1.432 & -27.465 & -13.794 & -8.153 & -4.353 & -1.180 & 1.389 & 4.729 & 8.940 & 15.672 & 38.537 & 18.223 \\
\hline$S I Z E$ & 1123.1 & 5.2 & 13.9 & 26.9 & 47.2 & 81.1 & 144.2 & 268.3 & 535.6 & 1279.4 & 8829.1 & 3914.2 \\
\hline$B E / M E$ & 1.835 & 0.081 & 0.186 & 0.279 & 0.374 & 0.482 & 0.613 & 0.779 & 1.012 & 1.451 & 13.091 & 8.596 \\
\hline$C L$ & 0.183 & 0.004 & 0.012 & 0.024 & 0.042 & 0.071 & 0.113 & 0.173 & 0.266 & 0.415 & 0.711 & 0.220 \\
\hline CL deviation & 0.000 & -0.245 & -0.170 & -0.122 & -0.090 & -0.068 & -0.041 & 0.006 & 0.079 & 0.202 & 0.446 & 0.195 \\
\hline $\begin{array}{l}\text { Leverage } \\
\text { Deviation }\end{array}$ & 0.000 & -0.205 & -0.162 & -0.145 & -0.116 & -0.074 & -0.022 & 0.034 & 0.100 & 0.188 & 0.401 & 0.185 \\
\hline
\end{tabular}


Table 1. (continued)

Panel B.

\begin{tabular}{lrr} 
Variable & Mean & t-stat \\
\hline$M K T$ & 0.448 & 4.448 \\
$S M B$ & 0.229 & 3.206 \\
$H M L$ & 0.464 & 2.896 \\
$L M I$ & 0.753 & 3.365 \\
LMI $_{\text {med_low }}$ & 0.285 & 1.307 \\
LMI $_{\text {high med }}$ & 0.538 & 2.723 \\
\hline
\end{tabular}

Panel C.

\begin{tabular}{|c|c|c|c|c|c|c|}
\hline & $M K T$ & $S M B$ & $H M L$ & $L M I$ & $L M I_{\text {med low }}$ & LMI $I_{\text {high med }}$ \\
\hline$M K T$ & $\begin{array}{c}1 \\
(0.000)\end{array}$ & $\begin{array}{c}0.302 \\
(0.000)\end{array}$ & $\begin{array}{c}-0.410 \\
(0.000)\end{array}$ & $\begin{array}{c}0.266 \\
(0.000)\end{array}$ & $\begin{array}{c}0.242 \\
(0.000)\end{array}$ & $\begin{array}{c}0.252 \\
(0.000)\end{array}$ \\
\hline$S M B$ & & $\begin{array}{c}1 \\
(0.000)\end{array}$ & $\begin{array}{l}-0.277 \\
(0.000)\end{array}$ & $\begin{array}{c}0.348 \\
(0.000)\end{array}$ & $\begin{array}{c}0.249 \\
(0.000)\end{array}$ & $\begin{array}{c}0.374 \\
(0.000)\end{array}$ \\
\hline$H M L$ & & & $\begin{array}{c}1 \\
(0.000)\end{array}$ & $\begin{array}{l}-0.629 \\
(0.506)\end{array}$ & $\begin{array}{l}-0.537 \\
(0.000)\end{array}$ & $\begin{array}{c}-0.613 \\
(0.000)\end{array}$ \\
\hline$L M I$ & & & & $\begin{array}{c}1 \\
(0.000)\end{array}$ & $\begin{array}{c}0.875 \\
(0.000)\end{array}$ & $\begin{array}{c}0.951 \\
(0.000)\end{array}$ \\
\hline$L M I_{m e d \_l o w}$ & & & & & $\begin{array}{c}1 \\
(0.000)\end{array}$ & $\begin{array}{c}0.689 \\
(0.000)\end{array}$ \\
\hline$L M I_{\text {high_med }}$ & & & & & & $\begin{array}{c}1 \\
(0.000)\end{array}$ \\
\hline
\end{tabular}

\section{Table 2. Pricing Tests on the Corporate Liquidity Factor}

This table reports results from regressions of corporate liquidity factors (LMI, LMI med_low, LMI high_med $)$ on Fama-French 3 risk factors $(M K T$, SMB and HML). $t$-statistics are reported in parentheses. The sample period is from January 1962 to December 2005, 528 months. $t$-statistics are in parenthesis.

\begin{tabular}{|c|c|c|c|c|}
\hline & (1) & (2) & (3) & (4) \\
\hline & $L M I$ & $L M I$ & $L M I_{\text {med low }}$ & $L M I_{\text {high med }}$ \\
\hline \multirow[t]{2}{*}{ Intercept } & 0.663 & 0.636 & 0.381 & 0.756 \\
\hline & $(4.668)^{* * *}$ & $(9.047)^{* * *}$ & $(7.720)^{* * *}$ & $(8.118)^{* * *}$ \\
\hline \multirow[t]{2}{*}{$M K T$} & 0.201 & 0.126 & 0.131 & 0.142 \\
\hline & $(6.335)^{* * *}$ & $(1.936)^{*}$ & $(1.842)^{*}$ & $(1.998)^{* *}$ \\
\hline \multirow[t]{2}{*}{$S M B$} & - & 0.205 & 0.044 & 0.197 \\
\hline & - & $(5.545)^{* * *}$ & $(2.770)^{* * *}$ & $(6.559)^{* * *}$ \\
\hline \multirow[t]{2}{*}{$H M L$} & - & -0.685 & -0.229 & -0.537 \\
\hline & - & $(-16.049)^{* * *}$ & $(-12.437)^{* * *}$ & $(-15.449)^{* * *}$ \\
\hline Adjusted $R^{2}$ & 0.071 & 0.430 & 0.299 & 0.424 \\
\hline
\end{tabular}


Table 3a. The Fama-French Three-Factor Model on 25 Size-BE/ME Portfolios

Time series regressions of excess stock returns on the excess market returns $(M K T)$ and the returns on size $(S M B)$ and book-to-market equity (HML) factors: July 1963 to December 2005, 510 months.

$$
R_{t}^{i}-R F_{t}=a^{i}+b^{i} M K T_{t}+s^{i} S M B_{t}+h^{i} H M L_{t}+e_{t}
$$

Dependent variable: Excess returns on 25 stock portfolios formed on size and book-to-market equity

Size quintiles

\begin{tabular}{rrrrr} 
Low & 2 & 3 & 4 & High \\
\hline \multicolumn{5}{c}{$\mathrm{a}$} \\
\hline 0.30 & -0.22 & -0.06 & 0.28 & 0.72 \\
0.39 & 0.01 & 0.12 & 0.32 & 0.33 \\
0.32 & 0.13 & 0.21 & 0.23 & 0.22 \\
0.38 & -0.09 & 0.15 & 0.20 & 0.31 \\
0.65 & 0.10 & 0.13 & 0.14 & 0.29 \\
\hline
\end{tabular}

$\mathrm{b}$

\begin{tabular}{rrrrrr} 
Small & 1.16 & 1.29 & 1.22 & 1.09 & 0.98 \\
2 & 1.15 & 1.15 & 1.13 & 1.10 & 1.03 \\
3 & 0.91 & 1.05 & 1.08 & 1.07 & 1.06 \\
4 & 0.93 & 1.04 & 1.08 & 1.09 & 1.10 \\
Big & 0.97 & 0.96 & 1.08 & 1.10 & 1.12 \\
\hline
\end{tabular}

\begin{tabular}{rrrrrr} 
& \multicolumn{5}{c}{$\mathrm{s}$} \\
\cline { 2 - 6 } Small & 1.78 & 1.31 & 1.05 & 0.74 & 0.21 \\
2 & 1.26 & 1.12 & 0.94 & 0.68 & 0.07 \\
3 & 1.21 & 1.01 & 0.75 & 0.55 & 0.21 \\
4 & 1.30 & 0.95 & 0.82 & 0.56 & 0.13 \\
Big & 1.22 & 0.95 & 0.88 & 0.60 & 0.20 \\
\hline
\end{tabular}

\begin{tabular}{rrrrrr} 
& \multicolumn{7}{c}{$\mathrm{h}$} \\
\cline { 2 - 6 } Small & 1.13 & 0.62 & 0.51 & -0.03 & -0.03 \\
2 & 0.59 & 0.41 & 0.39 & 0.29 & 0.28 \\
3 & 0.67 & 0.48 & 0.47 & 0.46 & 0.48 \\
4 & 0.82 & 0.75 & 0.74 & 0.59 & 0.53 \\
Big & 0.82 & 0.70 & 0.78 & 0.76 & 0.59 \\
\hline
\end{tabular}

\begin{tabular}{rlllll} 
& \multicolumn{5}{c}{$\mathrm{R}^{2}$} \\
\cline { 2 - 6 } Small & 0.77 & 0.87 & 0.84 & 0.81 & 0.84 \\
2 & 0.81 & 0.84 & 0.79 & 0.74 & 0.78 \\
3 & 0.82 & 0.81 & 0.73 & 0.72 & 0.71 \\
4 & 0.83 & 0.81 & 0.68 & 0.69 & 0.72 \\
Big & 0.85 & 0.74 & 0.72 & 0.68 & 0.73 \\
\hline
\end{tabular}

GRS test: $F$ value $=2.95$, $p$-value $=0.00$

\begin{tabular}{|c|c|c|c|c|}
\hline Low & 2 & 3 & 4 & High \\
\hline \multicolumn{5}{|c|}{$\mathrm{t}(\mathrm{a})$} \\
\hline 0.81 & -0.73 & -0.24 & 1.02 & 3.67 \\
\hline 1.30 & 0.01 & 0.44 & 1.49 & 1.57 \\
\hline 1.12 & 0.55 & 0.85 & 0.85 & 0.97 \\
\hline 1.49 & -0.33 & 0.47 & 0.74 & 1.13 \\
\hline 3.06 & 0.27 & 0.42 & 0.37 & 0.92 \\
\hline \multicolumn{5}{|c|}{$\mathrm{t}(\mathrm{b})$} \\
\hline 9.10 & 14.60 & 15.86 & 21.30 & 25.53 \\
\hline 11.81 & 17.77 & 17.75 & 27.38 & 33.42 \\
\hline 11.20 & 17.98 & 28.64 & 28.07 & 32.99 \\
\hline 13.58 & 23.07 & 29.90 & 32.14 & 30.25 \\
\hline 19.35 & 22.68 & 28.49 & 25.67 & 28.57 \\
\hline \multicolumn{5}{|c|}{$\mathrm{t}(\mathrm{s})$} \\
\hline 10.60 & 11.34 & 10.44 & 10.99 & 4.06 \\
\hline 9.80 & 13.16 & 11.23 & 12.84 & 1.68 \\
\hline 11.40 & 13.07 & 15.04 & 10.89 & 4.97 \\
\hline 14.32 & 16.03 & 17.26 & 12.55 & 2.72 \\
\hline 18.52 & 17.06 & 17.53 & 10.53 & 3.96 \\
\hline \multicolumn{5}{|c|}{$\mathrm{t}(\mathrm{h})$} \\
\hline 5.82 & 4.64 & 4.37 & -0.37 & -0.54 \\
\hline 3.97 & 4.18 & 4.04 & 4.69 & 5.85 \\
\hline 5.40 & 5.37 & 8.24 & 7.89 & 9.87 \\
\hline 7.85 & 10.88 & 13.46 & 11.41 & 9.63 \\
\hline 10.81 & 10.90 & 13.55 & 11.68 & 9.94 \\
\hline \multicolumn{5}{|c|}{$s(e)$} \\
\hline 11.39 & 8.21 & 6.65 & 4.65 & 3.47 \\
\hline 8.66 & 5.85 & 5.34 & 3.64 & 2.44 \\
\hline 7.45 & 5.34 & 3.45 & 3.34 & 2.67 \\
\hline 6.54 & 4.02 & 3.98 & 3.12 & 3.67 \\
\hline 4.67 & 3.98 & 3.45 & 3.99 & 3.56 \\
\hline
\end{tabular}




\section{Table 3b. The Market Factor + Corporate Liquidity Factor on 25 Size-BE/ME Portfolios}

Time series regressions of excess stock returns on the excess market returns $(M K T)$ and the returns on corporate liquidity (LMI) factors: July 1963 to December 2005, 510 months.

$$
R_{t}^{i}-R F_{t}=a^{i}+b^{i} M K T_{t}+c^{i} L M I_{t}+e_{t}
$$

Dependent variable: Excess returns on 25 stock portfolios formed on size and book-to-market equity

Size

quintiles

Book-to-market equity (BE/ME) quintiles

\begin{tabular}{rrrrrr} 
& \multicolumn{5}{c}{$\mathrm{a}$} \\
\cline { 2 - 6 } Small & -0.50 & 1.42 & 2.02 & 1.82 & 1.46 \\
2 & 1.02 & 1.49 & 2.08 & 1.49 & 1.06 \\
3 & 0.88 & 1.03 & 0.85 & 0.84 & 0.66 \\
4 & -0.29 & 0.42 & 0.49 & 0.20 & 0.14 \\
Big & -1.51 & -0.72 & -0.29 & -0.15 & -0.04 \\
\hline
\end{tabular}

\begin{tabular}{rrrrr}
\hline Low & 2 & 3 & 4 & High \\
\hline \multicolumn{5}{c}{$\mathrm{t}(\mathrm{a})$} \\
-0.86 & 3.54 & 5.99 & 7.90 & 9.24 \\
2.36 & 4.95 & 7.26 & 7.99 & 8.66 \\
2.38 & 3.76 & 4.65 & 4.93 & 5.08 \\
-0.86 & 1.85 & 2.60 & 1.25 & 0.97 \\
-5.67 & -3.32 & -1.43 & -0.78 & -0.25 \\
\hline
\end{tabular}

\begin{tabular}{rccccc} 
& \multicolumn{6}{c}{$\mathrm{b}$} \\
\cline { 2 - 6 } Small & 1.26 & 1.44 & 1.39 & 1.26 & 1.06 \\
2 & 1.29 & 1.30 & 1.26 & 1.19 & 1.03 \\
3 & 1.04 & 1.18 & 1.16 & 1.13 & 1.06 \\
4 & 1.05 & 1.12 & 1.13 & 1.13 & 1.07 \\
Big & 1.06 & 1.05 & 1.14 & 1.11 & 1.09 \\
\hline
\end{tabular}

\begin{tabular}{rrrrr}
\multicolumn{5}{c}{$\mathrm{t}(\mathrm{b})$} \\
\hline 9.62 & 15.82 & 18.10 & 24.02 & 29.38 \\
13.12 & 18.89 & 19.35 & 27.97 & 37.06 \\
12.37 & 18.88 & 27.90 & 29.08 & 35.46 \\
13.88 & 21.68 & 26.20 & 31.64 & 31.48 \\
17.53 & 21.22 & 24.95 & 24.83 & 29.25 \\
\hline
\end{tabular}

\begin{tabular}{rccccc} 
& \multicolumn{5}{c}{$\mathrm{c}$} \\
\cline { 2 - 6 } Small & 0.08 & 0.17 & 0.42 & 0.02 & 0.10 \\
2 & 0.11 & 0.10 & 0.18 & 0.10 & 0.29 \\
3 & 0.22 & 1.12 & 0.24 & 0.30 & 0.40 \\
4 & 0.28 & 0.36 & 0.34 & 0.38 & 0.40 \\
Big & 0.26 & 0.34 & 0.36 & 0.42 & 0.41 \\
\hline
\end{tabular}

\begin{tabular}{rrrrr}
\multicolumn{5}{c}{$\mathrm{t}(\mathrm{c})$} \\
0.49 & 1.41 & 4.12 & 0.33 & 2.19 \\
0.81 & 12.75 & 2.04 & 1.77 & 7.87 \\
1.99 & 2.06 & 4.43 & 5.93 & 10.11 \\
2.78 & 5.20 & 5.94 & 8.08 & 9.02 \\
3.24 & 5.20 & 6.00 & 7.11 & 8.35 \\
\hline & & & & \\
& & $\mathrm{s}(\mathrm{e})$ & & \\
\hline 12.91 & 8.96 & 7.57 & 5.18 & 3.54 \\
9.66 & 6.77 & 6.42 & 4.18 & 2.74 \\
8.26 & 6.15 & 4.10 & 3.83 & 2.93 \\
7.46 & 5.10 & 4.24 & 3.52 & 3.34 \\
5.97 & 4.85 & 4.48 & 4.41 & 3.66 \\
\hline
\end{tabular}

GRS test: $F$ statistic $=13.858, p$ value $<0.0001$ 


\section{Table 4. The Fama-French Three-Factor + Corporate Liquidity Factor on 25 Size-BE/ME Portfolios}

Time series regressions of excess stock returns on the excess market returns $(M K T)$, the returns on size (SMB), book-tomarket equity $(H M L)$ and the corporate liquidity $(L M I)$ factors: July 1963 to December 2005, 510 months.

$$
R_{t}^{i}-R F_{t}=a^{i}+b^{i} M K T_{t}+s^{i} S M B_{t}+h^{i} H M L_{t}+c^{i} L M I_{t}+e_{t}
$$

Dependent variable: Excess returns on 25 stock portfolios formed on size and book-to-market equity

Size

Book-to-market equity (BE/ME) quintiles

\begin{tabular}{|c|c|c|c|c|c|c|c|c|c|c|}
\hline \multirow[t]{2}{*}{ quintiles } & Low & 2 & 3 & 4 & High & Low & 2 & 3 & 4 & High \\
\hline & \multicolumn{5}{|c|}{$\mathrm{a}$} & \multicolumn{5}{|c|}{$t(a)$} \\
\hline Small & 0.56 & -0.20 & -0.19 & 0.31 & 0.89 & 1.34 & -0.84 & -0.52 & 1.11 & 4.15 \\
\hline 2 & 0.57 & 0.04 & 0.45 & 0.38 & 0.51 & 1.65 & 0.12 & 0.45 & 1.45 & 2.38 \\
\hline 3 & 0.35 & 0.22 & 0.24 & 0.28 & 0.33 & 1.36 & 0.79 & 0.82 & 1.13 & 1.82 \\
\hline 4 & 0.42 & -0.03 & -0.04 & 0.31 & 0.03 & 1.77 & -0.03 & -0.12 & 1.19 & 1.76 \\
\hline \multirow[t]{2}{*}{ Big } & 0.59 & 0.19 & 0.15 & 0.39 & 0.64 & 3.12 & 0.79 & 0.78 & 1.25 & 1.65 \\
\hline & \multicolumn{5}{|c|}{$\mathrm{b}$} & \multicolumn{5}{|c|}{$\mathrm{t}(\mathrm{b})$} \\
\hline Small & 1.16 & 1.28 & 1.20 & 1.08 & 0.97 & 9.08 & 14.57 & 16.19 & 21.56 & 25.86 \\
\hline 2 & 1.14 & 1.14 & 1.12 & 1.09 & 1.03 & 11.75 & 17.79 & 17.84 & 27.56 & 34.29 \\
\hline 3 & 0.90 & 1.05 & 1.07 & 1.06 & 1.05 & 11.15 & 18.05 & 29.41 & 28.91 & 34.41 \\
\hline 4 & 0.92 & 1.03 & 1.07 & 1.08 & 1.09 & 13.58 & 23.59 & 30.66 & 33.76 & 30.81 \\
\hline \multirow[t]{2}{*}{ Big } & 0.96 & 0.95 & 1.07 & 1.09 & 1.11 & 19.46 & 23.32 & 29.26 & 26.11 & 28.97 \\
\hline & \multicolumn{5}{|c|}{$\mathrm{s}$} & \multicolumn{5}{|c|}{$\mathrm{t}(\mathrm{s})$} \\
\hline Small & 1.79 & 1.39 & 1.21 & 0.82 & 0.26 & 10.34 & 11.73 & 12.08 & 12.10 & 5.18 \\
\hline 2 & 1.31 & 1.19 & 1.02 & 0.73 & 0.13 & 9.96 & 13.71 & 12.04 & 13.56 & 3.14 \\
\hline 3 & 1.29 & 1.08 & 0.82 & 0.63 & 0.28 & 11.87 & 13.79 & 16.65 & 12.58 & 6.88 \\
\hline 4 & 1.37 & 1.04 & 0.89 & 0.64 & 0.19 & 14.94 & 17.55 & 18.81 & 14.89 & 3.98 \\
\hline \multirow[t]{2}{*}{ Big } & 1.28 & 1.04 & 0.95 & 0.67 & 0.26 & 19.26 & 18.84 & 19.17 & 11.77 & 5.09 \\
\hline & \multicolumn{5}{|c|}{$\mathrm{h}$} & \multicolumn{5}{|c|}{$\mathrm{t}(\mathrm{h})$} \\
\hline Small & 1.10 & 0.37 & -0.01 & -0.30 & -0.23 & 4.64 & 2.25 & -0.04 & -3.18 & -3.25 \\
\hline 2 & 0.41 & 0.18 & 0.12 & 0.12 & 0.08 & 2.24 & 1.53 & 1.01 & 1.66 & 1.42 \\
\hline 3 & 0.41 & 0.24 & 0.23 & 0.20 & 0.23 & 2.72 & 2.22 & 3.35 & 2.91 & 4.13 \\
\hline 4 & 0.56 & 0.46 & 0.51 & 0.31 & 0.33 & 4.41 & 5.70 & 7.86 & 5.19 & 4.99 \\
\hline Big & 0.60 & 0.41 & 0.53 & 0.52 & 0.39 & 6.59 & 5.44 & 7.84 & 6.72 & 5.49 \\
\hline
\end{tabular}


Table 4. (continued)

\begin{tabular}{rccccc} 
& \multicolumn{7}{c}{$\mathrm{c}$} \\
\cline { 2 - 6 } Small & 0.04 & 0.37 & 0.36 & 0.39 & 0.29 \\
2 & 0.27 & 0.33 & 0.40 & 0.24 & 0.29 \\
3 & 0.38 & 0.35 & 0.36 & 0.38 & 0.29 \\
4 & 0.39 & 0.42 & 0.34 & 0.41 & 0.30 \\
Big & 0.42 & 0.36 & 0.75 & 0.35 & 0.32 \\
\hline
\end{tabular}

\begin{tabular}{lllll}
\multicolumn{7}{c}{$\mathrm{t}(\mathrm{c})$} \\
\hline 0.22 & 2.74 & 6.38 & 5.01 & 4.87 \\
1.78 & 3.37 & 4.09 & 3.89 & 6.15 \\
3.02 & 3.90 & 6.35 & 6.65 & 4.92 \\
3.64 & 6.13 & 6.18 & 8.23 & 5.40 \\
4.18 & 6.70 & 6.54 & 5.40 & 7.64 \\
\hline
\end{tabular}

\begin{tabular}{rlllll} 
& \multicolumn{5}{c}{$\mathrm{R}^{2}$} \\
\cline { 2 - 6 } Small & 0.78 & 0.88 & 0.86 & 0.82 & 0.83 \\
2 & 0.82 & 0.83 & 0.82 & 0.76 & 0.79 \\
3 & 0.82 & 0.83 & 0.76 & 0.68 & 0.75 \\
4 & 0.86 & 0.79 & 0.72 & 0.71 & 0.76 \\
Big & 0.86 & 0.78 & 0.73 & 0.69 & 0.77 \\
\hline
\end{tabular}

\begin{tabular}{ccccc}
\multicolumn{5}{c}{$\mathrm{s}(\mathrm{e})$} \\
\hline 7.61 & 3.96 & 6.71 & 4.55 & 3.42 \\
5.26 & 4.81 & 5.69 & 3.59 & 4.72 \\
5.13 & 5.43 & 3.80 & 8.34 & 5.42 \\
2.89 & 4.98 & 3.18 & 6.91 & 4.22 \\
3.23 & 7.82 & 4.72 & 4.55 & 3.77 \\
\hline
\end{tabular}

GRS test: $F$ value $=3.02$, $p$-value $=0.00$ 
Table 5. Summary Statistics on the 27 Size, BE/ME and Corporate Liquidity Sorted Portfolios

The 27 portfolios are formed from the intersection of three independent sorts of all stocks into three size, three book-to-market equity and three corporate liquidity portfolios. Column 5 through column 12 describe the characteristics of each portfolio. Average returns are reported in percentage terms. Average firm size is reported in millions of dollars.

\begin{tabular}{|c|c|c|c|c|c|c|c|c|c|c|c|c|}
\hline & Size & $B E / M E$ & $\begin{array}{l}\text { Corporate } \\
\text { Liquidity }\end{array}$ & $\begin{array}{r}\text { \#s of Firm- } \\
\text { months }\end{array}$ & $\begin{array}{r}\text { Average } \\
\text { Return }\end{array}$ & Size & $B E / M E$ & $\begin{array}{r}\text { Corporate } \\
\text { Liquidity }\end{array}$ & Leverage & $\begin{array}{l}\text { Cash } \\
\text { Flow }\end{array}$ & $\begin{array}{r}C F \\
\text { Sigma }\end{array}$ & Beta \\
\hline 1 & Small & Low & Illiquid & 24052 & 1.412 & 22.123 & 0.055 & 0.019 & 0.684 & -0.045 & 0.724 & 1.171 \\
\hline 2 & Small & Low & Medium & 25584 & 1.492 & 23.641 & 0.197 & 0.078 & 0.600 & -0.026 & 0.671 & 1.176 \\
\hline 3 & Small & Low & Liquid & 33983 & 1.884 & 23.911 & 0.297 & 0.289 & 0.409 & -0.109 & 0.528 & 1.231 \\
\hline 4 & Small & Medium & Illiquid & 30778 & 1.025 & 22.554 & 0.740 & 0.020 & 0.521 & 0.044 & 0.385 & 1.096 \\
\hline 5 & Small & Medium & Medium & 30626 & 1.992 & 22.689 & 0.742 & 0.080 & 0.458 & 0.051 & 0.341 & 1.101 \\
\hline 6 & Small & Medium & Liquid & 36018 & 1.472 & 21.445 & 0.743 & 0.293 & 0.286 & 0.035 & 0.399 & 1.128 \\
\hline 7 & Small & High & Illiquid & 69681 & -0.504 & 20.512 & 3.089 & 0.020 & 0.476 & 0.040 & 0.159 & 1.078 \\
\hline 8 & Small & High & Medium & 63396 & 0.035 & 19.743 & 6.443 & 0.078 & 0.440 & 0.042 & 0.233 & 1.053 \\
\hline 9 & Small & High & Liquid & 51481 & -0.022 & 19.292 & 4.288 & 0.290 & 0.312 & 0.042 & 0.318 & 1.054 \\
\hline 10 & Medium & Low & Illiquid & 22395 & 2.422 & 117.614 & 0.260 & 0.021 & 0.566 & 0.042 & 0.435 & 1.152 \\
\hline 11 & Medium & Low & Medium & 29616 & 2.524 & 125.378 & 0.279 & 0.080 & 0.490 & 0.044 & 0.615 & 1.143 \\
\hline 12 & Medium & Low & Liquid & 60462 & 2.956 & 117.223 & 0.333 & 0.311 & 0.294 & 0.018 & 0.456 & 1.128 \\
\hline 13 & Medium & Medium & Illiquid & 41064 & 1.761 & 128.934 & 0.739 & 0.021 & 0.478 & 0.074 & 0.202 & 1.041 \\
\hline 14 & Medium & Medium & Medium & 44352 & 1.860 & 127.538 & 0.732 & 0.078 & 0.421 & 0.080 & 0.262 & 1.060 \\
\hline 15 & Medium & Medium & Liquid & 48316 & 1.694 & 121.778 & 0.711 & 0.252 & 0.322 & 0.069 & 0.255 & 1.074 \\
\hline 16 & Medium & High & Illiquid & 48741 & 0.453 & 110.344 & 2.166 & 0.022 & 0.459 & 0.058 & 0.131 & 1.027 \\
\hline 17 & Medium & High & Medium & 42817 & 0.795 & 112.878 & 3.195 & 0.078 & 0.427 & 0.063 & 0.149 & 1.068 \\
\hline 18 & Medium & High & Liquid & 28059 & -0.022 & 100.543 & 1.841 & 0.230 & 0.344 & 0.072 & 0.147 & 1.011 \\
\hline 19 & Big & Low & Illiquid & 44989 & 2.121 & 3943.539 & 0.337 & 0.021 & 0.474 & 0.096 & 0.311 & 1.014 \\
\hline 20 & Big & Low & Medium & 54641 & 2.383 & 4631.514 & 0.336 & 0.079 & 0.421 & 0.101 & 0.426 & 1.011 \\
\hline 21 & Big & Low & Liquid & 70435 & 2.905 & 2034.118 & 0.299 & 0.251 & 0.300 & 0.109 & 0.458 & 1.013 \\
\hline 22 & Big & Medium & Illiquid & 56721 & 1.267 & 2040.094 & 0.709 & 0.021 & 0.444 & 0.080 & 0.175 & 0.973 \\
\hline 23 & Big & Medium & Medium & 50040 & 1.608 & 2379.624 & 0.708 & 0.075 & 0.392 & 0.085 & 0.164 & 0.981 \\
\hline 24 & Big & Medium & Liquid & 28057 & 1.703 & 1413.237 & 0.698 & 0.216 & 0.355 & 0.086 & 0.197 & 1.056 \\
\hline 25 & Big & High & Illiquid & 27654 & 0.414 & 1658.489 & 1.630 & 0.021 & 0.430 & 0.065 & 0.113 & 1.029 \\
\hline 26 & Big & High & Medium & 24849 & 0.705 & 1394.633 & 2.367 & 0.075 & 0.390 & 0.072 & 0.105 & 1.004 \\
\hline 27 & Big & High & Liquid & 9130 & 0.696 & 1144.567 & 2.124 & 0.221 & 0.401 & 0.074 & 0.120 & 0.987 \\
\hline
\end{tabular}




\section{Table 6. The Fama-French Three-Factor Model on 27 Size-BE/ME-Corporate Liquidity Portfolios}

Time series regressions of excess stock returns on the excess market returns $(M K T)$, the returns on size $(S M B)$ and book-to-market equity $(H M L)$ factors: July 1963 to December 2005, 510 months.

$$
R_{t}^{i}-R F_{t}=a^{i}+b^{i} M K T_{t}+s^{i} S M B_{t}+h^{i} H M L_{t}+e_{t}
$$

Dependent variable: Excess returns on 27 stock portfolios formed on size, book-to-market equity and corporate liquidity.

Corporate Liquidity Tertiles - Illiquid

\begin{tabular}{|c|c|c|c|c|c|c|}
\hline \multirow{2}{*}{$\begin{array}{r}\text { Size } \\
\text { Tertiles }\end{array}$} & \multicolumn{6}{|c|}{ Book-to-market Equity (BE/ME) Tertiles } \\
\hline & Low & Medium & High & Low & Medium & High \\
\hline & \multicolumn{3}{|c|}{$\mathrm{a}$} & \multicolumn{3}{|c|}{$\mathrm{t}(\mathrm{a})$} \\
\hline Small & -0.12 & 0.11 & 0.23 & -0.35 & 0.52 & 1.18 \\
\hline Medium & -0.84 & -0.19 & -0.24 & -2.60 & -0.76 & -0.69 \\
\hline \multirow[t]{2}{*}{ Big } & 0.26 & 0.07 & 0.03 & 1.79 & 0.43 & 0.19 \\
\hline & \multicolumn{3}{|c|}{$\mathrm{b}$} & \multicolumn{3}{|c|}{$\mathrm{t}(\mathrm{b})$} \\
\hline Small & 1.27 & 1.01 & 0.98 & 12.87 & 15.18 & 21.77 \\
\hline Medium & 1.25 & 1.08 & 1.04 & 20.04 & 27.87 & 31.27 \\
\hline \multirow[t]{2}{*}{ Big } & 1.11 & 1.10 & 1.16 & 28.25 & 34.07 & 29.54 \\
\hline & \multicolumn{3}{|c|}{ s } & \multicolumn{3}{|c|}{$\mathrm{t}(\mathrm{s})$} \\
\hline Small & 1.45 & 1.15 & $\overline{1.22}$ & 11.11 & 13.15 & 20.43 \\
\hline Medium & 0.86 & 0.88 & 0.77 & 10.51 & 17.15 & 17.45 \\
\hline \multirow[t]{2}{*}{ Big } & 0.25 & 0.31 & 0.36 & 4.89 & 7.29 & 6.86 \\
\hline & \multicolumn{3}{|c|}{$\mathrm{h}$} & \multicolumn{3}{|c|}{$\mathrm{t}(\mathrm{h})$} \\
\hline Small & 0.91 & 0.54 & 0.86 & 6.06 & 5.30 & 12.54 \\
\hline Medium & 0.47 & 0.57 & 0.73 & 4.96 & 9.60 & 14.45 \\
\hline \multirow[t]{2}{*}{ Big } & 0.25 & 0.52 & 0.70 & 4.24 & 10.61 & 11.75 \\
\hline & \multicolumn{3}{|c|}{$\mathrm{R}^{2}$} & \multicolumn{3}{|c|}{$\mathrm{s}(\mathrm{e})$} \\
\hline Small & 0.73 & 0.69 & $\overline{0.82}$ & 5.76 & 4.84 & 3.34 \\
\hline Medium & 0.74 & 0.64 & 0.61 & 4.43 & 4.71 & 4.85 \\
\hline Big & 0.82 & 0.78 & 0.67 & 2.07 & 3.64 & 3.78 \\
\hline
\end{tabular}


Table 6. (continued)

Corporate Liquidity Tertiles -Medium

\begin{tabular}{|c|c|c|c|c|c|c|}
\hline \multirow{2}{*}{$\begin{array}{r}\text { Size } \\
\text { Tertiles }\end{array}$} & \multicolumn{6}{|c|}{ Book-to-market Equity (BE/ME) Tertiles } \\
\hline & Low & Medium & High & Low & Medium & High \\
\hline & \multicolumn{3}{|c|}{$\mathrm{a}$} & \multicolumn{3}{|c|}{$\mathrm{t}(\mathrm{a})$} \\
\hline Small & -0.29 & 0.32 & 0.29 & -0.89 & 1.02 & 1.28 \\
\hline Medium & -0.56 & 0.18 & -0.34 & -1.99 & 0.68 & -1.24 \\
\hline \multirow[t]{2}{*}{ Big } & 0.25 & -0.15 & 0.12 & 1.59 & -0.56 & 0.56 \\
\hline & \multicolumn{3}{|c|}{$\mathrm{b}$} & \multicolumn{3}{|c|}{$\mathrm{t}(\mathrm{b})$} \\
\hline Small & 0.97 & 0.91 & 1.02 & 10.70 & 11.72 & 19.31 \\
\hline Medium & 1.12 & 1.10 & 1.12 & 15.16 & 26.29 & 28.41 \\
\hline \multirow[t]{2}{*}{ Big } & 1.02 & 1.07 & 1.07 & 27.67 & 32.53 & 25.01 \\
\hline & \multicolumn{3}{|c|}{$\mathrm{s}$} & \multicolumn{3}{|c|}{$\mathrm{t}(\mathrm{s})$} \\
\hline Small & 1.60 & 1.21 & 1.14 & 13.42 & 11.87 & 16.44 \\
\hline Medium & 0.92 & 0.78 & 0.87 & 9.48 & 14.28 & 16.89 \\
\hline \multirow[t]{2}{*}{ Big } & 0.26 & 0.28 & 0.40 & 5.28 & 6.39 & 7.07 \\
\hline & \multicolumn{3}{|c|}{$\mathrm{h}$} & \multicolumn{3}{|c|}{$\mathrm{t}(\mathrm{h})$} \\
\hline Small & 0.58 & 0.62 & 0.79 & 4.22 & 5.27 & 9.83 \\
\hline Medium & 0.57 & 0.47 & 0.71 & 5.04 & 7.39 & 11.93 \\
\hline \multirow[t]{2}{*}{ Big } & 0.05 & 0.42 & 0.63 & 0.84 & 8.36 & 9.61 \\
\hline & \multicolumn{3}{|c|}{$\mathrm{R}^{2}$} & \multicolumn{3}{|c|}{$\mathrm{s}(\mathrm{e})$} \\
\hline Small & 0.71 & 0.73 & 0.73 & 5.99 & 4.12 & 3.25 \\
\hline Medium & 0.72 & 0.64 & 0.67 & 4.34 & 4.89 & 3.59 \\
\hline Big & 0.86 & 0.78 & 0.70 & 2.02 & 3.01 & 3.67 \\
\hline
\end{tabular}


Table 6. (continued)

Corporate Liquidity Tertiles - Liquid

\begin{tabular}{|c|c|c|c|c|c|c|}
\hline \multirow{2}{*}{$\begin{array}{r}\text { Size } \\
\text { Tertiles }\end{array}$} & \multicolumn{6}{|c|}{ Book-to-market Equity (BE/ME) Tertiles } \\
\hline & Low & Medium & High & Low & Medium & High \\
\hline & \multicolumn{3}{|c|}{$\mathrm{a}$} & \multicolumn{3}{|c|}{$\mathrm{t}(\mathrm{a})$} \\
\hline Small & 0.17 & 0.29 & 0.56 & 0.42 & 0.69 & 2.21 \\
\hline Medium & -0.04 & 0.37 & -0.09 & -0.23 & 1.14 & -0.39 \\
\hline \multirow[t]{2}{*}{ Big } & 0.89 & 0.51 & 0.09 & 3.46 & 1.91 & 0.67 \\
\hline & \multicolumn{3}{|c|}{$\mathrm{b}$} & \multicolumn{3}{|c|}{$\mathrm{t}(\mathrm{b})$} \\
\hline Small & 1.16 & 1.10 & 0.94 & 11.24 & 13.57 & 15.84 \\
\hline Medium & 1.18 & 1.11 & 0.92 & 16.13 & 21.79 & 16.33 \\
\hline \multirow[t]{2}{*}{ Big } & 0.89 & 1.07 & 0.93 & 13.19 & 18.22 & 19.28 \\
\hline & \multicolumn{3}{|c|}{$\mathrm{S}$} & \multicolumn{3}{|c|}{$\mathrm{t}(\mathrm{s})$} \\
\hline Small & 1.14 & 0.95 & 1.02 & 8.35 & 8.92 & 13.06 \\
\hline Medium & 0.95 & 0.76 & 0.84 & 9.91 & 11.31 & 11.34 \\
\hline \multirow[t]{2}{*}{ Big } & 0.73 & 0.34 & 0.28 & 8.20 & 4.44 & 4.39 \\
\hline & \multicolumn{3}{|c|}{$\mathrm{h}$} & \multicolumn{3}{|c|}{$\mathrm{t}(\mathrm{h})$} \\
\hline Small & 0.54 & 0.63 & 0.83 & 3.42 & 5.11 & 9.16 \\
\hline Medium & 0.30 & 0.35 & 0.67 & 2.72 & 4.57 & 7.80 \\
\hline \multirow[t]{2}{*}{ Big } & -0.33 & 0.18 & 0.48 & -3.21 & 2.05 & 6.55 \\
\hline & \multicolumn{3}{|c|}{$\mathrm{R}^{2}$} & \multicolumn{3}{|c|}{$\mathrm{s}(\mathrm{e})$} \\
\hline Small & 0.80 & 0.75 & 0.77 & 3.92 & 4.82 & 3.80 \\
\hline Medium & 0.76 & 0.73 & 0.62 & 4.77 & 4.88 & 4.91 \\
\hline Big & 0.83 & 0.80 & 0.61 & 3.30 & 3.62 & 4.91 \\
\hline
\end{tabular}

GRS test: $F$ value $=1.43, p$-value $=0.09$ 


\section{Table 7. The Fama-French Three-Factor + Corporate Liquidity Factor on 27 Size-BE/ME-Corporate Liquidity Portfolios}

Time series regressions of excess stock returns on the excess market returns $(M K T)$, the returns on size $(S M B)$, book-to-market equity $(H M L)$ and the corporate liquidity $(L M I)$ factors: July 1963 to December 2005, 510 months.

$$
R_{t}^{i}-R F_{t}=a^{i}+b^{i} M K T_{t}+s^{i} S M B_{t}+h^{i} H M L_{t}+c^{i} L M I_{t}+e_{t}
$$

Dependent variable: Excess returns on 27 stock portfolios formed on size, book-to-market equity and corporate liquidity.

Corporate Liquidity Tertiles - Illiquid

\begin{tabular}{|c|c|c|c|c|c|c|}
\hline \multirow{2}{*}{$\begin{array}{r}\text { Size } \\
\text { Tertiles }\end{array}$} & \multicolumn{6}{|c|}{ Book-to-market Equity (BE/ME) Tertiles } \\
\hline & Low & Medium & High & Low & Medium & High \\
\hline & \multicolumn{3}{|c|}{$\mathrm{a}$} & \multicolumn{3}{|c|}{$\mathrm{t}(\mathrm{a})$} \\
\hline Small & 0.16 & 0.31 & 0.57 & 0.44 & 0.93 & 2.22 \\
\hline Medium & -0.05 & 0.33 & 0.12 & -0.15 & 1.04 & 0.41 \\
\hline \multirow[t]{2}{*}{ Big } & 0.90 & 0.17 & 0.17 & 3.63 & 0.70 & 0.54 \\
\hline & \multicolumn{3}{|c|}{$\mathrm{b}$} & \multicolumn{3}{|c|}{$t(b)$} \\
\hline Small & 1.26 & 1.00 & 0.97 & 12.84 & 15.14 & 22.19 \\
\hline Medium & 1.23 & 1.07 & 1.03 & 20.64 & 28.99 & 32.79 \\
\hline \multirow[t]{2}{*}{ Big } & 1.10 & 1.08 & 1.15 & 30.37 & 36.41 & 30.70 \\
\hline & \multicolumn{3}{|c|}{$\mathrm{S}$} & \multicolumn{3}{|c|}{$\mathrm{t}(\mathrm{s})$} \\
\hline Small & 1.54 & 1.20 & 1.30 & 11.63 & 13.43 & 21.86 \\
\hline Medium & 0.99 & 0.96 & 0.85 & 12.33 & 19.33 & 19.89 \\
\hline \multirow[t]{2}{*}{ Big } & 0.37 & 0.40 & 0.44 & 7.53 & 9.88 & 8.75 \\
\hline & \multicolumn{3}{|c|}{$\mathrm{h}$} & \multicolumn{3}{|c|}{$\mathrm{t}(\mathrm{h})$} \\
\hline Small & 0.58 & 0.36 & 0.59 & 3.19 & 2.93 & 7.22 \\
\hline Medium & 0.02 & 0.27 & 0.46 & 0.22 & 3.93 & 7.88 \\
\hline \multirow[t]{2}{*}{ Big } & -0.13 & 0.22 & 0.41 & -1.95 & 3.96 & 5.84 \\
\hline & \multicolumn{3}{|c|}{$\mathrm{c}$} & \multicolumn{3}{|c|}{$t(c)$} \\
\hline Small & -0.48 & -0.26 & -0.40 & -3.17 & -2.49 & -5.86 \\
\hline Medium & -0.65 & -0.44 & -0.40 & -7.02 & -7.58 & -8.11 \\
\hline \multirow[t]{2}{*}{ Big } & -0.56 & -0.44 & -0.43 & -9.99 & -9.45 & -7.43 \\
\hline & \multicolumn{3}{|c|}{$\mathrm{R}^{2}$} & \multicolumn{3}{|c|}{$\mathrm{s}(\mathrm{e})$} \\
\hline Small & 0.77 & 0.69 & 0.83 & 5.29 & 3.91 & 3.10 \\
\hline Medium & 0.72 & 0.66 & 0.63 & 4.37 & 4.77 & 3.76 \\
\hline Big & 0.83 & 0.78 & 0.68 & 2.08 & 2.87 & 3.66 \\
\hline
\end{tabular}


Table 7. (continued)

Corporate Liquidity Tertiles - Medium

\begin{tabular}{|c|c|c|c|c|c|c|}
\hline \multirow{2}{*}{$\begin{array}{r}\text { Size } \\
\text { Tertiles }\end{array}$} & \multicolumn{6}{|c|}{ Book-to-market Equity (BE/ME) Tertiles } \\
\hline & Low & Medium & High & Low & Medium & High \\
\hline & \multicolumn{3}{|c|}{$\mathrm{a}$} & \multicolumn{3}{|c|}{$\mathrm{t}(\mathrm{a})$} \\
\hline Small & 0.21 & 0.17 & 0.35 & 0.53 & -0.59 & 1.42 \\
\hline Medium & -0.21 & -0.20 & -0.18 & -1.45 & 0.73 & -0.61 \\
\hline \multirow[t]{2}{*}{ Big } & 0.29 & 0.14 & 0.09 & 1.28 & 0.70 & 0.37 \\
\hline & \multicolumn{3}{|c|}{$\mathrm{b}$} & \multicolumn{3}{|c|}{$\mathrm{t}(\mathrm{b})$} \\
\hline Small & 0.96 & 0.90 & 1.01 & 10.66 & 11.68 & 19.54 \\
\hline Medium & 1.10 & 1.08 & 1.11 & 15.59 & 27.36 & 29.37 \\
\hline \multirow[t]{2}{*}{ Big } & 1.01 & 1.07 & 1.07 & 28.35 & 32.88 & 25.23 \\
\hline & \multicolumn{3}{|c|}{$\underline{s}$} & \multicolumn{3}{|c|}{$\mathrm{t}(\mathrm{s})$} \\
\hline Small & 1.62 & 1.28 & 1.23 & 13.21 & 12.33 & 17.56 \\
\hline Medium & 1.09 & 0.88 & 0.96 & 11.40 & 16.41 & 18.79 \\
\hline \multirow[t]{2}{*}{ Big } & 0.33 & 0.32 & 0.46 & 6.76 & 7.36 & 8.02 \\
\hline & \multicolumn{3}{|c|}{$\underline{h}$} & \multicolumn{3}{|c|}{$\mathrm{t}(\mathrm{h})$} \\
\hline Small & 0.51 & 0.37 & 0.51 & 3.03 & 2.62 & 5.30 \\
\hline Medium & 0.01 & 0.15 & 0.43 & 0.11 & 1.99 & 6.20 \\
\hline \multirow[t]{2}{*}{ Big } & -0.19 & 0.27 & 0.43 & -2.82 & 4.44 & 5.49 \\
\hline & \multicolumn{3}{|c|}{ c } & \multicolumn{3}{|c|}{$\mathrm{t}(\mathrm{c})$} \\
\hline Small & -0.11 & 0.28 & 0.33 & -0.75 & 2.19 & 3.59 \\
\hline Medium & 0.22 & -0.15 & 0.35 & 1.97 & -1.87 & 4.01 \\
\hline \multirow[t]{2}{*}{$\mathrm{Big}$} & 0.34 & -0.22 & 0.29 & 6.15 & -4.41 & 4.38 \\
\hline & \multicolumn{3}{|c|}{$\mathrm{R}^{2}$} & \multicolumn{3}{|c|}{$\mathrm{s}(\mathrm{e})$} \\
\hline Small & 0.72 & 0.73 & 0.78 & 5.16 & 3.82 & 3.16 \\
\hline Medium & 0.75 & 0.69 & 0.68 & 3.84 & 4.59 & 4.88 \\
\hline Big & 0.87 & 0.77 & 0.72 & 2.06 & 3.91 & 4.56 \\
\hline
\end{tabular}


Table 7. (continued)

Corporate Liquidity Tertiles - Liquid

\begin{tabular}{|c|c|c|c|c|c|c|}
\hline \multirow{2}{*}{$\begin{array}{r}\text { Size } \\
\text { Tertiles }\end{array}$} & \multicolumn{6}{|c|}{ Book-to-market Equity (BE/ME) Tertiles } \\
\hline & Low & Medium & High & Low & Medium & High \\
\hline & \multicolumn{3}{|c|}{$\mathrm{a}$} & \multicolumn{3}{|c|}{$\mathrm{t}(\mathrm{a})$} \\
\hline Small & -0.47 & 0.25 & 0.17 & -1.16 & 0.86 & 0.82 \\
\hline Medium & -0.57 & -0.26 & -0.36 & -1.67 & -0.93 & -1.35 \\
\hline \multirow[t]{2}{*}{ Big } & 0.26 & 0.15 & 0.08 & 1.59 & 0.81 & 0.36 \\
\hline & \multicolumn{3}{|c|}{$\mathrm{b}$} & \multicolumn{3}{|c|}{$\mathrm{t}(\mathrm{b})$} \\
\hline Small & 1.16 & 1.10 & 0.94 & 11.18 & 13.52 & 15.86 \\
\hline Medium & 1.17 & 1.10 & 0.91 & 16.08 & 21.75 & 16.38 \\
\hline \multirow[t]{2}{*}{ Big } & 0.91 & 1.07 & 0.92 & 13.89 & 18.19 & 19.51 \\
\hline & \multicolumn{3}{|c|}{$\mathrm{s}$} & \multicolumn{3}{|c|}{$\mathrm{t}(\mathrm{s})$} \\
\hline Small & 1.18 & 1.01 & 1.09 & 8.44 & 9.22 & 13.68 \\
\hline Medium & 1.00 & 0.79 & 0.91 & 10.12 & 11.46 & 12.12 \\
\hline \multirow[t]{2}{*}{ Big } & 0.60 & 0.34 & 0.35 & 6.80 & 4.29 & 5.56 \\
\hline & \multicolumn{3}{|c|}{$\mathrm{h}$} & \multicolumn{3}{|c|}{$\mathrm{t}(\mathrm{h})$} \\
\hline Small & 0.39 & 0.44 & 0.61 & 2.03 & 2.94 & 5.52 \\
\hline Medium & 0.15 & 0.25 & 0.43 & 1.10 & 2.68 & 4.18 \\
\hline \multirow[t]{2}{*}{ Big } & 0.10 & 0.19 & 0.22 & 0.80 & 1.72 & 2.56 \\
\hline & \multicolumn{3}{|c|}{ c } & \multicolumn{3}{|c|}{$t(c)$} \\
\hline Small & 0.22 & 0.36 & 0.41 & 1.34 & 3.00 & 5.12 \\
\hline Medium & 0.81 & 0.47 & 0.42 & 7.35 & 7.63 & 6.97 \\
\hline \multirow[t]{2}{*}{ Big } & 0.62 & -0.01 & 0.37 & 6.14 & -0.07 & 5.10 \\
\hline & \multicolumn{3}{|c|}{$\mathrm{R}^{2}$} & \multicolumn{3}{|c|}{$\mathrm{s}(\mathrm{e})$} \\
\hline Small & 0.82 & 0.80 & 0.82 & 3.89 & 3.83 & 3.01 \\
\hline Medium & 0.79 & 0.78 & 0.69 & 4.67 & 4.79 & 4.41 \\
\hline Big & 0.84 & 0.83 & 0.64 & 2.89 & 3.01 & 4.98 \\
\hline
\end{tabular}

GRS test $: F$ value $=1.31, p$-value $=0.18$ 


\section{Table 8. CAPM and Multifactor Models on 10 Corporate Liquidity Portfolios}

This table presents the results of asset pricing models for the 10 corporate liquidity portfolios. Each June, the sample is sorted into 10 portfolios based on the firm's corporate liquidity. Then CAPM, Fama-French three-factor model as well as the four-factor model are estimated for the 10 corporate liquidity portfolios during the period July 1963 to December 2005, 510 months.

Panel A. CAPM: $R_{t}^{i}-R F_{t}=a^{i}+b^{i} M K T_{t}+e_{t}^{i}$

\begin{tabular}{|c|c|c|c|c|c|c|c|c|c|c|}
\hline & Illiquid 1 & 2 & 3 & 4 & 5 & 6 & 7 & 8 & 9 & Liquid 10 \\
\hline \multirow[t]{2}{*}{$a$} & -0.670 & 0.377 & -0.155 & 0.470 & -0.121 & -0.843 & -0.063 & 0.306 & -0.325 & -0.153 \\
\hline & $(-0.891)$ & $(0.601)$ & $(-0.248)$ & $(0.719)$ & $(-0.205)$ & $(-1.387)$ & $(-0.092)$ & $(0.491)$ & $(-0.437)$ & $(-0.216)$ \\
\hline \multirow[t]{2}{*}{$M K T$} & 1.158 & 1.217 & 1.281 & 1.367 & 1.327 & 1.356 & 1.506 & 1.532 & 1.656 & 1.719 \\
\hline & $(8.231)^{* * *}$ & $(8.937)^{* * *}$ & $(8.758)^{* * *}$ & $(8.121)^{* * *}$ & $(8.590)^{* * *}$ & $(9.703)^{* * *}$ & $(11.442)^{* * *}$ & $(10.904)^{* * *}$ & $(9.938)^{* * *}$ & $(10.825)^{* * *}$ \\
\hline$R^{2}$ & 0.111 & 0.184 & 0.114 & 0.127 & 0.199 & 0.132 & 0.123 & 0.152 & 0.158 & 0.182 \\
\hline
\end{tabular}

GRS test: $\quad$ F statistic $=0.44$, p-value $=0.93$

Panel B. Fama-French Three-Factor Model: $R_{t}^{i}-R F_{t}=a^{i}+b^{i} M K T_{t}+s^{i} S M B_{t}+h^{i} H M L_{t}+e_{t}^{i}$

\begin{tabular}{|c|c|c|c|c|c|c|c|c|c|c|}
\hline & Illiquid 1 & 2 & 3 & 4 & 5 & 6 & 7 & 8 & 9 & Liquid 10 \\
\hline \multirow[t]{2}{*}{$a$} & -1.548 & 0.327 & -0.370 & 0.118 & -0.451 & -1.240 & -0.476 & 0.219 & 0.001 & -0.053 \\
\hline & $(-2.066)$ & $(0.516)$ & $(-0.594)$ & $(0.178)$ & $(-0.768)$ & $(-2.025)^{* * *}$ & $(-0.692)$ & $(0.352)$ & $(0.002)$ & $(-0.075)$ \\
\hline \multirow[t]{2}{*}{$M K T$} & 0.899 & 1.070 & 1.146 & 1.157 & 1.155 & 1.209 & 1.312 & 1.318 & 1.359 & 1.468 \\
\hline & $(5.905)^{* * *}$ & $(7.041)^{* * *}$ & $(7.639)^{* * *}$ & $(6.870)^{* * *}$ & $(6.424)^{* * *}$ & $(7.463)^{* * *}$ & $(7.624)^{* * *}$ & $(8.487)^{* * *}$ & $(9.454)^{* * *}$ & $(8.003)^{* * *}$ \\
\hline \multirow[t]{2}{*}{$S M B$} & 1.085 & 0.852 & 1.283 & 0.838 & 1.075 & 0.912 & 1.294 & 1.174 & 1.262 & 1.300 \\
\hline & $(4.493)^{* * *}$ & $(4.166)^{* * *}$ & $(6.401)^{* * *}$ & $(3.931)^{* * *}$ & $(5.685)^{* * *}$ & $(4.620)^{* * *}$ & $(5.838)^{* * *}$ & $(5.870)^{* * *}$ & $(5.329)^{* * *}$ & $(5.740)^{* * *}$ \\
\hline \multirow[t]{2}{*}{$H M L$} & 1.262 & -0.106 & 0.079 & 0.414 & 0.324 & 0.475 & 0.416 & -0.114 & -0.844 & -0.464 \\
\hline & $(4.515)^{* * *}$ & $(-0.450)$ & $(0.340)$ & (1.678) & $(1.478)$ & $(2.080)^{* *}$ & (1.624) & $(-0.491)$ & $(-3.080)^{* * *}$ & $(-1.770)^{*}$ \\
\hline$R^{2}$ & 0.166 & 0.213 & 0.179 & 0.154 & 0.246 & 0.168 & 0.177 & 0.208 & 0.225 & 0.241 \\
\hline
\end{tabular}


Table 8. (continued)

Panel C. Fama-French Three-Factor + LMI: $R_{t}^{i}-R F_{t}=a^{i}+b^{i} M K T_{t}+s^{i} S M B_{t}+h^{i} H M L_{t}+c^{i} L M I_{t}+e_{t}$

\begin{tabular}{|c|c|c|c|c|c|c|c|c|c|c|}
\hline & Illiquid 1 & 2 & 3 & 4 & 5 & 6 & 7 & 8 & 9 & Liquid 10 \\
\hline$a$ & -0.694 & 0.504 & 0.439 & 0.524 & 0.081 & -0.744 & -0.568 & -0.413 & -0.804 & -0.243 \\
\hline & $(-0.868)$ & $(0.738)$ & $(0.662)$ & $(0.738)$ & $(0.129)$ & $(-1.133)$ & $(-0.767)$ & $(-0.622)$ & $(-1.025)$ & $(-0.321)$ \\
\hline$M K T$ & $\begin{array}{l}0.879 \\
(5.820)^{* * *}\end{array}$ & $\begin{array}{l}1.086 \\
(7.179)^{* * *}\end{array}$ & $\begin{array}{l}1.199 \\
(7.403)^{* * *}\end{array}$ & $\begin{array}{l}1.133 \\
(7.572)^{* * *}\end{array}$ & $\begin{array}{l}1.159 \\
(6.872)^{* * *}\end{array}$ & $\begin{array}{l}1.176 \\
(6.574)^{* * *}\end{array}$ & $\begin{array}{l}1.316 \\
(7.642)^{* * *}\end{array}$ & $\begin{array}{l}1.314 \\
(8.447)^{* * *}\end{array}$ & $\begin{array}{l}1.345 \\
(9.390)^{* * *}\end{array}$ & $\begin{array}{l}1.446 \\
(7.934)^{* * *}\end{array}$ \\
\hline$S M B$ & $\begin{array}{l}1.253 \\
(5.081)^{* * *}\end{array}$ & $\begin{array}{l}0.886 \\
(4.213)^{* * *}\end{array}$ & $\begin{array}{l}1.443 \\
(7.062)^{* * *}\end{array}$ & $\begin{array}{l}0.918 \\
(4.192)^{* * *}\end{array}$ & $\begin{array}{l}1.180 \\
(6.089)^{* * *}\end{array}$ & $\begin{array}{l}1.010 \\
(4.988)^{* * *}\end{array}$ & $\begin{array}{l}1.276 \\
(5.590)^{* * *}\end{array}$ & $\begin{array}{l}1.050 \\
(5.127)^{* * *}\end{array}$ & $\begin{array}{l}1.102 \\
(4.555)^{* * *}\end{array}$ & $\begin{array}{l}1.262 \\
(5.415)^{* * *}\end{array}$ \\
\hline$H M L$ & $\begin{array}{l}0.697 \\
(2.056)^{* *}\end{array}$ & $\begin{array}{l}-0.223 \\
(-0.772)\end{array}$ & $\begin{array}{l}-0.456 \\
(-1.625)\end{array}$ & $\begin{array}{l}0.145 \\
(0.483)\end{array}$ & $\begin{array}{l}-0.028 \\
(-0.106)\end{array}$ & $\begin{array}{l}0.147 \\
(0.528)\end{array}$ & $\begin{array}{l}0.477 \\
(1.521)\end{array}$ & $\begin{array}{l}0.304 \\
(1.082)\end{array}$ & $\begin{array}{l}-0.311 \\
(-0.935)\end{array}$ & $\begin{array}{l}-0.338 \\
(-1.056)\end{array}$ \\
\hline$L M I$ & $\begin{array}{l}-0.824 \\
(-2.904)^{* * *}\end{array}$ & $\begin{array}{l}-0.171 \\
(-0.704)\end{array}$ & $\begin{array}{l}-0.781 \\
(-3.320)^{* * *}\end{array}$ & $\begin{array}{l}-0.392 \\
(-1.555)\end{array}$ & $\begin{array}{l}-0.513 \\
(-2.301)^{* *}\end{array}$ & $\begin{array}{l}-0.479 \\
(-2.056)^{* *}\end{array}$ & $\begin{array}{l}0.088 \\
(0.336)\end{array}$ & $\begin{array}{l}0.610 \\
(2.589)^{* * *}\end{array}$ & $\begin{array}{l}0.778 \\
(2.792)^{* * *}\end{array}$ & $\begin{array}{l}0.184 \\
(0.684)\end{array}$ \\
\hline$R^{2}$ & 0.180 & 0.213 & 0.196 & 0.158 & 0.254 & 0.175 & 0.177 & 0.218 & 0.236 & 0.242 \\
\hline
\end{tabular}


Table 9. The Fama-French Three-Factor Model on 25 Size -Corporate Liquidity Portfolios

Time series regressions of excess stock returns on the excess market returns $(M K T)$ and the returns on size $(S M B)$ and book-to-market equity $(H M L)$ factors: July 1963 to December 2005, 510 months

$$
R_{t}^{i}-R F_{t}=a^{i}+b^{i} M K T_{t}+s^{i} S M B_{t}+h^{i} H M L_{t}+e_{t}
$$

Dependent variable: Excess returns on 25 portfolios subsequently sorted by size and corporate liquidity

Size

\begin{tabular}{|c|c|c|c|c|c|}
\hline \multirow[t]{2}{*}{ quintiles } & Illiquid & 2 & 3 & 4 & Liquid \\
\hline & \multicolumn{5}{|c|}{$\mathrm{a}$} \\
\hline Small & -0.49 & -0.32 & -0.14 & 0.30 & 0.79 \\
\hline 2 & -0.48 & -0.28 & -0.09 & 0.40 & 0.83 \\
\hline 3 & -0.47 & -0.28 & -0.03 & 0.40 & 0.85 \\
\hline 4 & -0.49 & -0.28 & -0.06 & 0.37 & 0.87 \\
\hline Big & -0.47 & -0.34 & -0.05 & 0.30 & 0.83 \\
\hline
\end{tabular}

\begin{tabular}{rccccc} 
& \multicolumn{5}{c}{$\mathrm{b}$} \\
\cline { 2 - 6 } Small & 1.05 & 1.07 & 1.06 & 1.09 & 1.12 \\
2 & 1.06 & 1.07 & 1.08 & 1.10 & 1.11 \\
3 & 1.05 & 1.06 & 1.08 & 1.09 & 1.10 \\
4 & 1.04 & 1.06 & 1.07 & 1.07 & 1.10 \\
Big & 1.06 & 1.07 & 1.06 & 1.07 & 1.08 \\
\hline
\end{tabular}

\begin{tabular}{rccccc} 
& \multicolumn{5}{c}{$\mathrm{s}$} \\
\cline { 2 - 6 } Small & 0.89 & 0.85 & 0.88 & 0.93 & 1.11 \\
2 & 0.88 & 0.86 & 0.88 & 0.98 & 1.15 \\
3 & 0.88 & 0.86 & 0.92 & 1.00 & 1.18 \\
4 & 0.88 & 0.85 & 0.92 & 1.00 & 1.20 \\
Big & 0.88 & 0.86 & 0.91 & 0.98 & 1.16 \\
\hline
\end{tabular}

\begin{tabular}{rrrrrr} 
& \multicolumn{5}{c}{$\mathrm{h}$} \\
\cline { 2 - 6 } Small & 0.54 & 0.46 & 0.36 & 0.10 & -0.31 \\
2 & 0.53 & 0.45 & 0.30 & 0.04 & -0.37 \\
3 & 0.53 & 0.45 & 0.25 & -0.03 & -0.40 \\
4 & 0.54 & 0.45 & 0.28 & -0.02 & -0.40 \\
Big & 0.54 & 0.45 & 0.31 & 0.06 & -0.32 \\
\hline
\end{tabular}

$\mathrm{R}^{2}$

\begin{tabular}{rlllll} 
& \multicolumn{5}{c}{$\mathrm{R}^{2}$} \\
\cline { 2 - 6 } Small & 0.90 & 0.92 & 0.93 & 0.93 & 0.89 \\
2 & 0.90 & 0.92 & 0.93 & 0.91 & 0.88 \\
3 & 0.90 & 0.92 & 0.93 & 0.91 & 0.88 \\
4 & 0.91 & 0.92 & 0.93 & 0.92 & 0.88 \\
Big & 0.90 & 0.92 & 0.94 & 0.93 & 0.89 \\
\hline
\end{tabular}

\begin{tabular}{|c|c|c|c|c|}
\hline Illiquid & 2 & 3 & 4 & Liquid \\
\hline \multicolumn{5}{|c|}{$t(a)$} \\
\hline-5.55 & -4.00 & -1.86 & 3.68 & 6.93 \\
\hline-5.63 & -3.54 & -1.16 & 4.37 & 6.71 \\
\hline-5.59 & -3.56 & -0.39 & 4.30 & 6.72 \\
\hline-5.77 & -3.48 & -0.81 & 4.31 & 7.01 \\
\hline-5.51 & -4.24 & -0.68 & 3.89 & 7.36 \\
\hline \multicolumn{5}{|c|}{$\mathrm{t}(\mathrm{b})$} \\
\hline 38.68 & 55.41 & 50.59 & 56.73 & 59.90 \\
\hline 35.44 & 49.37 & 52.13 & 56.88 & 59.91 \\
\hline 34.43 & 47.96 & 52.55 & 56.77 & 60.54 \\
\hline 34.75 & 50.93 & 52.83 & 56.40 & 60.30 \\
\hline 37.92 & 56.18 & 52.03 & 57.18 & 62.79 \\
\hline \multicolumn{5}{|c|}{$\mathrm{t}(\mathrm{s})$} \\
\hline 31.35 & 33.15 & 36.51 & 35.90 & 30.23 \\
\hline 31.88 & 33.53 & 36.09 & 33.35 & 28.83 \\
\hline 32.18 & 33.56 & 38.24 & 33.50 & 29.14 \\
\hline 32.32 & 33.12 & 38.32 & 36.20 & 30.14 \\
\hline 31.97 & 33.62 & 40.54 & 40.05 & 32.04 \\
\hline \multicolumn{5}{|c|}{$\mathrm{t}(\mathrm{h})$} \\
\hline 16.42 & 15.51 & 12.89 & 3.41 & -7.39 \\
\hline 16.77 & 15.27 & 10.63 & 1.03 & -8.05 \\
\hline 16.88 & 15.26 & 8.86 & -0.95 & -8.46 \\
\hline 17.07 & 15.20 & 9.97 & -0.66 & -8.74 \\
\hline 17.13 & 15.26 & 12.09 & 2.02 & -7.64 \\
\hline \multicolumn{5}{|c|}{$\mathrm{s}(\mathrm{e})$} \\
\hline 1.95 & 1.78 & 1.67 & 1.79 & 2.53 \\
\hline 1.90 & 1.77 & 1.69 & 2.03 & 2.75 \\
\hline 1.89 & 1.77 & 1.67 & 2.07 & 2.80 \\
\hline 1.88 & 1.78 & 1.65 & 1.91 & 2.75 \\
\hline 1.90 & 1.76 & 1.55 & 1.70 & 2.51 \\
\hline
\end{tabular}

GRS test: $F$ value $=7.34$, $p$-value $<0.0001$ 
Table 10. The Fama-French Three-Factor Model on 25 BE/ME-Corporate Liquidity Portfolios

Time series regressions of excess stock returns on the excess market returns (MKT) and the returns on size $(S M B)$ and book-tomarket equity $(H M L)$ factors: July 1963 to December 2005, 510 months.

$$
R_{t}^{i}-R F_{t}=a^{i}+b^{i} M K T_{t}+s^{i} S M B_{t}+h^{i} H M L_{t}+e_{t}
$$

Dependent variable: Excess returns on 25 portfolios subsequently sorted by BE/ME and corporate liquidity

\begin{tabular}{|c|c|c|c|c|c|c|c|c|c|c|}
\hline \multirow{3}{*}{$\begin{array}{r}\mathrm{BE} / \mathrm{ME} \\
\text { quintiles }\end{array}$} & \multicolumn{10}{|c|}{ Corporate Liquidity quintiles } \\
\hline & Illiquid & 2 & 3 & 4 & Liquid & Illiquid & 2 & 3 & 4 & Liquid \\
\hline & \multicolumn{5}{|c|}{$\mathrm{a}$} & \multicolumn{5}{|c|}{$\mathrm{t}(\mathrm{a})$} \\
\hline Low & -0.44 & -0.12 & 0.26 & 0.65 & 1.03 & -5.39 & -1.78 & 3.30 & 6.31 & 6.89 \\
\hline 2 & -0.46 & -0.24 & 0.05 & 0.47 & 0.90 & -5.56 & -3.20 & 0.69 & 5.16 & 6.93 \\
\hline 3 & -0.48 & -0.31 & -0.13 & 0.22 & 0.79 & -5.62 & -3.83 & -1.80 & 2.75 & 6.92 \\
\hline 4 & -0.49 & -0.34 & -0.16 & 0.14 & 0.71 & -5.63 & -4.14 & -2.07 & 1.82 & 6.53 \\
\hline \multirow[t]{2}{*}{ High } & -0.49 & -0.36 & -0.18 & 0.07 & 0.69 & -5.64 & -4.32 & -2.34 & 0.93 & 6.77 \\
\hline & \multicolumn{5}{|c|}{$\mathrm{b}$} & \multicolumn{5}{|c|}{$\mathrm{t}(\mathrm{b})$} \\
\hline Low & 1.03 & 1.06 & 1.09 & 1.08 & 1.08 & 27.98 & 64.28 & 55.41 & 42.19 & 54.77 \\
\hline 2 & 1.04 & 1.07 & 1.09 & 1.08 & 1.09 & 32.81 & 59.53 & 59.40 & 47.42 & 53.51 \\
\hline 3 & 1.07 & 1.09 & 1.09 & 1.10 & 1.11 & 38.26 & 55.81 & 60.64 & 54.34 & 51.89 \\
\hline 4 & 1.08 & 1.10 & 1.09 & 1.11 & 1.11 & 40.49 & 55.27 & 57.35 & 56.55 & 50.91 \\
\hline \multirow[t]{2}{*}{ High } & 1.08 & 1.11 & 1.08 & 1.10 & 1.11 & 43.36 & 54.87 & 57.07 & 60.09 & 50.32 \\
\hline & \multicolumn{5}{|c|}{$\mathrm{s}$} & \multicolumn{5}{|c|}{$\mathrm{t}(\mathrm{s})$} \\
\hline Low & 0.87 & 0.96 & 1.00 & 1.07 & 1.30 & 33.29 & 43.20 & 39.26 & 32.16 & 26.81 \\
\hline 2 & 0.87 & 0.92 & 0.98 & 1.04 & 1.23 & 32.56 & 37.91 & 40.95 & 35.05 & 29.46 \\
\hline 3 & 0.88 & 0.85 & 0.89 & 0.96 & 1.13 & 32.05 & 32.62 & 37.02 & 36.29 & 30.60 \\
\hline 4 & 0.89 & 0.83 & 0.86 & 0.89 & 1.10 & 31.59 & 31.65 & 34.03 & 34.65 & 31.10 \\
\hline \multirow[t]{2}{*}{ High } & 0.89 & 0.84 & 0.86 & 0.86 & 1.05 & 31.34 & 31.41 & 33.98 & 35.29 & 32.06 \\
\hline & \multicolumn{5}{|c|}{$\mathrm{h}$} & \multicolumn{5}{|c|}{$\mathrm{t}(\mathrm{h})$} \\
\hline Low & 0.51 & 0.32 & 0.06 & -0.21 & -0.51 & 16.89 & 12.55 & 1.90 & -5.35 & -9.16 \\
\hline 2 & 0.52 & 0.41 & 0.18 & -0.07 & -0.44 & 16.85 & 14.62 & 6.35 & -2.10 & -9.07 \\
\hline 3 & 0.54 & 0.47 & 0.34 & 0.09 & -0.32 & 17.08 & 15.35 & 12.42 & 2.79 & -7.58 \\
\hline 4 & 0.54 & 0.47 & 0.41 & 0.17 & -0.25 & 16.72 & 15.39 & 13.85 & 5.77 & -6.07 \\
\hline \multirow[t]{2}{*}{ High } & 0.54 & 0.48 & 0.42 & 0.24 & -0.21 & 16.46 & 15.52 & 14.30 & 8.37 & -5.46 \\
\hline & \multicolumn{5}{|c|}{$\mathrm{R}^{2}$} & \multicolumn{5}{|c|}{$\mathrm{s}(\mathrm{e})$} \\
\hline Low & 0.91 & 0.94 & 0.93 & 0.90 & 0.85 & 1.82 & 1.53 & 1.77 & 2.29 & 3.34 \\
\hline 2 & 0.91 & 0.93 & 0.94 & 0.91 & 0.87 & 1.85 & 1.67 & 1.65 & 2.05 & 2.89 \\
\hline 3 & 0.90 & 0.91 & 0.93 & 0.92 & 0.89 & 1.90 & 1.81 & 1.66 & 1.82 & 2.55 \\
\hline 4 & 0.90 & 0.91 & 0.92 & 0.92 & 0.90 & 1.94 & 1.82 & 1.75 & 1.77 & 2.43 \\
\hline High & 0.90 & 0.91 & 0.92 & 0.93 & 0.90 & 1.95 & 1.85 & 1.75 & 1.68 & 2.27 \\
\hline
\end{tabular}

GRS test: $F$ value $=5.59$, p-value $<0.0001$ 


\section{Table 11. The Fama-French Three-Factor + Corporate Liquidity Factor on 25 Size-Corporate Liquidity Portfolios}

Time series regressions of excess stock returns on the excess market returns $(M K T)$, the returns on size (SMB), book-tomarket equity $(H M L)$ and the corporate liquidity $(L M I)$ factors: July 1963 to December 2005, 510 months.

$$
R_{t}^{i}-R F_{t}=a^{i}+b^{i} M K T_{t}+s^{i} S M B_{t}+h^{i} H M L_{t}+c^{i} L M I_{t}+e_{t}
$$

Dependent variable: Excess returns on 25 stock portfolios formed on size and corporate liquidity

Size

\begin{tabular}{|c|c|c|c|c|c|c|c|c|c|c|}
\hline \multirow{2}{*}{$\begin{array}{r}\text { Size } \\
\text { quintiles }\end{array}$} & \\
\hline & Illiquid & 2 & 3 & 4 & Liquid & Illiquid & 2 & 3 & 4 & Liquid \\
\hline & \multicolumn{5}{|c|}{$\mathrm{a}$} & \multicolumn{5}{|c|}{$\mathrm{t}(\mathrm{a})$} \\
\hline Small & -0.04 & 0.04 & 0.03 & 0.04 & 0.03 & -0.57 & 0.57 & 0.32 & 0.48 & 0.34 \\
\hline 2 & -0.04 & 0.06 & -0.02 & -0.01 & -0.02 & -0.55 & 0.85 & -0.28 & -0.07 & -0.29 \\
\hline 3 & -0.05 & 0.06 & -0.05 & -0.08 & -0.05 & -0.66 & 0.86 & -0.66 & -0.98 & -0.54 \\
\hline 4 & -0.06 & 0.07 & 0.00 & -0.05 & -0.04 & -0.85 & 0.97 & 0.04 & -0.63 & -0.51 \\
\hline \multirow[t]{2}{*}{ Big } & -0.04 & 0.02 & 0.06 & 0.01 & 0.01 & -0.56 & 0.33 & 0.81 & 0.20 & 0.10 \\
\hline & \multicolumn{5}{|c|}{$\mathrm{b}$} & \multicolumn{5}{|c|}{$t(b)$} \\
\hline Small & 1.07 & 1.10 & 1.10 & 1.10 & 1.10 & 60.21 & 64.90 & 61.40 & 59.49 & 58.30 \\
\hline 2 & 1.08 & 1.09 & 1.11 & 1.11 & 1.10 & 62.59 & 64.21 & 59.99 & 57.06 & 55.87 \\
\hline 3 & 1.08 & 1.08 & 1.11 & 1.11 & 1.10 & 62.29 & 64.20 & 60.50 & 58.96 & 56.37 \\
\hline 4 & 1.08 & 1.07 & 1.09 & 1.08 & 1.10 & 62.73 & 63.90 & 60.37 & 61.00 & 60.45 \\
\hline \multirow[t]{2}{*}{ Big } & 1.08 & 1.07 & 1.07 & 1.06 & 1.10 & 61.73 & 65.48 & 63.43 & 61.85 & 65.38 \\
\hline & \multicolumn{5}{|c|}{$\mathrm{s}$} & \multicolumn{5}{|c|}{$\mathrm{t}(\mathrm{s})$} \\
\hline Small & 0.97 & 0.93 & 0.92 & 0.88 & 0.96 & 40.29 & 40.32 & 37.89 & 35.23 & 37.65 \\
\hline 2 & 0.96 & 0.93 & 0.90 & 0.90 & 0.98 & 41.34 & 40.06 & 35.73 & 34.12 & 36.96 \\
\hline 3 & 0.96 & 0.93 & 0.92 & 0.91 & 1.01 & 41.04 & 40.18 & 36.96 & 35.88 & 38.68 \\
\hline 4 & 0.96 & 0.92 & 0.93 & 0.92 & 1.02 & 41.29 & 39.79 & 37.89 & 38.31 & 42.47 \\
\hline \multirow[t]{2}{*}{ Big } & 0.96 & 0.93 & 0.93 & 0.93 & 1.00 & 40.84 & 40.88 & 40.87 & 40.19 & 45.32 \\
\hline & \multicolumn{5}{|c|}{$\mathrm{h}$} & \multicolumn{5}{|c|}{$\mathrm{t}(\mathrm{h})$} \\
\hline Small & 0.24 & 0.22 & 0.25 & 0.27 & 0.19 & 7.36 & 7.05 & 7.59 & 7.95 & 5.42 \\
\hline 2 & 0.24 & 0.22 & 0.26 & 0.30 & 0.19 & 7.60 & 7.05 & 7.47 & 8.35 & 5.32 \\
\hline 3 & 0.25 & 0.22 & 0.26 & 0.29 & 0.19 & 7.87 & 7.02 & 7.71 & 8.19 & 5.39 \\
\hline 4 & 0.26 & 0.22 & 0.23 & 0.26 & 0.20 & 8.04 & 6.92 & 6.95 & 7.78 & 5.94 \\
\hline Big & 0.26 & 0.21 & 0.24 & 0.24 & 0.22 & 8.10 & 6.80 & 7.78 & 7.68 & 7.37 \\
\hline
\end{tabular}

Corporate Liquidity quintiles 
Table 11. (continued)

\begin{tabular}{|c|c|c|c|c|c|c|c|c|c|c|}
\hline \multirow[b]{2}{*}{ Small } & \multicolumn{5}{|c|}{$\mathrm{c}$} & \multicolumn{5}{|c|}{$\mathrm{t}(\mathrm{c})$} \\
\hline & -0.43 & -0.35 & -0.16 & 0.25 & 0.73 & -15.36 & -13.25 & -5.73 & 8.65 & 25.06 \\
\hline 2 & -0.42 & -0.33 & -0.06 & 0.39 & 0.82 & -15.80 & -12.51 & -2.20 & 12.85 & 27.02 \\
\hline 3 & -0.41 & -0.34 & 0.02 & 0.46 & 0.86 & -15.14 & -12.61 & 0.80 & 15.90 & 28.74 \\
\hline 4 & -0.41 & -0.34 & -0.06 & 0.41 & 0.87 & -15.23 & -12.72 & -2.16 & 14.67 & 31.60 \\
\hline \multirow[t]{2}{*}{ Big } & -0.41 & -0.35 & -0.10 & 0.27 & 0.80 & -15.20 & -13.29 & -3.94 & 10.23 & 31.25 \\
\hline & \multicolumn{5}{|c|}{$\mathrm{R}^{2}$} & \multicolumn{5}{|c|}{$s(e)$} \\
\hline Small & 0.93 & 0.94 & 0.93 & 0.93 & 0.95 & 1.62 & 1.54 & 1.62 & 1.68 & 1.71 \\
\hline 2 & 0.93 & 0.94 & 0.93 & 0.93 & 0.95 & 1.56 & 1.55 & 1.68 & 1.77 & 1.78 \\
\hline 3 & 0.93 & 0.94 & 0.93 & 0.94 & 0.95 & 1.58 & 1.55 & 1.67 & 1.70 & 1.75 \\
\hline 4 & 0.93 & 0.94 & 0.93 & 0.94 & 0.96 & 1.56 & 1.56 & 1.65 & 1.61 & 1.61 \\
\hline Big & 0.93 & 0.94 & 0.94 & 0.94 & 0.96 & 1.58 & 1.53 & 1.53 & 1.55 & 1.48 \\
\hline
\end{tabular}

GRS test: $F$ value $=4.21, p$-value $<0.0001$ 


\section{Table 12. The Fama-French Three-Factor + Corporate Liquidity Factor on 25 BE/ME-Corporate Liquidity Portfolios}

Time series regressions of excess stock returns on the excess market returns $(M K T)$, the returns on size (SMB), book-to-market equity $(H M L)$ and the corporate liquidity $(L M I)$ factors: July 1963 to December 2005, 510 months.

$$
R_{t}^{i}-R F_{t}=a^{i}+b^{i} M K T_{t}+s^{i} S M B_{t}+h^{i} H M L_{t}+c^{i} L M I_{t}+e_{t}
$$

Dependent variable: Excess returns on 25 stock portfolios formed on book-to-market equity and corporate liquidity

\begin{tabular}{|c|c|c|c|c|c|c|c|c|c|c|}
\hline \multirow{3}{*}{$\begin{array}{c}\mathrm{BE} / \mathrm{ME} \\
\text { quintiles }\end{array}$} & \multicolumn{10}{|c|}{ Corporate Liquidity quintiles } \\
\hline & Illiquid & 2 & 3 & 4 & Liquid & Illiquid & 2 & 3 & 4 & Liquid \\
\hline & \multicolumn{5}{|c|}{$\mathrm{a}$} & \multicolumn{5}{|c|}{$\mathrm{t}(\mathrm{a})$} \\
\hline Low & -0.04 & -0.01 & -0.06 & 0.00 & -0.05 & -0.49 & -0.18 & -0.83 & -0.01 & -0.47 \\
\hline 2 & -0.05 & 0.03 & -0.08 & -0.04 & -0.06 & -0.66 & 0.34 & -1.05 & -0.55 & -0.70 \\
\hline 3 & -0.05 & 0.06 & 0.02 & -0.06 & 0.00 & -0.60 & 0.77 & 0.24 & -0.77 & -0.05 \\
\hline 4 & -0.04 & 0.04 & 0.09 & 0.00 & 0.00 & -0.58 & 0.54 & 1.14 & 0.01 & -0.03 \\
\hline \multirow[t]{2}{*}{ High } & -0.05 & 0.03 & 0.10 & 0.04 & 0.05 & -0.65 & 0.37 & 1.33 & 0.53 & 0.64 \\
\hline & \multicolumn{5}{|c|}{$\mathrm{b}$} & \multicolumn{5}{|c|}{$t(b)$} \\
\hline Low & 1.08 & 1.08 & 1.08 & 1.06 & 1.08 & 64.69 & 65.02 & 62.49 & 59.77 & 47.24 \\
\hline 2 & 1.08 & 1.09 & 1.08 & 1.07 & 1.08 & 63.31 & 64.17 & 60.69 & 61.15 & 57.67 \\
\hline 3 & 1.08 & 1.10 & 1.10 & 1.09 & 1.09 & 61.97 & 63.90 & 61.94 & 59.24 & 60.91 \\
\hline 4 & 1.08 & 1.10 & 1.10 & 1.10 & 1.11 & 60.95 & 63.66 & 60.89 & 57.81 & 59.63 \\
\hline \multirow[t]{2}{*}{ High } & 1.07 & 1.10 & 1.09 & 1.10 & 1.11 & 59.98 & 63.34 & 61.84 & 60.06 & 60.98 \\
\hline & \multicolumn{5}{|c|}{$\mathrm{S}$} & \multicolumn{5}{|c|}{$\mathrm{t}(\mathrm{s})$} \\
\hline Low & 0.95 & 0.98 & 0.94 & 0.94 & 1.08 & 42.12 & 43.58 & 40.01 & 38.39 & 35.84 \\
\hline 2 & 0.96 & 0.97 & 0.95 & 0.94 & 1.04 & 41.38 & 42.28 & 39.48 & 39.16 & 41.68 \\
\hline 3 & 0.97 & 0.93 & 0.92 & 0.90 & 0.97 & 41.26 & 39.78 & 38.16 & 35.97 & 40.03 \\
\hline 4 & 0.98 & 0.91 & 0.91 & 0.86 & 0.95 & 40.87 & 38.98 & 37.40 & 33.24 & 38.15 \\
\hline High & 0.97 & 0.92 & 0.92 & 0.85 & 0.93 & 40.37 & 38.82 & 38.42 & 34.08 & 38.03 \\
\hline & \multicolumn{5}{|c|}{$\mathrm{h}$} & \multicolumn{5}{|c|}{$\mathrm{t}(\mathrm{h})$} \\
\hline Low & 0.25 & 0.25 & 0.27 & 0.23 & 0.20 & 7.93 & 8.07 & 8.40 & 6.70 & 4.85 \\
\hline 2 & 0.25 & 0.23 & 0.26 & 0.27 & 0.19 & 7.86 & 7.42 & 7.96 & 8.22 & 5.61 \\
\hline 3 & 0.26 & 0.22 & 0.24 & 0.27 & 0.20 & 7.97 & 6.89 & 7.37 & 8.00 & 6.11 \\
\hline 4 & 0.25 & 0.22 & 0.24 & 0.27 & 0.23 & 7.58 & 6.83 & 7.13 & 7.50 & 6.60 \\
\hline High & 0.24 & 0.22 & 0.23 & 0.25 & 0.22 & 7.38 & 6.92 & 7.00 & 7.36 & 6.44 \\
\hline
\end{tabular}


Table 12. (continued)

\begin{tabular}{|c|c|c|c|c|c|c|c|c|c|c|}
\hline \multirow[b]{2}{*}{ Low } & \multicolumn{5}{|c|}{$\mathrm{c}$} & \multicolumn{5}{|c|}{$\mathrm{t}(\mathrm{c})$} \\
\hline & -0.39 & -0.11 & 0.31 & 0.63 & 1.04 & -14.94 & -4.11 & 11.60 & 22.33 & 29.95 \\
\hline 2 & -0.40 & -0.26 & 0.13 & 0.50 & 0.92 & -15.03 & -9.72 & 4.63 & 18.13 & 32.03 \\
\hline 3 & -0.42 & -0.36 & -0.15 & 0.28 & 0.77 & -15.53 & -13.30 & -5.32 & 9.62 & 27.55 \\
\hline 4 & -0.43 & -0.37 & -0.24 & 0.14 & 0.69 & -15.66 & -13.63 & -8.69 & 4.66 & 24.04 \\
\hline \multirow[t]{2}{*}{ High } & -0.43 & -0.37 & -0.28 & 0.03 & 0.62 & -15.45 & -13.74 & -10.07 & 0.89 & 22.02 \\
\hline & \multicolumn{5}{|c|}{$\mathrm{R}^{2}$} & \multicolumn{5}{|c|}{$\mathrm{s}(\mathrm{e})$} \\
\hline Low & 0.94 & 0.94 & 0.94 & 0.95 & 0.94 & 1.52 & 1.50 & 1.58 & 1.64 & 2.03 \\
\hline 2 & 0.94 & 0.94 & 0.94 & 0.95 & 0.96 & 1.55 & 1.54 & 1.62 & 1.61 & 1.68 \\
\hline 3 & 0.93 & 0.94 & 0.93 & 0.94 & 0.96 & 1.58 & 1.57 & 1.61 & 1.68 & 1.63 \\
\hline 4 & 0.93 & 0.93 & 0.93 & 0.93 & 0.95 & 1.60 & 1.57 & 1.64 & 1.74 & 1.68 \\
\hline High & 0.93 & 0.93 & 0.93 & 0.93 & 0.95 & 1.62 & 1.58 & 1.61 & 1.68 & 1.63 \\
\hline
\end{tabular}

GRS test: $F$ value $=2.48, p$-value $=0.0001$ 


\section{Table 13. Time Series Regression of Corporate Liquidity Factor on Changes in Macroeconomic Variables}

Time series regression of corporate liquidity on changes in macroeconomic variables. The dependent variable $L M I$ is the aggregate corporate liquidity factor. Independent variables are current and two forward values of macroeconomic variables: $\triangle G D P, \triangle I P, \triangle C P I, \triangle F F R$, or NBER recession dummy. The time series regression is performed during the period 1962:2005.

$$
\text { LMI }_{t}=\alpha+\sum_{j=0}^{2} \delta_{j} \Delta \text { MacroCondition }_{t+j}+\varepsilon_{t}
$$

\begin{tabular}{|c|c|c|c|c|c|}
\hline & (1) & (2) & $(3)$ & (4) & $(5)$ \\
\hline \multirow[t]{2}{*}{$\Delta G D P_{t}$} & -1.044 & - & - & - & - \\
\hline & $(-1.312)$ & - & - & - & - \\
\hline \multirow[t]{2}{*}{$\Delta G D P_{t+1}$} & 1.567 & - & - & - & - \\
\hline & $(1.487)$ & - & - & - & - \\
\hline \multirow[t]{2}{*}{$\Delta G D P_{t+2}$} & -0.819 & - & - & - & - \\
\hline & $(-1.029)$ & - & - & - & - \\
\hline \multirow[t]{2}{*}{$\Delta$ Industrial Production $_{t}$} & - & -0.142 & - & - & - \\
\hline & - & $(-0.650)$ & - & - & - \\
\hline \multirow[t]{2}{*}{$\Delta$ Industrial Production $_{t+1}$} & - & 0.027 & - & - & - \\
\hline & - & $(0.120)$ & - & - & - \\
\hline \multirow[t]{2}{*}{$\Delta$ Industrial Production $_{t+2}$} & - & 0.148 & - & - & - \\
\hline & - & $(0.679)$ & - & - & - \\
\hline \multirow[t]{2}{*}{$\triangle C P I_{t}$} & - & - & 0.151 & - & - \\
\hline & - & - & $(0.275)$ & - & - \\
\hline \multirow[t]{2}{*}{$\Delta C P I_{t+1}$} & - & - & -1.168 & - & - \\
\hline & - & - & $(-1.990)^{* *}$ & - & - \\
\hline \multirow[t]{2}{*}{$\triangle C P I_{t+2}$} & - & - & 0.943 & - & - \\
\hline & - & - & $(1.736)^{*}$ & - & - \\
\hline \multirow[t]{2}{*}{$\Delta F F R_{t}$} & - & - & - & -0.221 & - \\
\hline & - & - & - & $(-0.793)$ & - \\
\hline \multirow{2}{*}{$\Delta F F R_{t+1}$} & - & - & - & 0.103 & - \\
\hline & - & - & - & $(0.340)$ & - \\
\hline \multirow[t]{2}{*}{$\Delta F F R_{t+2}$} & - & - & - & 0.012 & - \\
\hline & - & - & - & $(0.044)$ & - \\
\hline \multirow[t]{2}{*}{$\Delta$ Recession $_{t}$} & - & - & - & - & -0.077 \\
\hline & - & - & - & - & $(-0.076)$ \\
\hline \multirow[t]{2}{*}{$\Delta$ Recession $_{t+1}$} & - & - & - & - & 0.713 \\
\hline & - & - & - & - & $(0.516)$ \\
\hline \multirow[t]{2}{*}{$\Delta$ Recession $_{t+2}$} & - & - & - & - & -0.592 \\
\hline & - & - & - & - & $(-0.591)$ \\
\hline$R^{2}$ & 0.056 & 0.061 & 0.072 & 0.045 & 0.047 \\
\hline
\end{tabular}




\section{Table 14. Performance on Macroeconomic Changes for Liquid and Illiquid Firms}

Time series regression of firm performance on macroeconomic changes for liquid and illiquid firms during the period 1962:2005. Every year all firms are sorted into liquid and illiquid portfolios where liquid firms are defined as firms whose cash holdings fall into the top 30 percentile, while illiquid firms are those with the bottom 30 percentile cash holdings. Performance is measured by median growth rate of earnings before interest and taxes (EBIT, COMPUSTAT item 14 + item 18), sales (item 12) and investment (item 128). MacroCondition include (1) aggregate demand, proxied by GDP - gross domestic production; (2) aggregate production, proxied by $I P$ industrial production index; (3) an NBER recession dummy variable, and (4) federal funds rate (FFR), a proxy for monetary policy. Federal funds rate is included in the bivariate regression with GDP to control for the monetary policy changes that can coincide with macroeconomic changes. In addition, the recession dummy variable takes a value of -1 for the year under recession in order to conveniently compare its regression coefficient with other macroeconomic changes.

$$
\text { Performance }_{i, t}=\alpha+\delta \Delta \text { MacroCondition }_{t}+\phi \Delta F F R_{t}+\rho \text { Trend }_{t}+\varepsilon_{i, t}
$$

Macroeconomic Variables

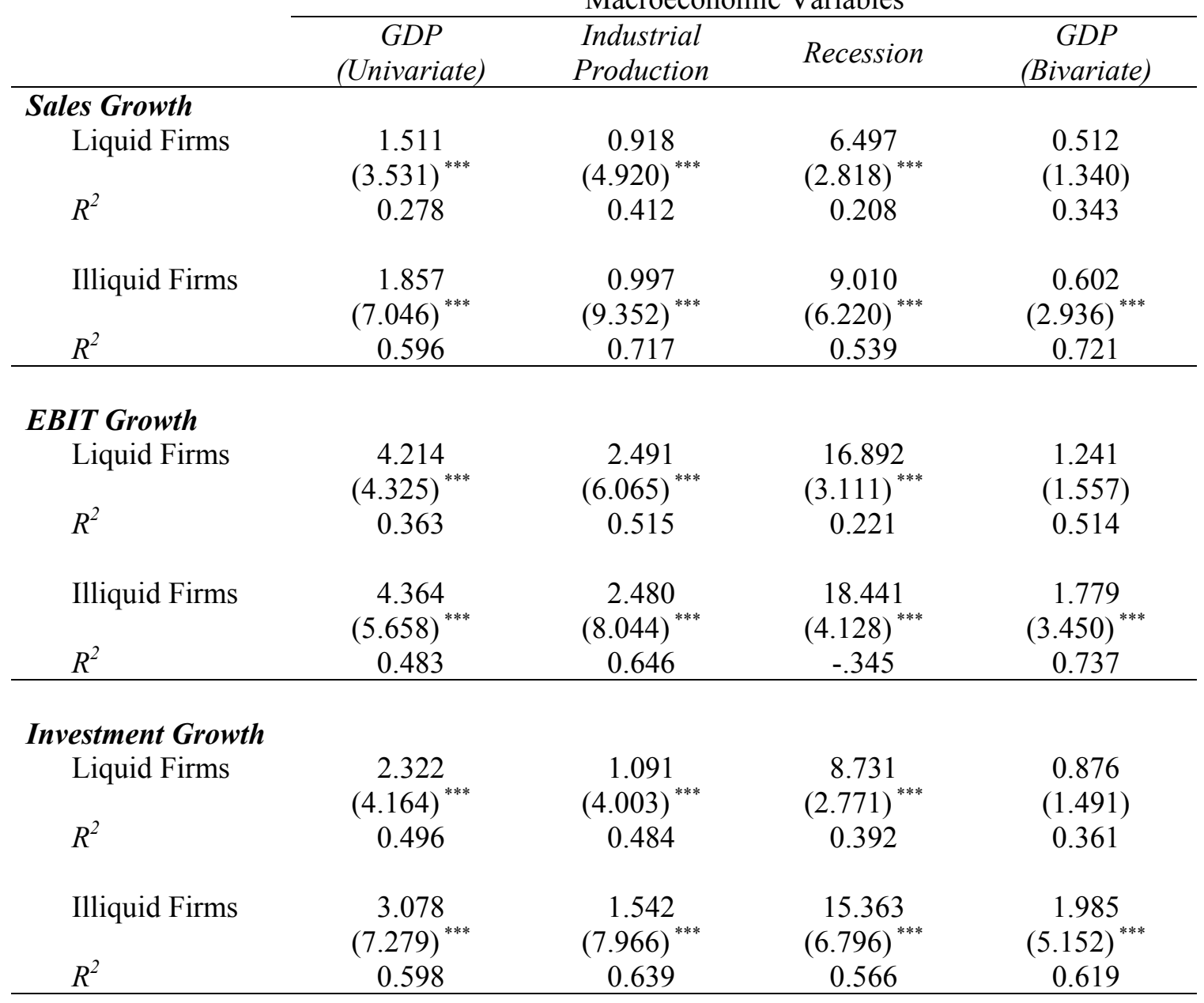


Table 15. Time Series Regression of Corporate Liquidity Beta on Changes in Macroeconomic Conditions for Liquid and Illiquid Firms

This table presents the result of the second step, a time series regression, of a two-step regression approach for the period 1962:2005. In the first step, time series regressions are estimated for liquidity beta on a rolling basis for the liquid and illiquid portfolio separately every month using the previous 36 months data points, in which portfolios' excess returns are regressed on the factors $L M I, M K T, S M B$ and $H M L$. The coefficients on the corporate liquidity factor $L M I$ are collected and "stacked" into the vector $\theta_{t}$, which is then regressed on changes in the various macroeconomic variables, including current and two lagged $\triangle G D P$, contemporaneous $\log C P I$, changes in the federal funds rate $\log F F R_{2}$ and a time trend.

$$
\theta_{t}=\alpha+\sum_{k=0}^{2} \delta_{k} \Delta G D P_{t-k}+\varphi \log C P I_{t}+\lambda \operatorname{LogFFR}+\rho \operatorname{Trend}_{t}+\mu_{t}
$$

\begin{tabular}{lllllll} 
& \multicolumn{2}{c}{$(1)$} & $(2)$ & & $(3)$ \\
\cline { 2 - 7 }$\Delta G D P_{t}$ & Liquid & Illiquid & Liquid & Illiquid & Liquid & Illiquid \\
\cline { 2 - 7 } & -1.023 & -0.995 & -11.658 & -10.473 & -8.840 & -8.012 \\
\multirow{4}{*}{$\Delta G P_{t-1}$} & $(-16.682)^{* * *}$ & $(-18.347)^{* * *}$ & $(-3.980)^{* * *}$ & $(-4.269)^{* * *}$ & $(-2.346)^{* * *}$ & $(-2.044)^{* *}$ \\
& - & - & 10.137 & 9.699 & 4.441 & 3.356 \\
$\Delta G D P_{t-2}$ & - & - & $(3.378)^{* * *}$ & $(3.522)^{* * *}$ & $(0.627)$ & $(0.437)$ \\
& - & - & - & - & 3.660 & 2.801 \\
& - & - & - & - & $(-0.868)$ & $(0.720)$ \\
\hline
\end{tabular}

$\begin{array}{lllllll}R^{2} & 0.704 & 0.695 & 0.721 & 0.699 & 0.737 & 0.714\end{array}$


Table 16. Time Series Regression of Corporate Liquidity Beta on Changes in Macroeconomic Conditions for Constrained and Unconstrained Firms

This table presents the results of the second step time series regression of a two-step regression approach for the period 1962:2005. Each year, the sample is split into constrained and unconstrained firms based on the following criteria: firm size, payout ratio and a KZ index. Constrained firms are defined as those who are ranked below the bottom 30 percentile according to firm size, payout ratio, and above the top 30 percentile KZ index; unconstrained firms are defined as those who are ranked above the top 30 percentile according to firm size, payout ratio, and below the bottom 30 percentile KZ index. In the first step, a cross sectional regression is estimated across the firms that belong to the constrained and unconstrained portfolio separately for each year, in which firms' or portfolios' excess returns are regressed on the corporate liquidity, firm size, market to book, leverage and other firm characteristics. The coefficients on corporate liquidity are collected and "stacked" into the vector $\theta_{t}$, which is then regressed on various variables that describe macroeconomic changes, including current and two lagged GDP changes, and contemporaneous $\log C P I$, changes in federal funds rate $\log F F R$ and a time trend.

$$
\theta_{t}=\alpha+\delta \Delta G D P_{t}+\varphi \operatorname{LogCPI}_{t}+\lambda \operatorname{LogFFR}_{t}+\rho \text { Trend }_{t}+\mu_{t}
$$

1. Total Assets

(1)

(2)

\begin{tabular}{|c|c|c|c|c|c|c|}
\hline & Constrained & Unconstrained & Constrained & Unconstrained & Constrained & Unconstrained \\
\hline GDPInnovation $_{t}$ & $\begin{array}{c}-0.129 \\
(-2.404)^{* *}\end{array}$ & $\begin{array}{c}-0.014 \\
(-0.273)\end{array}$ & $\begin{array}{c}-0.120 \\
(-2.617)^{* * *}\end{array}$ & $\begin{array}{c}0.023 \\
(0.320)\end{array}$ & $\begin{array}{c}-0.110 \\
(-2.545)^{* *}\end{array}$ & $\begin{array}{c}0.024 \\
(0.253)\end{array}$ \\
\hline GDPInnovation $_{t-1}$ & - & $\begin{array}{l}- \\
-\end{array}$ & $\begin{array}{c}-0.111 \\
(-2.431)^{* *}\end{array}$ & $\begin{array}{c}0.046 \\
(0.320)\end{array}$ & $\begin{array}{c}-0.104 \\
(-2.373)^{* *}\end{array}$ & $\begin{array}{c}0.078 \\
(0.871)\end{array}$ \\
\hline GDPInnovation $_{t-2}$ & - & - & - & $\begin{array}{l}- \\
-\end{array}$ & $\begin{array}{c}0.040 \\
(0.938) \\
\end{array}$ & $\begin{array}{c}-0.014 \\
(-0.197)\end{array}$ \\
\hline$R^{2}$ & 0.159 & 0.050 & 0.413 & 0.067 & 0.419 & 0.082 \\
\hline
\end{tabular}


Table 16. (continued)

2. Payout ratio

\begin{tabular}{lcc|cc|cc} 
& \multicolumn{3}{c}{$(1)$} & \multicolumn{2}{c}{$(3)$} \\
\cline { 2 - 7 } GDPInnovation $_{t}$ & Constrained & Unconstrained & Constrained & Unconstrained & Constrained & Unconstrained \\
\cline { 2 - 7 } & -0.082 & -0.007 & -0.162 & 0.001 & -0.111 & -0.038 \\
GDPInnovation $_{t-1}$ & $(-1.334)$ & $(-0.232)$ & $(-1.880)^{*}$ & $(0.019)$ & $(-1.244)$ & $(-0.718)$ \\
& - & - & -0.029 & 0.037 & -0.037 & 0.046 \\
GDPInnovation $_{t-2}$ & - & - & $-0.395)$ & $(1.044)$ & $(-0.435)$ & $(0.933)$ \\
& - & - & - & - & 0.063 & -0.002 \\
$R^{2}$ & 0.155 & 0.061 & 0.201 & 0.093 & 0.144 & 0.108 \\
\hline
\end{tabular}

3. KZ index

(1)

(2)

(3)

\begin{tabular}{lcc|cc|cc} 
& Constrained & Unconstrained & Constrained & Unconstrained & Constrained & Unconstrained \\
\cline { 2 - 7 } GDPInnovation $_{t}$ & -0.090 & -0.006 & -0.169 & 0.002 & -0.104 & -0.036 \\
& $(-1.378)$ & $(-0.174)$ & $(-1.866)^{*}$ & $(0.052)$ & $(-1.088)$ & $(-0.593)$ \\
GDPInnovation $_{t-1}$ & - & - & -0.030 & 0.031 & -0.055 & 0.036 \\
& - & - & $(-0.388)$ & $(0.767)$ & $(-0.612)$ & $(0.633)$ \\
GDPInnovation $_{t-2}$ & - & - & - & - & 0.041 & -0.007 \\
& - & - & - & - & $(0.601)$ & $(-0.002)$ \\
\hline \multirow{2}{*}{$R^{2}$} & 0.154 & 0.057 & 0.207 & 0.088 & 0.167 & 0.120 \\
\hline
\end{tabular}




\section{Table A1. The Fama-French Three-Factor + Corporate Liquidity Factor $\left(L M I_{\text {med_low }}\right)$} on 27 Size-BE/ME-Corporate Liquidity Portfolios

Time series regressions of excess stock returns on the excess market returns $(M K T)$, the returns on size $(S M B)$, book-to-market equity $(H M L)$ and the corporate liquidity $\left(L M I_{\text {med low }}\right)$ factors: July 1963 to December 2005, 510 months.

$$
R_{t}^{i}-R F_{t}=a^{i}+b^{i} M K T_{t}+s^{i} S M B_{t}+h^{i} H M L_{t}+c^{i} L M I_{\text {med_low }}+e_{t}
$$

Dependent variable: Excess returns on 27 stock portfolios formed on size, book-to-market equity and corporate liquidity.

Corporate Liquidity Tertiles - Illiquid

\begin{tabular}{|c|c|c|c|c|c|c|}
\hline \multirow{3}{*}{ Size Tertiles } & \multicolumn{6}{|c|}{ Book-to-market Equity (BE/ME) Tertiles } \\
\hline & Low & Medium & High & Low & Medium & High \\
\hline & \multicolumn{3}{|c|}{$\mathrm{a}$} & \multicolumn{3}{|c|}{$\mathrm{t}(\mathrm{a})$} \\
\hline Small & 0.12 & 0.13 & -0.25 & 0.32 & 0.35 & -1.26 \\
\hline Medium & -0.44 & -0.19 & -0.14 & -2.04 & -0.45 & -0.13 \\
\hline \multirow[t]{2}{*}{ Big } & 0.39 & 0.26 & 0.05 & 1.95 & 0.77 & 0.87 \\
\hline & \multicolumn{3}{|c|}{$\mathrm{b}$} & \multicolumn{3}{|c|}{$\mathrm{t}(\mathrm{b})$} \\
\hline Small & 1.27 & 1.01 & 0.99 & 13.00 & 15.22 & 22.41 \\
\hline Medium & 1.25 & 1.08 & 1.04 & 20.76 & 29.08 & 32.71 \\
\hline \multirow[t]{2}{*}{ Big } & 1.11 & 1.10 & 1.16 & 30.34 & 35.81 & 30.57 \\
\hline & \multicolumn{3}{|c|}{$\mathrm{S}$} & \multicolumn{3}{|c|}{$\mathrm{t}(\mathrm{s})$} \\
\hline Small & 1.50 & 1.17 & 1.26 & 11.54 & 13.31 & 21.55 \\
\hline Medium & 0.92 & 0.92 & 0.80 & 11.55 & 18.58 & 18.96 \\
\hline \multirow[t]{2}{*}{ Big } & 0.31 & 0.34 & 0.39 & 6.29 & 8.50 & 7.78 \\
\hline & \multicolumn{3}{|c|}{$\mathrm{h}$} & \multicolumn{3}{|c|}{$\mathrm{t}(\mathrm{h})$} \\
\hline Small & 0.64 & 0.43 & 0.66 & 3.77 & 3.78 & 8.64 \\
\hline Medium & 0.16 & 0.36 & 0.55 & 1.55 & 5.53 & 9.91 \\
\hline \multirow[t]{2}{*}{ Big } & -0.02 & 0.33 & 0.51 & -0.28 & 6.24 & 7.74 \\
\hline & \multicolumn{3}{|c|}{$\mathrm{c}$} & \multicolumn{3}{|c|}{$t(c)$} \\
\hline Small & -1.19 & -0.45 & -0.90 & -3.37 & -1.87 & -5.64 \\
\hline Medium & -1.35 & -0.92 & -0.81 & -6.21 & -6.85 & -7.05 \\
\hline \multirow[t]{2}{*}{ Big } & -1.19 & -0.82 & -0.85 & -8.98 & -7.44 & -6.15 \\
\hline & \multicolumn{3}{|c|}{$\mathrm{R} 2$} & \multicolumn{3}{|c|}{$\mathrm{s}(\mathrm{e})$} \\
\hline Small & 0.74 & 0.75 & 0.79 & 4.41 & 3.23 & 3.22 \\
\hline Medium & 0.76 & 0.69 & 0.68 & 3.75 & 3.98 & 3.43 \\
\hline Big & 0.81 & 0.82 & 0.62 & 2.54 & 2.61 & 3.74 \\
\hline
\end{tabular}


Table A1. (continued)

Corporate Liquidity Tertiles - Medium

\begin{tabular}{|c|c|c|c|c|c|c|}
\hline \multirow{3}{*}{ Size Tertiles } & \multicolumn{6}{|c|}{ Book-to-market Equity (BE/ME) Tertiles } \\
\hline & Low & Medium & High & Low & Medium & High \\
\hline & \multicolumn{3}{|c|}{$\mathrm{a}$} & \multicolumn{3}{|c|}{$\mathrm{t}(\mathrm{a})$} \\
\hline Small & -0.39 & 0.22 & 0.23 & -1.06 & 1.13 & 1.31 \\
\hline Medium & 0.43 & -0.19 & -0.22 & 1.81 & -0.67 & -1.32 \\
\hline \multirow[t]{2}{*}{ Big } & -0.43 & 0.23 & 0.10 & 1.86 & 0.54 & 0.46 \\
\hline & \multicolumn{3}{|c|}{$\mathrm{b}$} & \multicolumn{3}{|c|}{$\mathrm{t}(\mathrm{b})$} \\
\hline Small & 0.97 & 0.91 & 1.02 & 10.70 & 11.75 & 19.45 \\
\hline Medium & 1.12 & 1.10 & 1.12 & 15.43 & 26.70 & 28.83 \\
\hline \multirow[t]{2}{*}{ Big } & 1.02 & 1.07 & 1.07 & 27.94 & 32.71 & 25.08 \\
\hline & \multicolumn{3}{|c|}{$\mathrm{s}$} & \multicolumn{3}{|c|}{$\mathrm{t}(\mathrm{s})$} \\
\hline Small & 1.59 & 1.23 & 1.17 & 13.23 & 12.02 & 16.79 \\
\hline Medium & 0.97 & 0.81 & 0.90 & 10.11 & 14.88 & 17.50 \\
\hline \multirow[t]{2}{*}{ Big } & 0.28 & 0.29 & 0.41 & 5.69 & 6.69 & 7.29 \\
\hline & \multicolumn{3}{|c|}{$\mathrm{h}$} & \multicolumn{3}{|c|}{$\mathrm{t}(\mathrm{h})$} \\
\hline Small & 0.63 & 0.51 & 0.66 & 4.03 & 3.78 & 7.31 \\
\hline Medium & 0.30 & 0.33 & 0.59 & 2.41 & 4.63 & 8.71 \\
\hline \multirow[t]{2}{*}{ Big } & -0.05 & 0.35 & 0.56 & -0.84 & 6.16 & 7.50 \\
\hline & \multicolumn{3}{|c|}{$\mathrm{c}$} & \multicolumn{3}{|c|}{$t(c)$} \\
\hline Small & 0.57 & -0.14 & 0.24 & 0.88 & -0.34 & 1.09 \\
\hline Medium & -0.15 & -0.16 & -0.34 & -0.29 & -0.45 & -0.98 \\
\hline \multirow[t]{2}{*}{ Big } & -0.12 & -0.32 & 0.45 & -0.39 & -0.43 & 0.63 \\
\hline & \multicolumn{3}{|c|}{$\mathrm{R} 2$} & \multicolumn{3}{|c|}{$\mathrm{s}(\mathrm{e})$} \\
\hline Small & 0.72 & 0.70 & 0.76 & 5.29 & 4.34 & 3.09 \\
\hline Medium & 0.77 & 0.68 & 0.64 & 4.15 & 4.56 & 3.98 \\
\hline Big & 0.82 & 0.72 & 0.74 & 2.25 & 3.88 & 3.34 \\
\hline
\end{tabular}


Table A1. (continued)

Corporate Liquidity Tertiles - Liquid

Book-to-market Equity (BE/ME) Tertiles

\begin{tabular}{|c|c|c|c|c|c|c|}
\hline \multirow[t]{2}{*}{ Size Tertiles } & Low & Medium & High & Low & Medium & High \\
\hline & \multicolumn{3}{|c|}{$\mathrm{a}$} & \multicolumn{3}{|c|}{$\mathrm{t}(\mathrm{a})$} \\
\hline Small & 0.22 & 0.33 & 0.49 & 0.45 & 1.09 & 1.97 \\
\hline Medium & -0.06 & 0.28 & 0.38 & -0.11 & 0.98 & 1.59 \\
\hline \multirow[t]{2}{*}{ Big } & 0.90 & 0.34 & 0.05 & 3.45 & 1.89 & 0.76 \\
\hline & \multicolumn{3}{|c|}{$b$} & \multicolumn{3}{|c|}{$t(b)$} \\
\hline Small & 1.16 & 1.10 & 0.94 & 11.27 & 13.59 & 16.03 \\
\hline Medium & 1.18 & 1.11 & 0.92 & 16.20 & 21.79 & 16.44 \\
\hline \multirow[t]{2}{*}{ Big } & 0.89 & 1.07 & 0.93 & 13.49 & 18.21 & 19.51 \\
\hline & \multicolumn{3}{|c|}{$\mathrm{S}$} & \multicolumn{3}{|c|}{$\mathrm{t}(\mathrm{s})$} \\
\hline Small & 1.17 & 0.97 & 1.06 & 8.56 & 9.03 & 13.55 \\
\hline Medium & 0.98 & 0.77 & 0.86 & 10.15 & 11.35 & 11.67 \\
\hline \multirow[t]{2}{*}{ Big } & 0.67 & 0.34 & 0.31 & 7.72 & 4.35 & 4.84 \\
\hline & \multicolumn{3}{|c|}{$\mathrm{h}$} & \multicolumn{3}{|c|}{$t(h)$} \\
\hline Small & 0.37 & 0.54 & 0.66 & 2.05 & 3.83 & 6.41 \\
\hline Medium & 0.16 & 0.31 & 0.54 & 1.30 & 3.54 & 5.54 \\
\hline \multirow[t]{2}{*}{ Big } & -0.05 & 0.20 & 0.34 & -0.47 & 1.99 & 4.09 \\
\hline & \multicolumn{3}{|c|}{$\mathrm{c}$} & \multicolumn{3}{|c|}{$t(c)$} \\
\hline Small & -0.57 & 0.49 & 0.56 & -0.94 & 0.85 & 1.36 \\
\hline Medium & -0.15 & -0.38 & -0.62 & -0.23 & -0.69 & -1.29 \\
\hline \multirow[t]{2}{*}{ Big } & 0.29 & 0.12 & 0.98 & 0.66 & 0.23 & 1.83 \\
\hline & \multicolumn{3}{|c|}{$\mathrm{R} 2$} & \multicolumn{3}{|c|}{$\mathrm{s}(\mathrm{e})$} \\
\hline Small & 0.82 & 0.79 & 0.75 & 4.06 & 4.77 & 3.98 \\
\hline Medium & 0.79 & 0.69 & 0.68 & 4.59 & 4.99 & 5.01 \\
\hline Big & 0.82 & 0.77 & 0.65 & 3.87 & 4.86 & 5.10 \\
\hline
\end{tabular}

GRS test: $F$ value $=1.38, p$-value $=0.11$ 
Table A2. The Fama-French Three-Factor + Corporate Liquidity Factor $\left(\boldsymbol{L M I}_{\text {high_med }}\right)$ on 27 Size-BE/ME-Corporate Liquidity Portfolios

Time series regressions of excess stock returns on the excess market returns $(M K T)$, the returns on size $(S M B)$, book-to-market equity $(H M L)$ and the corporate liquidity $\left(L M I_{\text {high_med }}\right)$ factors: July 1963 to December 2005 , 510 months.

$$
R_{t}^{i}-R F_{t}=a^{i}+b^{i} M K T_{t}+s^{i} S M B_{t}+h^{i} H M L_{t}+c^{i} L M I_{\text {high }_{-} \text {med }_{t}}+e_{t}
$$

Dependent variable: Excess returns on 27 stock portfolios formed on size, book-to-market equity and corporate liquidity.

Corporate Liquidity Tertiles - Illiquid

\begin{tabular}{|c|c|c|c|c|c|c|}
\hline \multirow{3}{*}{ Size Tertiles } & \multicolumn{6}{|c|}{ Book-to-market Equity (BE/ME) Tertiles } \\
\hline & Low & Medium & High & Low & Medium & High \\
\hline & \multicolumn{3}{|c|}{$\mathrm{a}$} & \multicolumn{3}{|c|}{$\mathrm{t}(\mathrm{a})$} \\
\hline Small & 0.19 & 0.12 & 0.28 & 0.36 & 0.51 & 0.78 \\
\hline Medium & -0.86 & -0.29 & -0.33 & -2.18 & -0.98 & -1.05 \\
\hline \multirow[t]{2}{*}{ Big } & 0.49 & -0.32 & 0.19 & 2.99 & -0.82 & 0.58 \\
\hline & \multicolumn{3}{|c|}{$\mathrm{b}$} & \multicolumn{3}{|c|}{$t(b)$} \\
\hline Small & 1.26 & 1.00 & 0.97 & 12.76 & 15.07 & 21.91 \\
\hline Medium & 1.23 & 1.07 & 1.03 & 20.28 & 28.43 & 32.22 \\
\hline \multirow[t]{2}{*}{ Big } & 1.09 & 1.08 & 1.15 & 29.39 & 35.75 & 30.31 \\
\hline & \multicolumn{3}{|c|}{$\mathrm{S}$} & \multicolumn{3}{|c|}{$\mathrm{t}(\mathrm{s})$} \\
\hline Small & 1.54 & 1.20 & 1.30 & 11.42 & 13.28 & 21.44 \\
\hline Medium & 1.00 & 0.96 & 0.85 & 12.05 & 18.81 & 19.51 \\
\hline \multirow[t]{2}{*}{ Big } & 0.37 & 0.41 & 0.45 & 7.22 & 9.83 & 8.74 \\
\hline & \multicolumn{3}{|c|}{$\mathrm{h}$} & \multicolumn{3}{|c|}{$\mathrm{t}(\mathrm{h})$} \\
\hline Small & 0.66 & 0.39 & 0.64 & 3.66 & 3.21 & 7.85 \\
\hline Medium & 0.10 & 0.33 & 0.50 & 0.94 & 4.74 & 8.55 \\
\hline \multirow[t]{2}{*}{ Big } & -0.06 & 0.25 & 0.44 & -0.81 & 4.59 & 6.35 \\
\hline & \multicolumn{3}{|c|}{$\mathrm{c}$} & \multicolumn{3}{|c|}{$t(c)$} \\
\hline Small & -0.32 & 0.02 & 0.55 & -1.95 & 0.13 & 0.59 \\
\hline Medium & 0.15 & 0.10 & 0.17 & 0.95 & 1.03 & 1.53 \\
\hline \multirow[t]{2}{*}{ Big } & -0.21 & -0.08 & 0.09 & -2.91 & -1.37 & 0.98 \\
\hline & \multicolumn{3}{|c|}{$\mathrm{R} 2$} & \multicolumn{3}{|c|}{$\mathrm{s}(\mathrm{e})$} \\
\hline Small & 0.72 & 0.73 & 0.78 & 5.48 & 3.79 & 3.06 \\
\hline Medium & 0.75 & 0.66 & 0.62 & 4.34 & 4.23 & 4.84 \\
\hline Big & 0.84 & 0.72 & 0.69 & 2.55 & 3.14 & 3.66 \\
\hline
\end{tabular}


Table A2. (continued)

Corporate Liquidity Tertiles - Medium

\begin{tabular}{|c|c|c|c|c|c|c|}
\hline \multirow{3}{*}{ Size Tertiles } & \multicolumn{6}{|c|}{ Book-to-market Equity (BE/ME) Tertiles } \\
\hline & Low & Medium & High & Low & Medium & High \\
\hline & \multicolumn{3}{|c|}{$\mathrm{a}$} & \multicolumn{3}{|c|}{$\mathrm{t}(\mathrm{a})$} \\
\hline Small & -0.24 & -0.59 & 0.23 & -0.65 & -2.11 & 1.35 \\
\hline Medium & 0.26 & -0.28 & 0.38 & 0.84 & -0.68 & 1.45 \\
\hline \multirow[t]{2}{*}{ Big } & 0.22 & -0.47 & 0.19 & 0.76 & -1.43 & 0.67 \\
\hline & \multicolumn{3}{|c|}{$\mathrm{b}$} & \multicolumn{3}{|c|}{$\mathrm{t}(\mathrm{b})$} \\
\hline Small & 0.96 & 0.89 & 1.00 & 10.60 & 11.61 & 19.47 \\
\hline Medium & 1.09 & 1.08 & 1.10 & 15.47 & 27.40 & 29.36 \\
\hline \multirow[t]{2}{*}{ Big } & 1.01 & 1.06 & 1.06 & 28.33 & 32.80 & 25.22 \\
\hline & \multicolumn{3}{|c|}{$\mathrm{S}$} & \multicolumn{3}{|c|}{$\mathrm{t}(\mathrm{s})$} \\
\hline Small & 1.65 & 1.30 & 1.25 & 13.37 & 12.39 & 17.78 \\
\hline Medium & 1.13 & 0.91 & 0.98 & 11.71 & 16.91 & 19.14 \\
\hline \multirow[t]{2}{*}{ Big } & 0.35 & 0.33 & 0.48 & 7.11 & 7.53 & 8.33 \\
\hline & \multicolumn{3}{|c|}{$\mathrm{h}$} & \multicolumn{3}{|c|}{$\mathrm{t}(\mathrm{h})$} \\
\hline Small & 0.43 & 0.37 & 0.50 & 2.58 & 2.62 & 5.27 \\
\hline Medium & 0.02 & 0.13 & 0.43 & 0.13 & 1.84 & 6.20 \\
\hline \multirow[t]{2}{*}{ Big } & -0.20 & 0.27 & 0.41 & -3.00 & 4.49 & 5.34 \\
\hline & \multicolumn{3}{|c|}{$\mathrm{c}$} & \multicolumn{3}{|c|}{$t(c)$} \\
\hline Small & -0.11 & 0.03 & 0.15 & -0.72 & 0.27 & 1.67 \\
\hline Medium & 0.12 & 0.27 & 0.26 & 1.10 & 1.87 & 1.64 \\
\hline \multirow[t]{2}{*}{ Big } & -0.06 & -0.18 & 0.06 & -0.94 & -2.30 & 0.69 \\
\hline & \multicolumn{3}{|c|}{$\mathrm{R} 2$} & \multicolumn{3}{|c|}{$\mathrm{s}(\mathrm{e})$} \\
\hline Small & 0.71 & 0.70 & 0.77 & 5.29 & 4.63 & 3.34 \\
\hline Medium & 0.75 & 0.66 & 0.65 & 4.33 & 4.88 & 4.24 \\
\hline Big & 0.84 & 0.76 & 0.72 & 2.41 & 3.45 & 3.87 \\
\hline
\end{tabular}


Table A2. (continued)

Corporate Liquidity Tertiles - Liquid

\begin{tabular}{|c|c|c|c|c|c|c|}
\hline \multirow{3}{*}{ Size Tertiles } & \multicolumn{6}{|c|}{ Book-to-market Equity (BE/ME) Tertiles } \\
\hline & Low & Medium & High & Low & Medium & High \\
\hline & \multicolumn{3}{|c|}{$\mathrm{a}$} & \multicolumn{3}{|c|}{$\mathrm{t}(\mathrm{a})$} \\
\hline Small & -0.12 & -0.97 & 0.19 & -0.36 & -3.13 & 0.89 \\
\hline Medium & -0.29 & -0.44 & -0.07 & -0.94 & -1.40 & -0.28 \\
\hline \multirow[t]{2}{*}{ Big } & -0.16 & -0.49 & -0.02 & -0.62 & -1.96 & -0.04 \\
\hline & \multicolumn{3}{|c|}{$\mathrm{b}$} & \multicolumn{3}{|c|}{$\mathrm{t}(\mathrm{b})$} \\
\hline Small & 1.16 & 1.09 & 0.93 & 11.18 & 13.46 & 15.74 \\
\hline Medium & 1.17 & 1.10 & 0.90 & 16.03 & 21.69 & 16.30 \\
\hline \multirow[t]{2}{*}{ Big } & 0.91 & 1.07 & 0.91 & 13.85 & 18.16 & 19.37 \\
\hline & \multicolumn{3}{|c|}{$\mathrm{s}$} & \multicolumn{3}{|c|}{$\mathrm{t}(\mathrm{s})$} \\
\hline Small & 1.16 & 1.03 & 1.09 & 8.17 & 9.26 & 13.40 \\
\hline Medium & 0.99 & 0.79 & 0.93 & 9.88 & 11.43 & 12.23 \\
\hline \multirow[t]{2}{*}{ Big } & 0.59 & 0.35 & 0.37 & 6.60 & 4.32 & 5.69 \\
\hline & \multicolumn{3}{|c|}{$\mathrm{h}$} & \multicolumn{3}{|c|}{$\mathrm{t}(\mathrm{h})$} \\
\hline Small & 0.48 & 0.44 & 0.66 & 2.54 & 2.95 & 6.09 \\
\hline Medium & 0.21 & 0.25 & 0.43 & 1.55 & 2.73 & 4.22 \\
\hline \multirow[t]{2}{*}{ Big } & 0.04 & 0.17 & 0.24 & 0.30 & 1.59 & 2.75 \\
\hline & \multicolumn{3}{|c|}{$\mathrm{c}$} & \multicolumn{3}{|c|}{$\mathrm{t}(\mathrm{c})$} \\
\hline Small & 0.32 & 0.66 & 0.68 & 2.35 & 5.58 & 8.25 \\
\hline Medium & 0.91 & 0.64 & 0.71 & 8.50 & 5.32 & 6.98 \\
\hline \multirow[t]{2}{*}{ Big } & 0.69 & 0.57 & 0.27 & 8.98 & 6.61 & 1.85 \\
\hline & \multicolumn{3}{|c|}{$\mathrm{R} 2$} & \multicolumn{3}{|c|}{$\mathrm{s}(\mathrm{e})$} \\
\hline Small & 0.83 & 0.80 & 0.82 & 3.71 & 3.80 & 3.45 \\
\hline Medium & 0.84 & 0.79 & 0.65 & 3.22 & 4.76 & 3.98 \\
\hline Big & 0.88 & 0.81 & 0.64 & 2.64 & 3.23 & 4.43 \\
\hline
\end{tabular}

GRS test: $F$ value $=1.43$, -value $=0.09$ 
Table A3. The Fama-French Three-Factor + Corporate Liquidity Factors ( $L M I_{\text {med_low }}$ and $\left.L M I_{\text {high_med }}\right)$ on 27 Size-BE/ME-Corporate Liquidity Portfolios

Time series regressions of excess stock returns on the excess market returns $(M K T)$, the returns on size (SMB), book-to-market equity $(H M L)$ and the Corporate liquidity $\left(L M I_{\text {med_low }}, L M I_{\text {high_med }}\right)$ factors: July 1963 to December 2005, 510 months.

$$
R_{t}^{i}-R F_{t}=a^{i}+b^{i} M K T_{t}+s^{i} S M B_{t}+h^{i} H M L_{t}+c_{1}^{i} L M I_{\text {med_low }_{t}}+c_{2}^{i} L M I_{\text {high }_{-} \text {med }_{t}}+e_{t}
$$

Dependent variable: Excess returns on 25 stock portfolios formed on size, book-to-market equity and corporate liquidity.

Corporate Liquidity Tertiles - Illiquid

Book-to-market Equity (BE/ME) Tertiles

\begin{tabular}{|c|c|c|c|c|c|c|}
\hline \multirow{3}{*}{ Size Tertiles } & \\
\hline & Low & Medium & High & Low & Medium & High \\
\hline & \multicolumn{3}{|c|}{$\mathrm{a}$} & \multicolumn{3}{|c|}{$\mathrm{t}(\mathrm{a})$} \\
\hline Small & 0.37 & -0.15 & 0.41 & 0.89 & -0.45 & 1.45 \\
\hline Medium & -0.78 & -0.24 & 0.17 & -2.28 & -0.67 & 0.53 \\
\hline \multirow[t]{2}{*}{ Big } & 0.62 & -0.22 & 0.25 & 3.13 & -1.18 & 0.97 \\
\hline & \multicolumn{3}{|c|}{$\mathrm{b}$} & \multicolumn{3}{|c|}{$\mathrm{t}(\mathrm{b})$} \\
\hline Small & 1.27 & 1.00 & 0.98 & 12.90 & 15.09 & 22.27 \\
\hline Medium & 1.23 & 1.07 & 1.03 & 20.65 & 29.02 & 32.79 \\
\hline \multirow[t]{2}{*}{ Big } & 1.10 & 1.08 & 1.15 & 30.41 & 36.27 & 30.65 \\
\hline & \multicolumn{3}{|c|}{$\mathrm{s}$} & \multicolumn{3}{|c|}{$\mathrm{t}(\mathrm{s})$} \\
\hline Small & 1.53 & 1.20 & 1.29 & 11.38 & 13.23 & 21.54 \\
\hline Medium & 0.99 & 0.96 & 0.85 & 12.06 & 18.94 & 19.62 \\
\hline \multirow[t]{2}{*}{ Big } & 0.36 & 0.40 & 0.45 & 7.22 & 9.81 & 8.70 \\
\hline & \multicolumn{3}{|c|}{$\mathrm{h}$} & \multicolumn{3}{|c|}{$\mathrm{t}(\mathrm{h})$} \\
\hline Small & 0.58 & 0.37 & 0.59 & 3.19 & 3.00 & 7.18 \\
\hline Medium & 0.03 & 0.27 & 0.46 & 0.28 & 3.99 & 7.83 \\
\hline \multirow[t]{2}{*}{ Big } & -0.12 & 0.22 & 0.40 & -1.81 & 3.93 & 5.75 \\
\hline & \multicolumn{3}{|c|}{$\mathrm{c}_{1}$} & \multicolumn{3}{|c|}{$\mathrm{t}\left(\mathrm{c}_{1}\right)$} \\
\hline Small & -3.14 & -0.45 & -1.76 & -4.46 & -1.08 & -4.87 \\
\hline Medium & -1.28 & -1.18 & -1.26 & -3.26 & -2.56 & -2.87 \\
\hline \multirow[t]{2}{*}{ Big } & -0.81 & -0.89 & -1.06 & -2.95 & -2.65 & -2.56 \\
\hline & \multicolumn{3}{|c|}{$\mathrm{c}_{2}$} & \multicolumn{3}{|c|}{$\mathrm{t}\left(\mathrm{c}_{2}\right)$} \\
\hline Small & -0.32 & 0.01 & 0.01 & -2.27 & 0.05 & 0.25 \\
\hline Medium & 0.02 & 0.12 & 0.10 & 0.79 & 1.09 & 1.08 \\
\hline \multirow[t]{2}{*}{ Big } & 0.24 & -0.09 & -0.16 & 2.12 & -1.22 & -1.72 \\
\hline & \multicolumn{3}{|c|}{$\mathrm{R} 2$} & \multicolumn{3}{|c|}{$\mathrm{s}(\mathrm{e})$} \\
\hline Small & 0.76 & 0.73 & 0.80 & 4.37 & 4.56 & 2.86 \\
\hline Medium & 0.75 & 0.67 & 0.62 & 4.56 & 4.88 & 5.25 \\
\hline Big & 0.81 & 0.77 & 0.68 & 2.04 & 2.60 & 5.72 \\
\hline
\end{tabular}


Table A3. (continued)

Corporate Liquidity Tertiles - Medium

\begin{tabular}{|c|c|c|c|c|c|c|}
\hline \multirow[b]{2}{*}{ Size Tertiles } & \multicolumn{6}{|c|}{ Book-to-market Equity (BE/ME) Tertiles } \\
\hline & Low & Medium & High & Low & Medium & High \\
\hline & \multicolumn{3}{|c|}{$\bar{a}$} & \multicolumn{3}{|c|}{$\mathrm{t}(\mathrm{a})$} \\
\hline Small & -0.31 & 0.23 & 0.12 & -0.75 & 0.84 & 0.49 \\
\hline Medium & -0.34 & -0.36 & -0.32 & -1.99 & -1.28 & -1.07 \\
\hline \multirow[t]{2}{*}{ Big } & 0.38 & 0.34 & 0.14 & 1.89 & 1.43 & 0.56 \\
\hline & \multicolumn{3}{|c|}{$\mathrm{b}$} & \multicolumn{3}{|c|}{$\mathrm{t}(\mathrm{b})$} \\
\hline Small & 0.95 & 0.89 & 1.00 & 10.54 & 11.59 & 19.44 \\
\hline Medium & 1.09 & 1.08 & 1.10 & 15.47 & 27.35 & 29.31 \\
\hline \multirow[t]{2}{*}{ Big } & 1.01 & 1.06 & 1.06 & 28.27 & 32.75 & 25.16 \\
\hline & \multicolumn{3}{|c|}{$\mathrm{s}$} & \multicolumn{3}{|c|}{$\mathrm{t}(\mathrm{s})$} \\
\hline Small & 1.66 & 1.30 & 1.25 & 13.46 & 12.36 & 17.75 \\
\hline Medium & 1.12 & 0.91 & 0.98 & 11.67 & 16.88 & 19.10 \\
\hline \multirow[t]{2}{*}{ Big } & 0.35 & 0.33 & 0.48 & 7.11 & 7.51 & 8.35 \\
\hline & \multicolumn{3}{|c|}{$\mathrm{h}$} & \multicolumn{3}{|c|}{$\mathrm{t}(\mathrm{h})$} \\
\hline Small & 0.48 & 0.37 & 0.50 & 2.87 & 2.55 & 5.18 \\
\hline Medium & 0.00 & 0.13 & 0.42 & 0.02 & 1.84 & 6.05 \\
\hline \multirow[t]{2}{*}{ Big } & -0.19 & 0.26 & 0.42 & -2.92 & 4.38 & 5.38 \\
\hline & \multicolumn{3}{|c|}{$\mathrm{c}_{1}$} & \multicolumn{3}{|c|}{$\mathrm{t}\left(\mathrm{c}_{1}\right)$} \\
\hline Small & 0.28 & -0.09 & 0.37 & 1.05 & -0.12 & 1.46 \\
\hline Medium & -0.11 & -0.13 & -0.44 & -0.19 & -0.32 & -0.98 \\
\hline \multirow[t]{2}{*}{ Big } & -0.14 & -0.27 & 0.19 & -0.45 & -0.45 & 0.48 \\
\hline & \multicolumn{3}{|c|}{$c_{2}$} & \multicolumn{3}{|c|}{$\mathrm{t}\left(\mathrm{c}_{2}\right)$} \\
\hline Small & -0.09 & 0.03 & 0.11 & -0.65 & 0.26 & 1.28 \\
\hline Medium & 0.21 & 0.06 & 0.16 & 1.89 & 0.65 & 1.57 \\
\hline \multirow[t]{2}{*}{ Big } & -0.08 & -0.18 & -0.05 & -1.06 & -2.31 & -0.62 \\
\hline & \multicolumn{3}{|c|}{$\mathrm{R} 2$} & \multicolumn{3}{|c|}{$\mathrm{s}(\mathrm{e})$} \\
\hline Small & 0.71 & 0.70 & 0.72 & 5.31 & 3.62 & 3.18 \\
\hline Medium & 0.72 & 0.63 & 0.68 & 4.20 & 4.04 & 3.52 \\
\hline Big & 0.85 & 0.73 & 0.69 & 2.24 & 2.90 & 3.12 \\
\hline
\end{tabular}


Table A3. (continued)

Corporate Liquidity Tertiles - Liquid

\begin{tabular}{|c|c|c|c|c|c|c|}
\hline \multirow[b]{2}{*}{ Size Tertiles } & \multicolumn{6}{|c|}{ Book-to-market Equity (BE/ME) Tertiles } \\
\hline & \multirow[t]{2}{*}{ Low } & \multirow{2}{*}{\multicolumn{2}{|c|}{$\frac{\text { Medium }}{\mathrm{a}}$}} & Low & Medium & High \\
\hline & & & & \multicolumn{3}{|c|}{$\mathrm{t}(\mathrm{a})$} \\
\hline Small & -0.12 & -0.28 & -0.23 & -0.78 & -1.01 & -0.95 \\
\hline Medium & -0.96 & -0.36 & -0.49 & -2.96 & -1.22 & -1.66 \\
\hline \multirow[t]{2}{*}{ Big } & 0.19 & -0.09 & 0.08 & 0.91 & -0.44 & 0.25 \\
\hline & \multicolumn{3}{|c|}{$\mathrm{b}$} & \multicolumn{3}{|c|}{$\mathrm{t}(\mathrm{b})$} \\
\hline Small & 1.17 & 1.09 & 0.94 & 11.29 & 13.45 & 15.91 \\
\hline Medium & 1.18 & 1.10 & 0.91 & 16.14 & 21.65 & 16.31 \\
\hline \multirow[t]{2}{*}{ Big } & 0.90 & 1.07 & 0.92 & 13.81 & 18.11 & 19.41 \\
\hline & \multicolumn{3}{|c|}{$\mathrm{s}$} & \multicolumn{3}{|c|}{$\mathrm{t}(\mathrm{s})$} \\
\hline Small & 1.15 & 1.03 & 1.08 & 8.12 & 9.24 & 13.37 \\
\hline Medium & 0.98 & 0.79 & 0.92 & 9.83 & 11.41 & 12.19 \\
\hline \multirow[t]{2}{*}{ Big } & 0.60 & 0.35 & 0.36 & 6.72 & 4.33 & 5.65 \\
\hline & \multicolumn{3}{|c|}{$\mathrm{h}$} & \multicolumn{3}{|c|}{$\mathrm{t}(\mathrm{h})$} \\
\hline Small & 0.41 & 0.43 & 0.61 & 2.14 & 2.86 & 5.57 \\
\hline Medium & 0.16 & 0.25 & 0.42 & 1.19 & 2.68 & 4.01 \\
\hline \multirow[t]{2}{*}{ Big } & 0.09 & 0.18 & 0.22 & 0.76 & 1.66 & 2.50 \\
\hline & \multicolumn{3}{|c|}{$\mathrm{c}_{1}$} & \multicolumn{3}{|c|}{$\mathrm{t}\left(\mathrm{c}_{1}\right)$} \\
\hline Small & -0.53 & 0.76 & 0.34 & -0.79 & 2.02 & 1.06 \\
\hline Medium & 0.07 & -0.22 & -0.48 & 0.15 & -0.43 & -1.17 \\
\hline \multirow[t]{2}{*}{ Big } & -0.12 & 0.19 & -0.89 & -0.34 & 0.49 & -1.78 \\
\hline & \multicolumn{3}{|c|}{$\mathrm{c}_{2}$} & \multicolumn{3}{|c|}{$\mathrm{t}\left(\mathrm{c}_{2}\right)$} \\
\hline Small & 0.32 & 0.59 & 0.76 & 2.16 & 5.76 & 9.55 \\
\hline Medium & 0.83 & 0.79 & 0.64 & 7.52 & 7.28 & 6.92 \\
\hline \multirow[t]{2}{*}{ Big } & 0.68 & 0.54 & 0.19 & 8.84 & 6.56 & 1.87 \\
\hline & \multicolumn{3}{|c|}{$\mathrm{R} 2$} & \multicolumn{3}{|c|}{$\mathrm{s}(\mathrm{e})$} \\
\hline Small & 0.84 & 0.79 & 0.82 & 3.54 & 4.34 & 3.96 \\
\hline Medium & 0.83 & 0.86 & 0.64 & 3.78 & 3.14 & 4.87 \\
\hline Big & 0.87 & 0.81 & 0.66 & 2.92 & 3.98 & 4.12 \\
\hline
\end{tabular}

GRS test: $F$ value $=1.45, p$-value $=0.09$ 


\section{Table B. Returns on Zero-investment Portfolios Sorted by Size and BE/ME}

\section{Panel A. Long Liquid Firms and Short Illiquid Firms}

In each June and December, the entire sample is sorted into 9 size-BE/ME portfolios. Within each portfolio, all the stocks are further sorted into 2 portfolios: liquid portfolio, which includes firms whose corporate liquidity is ranked beyond top 30 percentiles; and illiquid portfolio, which includes firms whose corporate liquidity is ranked below bottom 30 percentiles. The 9 zero-investment portfolios are constructed as long the liquid firms and short the illiquid firms. Monthly portfolio returns are calculated as equally weighted average of individual stock returns. Finally geometric averages of cumulative portfolio returns for the 6 months following the month when the portfolios are formed (excluding the immediate subsequent month) are calculated.

\begin{tabular}{l|ccc} 
& low & medium & high \\
\hline small & 1.45 & 0.21 & 0.55 \\
& $(3.51)^{* * *}$ & $(0.41)$ & $(1.64)$ \\
medium & 0.38 & -0.21 & -0.43 \\
& $(0.75)$ & $(-0.46)$ & $(-1.14)$ \\
big & 0.40 & -0.37 & -0.44 \\
& $(0.76)$ & $(-1.00)$ & $(-0.92)$ \\
\hline
\end{tabular}

\section{Panel B. Long Medium Liquid Firms and Short Illiquid Firms}

In each June and December, the entire sample is sorted into 9 size-BE/ME portfolios. Within each portfolio, all the stocks are further sorted into 2 portfolios: medium liquid portfolio, which includes firms whose corporate liquidity is ranked as medium percentiles (40-70 percentiles); and illiquid portfolio, which includes firms whose corporate liquidity is ranked below bottom 30 percentiles. The 9 zero-investment portfolios are constructed as long the medium liquid firms and short the illiquid firms. Monthly portfolio returns are calculated as equally weighted average of individual stock returns. Finally geometric averages of accumulative portfolio returns for the 6 months following the month when the portfolios are formed (excluding the immediate subsequent month) are calculated.

\begin{tabular}{l|ccc} 
& low & medium & high \\
\hline small & 0.94 & 0.08 & 0.60 \\
& $(2.15)^{* *}$ & $(0.29)$ & $(3.69)^{* * *}$ \\
medium & 0.47 & 0.17 & 0.20 \\
& $(1.28)$ & $(0.99)$ & $(1.20)$ \\
big & 0.44 & 0.03 & 0.25 \\
& $(1.60)$ & $(0.20)$ & $(1.40)$ \\
\hline
\end{tabular}

\section{Panel C. Long Liquid Firms and Short Medium Liquid Firms}

In each June and December, the entire sample is sorted into 9 size-BE/ME portfolios. Within each portfolio, all the stocks are further sorted into 2 portfolios: liquid portfolio, which includes firms whose corporate liquidity is ranked above top 30 percentiles; and medium liquid portfolio, which includes firms whose corporate liquidity is ranked as medium percentiles (40-70 percentiles). The 9 zero-investment portfolios are constructed as long the liquid firms and short the medium liquid firms. Monthly portfolio returns are calculated as equally weighted average of individual stock returns. Finally geometric averages of accumulative portfolio returns for the 6 months following the month when the portfolios are formed (excluding the immediate subsequent month) are calculated.

\begin{tabular}{l|ccc} 
& low & medium & high \\
\hline small & 0.51 & 0.13 & -0.05 \\
& $(1.18)$ & $(0.32)$ & $(-0.20)$ \\
medium & -0.09 & -0.37 & -0.63 \\
& $(-0.32)$ & $(-1.13)$ & $(-2.08)^{* *}$ \\
big & -0.04 & -0.40 & -0.68 \\
& $(-0.13)$ & $(-1.32)$ & $(-1.71)^{*}$ \\
\hline
\end{tabular}

$* 90 \%$ significance level $* * 95 \%$ significance level $* * * 99 \%$ significance level 


\section{Chapter 3}

\section{Residual Cash, Firm Value and Financial Distress}

\section{Introduction}

The first two chapters link the cash holding level as a measure of the corporate liquidity to the equity returns, and examine whether corporate liquidity is an idiosyncratic risk or a systematic risk factor. According to tradeoff theory, firms that aim to maximize shareholders' wealth should maintain the cash holding at an optimal level where the marginal benefit of keeping financial slack should equal the marginal cost. Any deviation from the target level of cash holding will lower the value of the firm. However, in the real business world, firms tend to hold more or less cash than the "target" or "normal" level. It is tempting to look at the "residual cash" or "excess cash" - deviation from the target cash level and relate it to future equity returns, growth and investment opportunities, operating performance and the probability of financial distress and bankruptcy.

Most previous papers studying cash holding deviating from an "optimal" or "normal" level focus on the positive portion of residual cash. Since excessively high liquidity is associated with agency problems ${ }^{19}$, the literature addresses the positive residual cash issue together with firm control and corporate governance. Opler, Pinkowitz, Stulz and Williamson (1999) investigate the expenditure pattern for both positive and negative residual cash firms and they find that firms with positive residual cash conduct higher capital expenditure than negative residual cash firms, which can be interpreted as due to a financial constraint for negative residual cash firms. They do not find evidence to support the agency theory. Faleye (2004) looks at the take-over market and finds that proxy-fight targets keep more cash than non-target firms. He also shows that cash holdings decline substantially after the proxy contest. Sibilkov (2005) finds that constrained firms hold negative residual cash because they are financially constrained, not because of the agency

\footnotetext{
${ }^{19}$ Myers and Rajan (1998) argue that corporate liquidity can be a source of agency problems. Dittmar, Mahrt-Smith and Servaes (2003) claim that agency problems are an important determinant of corporate cash holdings, and find that corporations in countries with poor shareholder protections hold more cash than countries where shareholder rights are well protected. Pinkowitz, Stulz and Williamson (2005) use cross country data to test the agency theory. They find that minority shareholders value cash holdings less in countries where investor protection is poor than in other countries, which provides supportive evidence for the argument that agency problems play an important role in firm valuation across countries.
} 
problems. Dittmar and Mahrt-Smith (2007) show that corporate governance impacts the firm value substantially through the channel of cash. They find that excess cash is more valuable for firms with good corporate governance than poorly governed firms. They also find that poorly governed companies dissipate cash quickly and in a value-destroying manner. Pinkowitz and Williamson (2004) estimate the market value of cash held by firms. They find that cash is more valuable for firms with good growth options while is less valuable in firms with stable investment programs, easier access to capital and those nearer financial distress.

Previous related literature finds that firms with positive excess cash tend to undertake more capital expenditure than negative residual cash companies. Moreover, positive excess cash will increase the firm's market value only under sound corporate governance. Managers tend to squander free cash flows due to the agency problem, therefore, firms with good corporate governance tend to invest cash in a more efficient manner and increase shareholders' value. As such, positive excess cash holding might increase future equity returns if the firm have good growth opportunities and good corporate governance. Managers of poorly governed firms are more likely to squander cash in some negative NPV projects if there are no profitable investment projects. One common proxy for future growth opportunities is the market-to-book ratio. We would expect that positive residual cash holding firms with a higher market-to-book ratio have higher future stock returns compared to similar firms but with lower market-to-book ratios.

On the other side, negative residual cash holding companies are more likely to be financially constrained (Sibilkov (2005)). Negative residual cash could be the result of bad recent performance, or of management strategy. If a firm has negative residual cash temporarily but has a high market-to-book ratio, the future market value of the firm can be increased based on better performance. On the contrary, if the firm's negative cash is persistent, and if it does not have good investment opportunities to improve its performance, negative cash might be a predictor of default or bankruptcy.

This chapter examines the role of residual cash on affecting companies' market value, especially the negative part of the residual cash. The empirical results show that firms with positive excess cash holdings have positive excess returns on assets, equity and investment as well as cash flows on average while firms with negative residual cash 
holdings have excess negative return on assets, equity and investment. If the poor performance of the negative residual cash firms continues over time and if this is the reason that these firms actually have a consistently lower level of corporate liquidity compared to the "normal" level, the market value of these firms will be dampened. In addition, compared to those with positive excess cash, firms with negative residual cash tend to be smaller, spend more in research and development, distribute less to shareholders and lack good governance. Furthermore, it can be shown that the average net financing is much greater for negative residual cash firms while the average interest coverage ratio is substantially higher for positive excess cash firms than negative residual cash firms, which indicates a higher default risk for the latter group. As such, we can project that the negative residual cash might be useful as an indicator of financial default risk and bankruptcy.

I also investigate the ability of corporate liquidity and negative residual cash to predict bankruptcy. In addition, whether the residual cash contributes to the various default probability measures developed by previous literature is studied. Logistic regression results indicate that negative residual cash is an important predictor variable to forecast bankruptcy. Firms with negative residual cash are more likely to go bankruptcy in the future. Negative residual cash also contributes to different default probability measures estimated in previous literature, including Altman's Z-score, Vassalou and Xing's default likelihood indicator $(D L I)$ and Chava and Jarrow's default probability.

The remaining of this paper is organized as follows. Section II discusses the hypothesis to be tested. Section III introduces the dataset and variables used in the empirical estimation. Section IV discusses the approach that is used to estimate the normal cash and residual cash. Section V describes the empirical results from the value regression and the logistic regression. Section VI concludes.

\section{Hypotheses}

Previous literature has intensively studied the role of corporate cash holding, the relationship between cash holding and various firm characteristics. According to tradeoff theory, holding liquid asset brings benefits to firms, and these benefits also serve as 
motives for holding cash; however, maintaining asset liquidity incurs costs as well. There should be an optimal level of liquid asset holdings on a corporate balance sheet that can maximize the stockholder's value (Kim, Mauer and Sherman (1998) and Opler, Pinkowitz, Stulz and Williamson (1999)). Any deviation from the optimal level will reduce the market value of the company. On the other hand, Mikkelson and Partch (2003) find that high cash holding companies tend to invest more and grow faster in assets. Their empirical results show that conservative financial policies actually support investment and do not hinder performance. Sibilkov (2005) argues that the reason why firms keep cash reserves below the normal level is because they are financially constrained. In other words, compared to negative residual cash holding companies, companies that maintain corporate liquidity above the normal level are not subject to a financial constraint and are more likely to invest and improve operating performance. If firms accumulate cash more than necessary for normal business operation because they are better managed, they have shown higher profits and better performance, the market price of the stocks for positive excess cash holding firms will incorporate this information and their market value will be higher than those with corporate liquidity lower than the normal level.

Hypothesis 1a: According to the tradeoff theory, there exists an optimal level of cash which can maximize shareholders' value. Hence, the market value of equity decreases as the absolute value of residual cash increases.

Hypothesis 1b: Market value of equity falls less as the absolute value of residual cash increases, if the residual cash is positive.

The above hypothesis suggests two variables in the empirical value regression: the absolute value of residual cash and a dummy variable for negative residual. Both positive and negative deviation from optimal or normal cash level can be value destroying, however the destructive effect of positive residual cash will be smaller. This asymmetric nonlinear relationship between residual cash and the market value of equity can be illustrated by the graph below: 


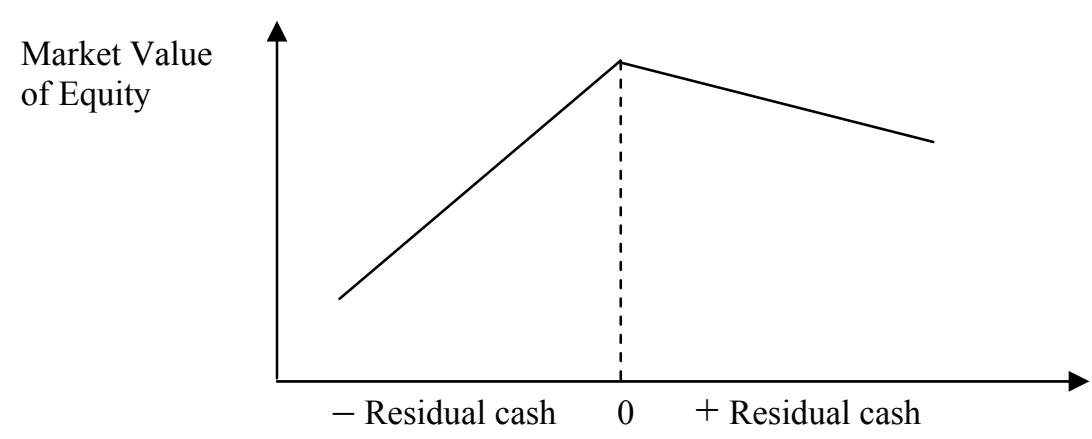

The second hypothesis deals with the firms with corporate liquidity less than the "normal" cash level. Negative residual cash holding firms are more likely to be subject to financial distress risk if this situation persists for a relatively long period of time. Altman (1968) uses working capital as one of the variables in the discriminant function to predict bankruptcy. Zmijewski (1984) includes the current ratio as an explanatory variable in the bankruptcy prediction model. Kim, Mauer and Sherman (1998) show that firms holding a lower level of liquidity tend to have a higher probability of financial distress. Campbell, Hilscher and Szilagyi (2005) find that corporate cash is an important variable when predicting corporate bankruptcies and failures. Furthermore, Sibilkov (2005) find that firms with negative residual cash are more likely to be financially constrained, which indicates that these firms have higher default risk if they have high financial leverage or volatile cash flows, or macroeconomic conditions are weak. Firms holding negative residual cash while performing poorly over time have a higher probability of default and bankruptcy. Such firms keep a lower level of corporate liquidity compared to normal not because of management strategy, but due to the poor management.

Hypothesis 2: Firms with more negative residual cash holdings are more likely subject to financial distress risk and have a higher bankruptcy probability.

Hypothesis 1 is tested in part V-1 and hypothesis 2 is tested in part V.2 and V.3. 


\section{Data}

The sample consists of all US publicly traded firms from 1950 to 2004 where the necessary data items are available. Financial statement data are derived from the COMPUSTAT Industrial Annual Research files and the stock and indices databases from the Center for Research in Security Prices (CRSP). The CRSP returns cover NYSE, AMEX and NASDAQ stocks. I exclude financial firms with Standard Industrial Classification (SIC) codes between 6000 and 6999 because they usually hold substantial inventories of marketable securities, which are included in cash. Transportation and public utility firms with SIC codes between 4000 and 4999 are excluded as well because cash holdings of these firms are subject to regulatory supervision.

As the risk free interest rate for the asset pricing model, I use monthly observations of the one year Treasury bill rate obtained from Kenneth French's webpage. ${ }^{20}$ Market excess returns are downloaded from Kenneth French's webpage as well.

Following Kim, Mauer and Sherman (1998), I measure corporate liquidity $(C L)$ as the ratio of cash and marketable securities to total assets:

$$
C L_{i t}=\frac{\text { Cash }_{i t}}{\text { Total Assets }_{i t}}
$$

where $\operatorname{Cash}_{i t}$ refers to cash and cash equivalents including: bank drafts, cash, checks (cashiers or certified), demand certificates of deposit, demand deposits, letters of credit and money orders. It also includes government and other marketable securities and time deposits, which are usually considered as cash equivalents. A firm with a high cash-toasset ratio has more liquid assets to serve interest payments, and can therefore postpone bankruptcy and reduce financial distress; or has more freedom to invest in profitable projects without incurring transaction costs. Alternatively, corporate liquidity is measured by the ratio of cash and cash equivalents to net assets, where net assets equal total assets less cash and cash equivalents (Dittmar, Mahrt-Smith and Servaes (2003), Faleye (2004), etc.). I also follow Campbell, Hilscher and Szilagyi (2005) and measure liquidity as cash, marketable securities and short term investment divided by total assets, since short term investment is often quite liquid as well. Empirical results are similar when the two alternative measures of corporate liquidity are used.

\footnotetext{
${ }^{20} \mathrm{http}: / /$ mba.tuck.dartmouth.edu/pages/faculty/ken.french
} 
To reduce the impact of outliers, firm specific characteristics and other dependent variables are winsorized at the 1 percent and 99 percent tails of the entire sample. Firms with negative total assets, book value and market value of equity are deleted from the sample.

Several default probability measures from the literature are used in the current study. The first measure is Altman's Z-score which is a widely accepted classic measure of financial distress. Altman $(1968)^{21}$ uses accounting variables to predict the probability of bankruptcy. The second measure is the default likelihood indicator (DLI) created by Vassalou and Xing (2005), which measures the default probability that a firm's total assets are less than its total liabilities. This dataset is made available by Maria Vassalou on her website ${ }^{22}$. Third, I use the bankruptcy hazard rate constructed by Chava and Jarrow (2004). They calculate the bankruptcy probability based on Shumway (2001)'s hazard rate model, but using a comprehensive bankruptcy database which consists of all bankruptcy filings reported in the Wall Street Journal Index (1962 -1980), the SDC Database (Reorganization module 1980 - 1999), SEC filings (1978 - 1999) and the CCH Capital Changes Reporter.

For the actual bankruptcy and liquidation data, I collect the events over the sample period from the COMPUSTAT Research file and footnotes and CRSP delisting codes. Firms with a CRSP bankruptcy delisting code 400-490 (liquidations), 572 (liquidations) and 574 (bankruptcy) and firms with COMPUSTAT footnote 35 having code 02 (filing for Chapter 11) or 03 (Chapter 7) are considered as failing and will be included in the bankruptcy sub sample. There are 874 delistings and around 94695 firm years in the sample.

Kothari, Shanken and Sloan (1995) point out that COMPUSTAT has the "back-filling" problem, which creates the survivor bias. Specifically, when COMPUSTAT adds a firm to its data file, it often "back-fills" data for several previous years. For example, if a firm

\footnotetext{
${ }^{21}$ Altman's Z-score is calculated as follows: $\mathrm{Z}=.012 \mathrm{X}_{1}+.014 \mathrm{X}_{2}+.033 \mathrm{X}_{3}+.006 \mathrm{X}_{4}+.999 \mathrm{X}_{5}$, where $\mathrm{X}_{1}=$ Working capital/Total assets

$\mathrm{X}_{2}=$ Retained earnings/Total assets

$\mathrm{X}_{3}=$ Earnings before interest and taxes/Total assets

$\mathrm{X}_{4}=$ Market value equity/Book value of total debt

$\mathrm{X}_{5}=$ Sales/Total assets

$\mathrm{Z}=$ Over all index

${ }^{22} \mathrm{http}: / /$ www2.gsb.columbia.edu/faculty/mvassalou/data.html
} 
is added in 1985, COMPUSTAT might fill in data for the firm back to 1980. In this way, COMPUSTAT is more likely to include distressed firms that ultimately survive and to exclude distressed firms that fail. However, Fama and French (1996) argue that the problem of survivor bias in merged data from COMPUSTAT and CRSP is only of minor importance.

In order to minimize the impact of the "back-filling" problem La Porta, Lakonishok, Shleifer and Vishny (1997), firms with less than 5 years of COMPUSTAT data available are eliminated from the sample. Moreover, since my sample is generated by merging COMPUSTAT and CRSP, the survivor bias problem is of less concern.

\section{Estimation of Residual Cash}

As in the previous literature (Faleye (2004), Dittmar and Mahrt-Smith (2007)), excess cash is defined as the portion of cash kept above the "optimal" level of cash, and is not necessary to maintain normal operations or investment. Furthermore, I also define the negative residual cash as the shortage of cash needed to keep the "optimal" or "target" level. Optimal cash level can be estimated using a regression of cash holdings on a group of variables that proxy theoretically justified reasons for holding cash: transaction costs, investment opportunities, riskiness of cash flows, access to external financing, etc. Based on Opler, Pinkowitz, Stulz and Williamson (1999) and Dittmar and Mahrt-Smith (2007), I estimate the "normal" or "optimal" cash holdings by the following equation:

$$
\begin{aligned}
& \text { Corporate Liqudity }_{i, t}=\alpha+\beta_{1} \text { Assets }_{i, t}+\beta_{2} \frac{C F_{i, t}}{\text { Assets }_{i, t}}+\beta_{3} \frac{N W C_{i, t}}{\text { Assets }_{i, t}}+\beta_{4} M_{\text {MTtoBK }} \\
& +\beta_{5} \frac{\text { CapitalExpense }_{i, t}}{\text { Assets }_{i, t}}+\beta_{6} \frac{R \& D_{i, t}}{\text { Sales }_{i, t}}+\beta_{7} \text { Leverage }_{i, t} \\
& +\beta_{8} \text { Dividend }_{i, t}+\beta_{9} \text { Bond Rating }_{i, t}+\beta_{10} G-\text { Index }_{i, t} \\
& + \text { Year Dummies }+ \text { Firm Fixed Effects }+\varepsilon_{i, t}
\end{aligned}
$$

The variables (COMPUSTAT item number in parentheses) in the above equation are: Corporate Liquidity measured as cash and marketable securities (1) to total assets (6); real size (Assets), measured by total assets deflated to 2000 dollars using the GDP deflator; cash flow $(C F)$, measured as earnings before interest, taxes and depreciation (13) 
minus taxes (16) minus interest (15), divided by total assets; net working capital (NWC) measured as current assets (4) minus cash and cash equivalents (1) current liabilities (5), divided by total assets; the market to book ratio $(M K T t o B K)$ measured as the fiscal year end market price (CRSP data) times the number of shares outstanding (CRSP data) plus total liabilities (181), divided by book value of total assets; capital expenses (CapitalExpense) measured by capital expenditure (128) by total assets; $R \& D^{23}$, measured by research and development expenses (46) divided by sales (12); Leverage, measured by the ratio of long term debt (9) plus short term debt (34) divided by total assets; Dividend, a dummy variable equal to one if the firm pays dividends (127); Bond Rating, the S\&P long term bond rating with the lower score representing better creditability (280); G-Index, the Gompers et al. (2003) corporate governance index that measures the degree of managerial entrenchment due to takeover protection ${ }^{24}$. Year Dummies are included in the regression to control for the time-varying macroeconomic factors that can affect the firms' decision of cash holding. Finally, firm fixed effects are included to control for idiosyncratic reasons due to which some firms hold a high level of liquid assets such as Chrysler, Ford and Microsoft. Industry effect is not controlled when estimating the residual cash since it might be more of an individual firm issue, not widely exists in certain industries.

Residual cash is calculated as the difference between the actual cash held by the firms and the "optimal" or "normal" cash holdings predicted by the above regression equation. Faleye (2004), Dittmar and Mahrt-Smith (2007) look only at the positive excess cash, as their primary goal is to examine the impact of corporate governance on the value and/or quantity of excess cash holdings. I would like to investigate the negative part of excess cash, which can be related to financial distress, and even future bankruptcy. Based on the models estimated by Shumway (2001) and Chava and Jarrow (2004), Campbell, Hilscher and Szilagyi (2005) employ a relatively atheoretical logistic model to predict the probability that a firm goes bankruptcy or fails. The independent variables used to predict corporate failure are cash holdings, leverage, profitability, market to book ratio, market

\footnotetext{
${ }^{23} R \& D$ is set to zero where the values are missing.

${ }^{24}$ Gompers, Ishii and Metrick (2003) create the corporate governance index using the data from the Investor Responsibility Research Center (IRRC), which measures the number of anti-takeover provisions in a firm's charter and in the legal code of the state where the firm is incorporated. The index has a value range between 0 and 24, with more anti-takeover provisions indicating poorer corporate governance.
} 
capitalization, past stock returns, and past return volatility. They find that low cash holding companies are more likely to file for bankruptcy. Since high level of cash holdings are more closely related to corporate governance issue due to the agency problem, it is the low cash holding situation, namely, negative residual cash holdings relative to the normal level, that should more precisely predict bankruptcy.

Column 1 of Table 1 displays the results of a reduced form regression of the residual cash estimation. Larger firms hold significantly less cash, which is consistent with an economy of scale explanation. Furthermore, firm size is often used as a proxy for the ability to get access to external capital market. The result shows that on average, larger firms can relatively easily raise external funds and therefore hold less financial slack. Firms accumulate cash from cash flows, and hence we can observe a positive relationship between cash holding and cash flow. Net working capital is considered as a substitute for cash and cash equivalents. The regression results show that firm with higher net working capital as an alternative source of funds do not maintain a high level of corporate liquidity. Growth opportunities are proxied by the market-to-book ratio. Firms with greater investment opportunities tend to hold more cash for speculative reasons.

Column 2 includes two additional variables. The bond rating is used to account for access to the external financial market. Firms with a better long term bond rating (lower score in the S\&P bond rating) hold more cash. Recent research finds that corporate governance affects companies' decisions to hold financial liquidity (Dittmar, MahrtSmith and Servaes (2003), and Harford, Mansi and Maxwell (2005)), I therefore include the G-Index in the normal cash regression to account for this effect. It turns out firms under good corporate governance (a lower score on the G-Index) keep a lower level of cash, which is consistent with the previous literature. Following the specification of Dittmar and Mahrt-Smith (2007), I further include capital expenditure, R\&D expenses, leverage and dividend payments in the estimation of normal cash holding. A great amount of capital expenditure draws down current corporate liquidity, as can be seen from the negative coefficient on capital expenses. Firms with substantial research and development expenses are more subject to an information asymmetry problem, with hence higher external capital cost, and therefore a need to keep more liquid assets. Leverage is negatively associated with corporate liquidity, which is consistent with the 
pecking order or financial hierarchy theory according to which firms accumulate cash to pay back their debt when it comes due. In general, cash rich companies tend to distribute more dividends to their shareholders, as is confirmed by the negative sign on the coefficient of the dividend variable.

Alternatively, excess cash can be estimated by subtracting some benchmark cash holding level from individual corporate liquidity. One benchmark can be the industry-size average cash holding since previous literature shows corporate liquidity varies depending on industry and size. First, all the firms are categorized into 10 industry portfolios defined by Fama and French; then within each industry portfolio, firms are subsequently sorted into 5 size sub portfolios based on market equity. Next, the industry-size corporate liquidity norm is calculated each period by taking the average of cash holdings of all firms falling into a certain industry-size portfolio.

\section{Empirical Results}

Panel A of Table 2 compares the summary statistics for firms with positive and negative residual cash, which are residuals from the normal cash regressions. Contemporaneous annual equity returns are similar for positive and negative residual cash firms, but positive excess cash firms have higher one-year future equity returns. On average, firms with positive excess cash are smaller in terms of real assets. Firms with cash holdings lower than the "normal" level have higher market-to-book ratios and substantially greater research and development expenditures. This implies that the negative residual cash firms have more growth opportunities, which is consistent with Dittmar and Mahrt-Smith (2007). The possible interpretation is that growth firms spend a substantial amount of cash to finance investment and end up with a lower level of corporate liquidity. However the fact that their value is more dependent on growth opportunities and intangible assets makes them more likely to suffer a severe asymmetric information problem and be financially constrained (Myers and Majluf (1984)). Net working capital for positive excess cash companies is significantly more abundant than for negative residual cash companies, with net working capital as a proportion of total assets equal to 9.8 percent and 0.8 percent respectively. 
The current cash flow over total assets ratio is 0.102 for positive excess cash firms but -0.062 for negative residual cash firms. The previous cash flow over total assets ratio is 0.103 and -0.206 for positive and negative residual cash companies respectively. The 1 year future cash flow - total asset ratio for positive excess cash firms is slightly higher at 0.118, while cash flow for negative residual cash firms increases substantially to 0.054 . This indicates that firms with positive excess cash typically generate stable profit streams as well as cash flows, while negative residual cash companies may have investment opportunities but their operating performance is not stable, and their cash flows are relatively more volatile, which, may affect their solvency. The return on assets is 0.042 and -0.105 for firms that have higher- and lower-than-normal cash level respectively. Similarly, the return on equity and the return on investment are positive for positive excess cash firms while they are negative for negative residual cash firms. It seems that the negative residual cash firms have very low profit and this is probably the reason they have low financial slack for the current period.

Negative residual cash firms raise more external capital than their positive excess cash counterparts, with the net finance equal to 10.3 percent compared to 1.8 percent of total assets. However their ability to make interest payments is quite low, as the interest coverage ratio for negative residual cash firms is only -1.033 while positive excess cash companies have a much stronger ability to pay back their debt.

For the dividend paid to shareholders, it can be shown that the payout ratio of negative residual cash firms is less than half of the positive excess cash firms. In addition, distribution to shareholders ${ }^{25}$ shows the same pattern. Previous literature uses the payout ratio as one of the financial constraint measures. It can be argued that firms without sufficient cash to maintain normal needs are subject to financial constraints, which is consistent with Sibilkov (2005).

Panel B of Table 2 shows the summary statistics of firm characteristics of positive and negative residual cash holding companies using industry and firm size as benchmarks. The results are similar to Panel A.

Figure 1a displays the time series of the mean and median of normal cash holdings and excess cash holdings derived from the regression approach. It can be shown that average

\footnotetext{
${ }^{25}$ Distribution to shareholders is calculated as dividend plus stock repurchase divided by total assets.
} 
normal cash holdings are relatively stable through 1999, while showing an upward trend after that, with peaks in 2000 and 2004 slightly higher than 15 percent and 17 percent respectively. The cross sectional mean of excess cash is around zero throughout the sample period, with a spike of 3 percent in 1997. Median normal cash and median excess cash are lower than the means, which indicates that the distributions of both normal and excess cash are right skewed.

Figure $1 \mathrm{~b}$ shows the mean and median of normal and excess cash holding estimated subtracting industry-size portfolio average from individual corporate liquidity. The sample size is much larger for this method because we do not use variables like G-index which have limited availability. The time series exhibition covers the period from 1950 to 2004. The level of normal cash holding derived using the industry-size portfolio approach is higher than that derived from the regression method. It can be shown that there is peak in cash holding around 1954 followed by a sharp drop after that. The normal cash holding is relatively stable after 1958 with the bottom equal to 8.5 percent in 1974. After 1991, the normal cash holdings increase sharply on average and reaches 24.3 percent at the end of 2004. The mean excess cash is relatively stable over time around zero while the median excess cash is declining steadily.

\section{V.1. The Impact of Residual Cash on Firm Value}

One important question related to the corporate liquidity deviating from the normal level is: whether and how does excess cash affect the market value of firms' equity? Following the literature (Pinkowitz and Williamson (2004), Dittmar and Mahrt-Smith (2007), etc.), I employ a valuation regression similar to the model developed by Fama and French (1998). Lagged corporate liquidity and changes in corporate liquidity are included as additional variables to identify the impact of corporate liquidity on firm value. The baseline specification of the value regression is as follows: 


$$
\begin{aligned}
& \frac{M V_{i, t}}{\text { Assets }_{i, t}}=\alpha+\beta_{1} \frac{E_{i, t}}{\text { Assets }_{i, t}}+\beta_{2} \frac{\Delta E_{i, t}}{\text { Assets }_{i, t}}+\beta_{3} \frac{\Delta N A_{i, t}}{\text { Assets }_{i, t}}+\beta_{4} \frac{\Delta N A_{i, t+1}}{\text { Assets }_{i, t}}+\beta_{5} \frac{R \& D_{i, t}}{\text { Assets }_{i, t}} \\
& +\beta_{6} \frac{\Delta R \& D_{i, t}}{\text { Assets }_{i, t}}+\beta_{7} \frac{{I N T_{i, t}}_{\text {Assets }_{i, t}}}{\text { Assets }_{i, t}}+\beta_{8} \frac{\Delta I N T_{i, t}}{\text { Assidend }_{i, t}}+\beta_{10} \frac{\text { Divividend }_{i, t}}{\text { Assets }_{i, t}} \\
& +\beta_{11} \frac{\Delta M V_{i, t+1}}{\text { Assets }_{i, t}}+\beta_{12} \text { Leverage }_{i, t}+\beta_{13} \frac{N F_{i, t}}{\text { Assets }_{i, t}}+\beta_{14} \frac{C L_{i, t-1}}{\text { Assets }_{i, t}}+\beta_{15} \frac{\Delta C L_{i, t}}{\text { Assets }_{i, t}} \\
& + \text { Year Dummies }+ \text { Firm Fixed Effects }+\varepsilon_{i, t}
\end{aligned}
$$

where $X_{t}$ (COMPUSTAT item number in parenthesis) indicates the level of the variable $X$ at time $t$ and $\Delta X_{t}$ indicates the changes in the variable $X$ from time $t-1$ to t. $M V$ is the market value of total assets, measured as the fiscal year end market price (CRSP data) times the number of shares outstanding (CRSP data) plus total liabilities (181). $E$ is the earnings before extraordinary items $(18+15+50+51)$. $N A$ is net assets, measured as total assets (6) minus cash and cash equivalents (1). $R \& D$ is research and development expenditure (46), which is set to zero wherever the value is missing. INT is the interest expenses (15). Dividend is dividend distributed to common shareholders (21). Leverage is calculated as the ratio of total liabilities $(9+34)$ to total assets. $N F$ is the net financing, measured as total equity issuance (108) minus stock repurchases (115) plus debt issuance (111) minus debt redemption (114). Table 3 shows the results of the baseline regression and several variants. Year dummies and fixed firm effects are controlled.

Column 1 presents the empirical results of the baseline value regression. It can be shown that a higher level of corporate liquidity significantly increases the firm's market value on average. The change in the level of cash holding over the last period is negatively associated with the firm's value.

In order to further examine the role of excess cash, several more value regressions with additional variables are estimated and the results are presented in column 2 through column 6 in Table 3. The regression in column 2 includes the interaction term Corporate Liquidity $_{t-1} *$ Positive Dummy $_{t-1}$, which tries to capture the additional value effect imposed by holding corporate liquidity beyond the "normal" level. Positive Dummy is a dummy variable equal to one if the residual cash estimated from equation (1) is positive and zero 
otherwise. It turns out that holding positive excess cash will further increase the firm's market value significantly.

To decompose the role of normal cash holding and excess cash holding in affecting the firm's market value, instead of regressing on the level of corporate liquidity, normal cash holding and absolute value of residual cash holding enter the value regression separately. According to tradeoff theory, there should be an "optimal" level of cash holding where the shareholders' wealth can be maximized. Column 3 tests this hypothesis by including the absolute value of excess cash holding to the regression. The coefficient of the absolute excess cash should be negative since any deviation from the optimal level will be harmful for the firm's value. The result supports the tradeoff theory. Column 4 adds the squared absolute excess cash as an additional variable. The impact of residual cash on firm value is quadratic.

Furthermore, in order to test the Hypothesis $1 \mathrm{~b}$, which asks whether positive and negative residual cash affects the market value of a firm differently, a negative residual cash dummy variable and an interaction term of the absolute value of residual cash and a negative dummy are included in the value regression. Column 5 and column 6 show the empirical results. It turns out that both the coefficients on the positive dummy and the interaction term are significant and positive. Given the fact that residual cash will reduce the market value of a firm, positive residual cash reduces the firm value less than negative residual cash.

\section{V.2. Negative Residual Cash and the Probability of Bankruptcy}

In order to examine the relationship between negative residual cash and the probability of bankruptcy and failure, I follow Shumway (2001), Chava and Jarrow (2004) and Campbell, Hilscher and Szilagyi (2005) and estimate a logit model.

$$
P_{t-1}\left(y_{i t}=1\right)=\frac{1}{1+\exp \left(-\alpha-\beta x_{i, t-1}\right)},
$$

where $y_{i t}$ is an bankruptcy indicator that equals 1 if the firm bankrupts or fails during year $t$ and $x_{i t}$ includes standard variables used in bankruptcy prediction models: net income (NI/TA), total liabilities to total assets (Leverage), excess stock returns (ExRet), stock return volatility $(S I G M A)$, relative size (RSIZE) defined as the logarithm of firms' market 
equity value to the market capitalization of the S\&P 500 index, the market to book ratio $(M K T t o B K)$, stock price (PRICE) and cash holding to total assets $\left(C_{-} T A\right)$. In order to examine the predictive power of residual cash, I add the interaction term of $C_{-} T A$ and a dummy variable which equals 1 if residual cash is below zero.

Furthermore, the overall cash holding position is decomposed into normal cash and residual cash to investigate their roles in predicting future bankruptcy and failure respectively. It is particularly interesting to see the how the absolute value of residual cash and negative cash holding can affect the bankruptcy probability. Specifically, if tradeoff theory is correct, and there is an optimal cash level at which shareholders' value is maximized, then any deviation from this optimal level will decrease the firm's value and therefore increase the probability of failure. In addition, to examine whether firms with positive residual cash are less likely subject to default risk, a negative residual cash dummy and an interaction term of absolute value of residual cash and negative residual cash dummy are included in the logistic regression. All predictors are lagged one period, so that they are known at the beginning of the period during which bankruptcy or failure occurs. According to the logistic regression, a higher value of $\left(\alpha+\beta x_{i, t-1}\right)$ contributes to a higher probability of bankruptcy or failure. If negative residual cash helps forecast bankruptcy of firms, we would expect that the coefficient on the negative residual cash dummy as well as the coefficient on the interaction term should be significantly positive.

Table 4 presents the number of bankruptcies and failures, the total number of active firms and the percentage of bankruptcies each year during the sample period $1950-2005$. There are a total of 874 bankruptcies in the sample, which includes all bankruptcy filings as reported in the COMPUSTAT and CRSP datasets. Firms with a CRSP bankruptcy delisting code 400-490 (liquidations), 572 (liquidations) and 574 (bankruptcy), and firms with COMPUSTAT footnote 35 having code 02 (Chapter 11) or 03 (Chapter 7) are considered as failing and will be included in the bankruptcy sub sample. Furthermore, Figure 2 shows the number of bankruptcies per year over the sample period and Figure 3 exhibits the bankruptcies as a percentage of active firms by year. As can be seen, there was no bankruptcy or failure before 1966. The bankruptcy filings increase substantially over the 1970s, the late 1980s and the second half of 1990s. The early 1990s and 2000s observe the dramatically decreased bankruptcies. 
Table 5 presents the logistic regression estimation for various model specifications using data from 1950 - 2005. The estimated coefficients, a chi-square value of each coefficient and measures of model fit are reported. The standard predictor variables enter the logit model with correct sign and substantial significance. For instance, a lower level of net income implies a higher probability of bankruptcy, while a greater market capitalization relative to the S\&P 500 market value will reduce the chance of going bankruptcy. If a firm relies on heavily borrowing, or its stock returns are highly volatile, or the excess equity returns are lower during the previous year, the likelihood of bankruptcy or failure increases.

Model 3 includes cash holding as an additional predictor in the logit model. Similar to Campell, Hilscher and Szilagyi (2005), the coefficient is significantly negative, which implies that keeping a higher level of corporate liquidity helps reduce the probability of the bankruptcy or at least postpone it. Model 4 uses the two parts of decomposed cash holding: normal cash and absolute value of residual cash as two separate prediction variables. It turns out the forecast ability of cash concentrates in the residual cash, which has a negative coefficient, and is highly significant at the 1 percent level. On the other hand, the normal part of corporate liquidity enters the logistic model insignificantly. Furthermore, the coefficient on the negative dummy is positive and significant, indicating that firms with negative residual cash has a higher bankruptcy risk. Model 5 shows that the interaction term of absolute value of residual cash and the negative residual cash dummy has a positive sign. Given the corporate liquidity position, holding a less-thannormal-level of cash will increase the chance to go bankrupt.

To better interpret the impact of residual cash on the possibility of bankruptcy, I compute the marginal effects for several independent variables at the sample mean. It turns out that normal cash holdings have a marginal effect of -0.064 , while the marginal effect of the absolute value of residual cash on the bankruptcy possibility is 0.078 . Higher normal corporate liquidity helps reduce the probability of going bankrupt; however, a deviation from this normal level in either direction will increase the chance of bankruptcy on average. In particular, a one percent increase in the absolute value of residual cash will increase the probability of bankruptcy by 7.8 percent at the margin. In addition, the negative residual cash dummy imposes a positive marginal impact equal to 0.026 on the 
bankruptcy probability, implying that firms with corporate liquidity lower than the target level are three percent more likely to file for bankruptcy. Finally, the marginal effect of the interaction term of the absolute value of residual cash and the negative residual cash dummy is calculated to be equal to 0.019 , which indicates that, given a constant level of the absolute value of residual cash, negative residual cash holdings increase the probability of bankruptcy at the margin by 0.2 percent.

\section{V.3. Negative Residual Cash and Financial Distress}

The main purpose of this chapter is to address the issue whether negative residual cash can be used as an indicator to predict future financial distress and bankruptcy. We have seen in section V-2 that negative residual cash can help predict bankruptcy. In this section, I investigate the relationship between negative residual cash and the possibility of financial distress using various measures of default probability.

I perform various regressions of the probability of financial default and bankruptcy on firm characteristics including lagged negative residual cash to investigate whether negative residual cash plays a role in forecasting bankruptcy. The three default probability measures are Altman's Z-score, Vassalou and Xing's default likelihood indicator $(D L I)$, and Chava and Jarrow's default probability. Altman' Z-score is a bankruptcy predictor that determines the likelihood of a firm going bankrupt by a multivariate formula combining five common business ratios, with a weighting system calculated by Altman. Studies show that the Z-score is usually accurate in predicting bankruptcy. However, such models using only accounting data are considered backward looking because accounting information only provides summary of a company's past performance rather than the future prospects. Vassalou and Xing (2004) use Black and Scholes (1973) contingent claims methodology and Merton's (1974) option pricing model to calculate the default measure Default Likelihood Indicator (DLI) using equity data. They find that DLI contains very different information from the aggregate default spreads between BAA corporate bonds and U.S. Treasury bonds, and it well predicts actual defaults. The last default likelihood measure is the bankruptcy probability constructed by Chava and Jarrow (2004). They use the bankruptcy hazard rate model and an expanded bankruptcy database to estimate the probability of bankruptcy and failure. 
The firm characteristics used as independent variables include: net income (NI/TA), total liabilities to total assets (Leverage), excess stock returns (ExRet), stock return's volatility (SIGMA), relative size (RSIZE) defined as the logarithm of firms' market equity value to the market capitalization of S\&P 500 index, market to book ratio (MKTtoBK), stock price (PRICE) and cash holding to total assets (C_TA), normal cash holding, absolute value of residual cash holding, and the interaction term between absolute value of residual cash holding or negative residual cash dummy.

The empirical results are presented in Table 6. Panel A shows the regression estimation with Altman's Z-score as the dependent variable. All the firm characteristics enter the regression model with a significant coefficient. Consistent with other literature, a higher earning, lower leverage, higher excess stock return, lower equity return volatility, larger size and more corporate liquidity help reduce the default probability. Given the corporate liquidity level, holding negative residual cash tend to increase the chance of default, as we observe either the negative dummy or the interaction term between absolute value of residual corporate liquidity and the negative residual cash dummy has a negative sign. Panel B uses Vassalou and Xing's default likelihood indicator as the dependent variable. The estimation shows that the coefficient on cash holding is positive but not significant; in addition, holding less-than-normal cash increases the default likelihood, given the corporate liquidity level. When the cash holding is decomposed into normal and residual part, it turns out that the normal cash remains insignificant while the coefficient of absolute value of residual cash is positive and highly significant, which implies that firms should stick to the optimal level of cash to reduce distress risk. Finally, compared to holding positive excess cash, firms with negative residual cash tend to have higher probability of default, everything else equal, as we can see from the negative coefficient on the negative dummy and interaction term of absolute value of residual cash and negative dummy. Panel $\mathrm{C}$ summarizes the results for Chava and Jarrow's default probability, and the results are similar to that of $D L I$.

\section{V.4. Forecasting Regression of Residual cash}

If residual cash of time $t$ contains some information about the firm's near future, it is possible that residual cash can predict the returns, cash flows, investment, profitability 
and probability of financial distress or bankruptcy for the period $t+1$ but not for further period $t+2$. Similarly, residual cash of time $t+1$ can provide some information to predict firm returns, cash flows and other variables for the period $t+2$ but not for from period $t+3$ on. However, variables like equity returns, cash flows, profitability at a specific point of time are fairly noisy, which can be affected by a lot of factors other than residual cash. For this reason, current residual cash may not forecast stock returns and other firm characteristics accurately for the immediate future. If residual cash is persistent, then residual cash in period $t$ is closed related to residual cash in period $t+1$, and has some predictive power for the stock returns in period $t+2$. This implies that residual cash holding for period $t$ can provide a much better prediction for future stock returns, cash flows, investment, profitability, etc. at a longer time horizon if the firm's stock is held for a longer period because in the long run, noises in stock returns and other firm characteristics are diversified and averaged out.

The forecasting ability of residual cash can be estimated using the following two regressions for various holding horizons:

$$
\begin{aligned}
& X_{t, t+h}-\bar{X}_{t, t+h}=\alpha+\beta_{1} \mid \text { Residual Cash }{ }_{t} \mid+\beta_{2} \text { Negative Dummy } y_{t}+\varepsilon_{t} \\
& X_{t, t+h}-\bar{X}_{t, t+h}=\alpha+\beta_{1} \mid \text { Residual Cash } h_{t}\left|+\beta_{2}\right| \text { Residual Cas } h_{t} \mid \times \text { Negative Dummy }_{t}+\varepsilon_{t},
\end{aligned}
$$

where $X_{t, t+h}$ is the variable to be predicted over the holding period from $t$ to $t+h$, and $\bar{X}_{t, t+h}$ is the benchmark of $X_{t, t+h} . X_{t, t+h}$ to be forecasted here includes: $R_{t, t+h}$, cumulative stock returns over the holding period from $t$ to $t+h ; R O A_{t, t+h}$, average return on assets over the period $t$ to $t+h$; Residual $c a s h_{t, t+h}$, average residual cash different from the normal cash holding level estimated by equation (1) during period $t$ to $t+h ; R \& D_{t, t+h}$, average research and development expenditure in period $t$ to $t+h ; C F_{t, t+h}$, average cash flows during period $t$ to $t+h$; and Capital Expense $_{t, t+h}$, average capital investment over period $t$ to $t+h$.

Benchmark value $\bar{X}_{t, t+h}$ is measured as the industry-size average of $X_{t, t+h}$. First, in each period, all the firms are categorized into industry portfolios according to SIC code; then within each industry portfolio, firms are subsequently sorted into 5 size sub portfolios. Next, the industry-size norm of the variable $X_{t, t+h}$ is calculated each period by taking the 
average of $X$ of all firms falling into a certain industry-size portfolio over the period $t$ to $t+h$.

The results of the above regressions are presented in Table 7. It turns out that residual cash indeed contains some information to predict the firm characteristics. A change in residual cash from the optimal level predicts that the stock return as well as the net income and cash flows will drop over the next $2-3$ years. Compared to those with negative residual cash, the future returns, net income and cash flows of firms with positive residual cash fall less. Furthermore, an increase in residual cash is related to an increase in research and development expenses and capital expenditure over the next several years, implying that firms are piling up an extra amount of liquid assets for investment purposes. Moreover, residual cash tends to be highly persistent over time, which indicates that managers tend to keep a relatively stable cash and working capital policy

\section{Conclusion}

This chapter examines the role of residual cash, especially the negative part of the residual cash, on affecting companies' market value, financial distress and bankruptcy probability. Normal cash and residual cash are estimated using a regression approach and a portfolio approach. Firm characteristics are studied for positive and negative residual cash portfolios respectively. The empirical results show that deviation from the target or optimal cash is value destructive, which provides support to the tradeoff theory. However, market value of equity falls less as the absolute value of residual cash increases, if the residual cash is positive. Compared to those with positive residual cash, firms with negative residual cash tend to be smaller, generate lower cash flows and profits, borrow more external capital, have weaker ability to pay off debt, spend more on research and development, distribute less amount to shareholders and lack good governance.

I also investigate the capability of corporate liquidity and negative residual cash to forecast the bankruptcy. Logistic regression results indicate that negative residual cash serves as an important predictor variable to forecast bankruptcy. Firms with negative residual cash are more likely to go bankrupt in the future. In addition, the issue whether 
the residual cash contributes to the various default probability measures developed by previous literature has been studied. It can be shown that negative residual cash increases the default probability as measured by Altman's Z-score, Vassalou and Xing's default likelihood indicator and Chava and Jarrow's default probability. Finally, by regressing the residual cash on various firm characteristics, we can see that residual cash indeed contains information to predict firm characteristics over the future years. 


\section{REFERENCE}

Almeida, Heitor, Murillo Campello, and Michael S. Weisbach, 2004, The Cash Flow Sensitivity of Cash, Journal of Finance 59, 1777-1804.

Altman, Edward I., 1968, Financial Ratios, Discriminant Analysis and the Prediction of Corporate Bankruptcy, Journal of Finance 23, 589-609.

Bodie, Zvi, Alex Kane, and Alan J. Marcus, 1999, Investments, $4^{\text {th }}$ edition, McGraw-Hill College.

Campbell, Y., John, Jens Hilscher, and Jan Szilagyi, 2005, In Search of Distress Risk, working paper, Harvard University.

Chava, Sudheer, and Robert A. Jarrow, 2004, Bankruptcy Prediction with Industry Effects, Review of Finance 8, 537-569.

Dittmar, Amy, and Jan Mahrt-Smith, 2007, Corporate Governance and the Value of Cash Holding, Journal of Financial Economics 83, 599-634.

Dittmar, Amy, Jan Mahrt-Smith, and Henri Servaes, 2003, International Corporate Governance and Corporate Cash Holdings, Journal of Financial and Quantitative Analysis, Special Edition on International Corporate Governance, 38, 111-134.

Faleye, Olububmi, 2004, Cash and Corporate Control, Journal of Finance 59, 2041-2060.

Fama, Eugene F., and Kenneth French, 1998, Taxes, Financing Decisions, and Firm Value, Journal of Finance 53, 819-843.

Gompers, Paul A., Joy L. Ishii, and Andrew Metrick, 2003, Corporate Governance and Equity Prices, Quarterly Journal of Economics 118, 107-155.

Kothari, S. P., Jay Shanken, and Richard G. Sloan, 1995, Another Look at the CrossSection of Expected Returns, Journal of Finance 50, 185-224.

La Porta, Rafael, Josef Lakonishok, Andrei Shleifer, and Robert Vishny, 1997, Good News for Value Stocks: Further Evidence on Market Efficiency, Journal of Finance $52,859-874$.

Mikkelson, Wayne, and Megan Partch, 2003, Do Persistent Large Cash Reserve Hinder Performance? Journal of Financial and Quantitative Analysis 38, 275-294.

Myers, Stewart C., and Raghuram G. Rajan, 1998, The Paradox of Liquidity, Quarterly Journal of Economics 113, 733-771. 
Opler, Tim, Lee Pinkowitz, Rene Stulz, and Rohan Williamson, 1999, The Determinants and Implications of Corporate Cash Holdings, Journal of Financial Economics 52, 346.

Pinkowitz, Lee, and Rohan Williamson, 2004, What is a Dollar Worth? The Market Value of Cash Holdings, working paper, Georgetown University.

Pinkowitz, Lee, René Stulz, and Rohan Williamson, 2005, Does the Contribution of Corporate Cash Holdings and Dividends to Firm Value Depend on Governance? A Cross-country Analysis, Journal of Finance 61, 2725-2751.

Sibilkov, Valeriy, 2005, Is Cash More Valuable for Constrained Firms? working paper, Purdue University.

Vassalou, Maria, and Yuhang Xing, 2004, Default Risk in Equity Returns, Journal of Finance 59, 831-868.

Zmijewski, Mark E., 1984, Methodology Issues Related to the Estimation of Financial Distress Prediction Models, Journal of Accounting Research 22, 59-82. 


\section{Table 1. Predicting the Normal Level of Cash}

This table displays the regression results for the normal level of cash. The dependent variable is Corporate Liquidity, measured as the ratio of cash divided by total assets. The independent variables include: real size (Assets), measured by total assets deflated to 2000 dollars using the GDP deflator; cash flow $(C F)$, measured as earnings before interest, taxes and depreciation minus taxes minus interest, divided by total assets; net working capital $(N W C)$ measured as current assets minus current liabilities, divided by total assets; market to book ratio (MKTtoBK) measured as the fiscal year end market price times the number of shares outstanding plus the total liabilities, divided by book value of total assets; capital expenses measured by capital expenditure by total assets; $R \& D$, measured by research and development expenses divided by sales; leverage, measured by the ratio of long term debt plus short term debt divided by total assets; Dividend, a dummy variable equal to one if the firm pays dividends; Bond Rating, measured as a dummy variable equal to one if the firm has a corporate bond rating; G-Index, the Gompers et al. (2003) corporate governance index that measures the degree of managerial entrenchment due to takeover protection. All ratios are winsorized at the $1 \%$ and $99 \%$ tails. All models are estimated as OLS regression with Year dummies and firm fixed effects. tstatistics are included in the parenthesis.

\begin{tabular}{|c|c|c|c|}
\hline & (1) & (2) & (3) \\
\hline Constant & $\begin{array}{c}0.154 \\
(140.89)^{* * *}\end{array}$ & $\begin{array}{c}0.278 \\
(53.13)^{* * *}\end{array}$ & $\begin{array}{c}0.336 \\
(28.78)^{* * *}\end{array}$ \\
\hline Assets $_{t}$ & $\begin{array}{c}-0.224 \\
(-20.43)^{* * *}\end{array}$ & $\begin{array}{c}-0.131 \\
(-7.66)^{* * *}\end{array}$ & $\begin{array}{c}-0.249 \\
(-6.20)^{* * *}\end{array}$ \\
\hline $\mathrm{CF}_{\mathrm{t}} /$ Assets $_{\mathrm{t}}$ & $\begin{array}{c}0.016 \\
(10.66)^{* * *}\end{array}$ & $\begin{array}{c}0.326 \\
(27.44)^{* * *}\end{array}$ & $\begin{array}{c}0.161 \\
(10.98)^{* * *}\end{array}$ \\
\hline $\mathrm{NWC}_{\mathrm{t}} /$ Assets $_{\mathrm{t}}$ & $\begin{array}{c}-0.056 \\
(-21.50)^{* * *}\end{array}$ & $\begin{array}{c}-0.273 \\
(-30.94)^{* * *}\end{array}$ & $\begin{array}{c}-0.307 \\
(-27.59)^{* * *}\end{array}$ \\
\hline MKTtoBK $_{t}$ & $\begin{array}{c}0.013 \\
(55.12)^{* * * *}\end{array}$ & $\begin{array}{c}0.018 \\
(33.39)^{* * *}\end{array}$ & $\begin{array}{c}0.010 \\
(19.52)^{* * *}\end{array}$ \\
\hline CapitalExpense $_{t} /$ Assets $_{t}$ & - & - & $\begin{array}{c}-0.693 \\
(-19.27)^{* * *}\end{array}$ \\
\hline $\mathrm{R} \& \mathrm{D} /$ Assets $_{\mathrm{t}}$ & - & - & $\begin{array}{c}0.476 \\
(18.14)^{* * *}\end{array}$ \\
\hline Leverage $_{t}$ & - & - & $\begin{array}{c}-0.243 \\
(-27.27)^{* * *}\end{array}$ \\
\hline Dividend $_{t}$ & $\begin{array}{l}- \\
-\end{array}$ & $\begin{array}{l}- \\
-\end{array}$ & $\begin{array}{c}-0.055 \\
(-15.29)^{* * *}\end{array}$ \\
\hline Bond Rating $_{t}$ & - & $\begin{array}{c}0.009 \\
(58.43)^{* * *}\end{array}$ & $\begin{array}{c}0.008 \\
(58.12)^{* * *}\end{array}$ \\
\hline G-Index $x_{t}$ & $\begin{array}{l}- \\
-\end{array}$ & $\begin{array}{c}-0.008 \\
(-14.26)^{* * *}\end{array}$ & $\begin{array}{c}-0.06 \\
(-13.86)^{* * *}\end{array}$ \\
\hline Adjusted $R^{2}$ & 0.09 & 0.30 & 0.54 \\
\hline
\end{tabular}




\section{Table 2. Summary Statistics for Firms with Positive and Negative Residual Cash Holdings}

\section{Panel A}

This table compares the summary statistics of characteristics of firms with positive residual cash and negative residual cash. Positive and negative residual cash is calculated as the positive part and negative part of the residuals from the normal cash regression. The variables are: contemporaneous equity returns (Returns $s_{t}$ ), expected equity returns $\left(\right.$ Returns $_{t+1}$ ), estimated normal cash (Normal Cash), estimated residual cash (Residual cash), real assets (Real Assets), market-to-book ratio (MKTtoBK), ratio of capital expense to assets (CapitalExpense/Assets), ratio of research to sales (R\&D/Sales), leverage (Leverage), corporate liquidity for previous period (Corporate Liquidity $t_{t-1}$ ), current period (Corporate Liquidity $t$ ), and next period (Corporate Liquidity $\mathrm{t}_{\mathrm{t}}$ ), ratio of cash flow over assets (CF/Assets), book-to-market ratio (BE/ME), ratio of net working capital to assets (NWC/Assets), return on assets (ROA), return on equity (ROE), return on investment (ROI), amount of net finance raised in current period (Net Finance), interest coverage (INT Coverage), payout ratio (Payout Ratio), distribution to shareholders (Distribution to Shareholder $\mathrm{r}_{\mathrm{t}}$, and Gompers et al. index $\left(\mathrm{G}-\mathrm{Index}_{\mathrm{t}}\right)$. All variables are winsorized at $1 \%$ and $99 \%$ tails.

\begin{tabular}{|c|c|c|c|c|}
\hline & \multicolumn{2}{|c|}{ Mean } & \multicolumn{2}{|c|}{ Median } \\
\hline & $\begin{array}{c}\text { Positive } \\
\text { Residual cash }\end{array}$ & $\begin{array}{c}\text { Negative } \\
\text { residual cash }\end{array}$ & $\begin{array}{c}\text { Positive } \\
\text { Residual cash }\end{array}$ & $\begin{array}{c}\text { Negative } \\
\text { residual cash }\end{array}$ \\
\hline Returns $_{t}$ & $\begin{array}{c}0.177 \\
(0.829)\end{array}$ & $\begin{array}{c}0.173 \\
(0.904)\end{array}$ & 0.058 & 0.054 \\
\hline Returns $_{t+1}$ & $\begin{array}{c}0.209 \\
(0.975)\end{array}$ & $\begin{array}{c}0.172 \\
(0.902)\end{array}$ & 0.069 & 0.054 \\
\hline Normal Cash $_{t}$ & $\begin{array}{c}0.123 \\
(0.102)\end{array}$ & $\begin{array}{c}0.087 \\
(0.072)\end{array}$ & 0.103 & 0.067 \\
\hline Residual cash $h_{t}$ & $\begin{array}{c}0.078 \\
(0.124)\end{array}$ & $\begin{array}{l}-0.047 \\
(0.043)\end{array}$ & 0.052 & -0.037 \\
\hline Real Assets $_{t}$ & $\begin{array}{c}58.052 \\
(124.338)\end{array}$ & $\begin{array}{c}10.009 \\
(61.427)\end{array}$ & 20.852 & 0.745 \\
\hline MKTtoBK $_{t}$ & $\begin{array}{c}2.033 \\
(1.266)\end{array}$ & $\begin{array}{c}2.369 \\
(13.230)\end{array}$ & 1.661 & 1.327 \\
\hline CapitalExpense/Assets & $\begin{array}{c}0.068 \\
(0.060)\end{array}$ & $\begin{array}{c}0.075 \\
(0.159)\end{array}$ & 0.052 & 0.048 \\
\hline$R \& D /$ Sales $_{t}$ & $\begin{array}{c}0.036 \\
(0.123)\end{array}$ & $\begin{array}{c}0.801 \\
(16.991)\end{array}$ & 0.002 & 0 \\
\hline Leverage $_{t}$ & $\begin{array}{c}0.299 \\
(0.212)\end{array}$ & $\begin{array}{c}0.304 \\
(3.197)\end{array}$ & 0.266 & 0.202 \\
\hline Corporate Liquidity & $\begin{array}{c}0.172 \\
(0.134)\end{array}$ & $\begin{array}{c}0.085 \\
(0.120)\end{array}$ & 0.089 & 0.077 \\
\hline Corporate Liquidity & $\begin{array}{c}0.201 \\
(0.147)\end{array}$ & $\begin{array}{c}0.040 \\
(0.117)\end{array}$ & 0.104 & 0.053 \\
\hline Corporate Liquidity $_{t+1}$ & $\begin{array}{c}0.212 \\
(0.146)\end{array}$ & $\begin{array}{c}0.069 \\
(0.111)\end{array}$ & 0.097 & 0.067 \\
\hline$C F /$ Assets $_{t-1}$ & $\begin{array}{c}0.103 \\
(0.082)\end{array}$ & $\begin{array}{c}-0.206 \\
(16.721)\end{array}$ & 0.083 & 0.058 \\
\hline CF/Assets ${ }_{t}$ & $\begin{array}{c}0.102 \\
(0.084)\end{array}$ & $\begin{array}{l}-0.062 \\
(2.566)\end{array}$ & 0.103 & 0.067 \\
\hline$C F /$ Assets $_{t+1}$ & $\begin{array}{c}0.118 \\
(0.095)\end{array}$ & $\begin{array}{c}0.054 \\
(2.389)\end{array}$ & 0.112 & 0.076 \\
\hline
\end{tabular}


Table 2. Panel A (continued)

\begin{tabular}{|c|c|c|c|c|}
\hline & \multicolumn{2}{|c|}{ Mean } & \multicolumn{2}{|c|}{ Median } \\
\hline & $\begin{array}{c}\text { Positive } \\
\text { Residual cash }\end{array}$ & $\begin{array}{c}\text { Negative } \\
\text { residual cash }\end{array}$ & $\begin{array}{c}\text { Positive } \\
\text { Residual cash }\end{array}$ & $\begin{array}{c}\text { Negative } \\
\text { residual cash }\end{array}$ \\
\hline$B E / M E_{t}$ & $\begin{array}{c}0.425 \\
(0.571)\end{array}$ & $\begin{array}{c}0.648 \\
(43.295)\end{array}$ & 0.376 & 0.557 \\
\hline$N W C /$ Assets $_{t}$ & $\begin{array}{c}0.098 \\
(0.138)\end{array}$ & $\begin{array}{c}0.008 \\
(3.113)\end{array}$ & 0.082 & 0.110 \\
\hline$R O A_{t}$ & $\begin{array}{c}0.042 \\
(0.067)\end{array}$ & $\begin{array}{l}-0.105 \\
(2.771)\end{array}$ & 0.042 & 0.027 \\
\hline$R O E_{t}$ & $\begin{array}{c}0.354 \\
(3.826)\end{array}$ & $\begin{array}{c}-0.068 \\
(21.580)\end{array}$ & 0.138 & 0.046 \\
\hline$R O I_{t}$ & $\begin{array}{c}0.118 \\
(0.191)\end{array}$ & $\begin{array}{l}-0.003 \\
(7.687)\end{array}$ & 0.116 & 0.092 \\
\hline Net Finance $_{t}$ & $\begin{array}{c}0.018 \\
(0.117)\end{array}$ & $\begin{array}{c}0.103 \\
(0.662)\end{array}$ & 0 & 0.005 \\
\hline INT Coverage $_{t}$ & $\begin{array}{c}8.595 \\
(28.245)\end{array}$ & $\begin{array}{c}-1.033 \\
(389.738)\end{array}$ & 3.897 & 2.345 \\
\hline Payout Ratio $_{t}$ & $\begin{array}{c}0.524 \\
(5.206)\end{array}$ & $\begin{array}{c}0.209 \\
(8.764)\end{array}$ & 0.170 & 0 \\
\hline $\begin{array}{l}\text { Distribution to } \\
\text { Shareholders }_{t}\end{array}$ & 0.050 & 0.021 & 0.025 & 0 \\
\hline G-Index & $\begin{array}{c}(0.183) \\
8.677 \\
(2.72)\end{array}$ & $\begin{array}{c}(0.095) \\
9.922 \\
(2.56)\end{array}$ & 10.000 & 9.000 \\
\hline
\end{tabular}


Table 2. (continued)

\section{Panel B}

This table compares the summary statistics of characteristics of firms with positive residual cash and negative residual cash. Positive and negative residual cash is calculated as the positive part and negative part of the difference between the individual firm's cash holding and the average industry-size portfolio cash holdings. The variables are: contemporaneous equity returns (Returns $s_{t}$ ), expected equity returns $\left(\right.$ Returns $_{\mathrm{t}+1}$ ), estimated normal cash (Normal Cash), estimated residual cash (Residual cash), real assets (Real Assets), market-to-book ratio (MKTtoBK), ratio of capital expense to assets (CapitalExpense/Assets), ratio of research to sales (R\&D/Sales), leverage (Leverage), corporate liquidity for previous period (Corporate Liquidity $\mathrm{t}_{\mathrm{t}-1}$ ), current period (Corporate Liquidity), and next period (Corporate Liquidity $_{\mathrm{t}+1}$ ), ratio of cash flow over assets $(\mathrm{CF} /$ Assets), book-to-market ratio (BE/ME), ratio of net working capital to assets (NWC/Assets), return on assets (ROA), return on equity (ROE), return on investment (ROI), amount of net finance raised in current period (Net Finance), interest coverage (INT Coverage), payout ratio (Payout Ratio), distribution to shareholders (Distribution to Shareholder $\mathrm{t}_{\mathrm{t}}$ ), and Gompers et al. index $\left(\mathrm{G}-\right.$ Index $\left._{\mathrm{t}}\right)$. All variables are winsorized at $1 \%$ and $99 \%$ tails.

\begin{tabular}{|c|c|c|c|c|}
\hline \multirow[b]{3}{*}{ Returns $_{t}$} & \multicolumn{2}{|c|}{ Mean } & \multicolumn{2}{|c|}{ Median } \\
\hline & $\begin{array}{c}\text { Positive } \\
\text { Residual cash }\end{array}$ & $\begin{array}{c}\text { Negative } \\
\text { residual cash }\end{array}$ & $\begin{array}{c}\text { Positive } \\
\text { Residual cash }\end{array}$ & $\begin{array}{c}\text { Negative } \\
\text { residual cash }\end{array}$ \\
\hline & $\begin{array}{c}0.177 \\
(0.841)\end{array}$ & $\begin{array}{c}0.165 \\
(0.991)\end{array}$ & 0.051 & 0.054 \\
\hline Returns $_{t+1}$ & $\begin{array}{c}0.169 \\
(0.809)\end{array}$ & $\begin{array}{c}0.177 \\
(1.103)\end{array}$ & 0.050 & 0.060 \\
\hline Normal Cash & $\begin{array}{c}0.180 \\
(0.105)\end{array}$ & $\begin{array}{c}0.157 \\
(0.091)\end{array}$ & 0.136 & 0.122 \\
\hline Residual cash $t_{t}$ & $\begin{array}{c}0.173 \\
(0.156)\end{array}$ & $\begin{array}{l}-0.099 \\
(0.073)\end{array}$ & 0.123 & -0.080 \\
\hline Real Assets $t_{t}$ & $\begin{array}{c}6.914 \\
(48.827)\end{array}$ & $\begin{array}{c}12.907 \\
(123.625)\end{array}$ & 5.260 & 7.589 \\
\hline MKTtoBK $_{t}$ & $\begin{array}{c}3.009 \\
(16.173)\end{array}$ & $\begin{array}{c}1.926 \\
(10.068)\end{array}$ & 1.667 & 1.231 \\
\hline CapitalExpense/Assets ${ }_{t}$ & $\begin{array}{c}0.071 \\
(0.239)\end{array}$ & $\begin{array}{c}0.078 \\
(0.093)\end{array}$ & 0.045 & 0.051 \\
\hline$R \& D /$ Sales $_{t}$ & $\begin{array}{c}0.052 \\
(0.250)\end{array}$ & $\begin{array}{l}0.206 \\
(2.658)\end{array}$ & 0.043 & 0.101 \\
\hline Leverage $_{t}$ & $\begin{array}{c}0.156 \\
(0.516)\end{array}$ & $\begin{array}{c}0.367 \\
(2.155)\end{array}$ & 0.057 & 0.270 \\
\hline Corporate Liquidity $_{t-1}$ & $\begin{array}{c}0.251 \\
(0.282)\end{array}$ & $\begin{array}{c}0.602 \\
(1.265)\end{array}$ & 0.197 & 0.038 \\
\hline Corporate Liquidity $_{t}$ & $\begin{array}{c}0.354 \\
(0.220)\end{array}$ & $\begin{array}{c}0.059 \\
(0.528)\end{array}$ & 0.299 & 0.037 \\
\hline Corporate Liquidity $_{t+1}$ & $\begin{array}{c}0.365 \\
(0.235)\end{array}$ & $\begin{array}{c}0.082 \\
(0.952)\end{array}$ & 0.255 & 0.043 \\
\hline$C F /$ Assets $_{t-1}$ & $\begin{array}{c}0.070 \\
(0.532)\end{array}$ & $\begin{array}{l}-0.056 \\
(1.260)\end{array}$ & 0.052 & 0.010 \\
\hline CF/Assets ${ }_{t}$ & $\begin{array}{c}0.075 \\
(1.265)\end{array}$ & $\begin{array}{l}-0.036 \\
(2.102)\end{array}$ & 0.065 & 0.034 \\
\hline$C F /$ Assets $_{t+1}$ & $\begin{array}{c}0.131 \\
(1.250)\end{array}$ & $\begin{array}{c}0.038 \\
(1.265)\end{array}$ & 0.073 & 0.070 \\
\hline
\end{tabular}


Table 2. Panel B (continued)

$B E / M E_{t}$

NWC/Assets $t_{t}$

$\mathrm{ROA}_{t}$

$R O E_{t}$

$R O I_{t}$

Net Finance $_{t}$

INT Coverage $t_{t}$

Payout Ratio

Distribution to

Shareholder $t_{t}$

G-Index $t_{t}$

\begin{tabular}{|c|c|c|c|}
\hline \multicolumn{2}{|c|}{ Mean } & \multicolumn{2}{|c|}{ Median } \\
\hline $\begin{array}{c}\text { Positive } \\
\text { Residual cash }\end{array}$ & $\begin{array}{c}\text { Negative } \\
\text { residual cash }\end{array}$ & $\begin{array}{c}\text { Positive } \\
\text { Residual cash }\end{array}$ & $\begin{array}{c}\text { Negative } \\
\text { residual cash }\end{array}$ \\
\hline $\begin{array}{c}0.517 \\
(1.321)\end{array}$ & $\begin{array}{c}0.742 \\
(2.562)\end{array}$ & 0.458 & 0.614 \\
\hline $\begin{array}{c}0.034 \\
(0.816)\end{array}$ & $\begin{array}{c}0.032 \\
(2.912)\end{array}$ & 0.069 & 0.071 \\
\hline $\begin{array}{c}0.115 \\
(0.125)\end{array}$ & $\begin{array}{l}-0.085 \\
(1.205)\end{array}$ & 0.085 & 0.020 \\
\hline $\begin{array}{c}0.231 \\
(2.131)\end{array}$ & $\begin{array}{l}-0.021 \\
(2.897)\end{array}$ & 0.156 & 0.051 \\
\hline $\begin{array}{c}0.090 \\
(1.202)\end{array}$ & $\begin{array}{c}0.049 \\
(1.103)\end{array}$ & 0.088 & 0.034 \\
\hline $\begin{array}{c}0.159 \\
(0.773)\end{array}$ & $\begin{array}{c}0.063 \\
(0.539)\end{array}$ & 0.105 & 0.040 \\
\hline $\begin{array}{c}8.069 \\
(22.592)\end{array}$ & $\begin{array}{c}2.917 \\
(15.481)\end{array}$ & 3.857 & 2.160 \\
\hline $\begin{array}{c}0.140 \\
(2.508)\end{array}$ & $\begin{array}{c}0.260 \\
(5.695)\end{array}$ & 0.132 & 0 \\
\hline 0.026 & 0.020 & 0.022 & 0 \\
\hline $\begin{array}{c}(0.108) \\
8.225 \\
(2.589)\end{array}$ & $\begin{array}{c}(0.086) \\
9.180 \\
(2.734) \\
\end{array}$ & 8.000 & 9.000 \\
\hline
\end{tabular}




\section{Table 3. The Impact of Normal and Residual Cash on Firm Value}

This table shows the results of value regressions. The dependent variable is the market to book ratio. The independent variables include: 1 year lagged corporate liquidity (Corporate Liquidity $\left.\mathrm{t}_{\mathrm{t}-1}\right), 1$ year change in corporate liquidity $\left(\Delta\right.$ Corporate Liquidity $_{\mathrm{t}}$ ), the interaction between corporate liquidity and positive residual cash dummy (Corporate Liquidity $\mathrm{t}_{\mathrm{t}-1}$ Positive Dummy $\left.\mathrm{t}_{\mathrm{t}-1}\right), 1$ year lagged normal cash holding, calculated as the predicted value from regression (3) in Table 1 divided by total assets (Normal Cash/Assets), 1 year lagged residual cash holding, calculated as the residual from regression (3) in Table 1 divided by total assets (Residual Cash/Assets), the interaction term between residual cash and positive residual cash dummy (Residual Cash Holding ${ }_{t-1} *$ Positive Dummy $_{t}$ ), the absolute value of residual cash ( $\mid$ Residual Cash Holding $\left._{t-1} \mid\right)$, the squared absolute value of residualcash ( $\mid$ Residual Cash Holding $\left.\left.{ }_{\mathrm{t}-1}\right|^{2}\right), 1$ year change in net assets $(\Delta$ Net Assets $)$, current realizations and 1 year change in earnings (E), research and development expenses (R\&D), interest expenses (Interest) and dividend (Dividend) over assets, 1 year change of market to book ratio $\left(\Delta \mathrm{MKTtoBK}_{\mathrm{t}+1}\right)$, current leverage $\left(\right.$ Leverage $_{\mathrm{t}}$ ) and net finance $\left(\right.$ Net Finance $_{\mathrm{t}}$ ). All variables are winsorized at $1 \%$ and $99 \%$ tails. All models are estimated as fixed effects regressions and include year dummies. $t$-statistics are given in parentheses.

\begin{tabular}{|c|c|c|c|c|c|c|}
\hline & (1) & $(2)$ & (3) & $(4)$ & $(5)$ & $(6)$ \\
\hline Intercept & $\begin{array}{l}1.280 \\
(3.43)^{* * *}\end{array}$ & $\begin{array}{l}1.244 \\
(3.33)^{* * *}\end{array}$ & $\begin{array}{l}1.565 \\
(4.01)^{* * *}\end{array}$ & $\begin{array}{l}0.907 \\
(10.87)^{* * *}\end{array}$ & $\begin{array}{l}0.741 \\
(3.84)^{* * *}\end{array}$ & $\begin{array}{l}0.292 \\
(4.10)^{* * *}\end{array}$ \\
\hline Corporate Liquidity $_{t-1}$ & $\begin{array}{l}2.603 \\
(12.24)^{* * *}\end{array}$ & $\begin{array}{l}2.609 \\
(12.23)^{* * *}\end{array}$ & - & $\begin{array}{l}- \\
-\end{array}$ & - & - \\
\hline$\Delta$ Corporate Liquidityt $_{t}$ & $\begin{array}{l}-0.066 \\
(-5.14)^{* * *}\end{array}$ & - & - & - & - & - \\
\hline $\begin{array}{l}{\text { Corporate } \text { Liquidity }_{t-1}} \times \\
\text { Positive Dummy }_{t-1}\end{array}$ & - & $\begin{array}{l}2.630 \\
(2.12)^{* * *}\end{array}$ & - & - & - & - \\
\hline Normal Cash Holding ${ }_{t-1}$ & - & - & $\begin{array}{l}10.498 \\
(18.66)^{* * *}\end{array}$ & $\begin{array}{l}11.114 \\
(19.61)^{* * *}\end{array}$ & $\begin{array}{l}12.232 \\
(34.36)^{* * *}\end{array}$ & $\begin{array}{l}12.216 \\
(34.21)^{* * *}\end{array}$ \\
\hline $\mid$ Residual Cash Holding $_{t-1} \mid$ & - & - & $\begin{array}{l}-0.927 \\
(-1.96)^{* * *}\end{array}$ & $\begin{array}{l}-5.586 \\
(-5.10)\end{array}$ & $\begin{array}{l}-1.776 \\
(-6.32)\end{array}$ & $\begin{array}{l}-3.122 \\
(-7.04)\end{array}$ \\
\hline $\begin{array}{l}\mid \text { Residual Cash Holding }_{t-1} \mid \\
\times \text { Positive Dummy }_{t-1}\end{array}$ & - & - & - & - & - & 1.918 \\
\hline & - & - & - & - & - & $(4.91)^{* * *}$ \\
\hline$\triangle$ Residual Cash Holding $_{t}$ & $\begin{array}{l}- \\
-\end{array}$ & $\begin{array}{l}- \\
-\end{array}$ & $\begin{array}{l}- \\
-\end{array}$ & $\begin{array}{l}- \\
-\end{array}$ & $\begin{array}{l}-2.918 \\
(-9.53)\end{array}$ & $\begin{array}{l}-2.612 \\
(-8.80)\end{array}$ \\
\hline Positive Dummy $_{t-1}$ & $\begin{array}{l}- \\
-\end{array}$ & $\begin{array}{l}- \\
-\end{array}$ & $\begin{array}{l}- \\
-\end{array}$ & $\begin{array}{l}- \\
-\end{array}$ & $\begin{array}{l}0.161 \\
(5.48)^{* * *}\end{array}$ & - \\
\hline $\mid$ Residual Cash Holding $\left._{t-1}\right|^{2}$ & - & - & - & $\begin{array}{l}17.458^{* * *} \\
(5.82)^{2}\end{array}$ & - & - \\
\hline$\Delta M K T t o B K_{t+1}$ & $\begin{array}{l}0.002 \\
(5.51)^{* * *}\end{array}$ & $\begin{array}{l}0.002 \\
(5.96)^{* * *}\end{array}$ & $\begin{array}{l}-0.004^{* * *} \\
(2.67)^{2}\end{array}$ & $\begin{array}{l}0.0002 \\
(0.015)\end{array}$ & $\begin{array}{l}0.021 \\
(1.26)\end{array}$ & $\begin{array}{l}0.019 \\
(1.14)\end{array}$ \\
\hline$\Delta$ Net Assets $_{t}$ & $\begin{array}{l}-0.017 \\
(0.38)\end{array}$ & $\begin{array}{l}-0.021 \\
(-0.46)\end{array}$ & $\begin{array}{l}-0.042 \\
(-0.21)\end{array}$ & $\begin{array}{l}-0.034 \\
(-0.16)\end{array}$ & $\begin{array}{l}-0.492 \\
(-4.08)\end{array}$ & $\begin{array}{l}-0.493 \\
(-4.09)^{* * *}\end{array}$ \\
\hline$E_{t} /$ Assets $_{t}$ & $\begin{array}{l}-5.896 \\
(-47.31)^{* * *}\end{array}$ & $\begin{array}{l}-5.963 \\
(-48.01)^{* * *}\end{array}$ & $\begin{array}{l}11.519 \\
(19.12)^{* * *}\end{array}$ & $\begin{array}{l}11.286 \\
(18.58)^{* * *}\end{array}$ & $\begin{array}{l}10.469 \\
(34.38)^{* * *}\end{array}$ & $\begin{array}{l}10.535 \\
(34.62)^{* * *}\end{array}$ \\
\hline$\Delta E_{t} /$ Assets $_{t}$ & $\begin{array}{l}-0.070 \\
(-2.10)^{* * *}\end{array}$ & $\begin{array}{l}-0.072 \\
(-2.18)^{* * *}\end{array}$ & $\begin{array}{l}-5.581 \\
(-9.47)^{* * *}\end{array}$ & $\begin{array}{l}-7.038 \\
(-11.58)^{* * *}\end{array}$ & $\begin{array}{l}-3.641 \\
(-15.09)^{* * *}\end{array}$ & $\begin{array}{l}-3.651 \\
(-15.12)^{* * *}\end{array}$ \\
\hline$R \& D_{t} /$ Assets $_{t}$ & $\begin{array}{l}0.249 \\
(0.89)\end{array}$ & $\begin{array}{l}0.252 \\
(0.90)\end{array}$ & $\begin{array}{l}-1.817 \\
(-1.63)\end{array}$ & $\begin{array}{l}-2.479 \\
(-2.47)\end{array} * * *$ & $\begin{array}{l}-2.852 \\
(-4.71)^{* * *}\end{array}$ & $\begin{array}{l}-2.645 \\
(-4.38)\end{array}$ \\
\hline$\Delta R \& D_{t} /$ Assets $_{t}$ & $\begin{array}{l}0.144 \\
(4.90)\end{array}$ & $\begin{array}{l}-0.002 \\
(-0.23)\end{array}$ & $\begin{array}{l}-0.176 \\
(-1.56)\end{array}$ & $\begin{array}{l}0.573 \\
(21.38)^{* * *}\end{array}$ & $\begin{array}{l}4.066 \\
(3.99)^{* * *}\end{array}$ & $\begin{array}{l}4.008 \\
(3.93)^{* * *}\end{array}$ \\
\hline Interest $_{t} /$ Assets $_{t}$ & $\begin{array}{l}-9.113 \\
(-37.58)^{* * *}\end{array}$ & $\begin{array}{l}-9.019 \\
(-37.29)^{* * *}\end{array}$ & $\begin{array}{l}1.792 \\
(0.51)\end{array}$ & $\begin{array}{l}5.067 \\
(1.54)\end{array}$ & $\begin{array}{l}-15.511^{* * *} \\
(-8.26)^{4}\end{array}$ & $\begin{array}{l}-15.362^{* * *} \\
(-8.16)^{* *}\end{array}$ \\
\hline$\Delta$ Interest $_{t} /$ Assets $_{t}$ & $\begin{array}{l}0.234 \\
(7.87)^{* * *}\end{array}$ & $\begin{array}{l}0.099 \\
(7.07)^{* * *}\end{array}$ & $\begin{array}{l}0.232 \\
(12.35)^{* * *}\end{array}$ & $\begin{array}{l}0.314 \\
(23.27)^{* * *}\end{array}$ & $\begin{array}{l}1.798 \\
(0.81)\end{array}$ & $\begin{array}{l}1.709 \\
(0.77)\end{array}$ \\
\hline Dividend $_{t} /$ Assets $_{t}$ & $\begin{array}{l}5.303 \\
(7.24)^{* * *}\end{array}$ & $\begin{array}{l}5.252 \\
(7.17)^{* * *}\end{array}$ & $\begin{array}{l}2.944 \\
(4.87)^{* * *}\end{array}$ & $\begin{array}{l}3.128 \\
(5.06)\end{array}{ }^{* * *}$ & $\begin{array}{l}10.368 \\
(15.37)^{\text {*** }}\end{array}$ & $\begin{array}{l}10.371 \\
(15.36)^{* * *}\end{array}$ \\
\hline$\Delta$ Dividend $_{t} /$ Assets $_{t}$ & $\begin{array}{l}-0.209 \\
(-9.89)^{* * *}\end{array}$ & $\begin{array}{l}-0.144 \\
(-8.50)^{* * *}\end{array}$ & $\begin{array}{l}-0.563 \\
(-10.67)^{* * *}\end{array}$ & $\begin{array}{l}-0.840 \\
(-26.05)^{* * *}\end{array}$ & $\begin{array}{l}-1.730 \\
(-4.33)^{* * *}\end{array}$ & $\begin{array}{l}-1.735 \\
(-4.34)^{* * *}\end{array}$ \\
\hline Leverage $_{t}$ & $\begin{array}{l}1.280 \\
(11.05)^{* * *}\end{array}$ & $\begin{array}{l}1.260 \\
(10.87)^{* * *}\end{array}$ & $\begin{array}{l}-0.320 \\
(-0.88)\end{array}$ & $\begin{array}{l}-0.493 \\
(-1.43)\end{array}$ & $\begin{array}{l}2.509 \\
(14.67)^{* * *}\end{array}$ & $\begin{array}{l}2.493 \\
(14.56)^{* * *}\end{array}$ \\
\hline Net Finance $_{t}$ & $\begin{array}{l}1.968 \\
(13.38)^{* * *}\end{array}$ & $\begin{array}{l}1.955 \\
(13.29)^{* * *}\end{array}$ & $\begin{array}{l}0.854 \\
(2.78)^{* * *}\end{array}$ & $\begin{array}{l}1.122 \\
(3.66)^{* * *}\end{array}$ & $\begin{array}{l}-1.248 \\
(-6.41)^{* * *}\end{array}$ & $\begin{array}{l}-1.216 \\
(-6.24)^{* * *}\end{array}$ \\
\hline Adjusted $R^{2}$ & 0.25 & 0.25 & 0.89 & 0.88 & 0.86 & 0.86 \\
\hline
\end{tabular}




\section{Table 4. Bankruptcies and Failures as Percentage of Active Firms}

This table presents the number of bankruptcies and failures, total number of active firms and the percentage of bankruptcies each year during the sample period of 1950 - 2005. The bankruptcy sample includes all bankruptcy filings as reported in the COMPUSTAT and CRSP datasets. Firms with a CRSP bankruptcy delisting code 400-490 (liquidations), 572 (liquidations) and 574 (bankruptcy), and firms with COMPUSTAT footnote 35 having code 02 (Chapter 11) or 03 (Chapter 7) are considered as failing and will be included in the bankruptcy sub sample. There are a total of 874 bankruptcies in the sample.

\begin{tabular}{cccccccc}
\hline & $\begin{array}{c}\text { \# of } \\
\text { Bankrupt }\end{array}$ & $\begin{array}{c}\text { \# of } \\
\text { Active }\end{array}$ & $\begin{array}{c}\text { \% of Bankrupt } \\
\text { to Active }\end{array}$ & & $\begin{array}{c}\text { \# of } \\
\text { Bankrupt }\end{array}$ & $\begin{array}{c}\text { \# of } \\
\text { Active }\end{array}$ & $\begin{array}{c}\text { \% of Bankrupt } \\
\text { to Active }\end{array}$ \\
Year & Firms & Firms & Firms & Year & Firms & Firms & Firms \\
\hline 1950 & 0 & 425 & $0.00 \%$ & 1978 & 22 & 4223 & $0.52 \%$ \\
1951 & 0 & 440 & $0.00 \%$ & 1979 & 21 & 4187 & $0.50 \%$ \\
1952 & 0 & 447 & $0.00 \%$ & 1980 & 33 & 4296 & $0.77 \%$ \\
1953 & 0 & 453 & $0.00 \%$ & 1981 & 21 & 4562 & $0.46 \%$ \\
1954 & 0 & 466 & $0.00 \%$ & 1982 & 31 & 4619 & $0.67 \%$ \\
1955 & 0 & 474 & $0.00 \%$ & 1983 & 26 & 5099 & $0.51 \%$ \\
1956 & 0 & 486 & $0.00 \%$ & 1984 & 25 & 5293 & $0.47 \%$ \\
1957 & 0 & 511 & $0.00 \%$ & 1985 & 25 & 5347 & $0.47 \%$ \\
1958 & 0 & 521 & $0.00 \%$ & 1986 & 53 & 5663 & $0.94 \%$ \\
1959 & 0 & 543 & $0.00 \%$ & 1987 & 18 & 5793 & $0.31 \%$ \\
1960 & 0 & 573 & $0.00 \%$ & 1988 & 40 & 5770 & $0.69 \%$ \\
1961 & 0 & 604 & $0.00 \%$ & 1989 & 39 & 5573 & $0.70 \%$ \\
1962 & 0 & 1070 & $0.00 \%$ & 1990 & 33 & 5412 & $0.61 \%$ \\
1963 & 0 & 1136 & $0.00 \%$ & 1991 & 61 & 5430 & $1.12 \%$ \\
1964 & 0 & 1211 & $0.00 \%$ & 1992 & 34 & 5620 & $0.60 \%$ \\
1965 & 0 & 1290 & $0.00 \%$ & 1993 & 19 & 6100 & $0.31 \%$ \\
1966 & 1 & 1365 & $0.07 \%$ & 1994 & 6 & 6429 & $0.09 \%$ \\
1967 & 0 & 1555 & $0.00 \%$ & 1995 & 17 & 6603 & $0.26 \%$ \\
1968 & 1 & 1700 & $0.06 \%$ & 1996 & 13 & 6932 & $0.19 \%$ \\
1969 & 1 & 1861 & $0.05 \%$ & 1997 & 16 & 6975 & $0.23 \%$ \\
1970 & 4 & 1985 & $0.20 \%$ & 1998 & 25 & 6835 & $0.37 \%$ \\
1971 & 6 & 1965 & $0.31 \%$ & 1999 & 22 & 6567 & $0.34 \%$ \\
1972 & 5 & 4215 & $0.12 \%$ & 2000 & 33 & 6268 & $0.53 \%$ \\
1973 & 12 & 4328 & $0.28 \%$ & 2001 & 45 & 5788 & $0.78 \%$ \\
1974 & 13 & 4211 & $0.31 \%$ & 2002 & 43 & 5297 & $0.81 \%$ \\
1975 & 14 & 4172 & $0.34 \%$ & 2003 & 25 & 5023 & $0.50 \%$ \\
1976 & 15 & 4221 & $0.36 \%$ & 2004 & 19 & 4852 & $0.39 \%$ \\
1977 & 18 & 4217 & $0.43 \%$ & 2005 & 19 & 4739 & $0.40 \%$ \\
\hline
\end{tabular}




\section{Table 5. Logistic Regression of on Firm Characteristics as Bankruptcy Predictors}

This table reports results from logit regressions of the bankruptcy and failure indicator on firm characteristics that can serve as predictor variables. The value of the predictors is known at the beginning of the period during which bankruptcy or failure occurs.

Chi-square values are in parenthesis. The chi-square of the likelihood ratio test for the model fit is reported in the model fit row.

\section{(1)}

\begin{tabular}{|c|c|c|c|c|}
\hline (1) & (2) & (3) & (4) & (5) \\
\hline $\begin{array}{l}7.24 \\
(2207.25)^{* * *}\end{array}$ & $\begin{array}{l}6.50 \\
(719.53)^{* * *}\end{array}$ & $\begin{array}{l}5.24 \\
(366.06)^{* * *}\end{array}$ & $\begin{array}{l}5.41 \\
(390.24)^{* * *}\end{array}$ & $\begin{array}{l}5.37 \\
(377.43)^{* * *}\end{array}$ \\
\hline-0.22 & -0.41 & -0.20 & -0.32 & -0.29 \\
\hline$(10.19)^{* * *}$ & $(14.11)^{* * *}$ & $(6.34)^{* * *}$ & $(9.28)^{* * * *}$ & $(8.34)^{* * *}$ \\
\hline $\begin{array}{l}0.38 \\
(13.78)^{* * *}\end{array}$ & $\begin{array}{l}1.26 \\
(62.77)^{* * *}\end{array}$ & $\begin{array}{l}0.44 \\
(10.58)^{* * *}\end{array}$ & $\begin{array}{l}0.64 \\
(10.34)^{* * *}\end{array}$ & $\begin{array}{l}0.56 \\
(9.34)^{* * *}\end{array}$ \\
\hline-0.91 & -1.13 & -0.63 & -0.71 & -0.79 \\
\hline$(29.22)^{* . * 4}$ & $(28.22)^{* * *}$ & $(17.62)^{* * * 4}$ & $(20.34)^{* 3 * 4}$ & $(19.89)^{* * * 4}$ \\
\hline $\begin{array}{l}2.07 \\
(30.84)^{* * *}\end{array}$ & $\begin{array}{l}2.30 \\
(31.98)^{* * * *}\end{array}$ & $\begin{array}{l}1.17 \\
(8.59)^{* * *}\end{array}$ & $\begin{array}{l}1.65 \\
(18.45)^{* * *}\end{array}$ & $\begin{array}{l}1.89 \\
(20.34)^{* * *}\end{array}$ \\
\hline $\begin{array}{l}-0.36 \\
(72.22)^{* * *}\end{array}$ & $\begin{array}{l}-0.24 \\
(23.68)^{* * *}\end{array}$ & $\begin{array}{l}-0.09 \\
(3.02)^{*}\end{array}$ & $\begin{array}{l}-0.08 \\
(8.87)^{* * *}\end{array}$ & $\begin{array}{l}-0.14 \\
(9.84)^{* * *}\end{array}$ \\
\hline- & 0.13 & 0.07 & 0.07 & 0.06 \\
\hline- & $(5.16)^{* *}$ & $(0.94)$ & $(2.32)$ & (2.39) \\
\hline - & -0.44 & -0.58 & -0.45 & -0.48 \\
\hline- & $(20.06)^{* * *}$ & $(59.69)^{* * *}$ & $(34.43)^{* * *}$ & $(24.36)^{* * *}$ \\
\hline - & - & -1.16 & - & - \\
\hline - & - & $(6.64)^{* * *}$ & - & - \\
\hline - & - & - & -0.86 & -0.77 \\
\hline - & - & - & $(-2.56)$ & $(-2.14)$ \\
\hline - & - & - & - & - \\
\hline - & - & - & - & - \\
\hline - & - & - & 1.05 & 1.12 \\
\hline- & - & - & $(6.12)^{* * *}$ & $(6.34)^{* * *}$ \\
\hline- & - & - & - & 0.45 \\
\hline - & - & - & - & $(5.48)^{* * *}$ \\
\hline - & - & - & -0.63 & -0.56 \\
\hline - & - & - & $(5.34)^{* * * *}$ & $(5.04)^{* * *}$ \\
\hline - & - & . & 0.35 & - \\
\hline - & - & $\begin{array}{l}- \\
-\end{array}$ & $(5.09)^{* *}$ & - \\
\hline 0.09 & 0.12 & 0.14 & 0.18 & 0.17 \\
\hline $216.56^{* * *}$ & $416.03^{* * *}$ & $405.12^{* * *}$ & $446.43^{* * *}$ & $436.54^{* * *}$ \\
\hline 741 & 721 & 712 & 689 & 689 \\
\hline 94695 & 93859 & 90874 & 93854 & 93854 \\
\hline
\end{tabular}




\section{Table 6. Regression of Default Probability Measures on Firm Characteristics}

This table presents the results of various regressions of firm characteristics on different default probability measures. The independent variables include: Altman's Z-score, Vassalou and Xing's Default Likelihood Indicator (DLI) and Chava and Jarrow's bankruptcy probability. Altman' Z-score is a bankruptcy predictor that determines the likelihood of a firm going bankrupt by a multivariate formula combining five common business ratios. Z-score is calculated as follows: $\mathrm{Z}=.012 \mathrm{X}_{1}+.014 \mathrm{X}_{2}+.033 \mathrm{X}_{3}+.006 \mathrm{X}_{4}+.999 \mathrm{X}_{5}$, where $\mathrm{X}_{1}=$ Working capital/Total assets, $\mathrm{X}_{2}=$ Retained earnings Total assets, $\mathrm{X}_{3}=$ Earnings before interest and taxes/Total assets, $\mathrm{X}_{4}=$ Market value equity/Book value of total debt, $\mathrm{X}_{5}=$ Sales/Total assets, and Z=Over all index. Vassalou and Xing (2004)'s Default Likelihood Indicator (DLI) is calculated based on Black and Scholes (1973) contingent claims methodology and Merton's (1974) option pricing model using equity data. The bankruptcy probability is constructed by Chava and Jarrow (2004). They use the bankruptcy hazard rate model and an expanded bankruptcy database to estimate the probability of bankruptcy and failure. The regressions cover the sample period where the above default probabilities are available. $t$-statistics are in parenthesis.

Panel A: Dependent Variable - Z-score

(2)

(3)

(4)

(5)

(6)

Constant

$N I / T A_{t-1}$

Leverage $_{t-1}$

ExRet $_{t-1}$

$\operatorname{SIGMA}_{t-1}$

$\operatorname{RSIZE}_{t-1}$

$M k t T o B k_{t-1}$

PRICE $_{t-1}$

$C_{-} T A_{t-1}$

$C_{-} T A_{t-1} \times$ Negative

Dummy $_{t-1}$

Normal Cash Holding $_{t-1}$

|Residual Cash Holding ${ }_{t-1} \mid$

$\mid$ Residual Cash Holding ${ }_{t-1} \mid$

$\times$ Negative Dummy $_{t-1}$

Negative Dummy ${ }_{t-1}$

$\begin{array}{ll}1.53 & 1.43 \\ (30.43)^{* * *} & (28.21)^{* * *}\end{array}$

0.36

0.15

$(10.84)^{* * *}$

$-0.20$

$(12.06)^{* * *}$

$-0.10$

$(-6.04)^{* * *}$

$(-4.39)^{* * *}$

0.05

0.05

$(5.50)^{* * *}$

$(8.15)^{* * *}$

$-0.86$

$-0.97$

$(-10.18)^{* * *}$

0.08

$(-16.02)^{* * *}$

1.62

$(62.36)^{* * *}$

0.11
$(8.54)^{* * *}$

$-0.54$

$(-21.65)^{* * *}$

0.04

$(7.25)^{* * *}$

$-0.57$

0.07

$(-9.46)^{* * *}$

0.07

$(21.29)^{* * *}$

$(17.39)^{* * *}$

$(19.33)^{* * *}$

$-0.01$

$-0.02$

$(-2.84)^{* * *}$

$(-6.62)^{* * *}$

$(-2.84)$
0.02

0.02

$(3.04)^{* * *}$

- $\quad(3.27)^{* * *}$

1.37

$\begin{array}{lll}- & - & \\ - & - & (32.28)^{* * *}\end{array}$

1.53
$(31.71)^{* * *}$
0.11
$(8.88)^{* * *}$
-0.54
$(-21.30)^{* * *}$
0.04

1.07

$(25.35)^{* * *}$

1.01

0.39

$(3.02)^{* * *}$

$(22.21)^{* * * *}$

$-0.60$

0.40
$(3.13)^{* * *}$

$(-8.58)^{* * *}$

$-0.59$

$0.04 \quad 0.05$

$(-8.50)^{* * *}$

$(6.48)^{* * *}$

$(2.79)^{* * *}$

0.05

$-0.59$

$-0.25$

$(2.82)^{* * *}$

$(-9.70)^{* * *}$

$(-1.18)$

0.07

$$
0.05
$$

$(19.34)^{* * *}$

$(5.55)^{* * *}$

$-0.22$

$(-1.03)$

$-0.01$

$-0.03$

0.05

$(-4.94)^{* * *}$

$(-7.11)^{* * *}$

$(5.15)^{* * *}$

$-0.08$

0.02

$(3.00)^{* * *}$

0.01

$(-8.43)^{* * *}$

0.04

1.98

$(7.44)^{* * *}$

$(-2.18)$

$(3.28)^{* * *}$

$-0.44$

-

$(-2.25)^{* *}$

$-2.08$

$(-9.99)^{* * * *}$

$-2.21$

$-0.17$

$(-10.44)^{* * *}$

$(-0.85)$

$-0.83$

$(-2.54)^{* *}$

-

$-$

$-\quad-1.20$

$-$

- $\quad-$

- $\quad-\quad(-4.20)^{* * *}$

Negative Dummy

$\begin{array}{ll}R^{2} & 0.05\end{array}$

0.07

0.09

0.11

0.12

0.15 
Table 6. (continued)

Panel B: Dependent Variable - DLI

(1)

(2)

(3)

(4)

(5)

(6)

\begin{tabular}{|c|c|c|c|c|c|c|}
\hline Constant & $\begin{array}{l}-0.05 \\
(-42.59)^{* * *}\end{array}$ & $\begin{array}{l}0.02 \\
(4.88)^{* * *}\end{array}$ & $\begin{array}{l}0.02 \\
(5.44)^{* * *}\end{array}$ & $\begin{array}{l}-0.03 \\
(-2.54)^{* * *}\end{array}$ & $\begin{array}{l}0.05 \\
(3.50)^{* * *}\end{array}$ & $\begin{array}{l}0.00 \\
(0.44)\end{array}$ \\
\hline$N I / T A_{t-I}$ & $\begin{array}{l}-0.03 \\
(-13.16)^{* * *}\end{array}$ & $\begin{array}{l}-0.07 \\
(-14.29)^{* * *}\end{array}$ & $\begin{array}{l}-0.03 \\
(-12.42)^{* * *}\end{array}$ & $\begin{array}{l}-0.08 \\
(-16.54)^{* * *}\end{array}$ & $\begin{array}{l}-0.09 \\
(-3.59)^{* * *}\end{array}$ & $\begin{array}{l}-0.09 \\
(-3.37)^{* * *}\end{array}$ \\
\hline Leverage $_{t-1}$ & $\begin{array}{l}0.22 \\
(84.13)^{* * *}\end{array}$ & $\begin{array}{l}0.20 \\
(55.66)^{* * *}\end{array}$ & $\begin{array}{l}0.22 \\
(70.65)^{* * *}\end{array}$ & $\begin{array}{l}0.22 \\
(57.60)^{* * *}\end{array}$ & $\begin{array}{l}0.03 \\
(2.65)^{* * *}\end{array}$ & $\begin{array}{l}0.04 \\
(3.25)^{* * *}\end{array}$ \\
\hline $\operatorname{ExRet}_{t-1}$ & $\begin{array}{l}-0.04 \\
(-53.26)^{* * *}\end{array}$ & $\begin{array}{l}-0.04 \\
(-32.00)^{* * *}\end{array}$ & $\begin{array}{l}-0.03 \\
(-37.16)^{* * *}\end{array}$ & $\begin{array}{l}-0.05 \\
(-42.42)^{* * *}\end{array}$ & $\begin{array}{l}-0.02 \\
(-5.39)^{* * *}\end{array}$ & $\begin{array}{l}-0.02 \\
(-6.23)^{* * *}\end{array}$ \\
\hline$S I G M A_{t-1}$ & $\begin{array}{l}0.20 \\
(29.68)^{* * *}\end{array}$ & $\begin{array}{l}0.22 \\
(19.16)^{* * *}\end{array}$ & $\begin{array}{l}0.17 \\
(19.98)^{* * *}\end{array}$ & $\begin{array}{l}0.33 \\
(29.64)^{* * *}\end{array}$ & $\begin{array}{l}0.04 \\
(0.89)\end{array}$ & $\begin{array}{l}0.07 \\
(1.69)^{*}\end{array}$ \\
\hline$R S I Z E_{t-1}$ & $\begin{array}{l}-0.01 \\
(-47.72)^{* * *}\end{array}$ & $\begin{array}{l}-0.01 \\
(-14.16)^{* * *}\end{array}$ & $\begin{array}{l}-0.01 \\
(-14.04)^{* * *}\end{array}$ & $\begin{array}{l}-0.02 \\
(-37.99)^{* * *}\end{array}$ & $\begin{array}{l}-0.01 \\
(-10.30)^{* * *}\end{array}$ & $\begin{array}{l}-0.01 \\
(-4.20)^{* * *}\end{array}$ \\
\hline$M k t T o B k_{t-1}$ & - & $\begin{array}{l}0.01 \\
(4.22)^{* * *}\end{array}$ & $\begin{array}{l}0.01 \\
(2.00)^{* * *}\end{array}$ & $\begin{array}{l}0.01 \\
(3.02)^{* * *}\end{array}$ & $\begin{array}{l}0.01 \\
(2.12)^{* *}\end{array}$ & $\begin{array}{l}0.00 \\
(0.94)\end{array}$ \\
\hline$P R I C E_{t-l}$ & - & $\begin{array}{l}-0.02 \\
(-19.85)^{* * *}\end{array}$ & $\begin{array}{l}-0.02 \\
(-23.37)^{* * *}\end{array}$ & $\begin{array}{l}-0.01 \\
(24.48)^{* * *}\end{array}$ & $\begin{array}{l}-0.01 \\
(-3.40)^{* * *}\end{array}$ & $\begin{array}{l}-0.01 \\
(-2.14)^{* *}\end{array}$ \\
\hline$C_{-} T A_{t-1}$ & - & - & $\begin{array}{l}0.01 \\
(1.42)\end{array}$ & $\begin{array}{l}0.002 \\
(1.18)\end{array}$ & - & - \\
\hline $\begin{array}{l}\text { C_TA } A_{t-1} \times \text { Negative } \\
\text { Dummy } \\
t-1\end{array}$ & - & - & - & 0.01 & - & - \\
\hline & - & - & - & $(2.82)^{* * *}$ & - & - \\
\hline Normal Cash Holding $_{t-1}$ & - & - & - & - & $\begin{array}{l}0.01 \\
(0.31)\end{array}$ & $\begin{array}{l}0.02 \\
(0.43)\end{array}$ \\
\hline $\mid$ Residual Cash Holding ${ }_{t-1} \mid$ & - & - & - & - & $\begin{array}{l}0.09 \\
(2.47)^{* *}\end{array}$ & $\begin{array}{l}0.18 \\
(3.04)^{* * *}\end{array}$ \\
\hline $\begin{array}{l}\mid \text { Residual Cash Holding } \text { H-1 } \mid \\
\times \text { Negative Dummy }_{t-1}\end{array}$ & - & - & - & - & - & 0.12 \\
\hline & - & - & - & - & - & $(2.28)^{* *}$ \\
\hline Negative Dummy $_{t-1}$ & - & - & - & - & $\begin{array}{l}0.01 \\
(1.99)^{* *}\end{array}$ & - \\
\hline$R^{2}$ & 0.25 & 0.29 & 0.30 & 0.31 & 0.33 & 0.34 \\
\hline
\end{tabular}


Table 6. (continued)

Panel C: Independent Variable - Default Probability

\begin{tabular}{|c|c|c|c|c|c|c|}
\hline 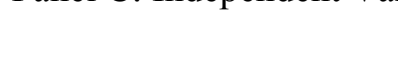 & $(1)$ & $(2)$ & (3) & (4) & $(5)$ & (6) \\
\hline Constant & $\begin{array}{l}0.001 \\
(2.80)^{* * *}\end{array}$ & $\begin{array}{l}0.004 \\
(7.55)^{* * *}\end{array}$ & $\begin{array}{l}-0.001 \\
(-1.13)\end{array}$ & $\begin{array}{l}0.004 \\
(6.85)^{* * *}\end{array}$ & $\begin{array}{l}-0.001 \\
(-0.50)\end{array}$ & $\begin{array}{l}-0.003 \\
(-1.43)\end{array}$ \\
\hline$N I / T A_{t-1}$ & $\begin{array}{l}-0.001 \\
(-0.98)\end{array}$ & $\begin{array}{l}-0.001 \\
(-0.76)\end{array}$ & $\begin{array}{l}-0.002 \\
(-1.90)\end{array}$ & $\begin{array}{l}-0.001 \\
(-0.86)\end{array}$ & $\begin{array}{l}-0.003 \\
(-2.09)^{* *}\end{array}$ & $\begin{array}{l}-0.002 \\
(-1.29)\end{array}$ \\
\hline Leverage $_{t-1}$ & $\begin{array}{l}0.002 \\
(4.28)^{* * *}\end{array}$ & $\begin{array}{l}0.001 \\
(2.70)^{* * *}\end{array}$ & $\begin{array}{l}0.002 \\
(4.79)^{* * *}\end{array}$ & $\begin{array}{l}0.002 \\
(3.57)^{* * *}\end{array}$ & $\begin{array}{l}0.004 \\
(6.24)^{* * *}\end{array}$ & $\begin{array}{l}0.002 \\
(5.90)^{* * *}\end{array}$ \\
\hline ExRet ${ }_{t-1}$ & $\begin{array}{l}-0.01 \\
(-1.87)^{*}\end{array}$ & $\begin{array}{l}0.001 \\
(0.75)\end{array}$ & $\begin{array}{l}-0.001 \\
(-2.25)^{* * *}\end{array}$ & $\begin{array}{l}0.001 \\
(0.56)\end{array}$ & $\begin{array}{l}0.001 \\
(2.91)^{* * *}\end{array}$ & $\begin{array}{l}0.001 \\
(2.78)^{* * *}\end{array}$ \\
\hline$S I G M A_{t-1}$ & $\begin{array}{l}0.01 \\
(7.77)^{* * *}\end{array}$ & $\begin{array}{l}0.01 \\
(4.72)^{* * *}\end{array}$ & $\begin{array}{l}0.01 \\
(7.24)^{* * *}\end{array}$ & $\begin{array}{l}0.01 \\
(4.17)^{* * *}\end{array}$ & $\begin{array}{l}0.01 \\
(4.53)^{* * *}\end{array}$ & $\begin{array}{l}0.01 \\
(4.45)^{* * *}\end{array}$ \\
\hline$R S I Z E_{t-1}$ & $\begin{array}{l}-0.001 \\
(-14.74)^{* * *}\end{array}$ & $\begin{array}{l}-0.001 \\
(-7.03)^{* * *}\end{array}$ & $\begin{array}{l}-0.001 \\
(-12.81)^{* * *}\end{array}$ & $\begin{array}{l}-0.004 \\
(-6.59)^{* * *}\end{array}$ & $\begin{array}{l}-0.003 \\
(-3.23)^{* * *}\end{array}$ & $\begin{array}{l}-0.003 \\
(-7.54)^{* * *}\end{array}$ \\
\hline$M k t T o B k_{t-1}$ & - & $\begin{array}{l}0.001 \\
(2.50)^{* * *}\end{array}$ & $\begin{array}{l}0.001 \\
(2.28)^{* * *}\end{array}$ & $\begin{array}{l}0.001 \\
(2.33)^{* * *}\end{array}$ & $\begin{array}{l}-0.001 \\
(-0.99)\end{array}$ & $\begin{array}{l}0.001 \\
(1.77)\end{array}$ \\
\hline$P R I C E_{t-1}$ & $\begin{array}{l}- \\
-\end{array}$ & $\begin{array}{l}-0.001 \\
(-7.80)^{* * *}\end{array}$ & $\begin{array}{l}0.000 \\
(1.27)\end{array}$ & $\begin{array}{l}-0.001 \\
(-7.59)^{* * *}\end{array}$ & $\begin{array}{l}-0.001 \\
(-2.10)^{* * *}\end{array}$ & $\begin{array}{l}-0.001 \\
(-1.95)\end{array}$ \\
\hline$C_{-} T A_{t-1}$ & - & - & $\begin{array}{l}0.004 \\
(4.31)^{* * *}\end{array}$ & $\begin{array}{l}0.001 \\
(0.79)\end{array}$ & $\begin{array}{l}- \\
-\end{array}$ & - \\
\hline $\begin{array}{l}\text { C_TA } A_{t-1} \times \text { Negative } \\
\text { Dummy }_{t-1}\end{array}$ & - & - & - & $\begin{array}{l}0.004 \\
(2.45)^{* *}\end{array}$ & - & - \\
\hline Normal Cash Holding $_{t-1}$ & $\begin{array}{l}- \\
-\end{array}$ & $\begin{array}{l}- \\
-\end{array}$ & - & - & $\begin{array}{l}0.002 \\
(0.79)\end{array}$ & $\begin{array}{l}-0.01 \\
(-1.52)\end{array}$ \\
\hline $\mid$ Residual Cash Holding ${ }_{t-1} \mid$ & $\begin{array}{l}- \\
-\end{array}$ & $\begin{array}{l}- \\
-\end{array}$ & $\begin{array}{l}- \\
-\end{array}$ & $\begin{array}{l}- \\
-\end{array}$ & $\begin{array}{l}0.01 \\
(2.78)^{* * *}\end{array}$ & $\begin{array}{l}0.01 \\
(2.96)^{* * *}\end{array}$ \\
\hline 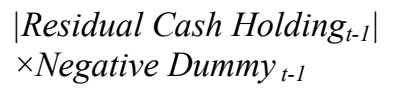 & - & - & - & - & - & $\begin{array}{l}0.01 \\
(2.37)^{* *}\end{array}$ \\
\hline Negative Dummy $_{t-1}$ & $\begin{array}{l}- \\
-\end{array}$ & - & - & $\begin{array}{l}- \\
-\end{array}$ & $\begin{array}{l}0.01 \\
(1.87)^{*}\end{array}$ & - \\
\hline$R^{2}$ & 0.19 & 0.20 & 0.21 & 0.22 & 0.23 & 0.25 \\
\hline
\end{tabular}




\section{Table 7. Forecast Regression of Residual Cash}

This table shows the result of the forecasting regression of residual cash can be estimated using the following two regressions for various holding horizons:

$$
X_{t, t+h}-\bar{X}_{t, t+h}=\alpha+\beta_{I} \mid \text { ResidualCash } \mid+\beta_{2} \text { Negative Dummy } y_{t}+\varepsilon_{t}
$$

$$
X_{t, t+h}-\bar{X}_{t, t+h}=\alpha+\beta_{1} \mid \text { Residual Cash }\left|+\beta_{2}\right| \text { Residual Cash } \mid \times \text { Negative Dummy } y_{t}+\varepsilon_{t},
$$

where $X_{t, t+h}$ is the variable to be predicted over the holding period from $t$ to $t+h$, and $\bar{X}_{t, t+h}$ is the benchmark of $X_{t, t+h} . X_{t, t+h}$ to be forecasted here includes: $R_{t, t+h}$, cumulative stock returns over the holding period from $t$ to $t+h ; R O A_{t, t+h}$, average return on assets over the period $t$ to $t+h$; Residual cas $h_{t, t+h}$, average residual cash different from the normal cash holding level estimated by equation (1) during period $t$ to $t+h ; R \& D_{t, t+h}$, average research and development expenditure in period $t$ to $t+h ; C F_{t, t+h}$, average cash flows during period $t$ to $t+h$; and Capital Expense $e_{t+t}$, average capital investment over period $t$ to $t+h$. Benchmark value $\bar{X}_{t, t+h}$ is measured as the industry-size average of $X_{t, t+h}$. First, in each period, all the firms are categorized into industry portfolios according to SIC code; then within each industry portfolio, firms are subsequently sorted into 5 size sub portfolios. Next, the industry-size norm of the variable $X_{t, t+h}$ is calculated each period by taking the average of $X$ of all firms falling into a certain industry-size portfolio over the period $t$ to $t+h$. $t$-statistics are given in parentheses.

\begin{tabular}{|c|c|c|c|c|c|c|c|}
\hline & & $R E T$ & $R O A$ & Residual Cash & $R \& D$ & Cash Flow & $\begin{array}{l}\text { Capital Expense } \\
\text { /Assets }\end{array}$ \\
\hline \multirow[t]{5}{*}{$X_{t, t+1}-\bar{X}_{t, t+1}$} & $\mid$ Residual Cash Holding $_{t} \mid$ & -0.45 & -0.01 & 0.33 & 0.07 & -0.08 & -0.02 \\
\hline & & $(-2.85)^{* * *}$ & $(-0.58)$ & $(19.99)^{* * *}$ & $(9.52)^{* * *}$ & $(-5.46)^{* * *}$ & $(-2.39)^{* *}$ \\
\hline & Negative Dummy $_{t}$ & -0.02 & -0.004 & -0.07 & -0.002 & -0.01 & -0.01 \\
\hline & & $(-0.75)$ & $(-2.33)^{* *}$ & $(-35.88)^{* * *}$ & $(-1.80)^{*}$ & $(-3.63)^{* * *}$ & $(-5.64)^{* * *}$ \\
\hline & $R^{2}$ & 0.05 & 0.01 & 0.30 & 0.06 & 0.03 & 0.02 \\
\hline \multirow[t]{5}{*}{$X_{t, t+2}-\bar{X}_{, t t+2}$} & $\mid$ Residual Cash Holding $_{t} \mid$ & -0.51 & -0.003 & 0.28 & 0.07 & -0.09 & -0.02 \\
\hline & & $(-3.75)^{* * *}$ & $(-0.21)$ & $(15.52)^{* * *}$ & $(7.53)^{* * *}$ & $(-5.12)^{* * *}$ & $(-2.29)^{* *}$ \\
\hline & Negative Dummy ${ }_{t}$ & -0.03 & -0.002 & -0.07 & -0.001 & -0.003 & -0.01 \\
\hline & & $(-1.45)$ & $(-0.90)$ & $(-31.78)^{* * *}$ & $(-1.35)$ & $(-1.41)$ & $(-6.27)^{* * *}$ \\
\hline & $R^{2}$ & 0.04 & 0.01 & 0.26 & 0.05 & 0.02 & 0.03 \\
\hline \multirow[t]{5}{*}{$X_{t, t+3}-\bar{X}_{t, t+3}$} & $\mid$ Residual Cash Holding $_{t} \mid$ & -0.37 & -0.01 & 0.21 & 0.05 & -0.10 & -0.02 \\
\hline & & $(-2.92)^{* * *}$ & $(-0.52)$ & $(10.94)^{* * *}$ & $(5.19)^{* * *}$ & $(-5.10)^{* * *}$ & $(-1.63)$ \\
\hline & Negative Dummy ${ }_{t}$ & -0.04 & -0.002 & -0.06 & -0.002 & -0.004 & -0.01 \\
\hline & & $(-2.23)^{* *}$ & $(-0.84)$ & $(-27.33)^{* * *}$ & $(-1.61)$ & $(-1.77)^{*}$ & $(-6.23)^{* * *}$ \\
\hline & $R^{2}$ & 0.03 & 0.01 & 0.21 & 0.03 & 0.02 & 0.03 \\
\hline
\end{tabular}

Panel A. $X_{t, t+h}-\bar{X}_{t, t+h}=\alpha+\beta_{1} \mid$ Residual Cash ${ }_{t} \mid+\beta_{2}$ Negative Dummy $_{t}+\varepsilon_{t}$ 
Table 7. (continued)

Panel B. $X_{t+h}-\bar{X}_{t+h}=\alpha+\beta_{1} \mid$ Residual Cash $t\left|+\beta_{2}\right|$ Residual Cash $_{t} \mid \times$ Negative Dummy $_{t}+\varepsilon_{t}$

\begin{tabular}{|c|c|c|c|c|c|c|c|}
\hline & & $R E T$ & $R O A$ & Residual Cash & $R \& D$ & Cash Flow & $\begin{array}{c}\text { Capital Expense } \\
\text { /Assets }\end{array}$ \\
\hline \multirow[t]{5}{*}{$X_{t, t+1}-\bar{X}_{t, t+1}$} & $\mid$ Residual Cash Holding $_{t} \mid$ & -0.90 & -0.01 & -0.62 & & -0.02 & -0.06 \\
\hline & & $(-3.42)^{* * *}$ & $(-0.23)$ & $(-27.26)^{* * *}$ & $(4.63)^{* * *}$ & $(-0.93)$ & $(-3.46)^{* * *}$ \\
\hline & $\begin{array}{l}\mid \text { Residual Cash Holding }_{t} \mid \times \\
\text { Negative Dummy }\end{array}$ & -0.58 & -0.004 & -1.34 & -0.02 & -0.06 & -0.05 \\
\hline & & $(-2.21)^{* *}$ & $(-0.15)$ & $(-58.38)^{* * *}$ & $(-1.62)$ & $(-2.49)^{* *}$ & $(-3.23)^{* * *}$ \\
\hline & $R^{2}$ & 0.03 & 0.01 & 0.49 & 0.06 & 0.03 & 0.02 \\
\hline \multirow[t]{5}{*}{$X_{t, t+2}-\bar{X}_{, t t+2}$} & $\mid$ Residual Cash Holding $_{t} \mid$ & -1.13 & 0.02 & -0.52 & 0.05 & -0.01 & -0.06 \\
\hline & & $(-5.13)^{* * *}$ & $(0.78)$ & $(-21.81)^{* * *}$ & $(3.29)^{* * *}$ & $(-0.28)$ & $(-3.58)^{* * *}$ \\
\hline & $\begin{array}{l}\mid \text { Residual Cash Holding }_{t} \mid \times \\
\text { Negative Dummy }\end{array}$ & -0.82 & 0.03 & -1.19 & -0.03 & -0.09 & -0.06 \\
\hline & & $(-3.73)^{* * *}$ & $(1.03)$ & $(-48.60)^{* * *}$ & $(-2.00)^{* *}$ & $(-3.42)^{* * *}$ & $(-3.53)^{* * *}$ \\
\hline & $R^{2}$ & 0.04 & 0.01 & 0.42 & 0.04 & 0.03 & 0.02 \\
\hline \multirow[t]{5}{*}{$X_{t, t+3}-\bar{X}_{t, t+3}$} & $\mid$ Residual Cash Holding $_{t} \mid$ & -1.10 & -0.01 & -0.42 & 0.03 & -0.04 & -0.06 \\
\hline & & $(-5.54)^{* * *}$ & $(-0.18)$ & $(-16.56)^{* * *}$ & $(1.99)^{* *}$ & $(-1.27)$ & $(-3.40)^{* * *}$ \\
\hline & $\begin{array}{l}\mid \text { Residual Cash Holding }_{t} \mid \times \\
\text { Negative Dummy }\end{array}$ & -1.01 & 0.002 & -1.03 & $(-0.03)$ & -0.07 & -0.07 \\
\hline & & $(-4.99)^{* * *}$ & $(0.08)$ & $(-38.12)^{* * *}$ & $(-2.03)^{* *}$ & $(-2.20)^{* *}$ & $(-3.79)^{* * *}$ \\
\hline & $R^{2}$ & 0.05 & 0.01 & 0.33 & 0.02 & 0.03 & 0.02 \\
\hline
\end{tabular}


Figure 1. Time Series of Normal Cash and Residual Cash

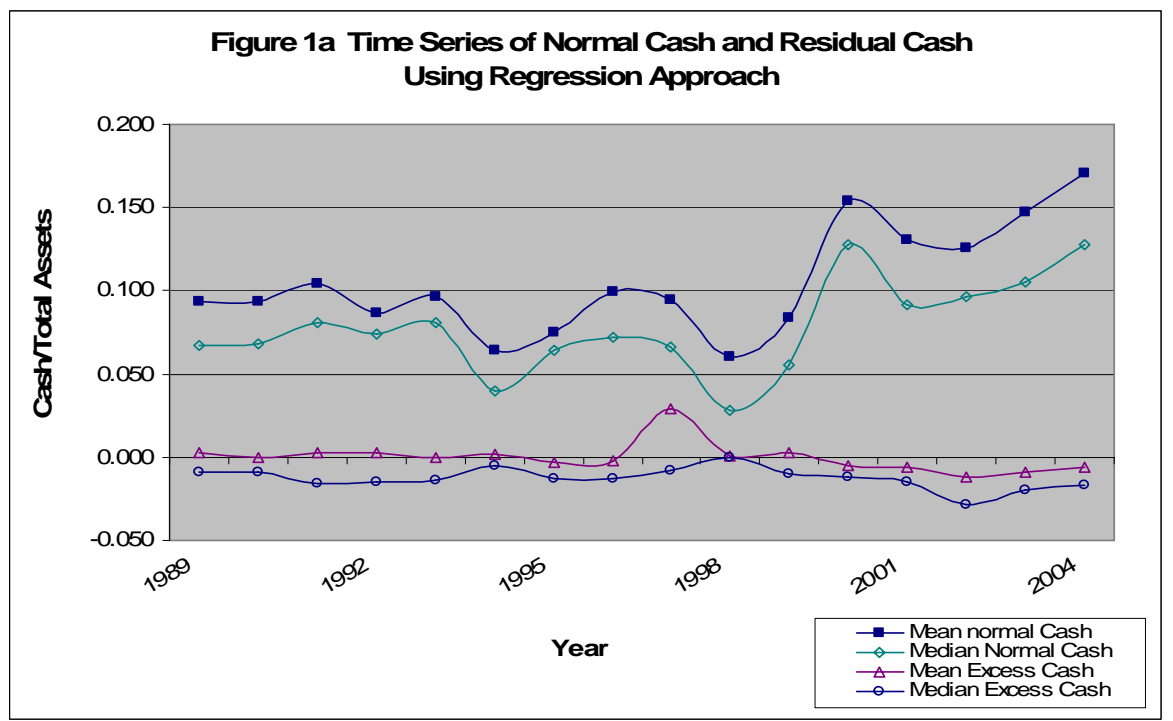

Figure 1a displays the time series of mean and median of normal cash holding and residual cash holding derived from using the following the regression model:

$$
\begin{aligned}
\text { Corporate Liqudity }_{i, t} & =\alpha+\beta_{1} \text { Assets }_{i, t}+\beta_{2} \frac{\text { CF }_{i, t}}{\text { Assets }_{i, t}}+\beta_{3} \frac{\text { NWC }_{i, t}}{\text { Assets }_{i, t}}+\beta_{4} \text { MKTtoBK }_{i, t}+\beta_{5} \frac{\text { CapitalExpense }_{i, t}}{\text { Assets }_{i, t}}+\beta_{6} \frac{R \& D_{i, t}}{\text { Sales }_{i, t}}+\beta_{7} \text { Leverage }_{i, t} \\
& +\beta_{8} \text { Dividend }_{i, t}+\beta_{9} \text { Bond Rating }_{i, t}+\beta_{10} G-\text { Index }_{i, t}+\text { Year Dummies }+ \text { Firm Fixed Effects }+\varepsilon_{i, t}
\end{aligned}
$$

Normal cash holding is the predicted value and the residual cash holding is the difference between the actual cash held by the firms and the "optimal" or "normal" cash holdings predicted by the above regression equation.

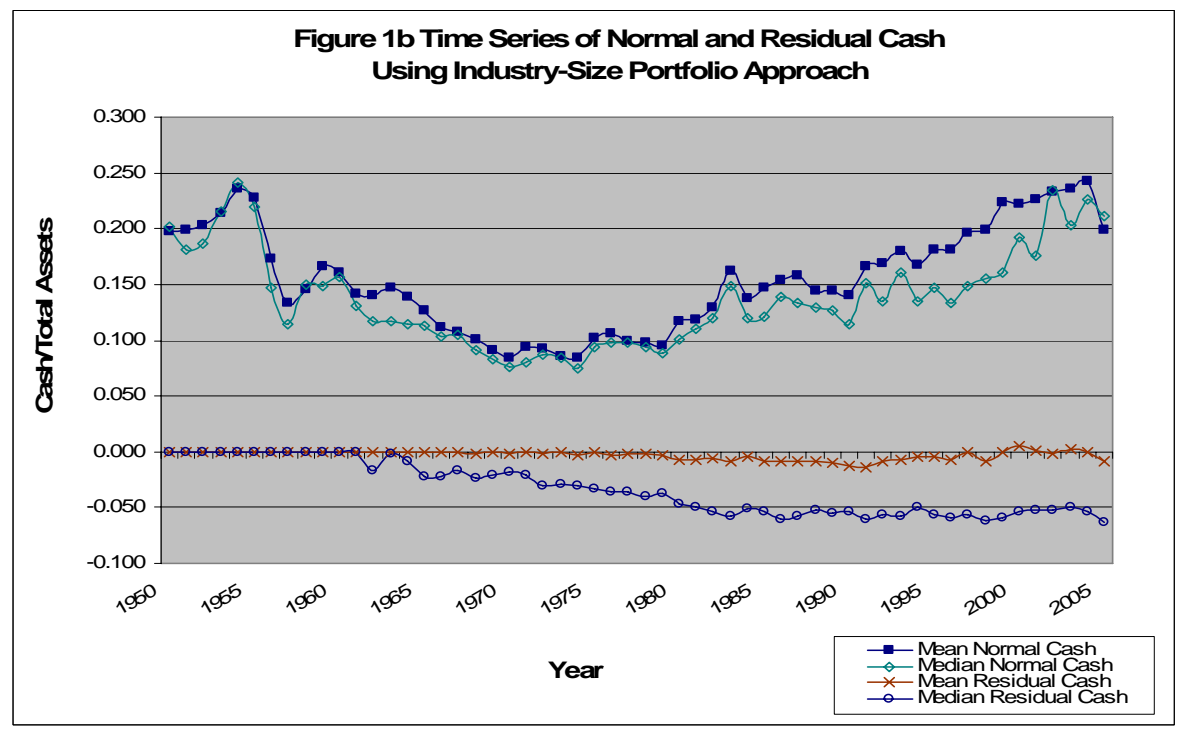

Figure $1 \mathrm{~b}$ shows the mean and median of normal and residual cash holding estimated using industry-size portfolio average approach. First, each period, all the firms are categorized into 10 industry portfolios defined by Fama and French; then within each industry portfolio, firms are subsequently sorted into 5 size sub portfolios based on firms' market equity. Next, the industry-size corporate liquidity norm is calculated each period by taking the average of cash holdings of all firms falling into a certain industry-size portfolio. The normal cash holding of an individual firm is the corporate liquidity of the industry-size portfolio which the firm belongs to during a specific period. The residual cash holding is the difference between actual corporate liquidity and the normal cash holding for a specific period. 
Figure 2. Bankruptcies and Failures by Year

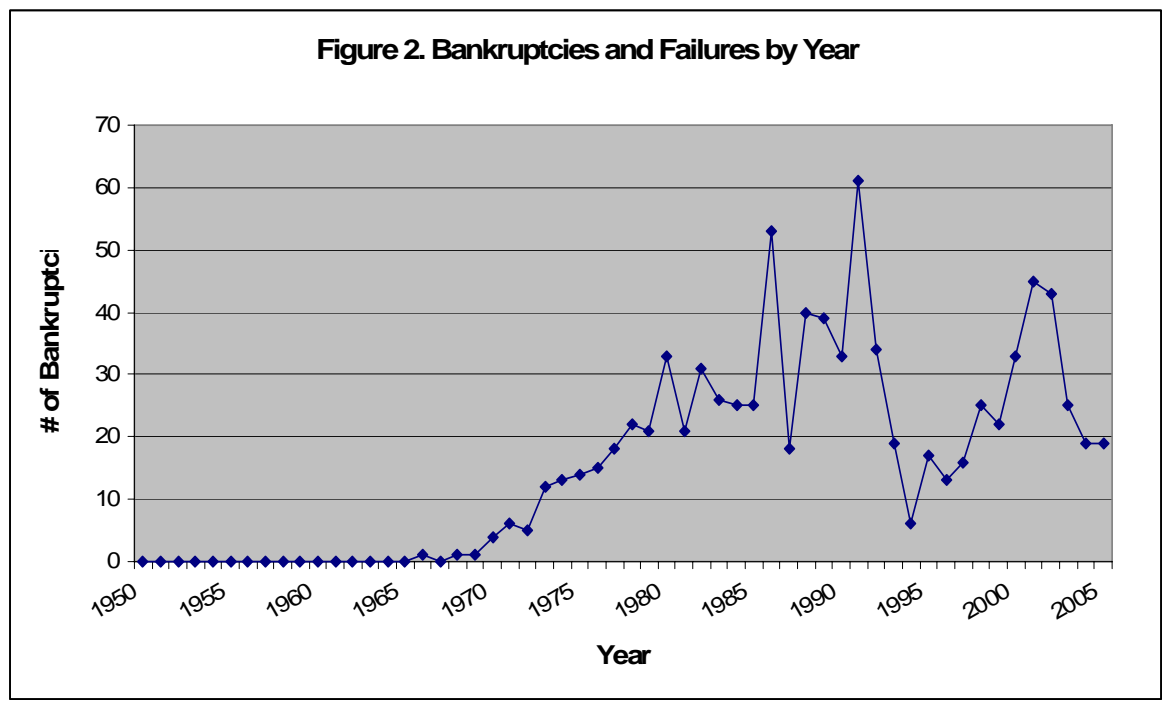

Figure 2 shows the number of bankruptcies per year over the sample period of $1950-2005$. The bankruptcy sample includes all bankruptcy filings as reported in the COMPUSTAT and CRSP datasets. Firms with a CRSP bankruptcy delisting code 400-490 (liquidations), 572 (liquidations) and 574 (bankruptcy), and firms with COMPUSTAT footnote 35 having code 02 (Chapter 11) or 03 (Chapter 7) are considered as failing and will be included in the bankruptcy sub sample. There are a total of 874 bankruptcies in the sample.

Figure 3. Bankruptcies and Failures as Percentage of Active Firms

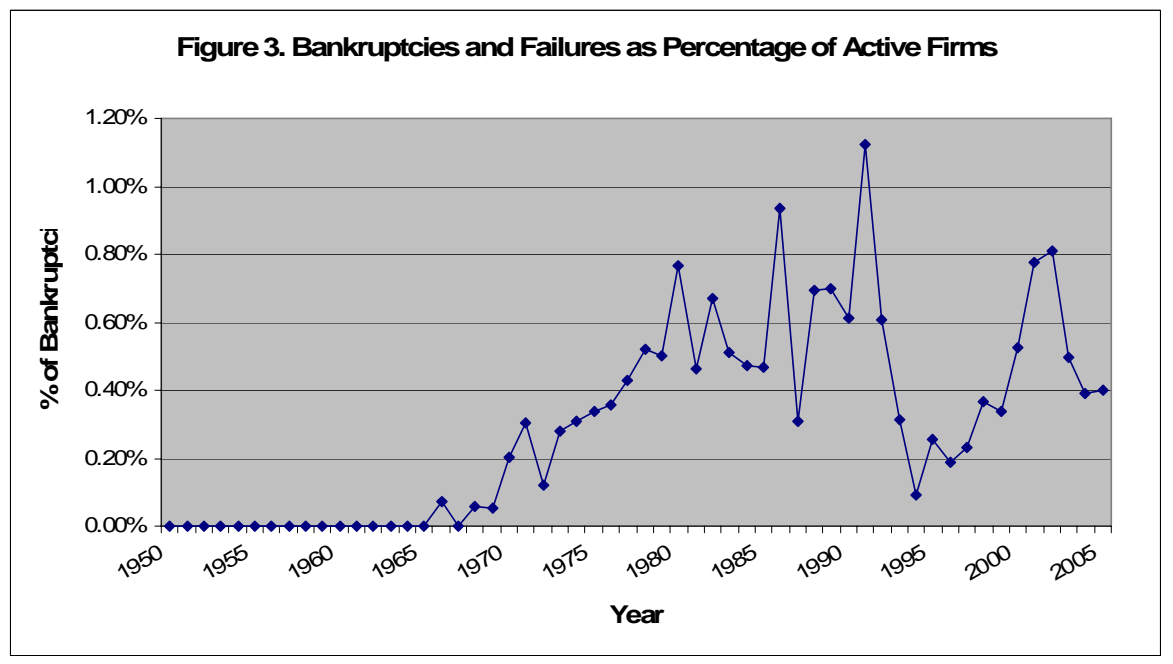

Figure 3 shows bankruptcies as percentage of all the active firms per year over the sample period of 1950 -2005. The bankruptcy sample includes all bankruptcy filings as reported in the COMPUSTAT and CRSP datasets. Firms with a CRSP bankruptcy delisting code 400-490 (liquidations), 572 (liquidations) and 574 (bankruptcy), and firms with COMPUSTAT footnote 35 having code 02 (Chapter 11) or 03 (Chapter 7) are considered as failing and will be included in the bankruptcy sub sample. There are a total of 874 bankruptcies in the sample. 